
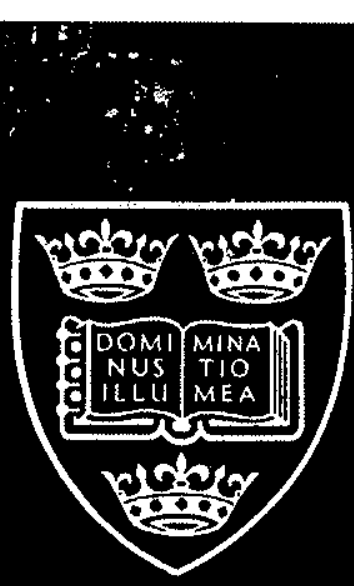

\title{
OXFORD UNIVERSITY
}

AN INTRODUCTION TO THE POSSIBLE SUBSTRUCTURE OF

QUARKS AND LEPTONS

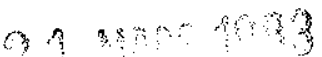

Louis Lyons

Updated Dec. 1982

\section{NUCLEAR PHYSICS LABORATORY}




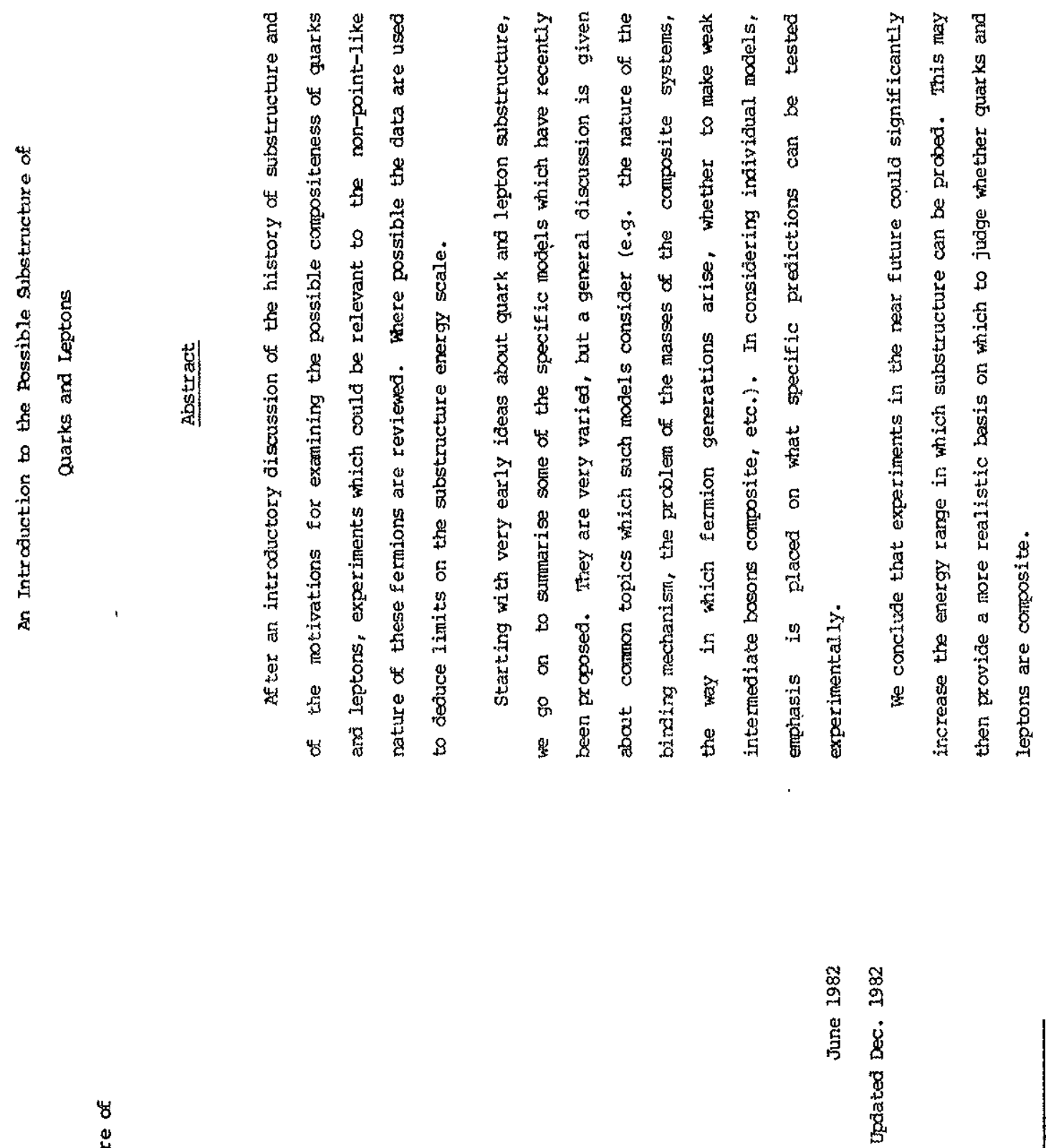

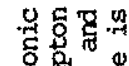

筞

"res

की

尊员

政

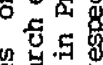

造

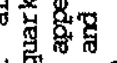

岁

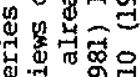

㟧

4.58 ?

of

s.

-

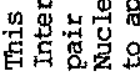



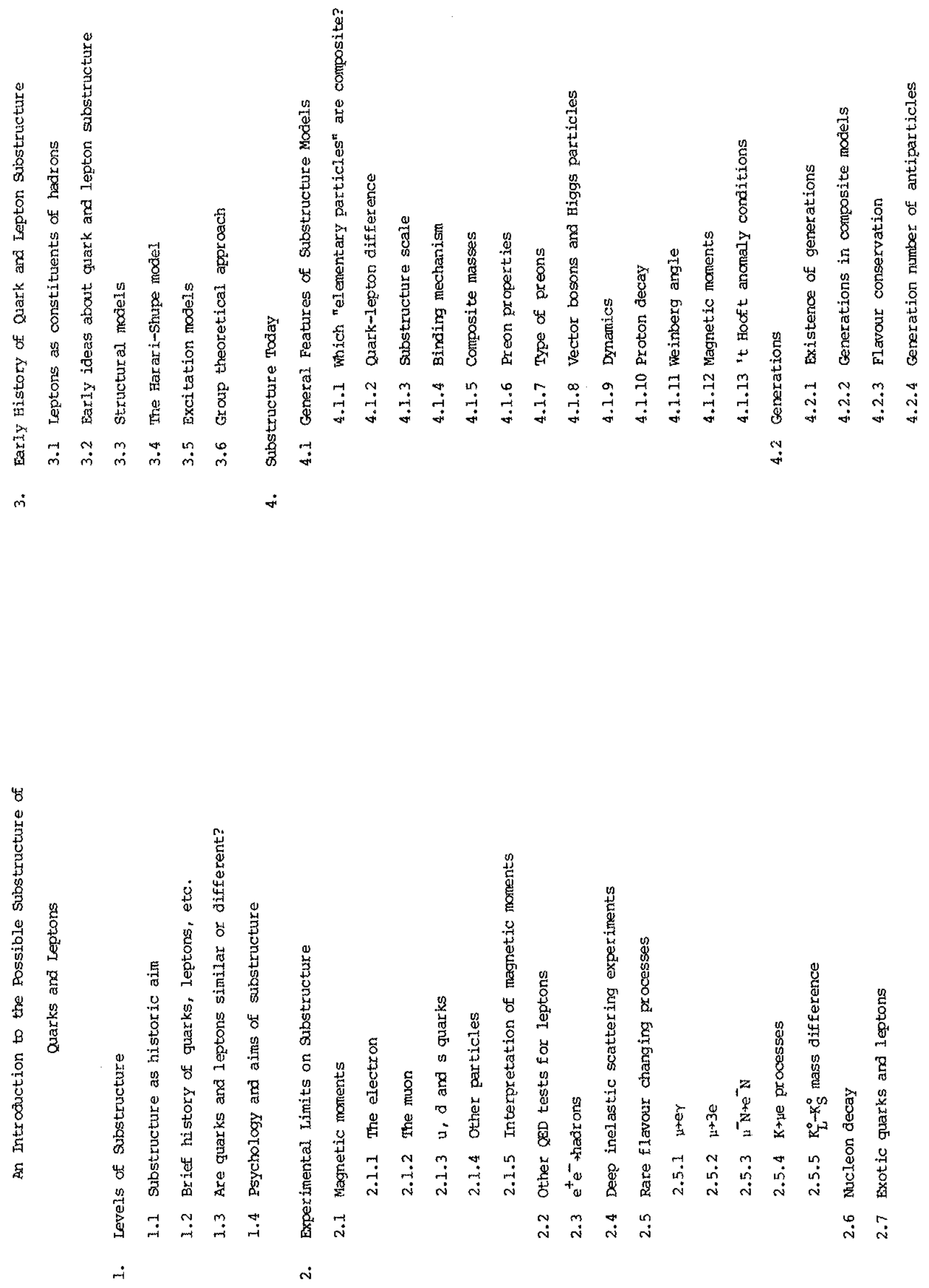


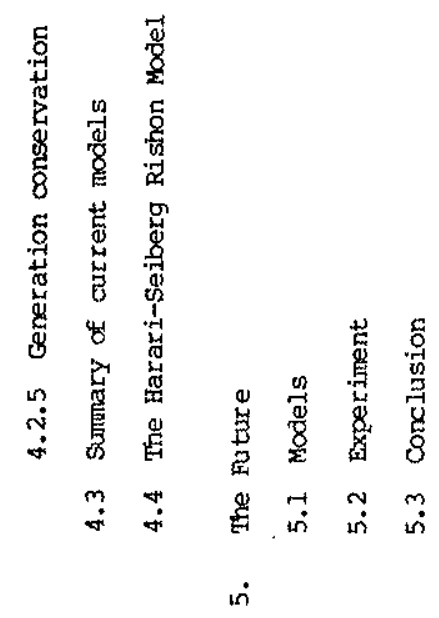




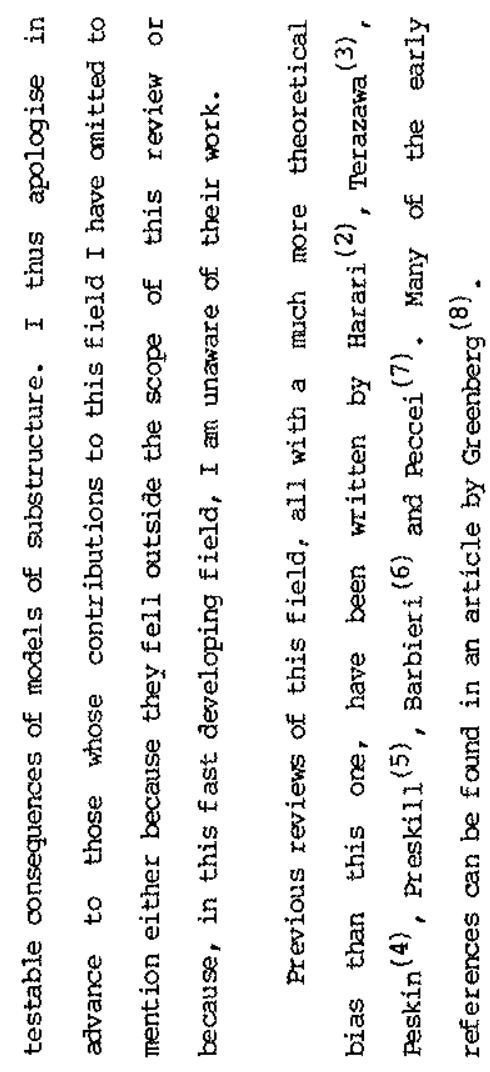

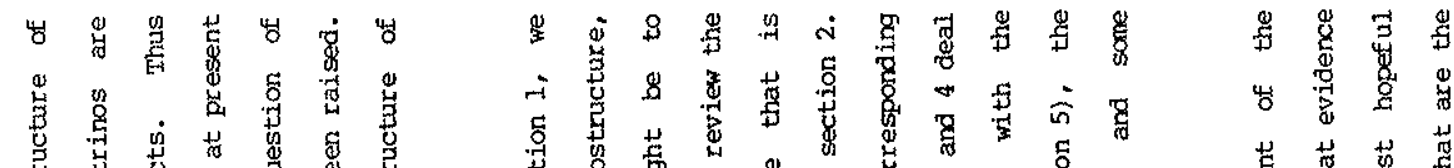

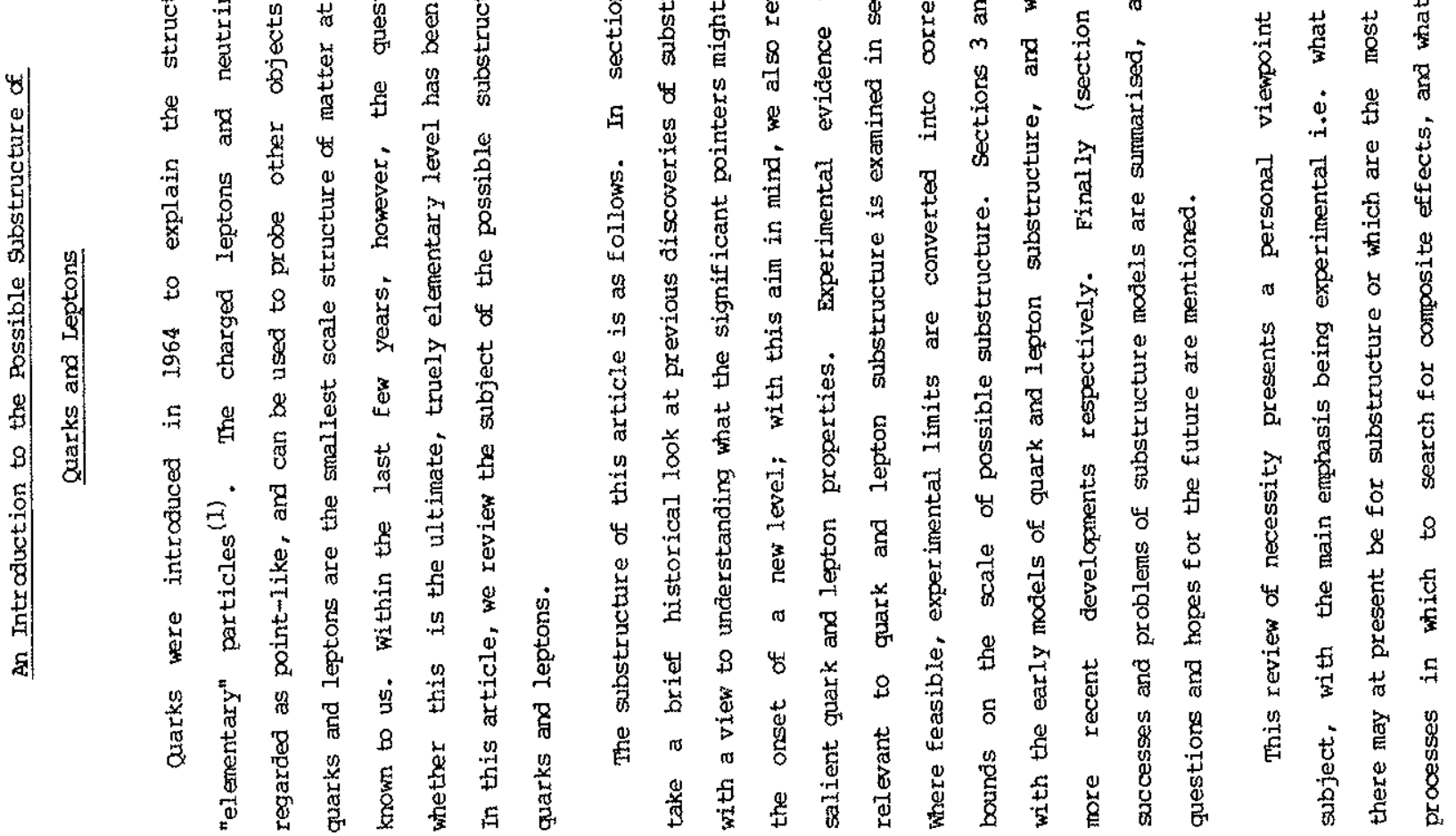




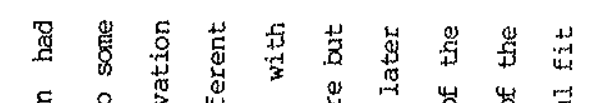

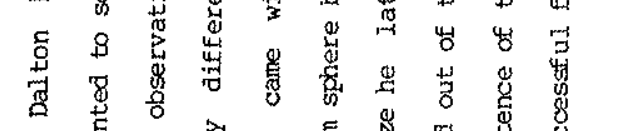

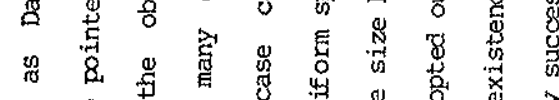

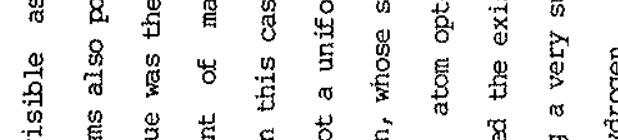

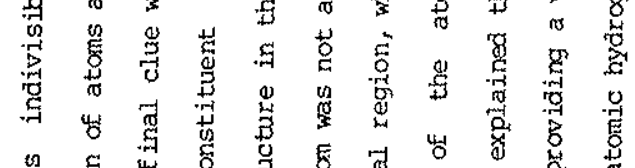

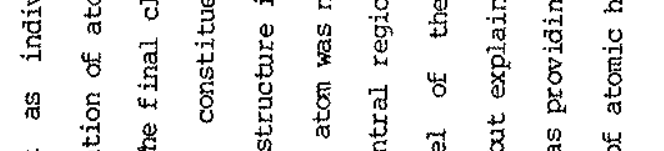

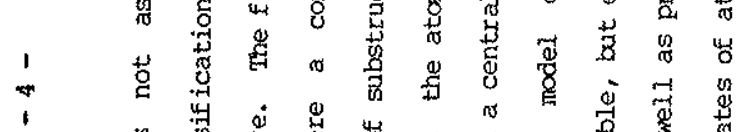

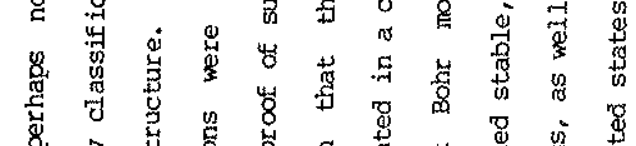

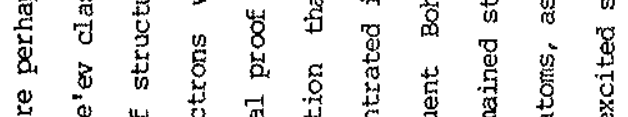

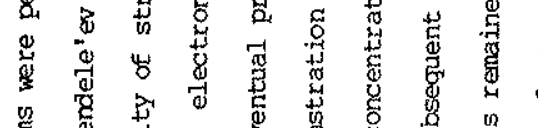

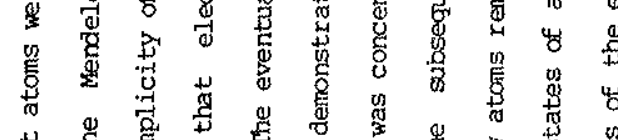

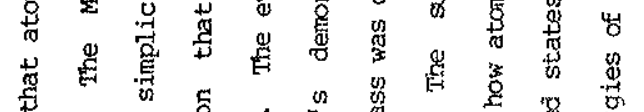

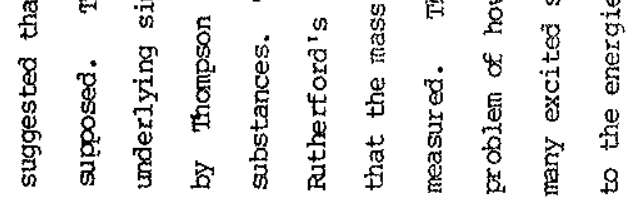

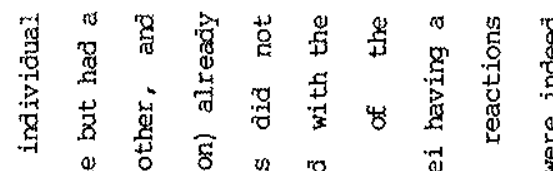

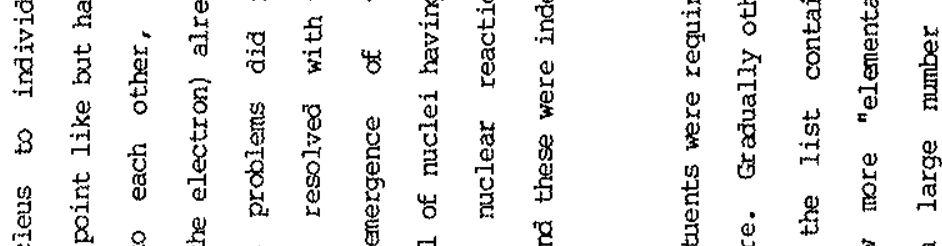

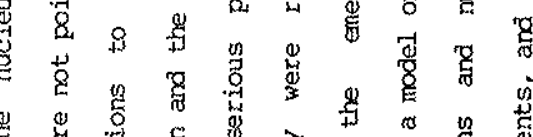

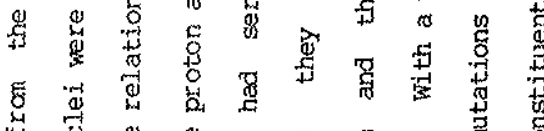

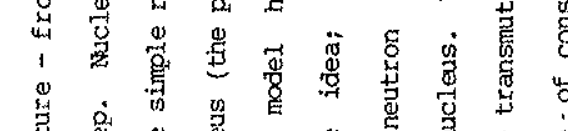

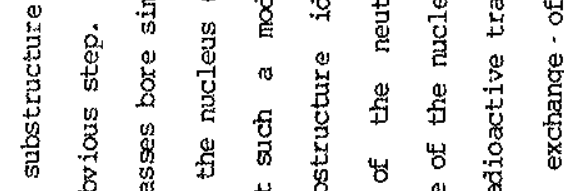

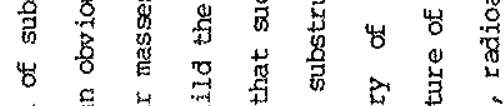

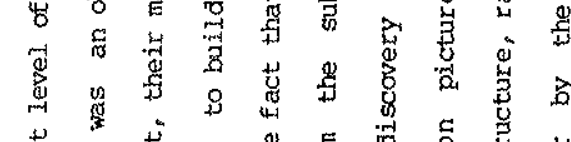

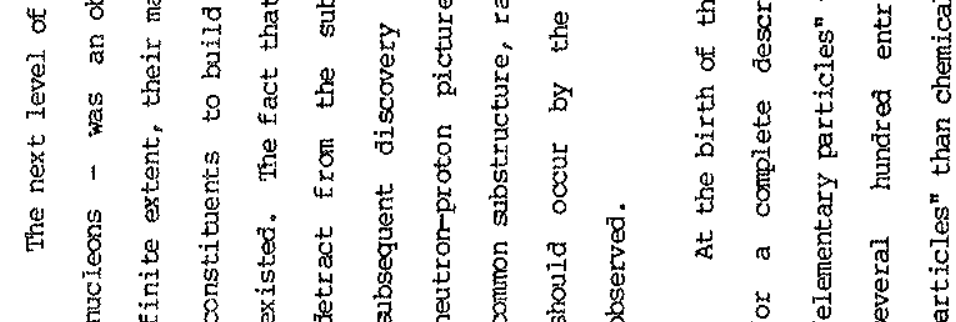

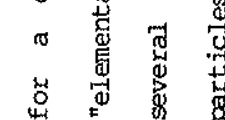

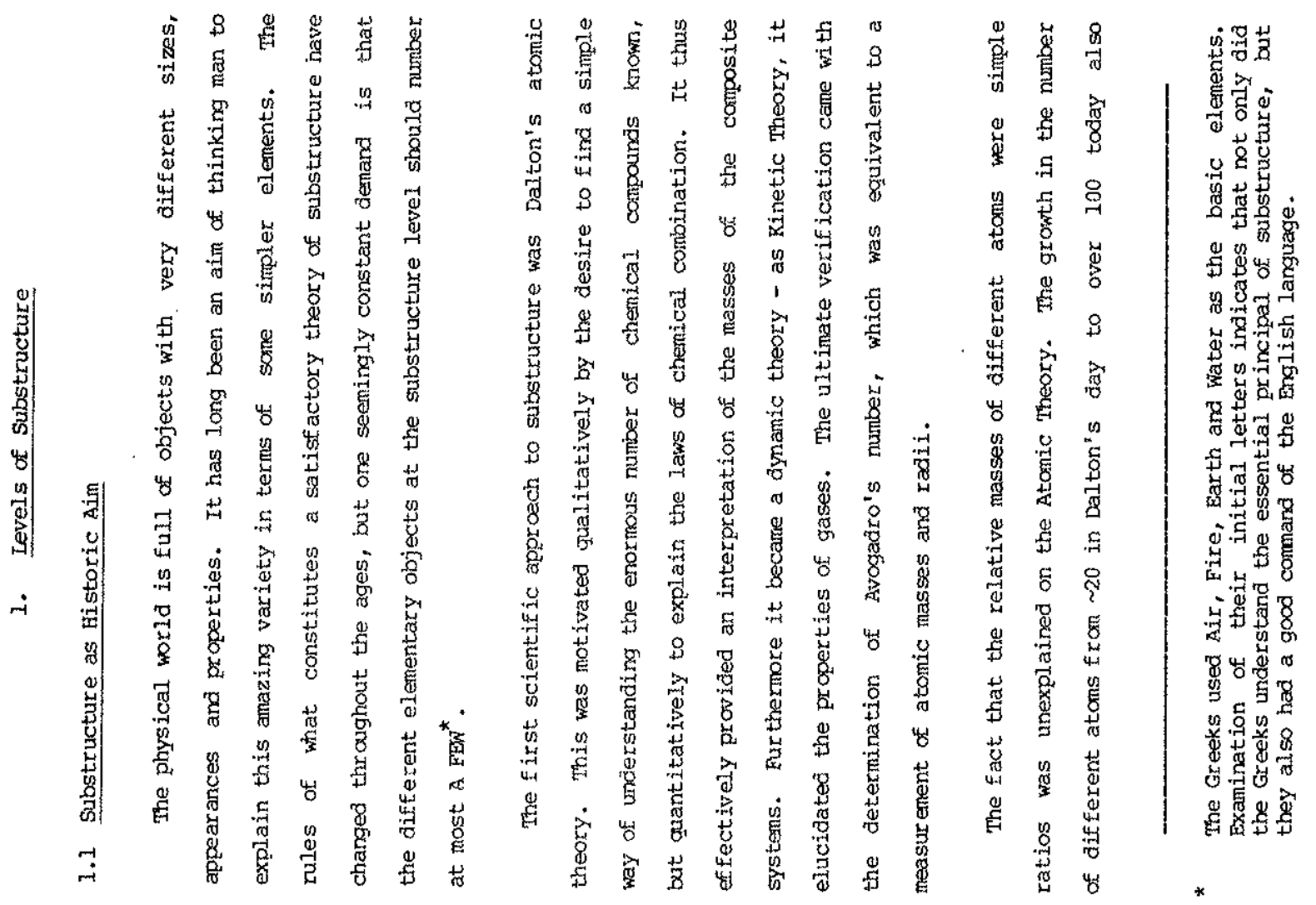




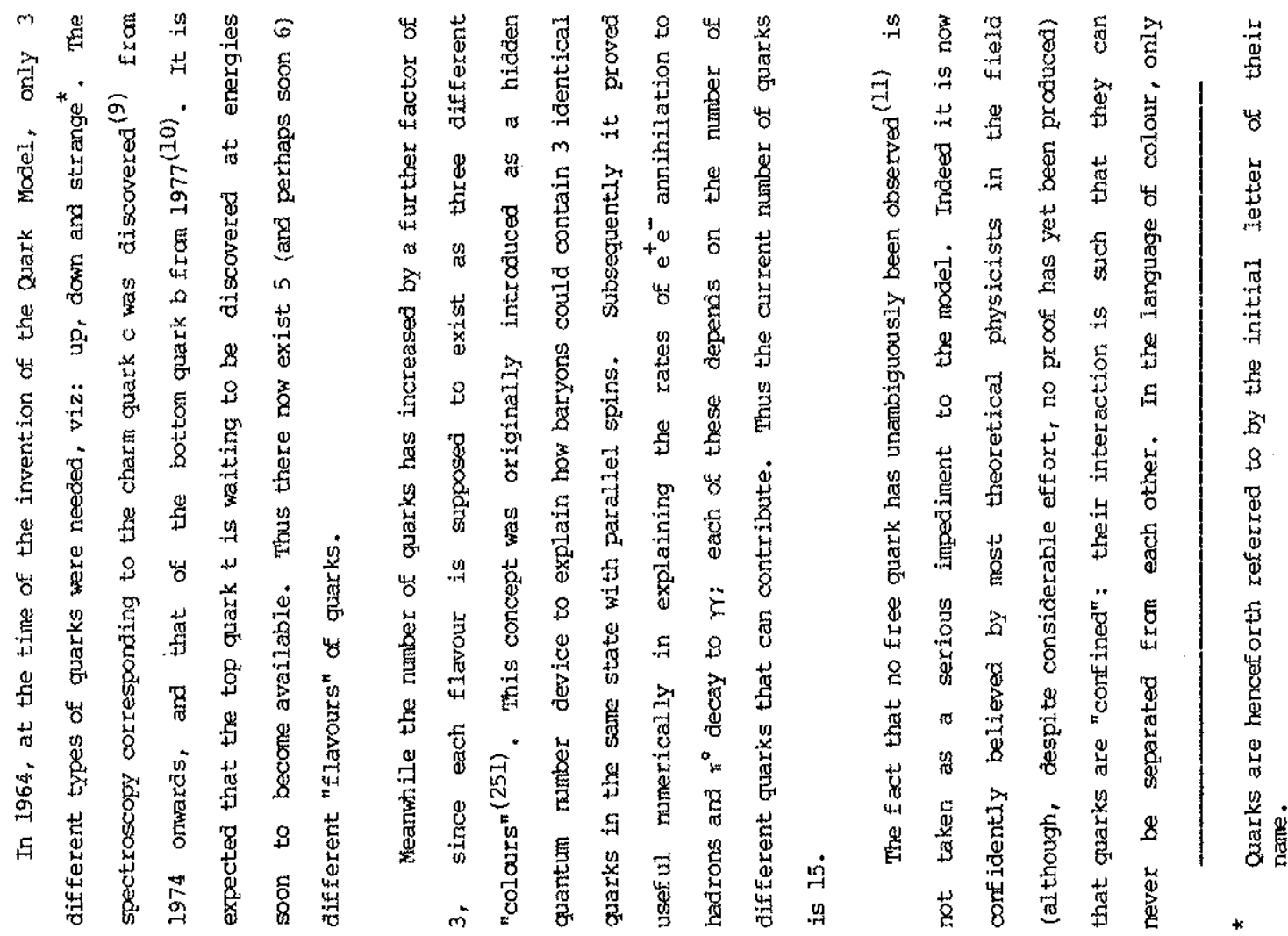

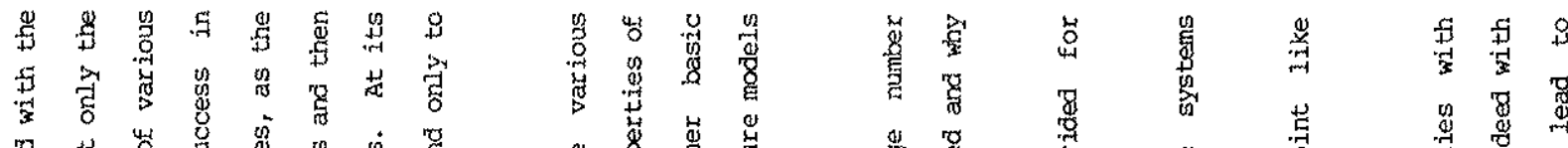

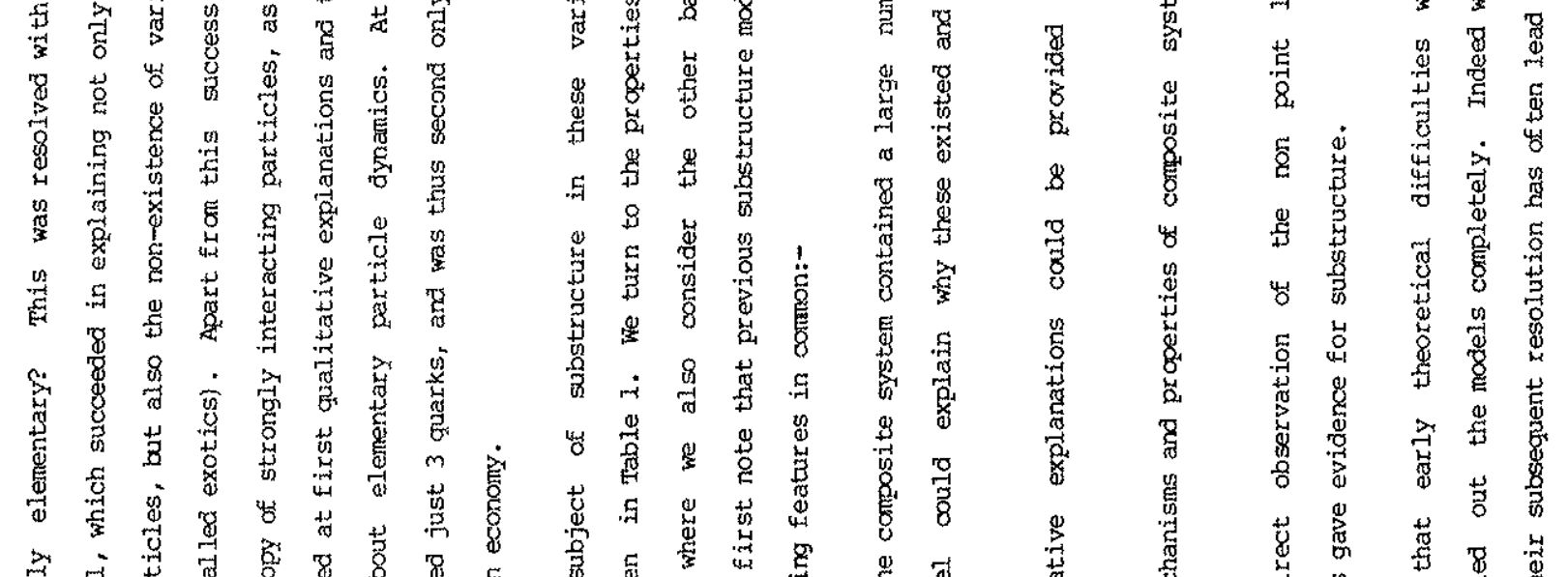

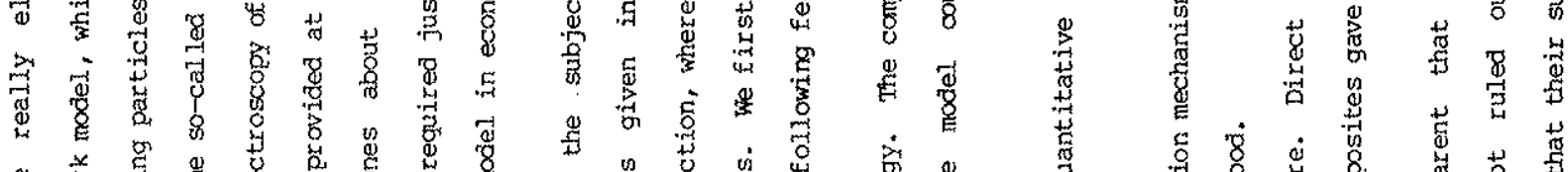

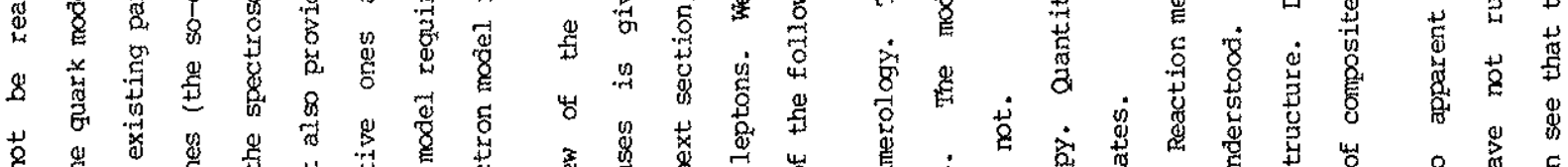

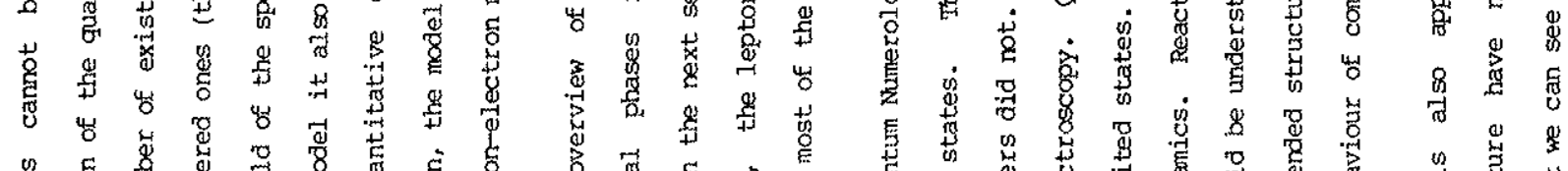

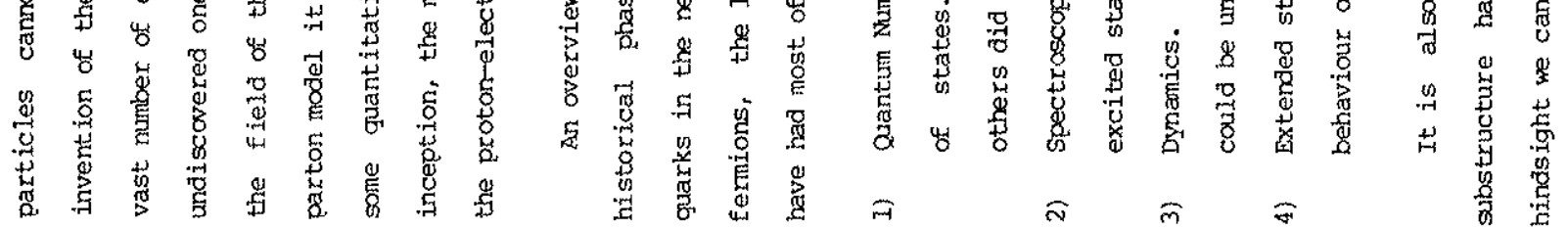



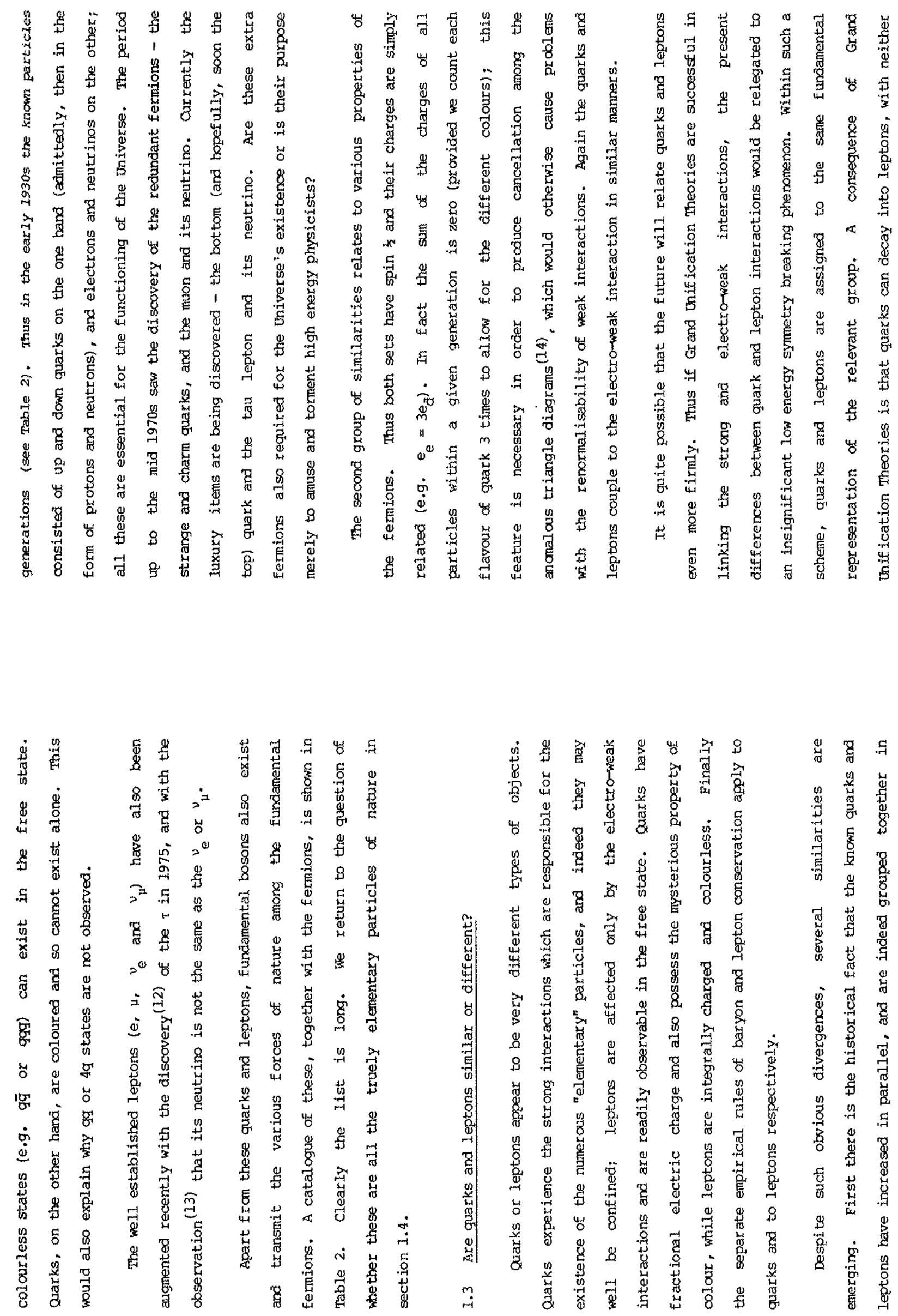

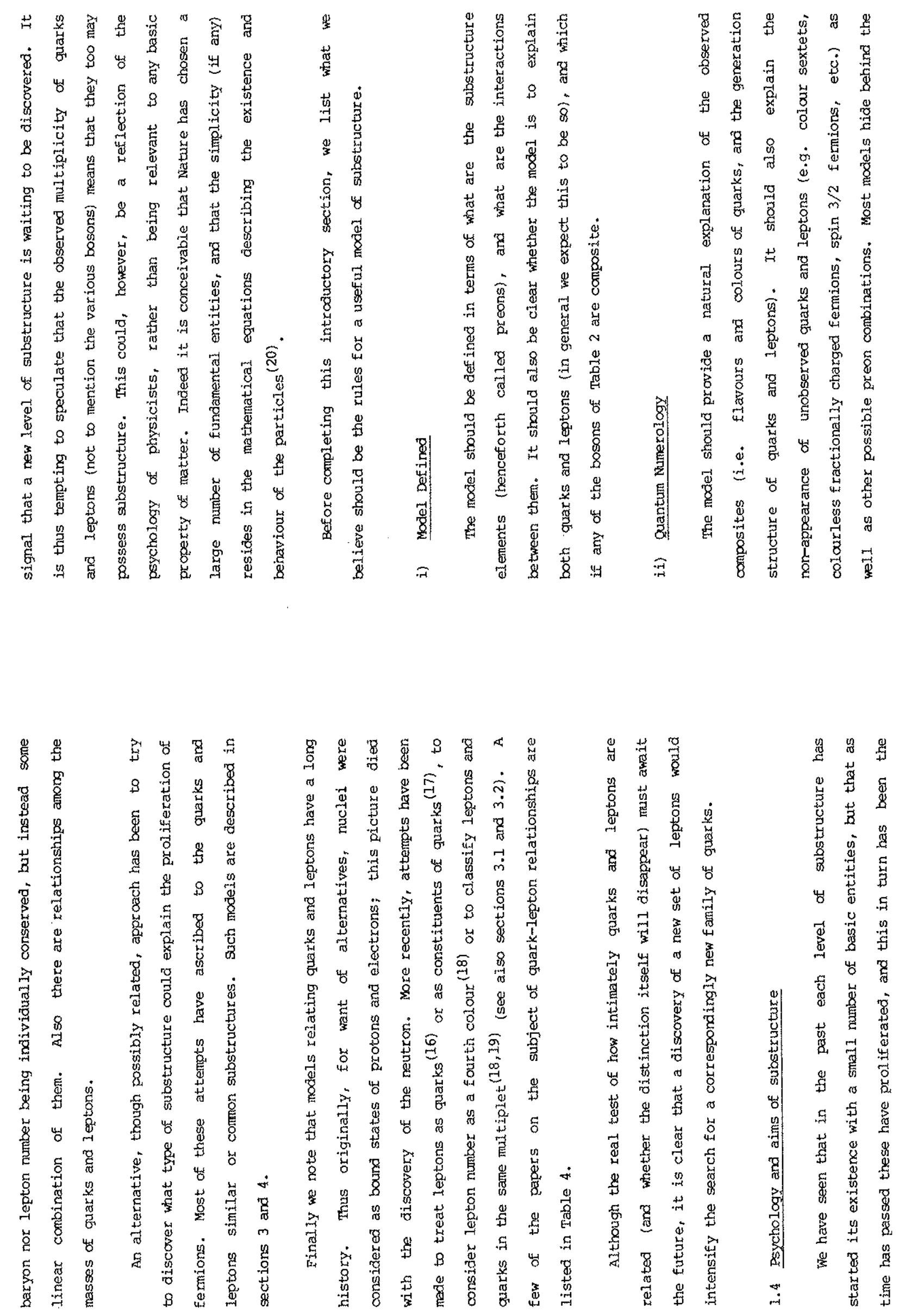

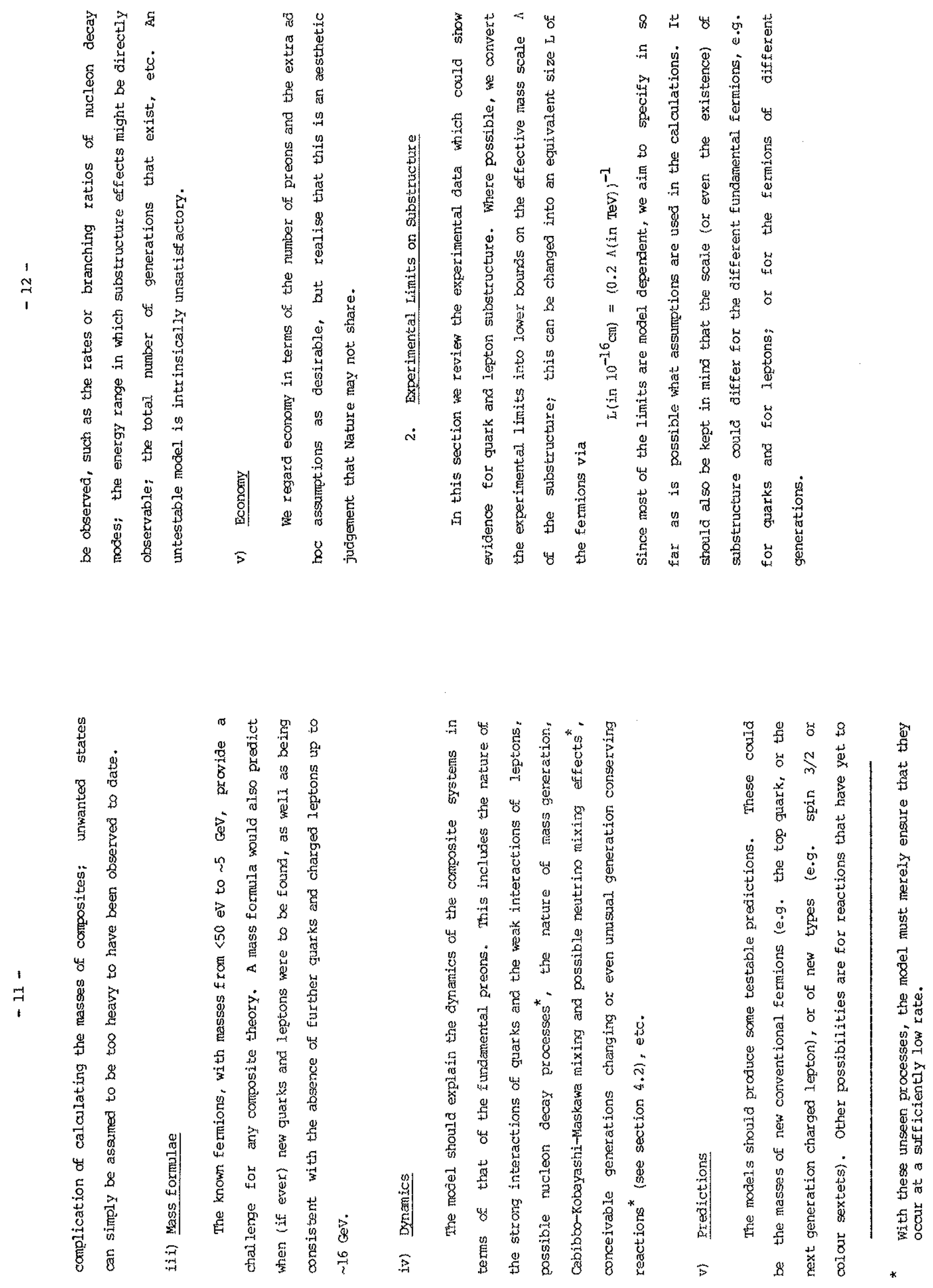

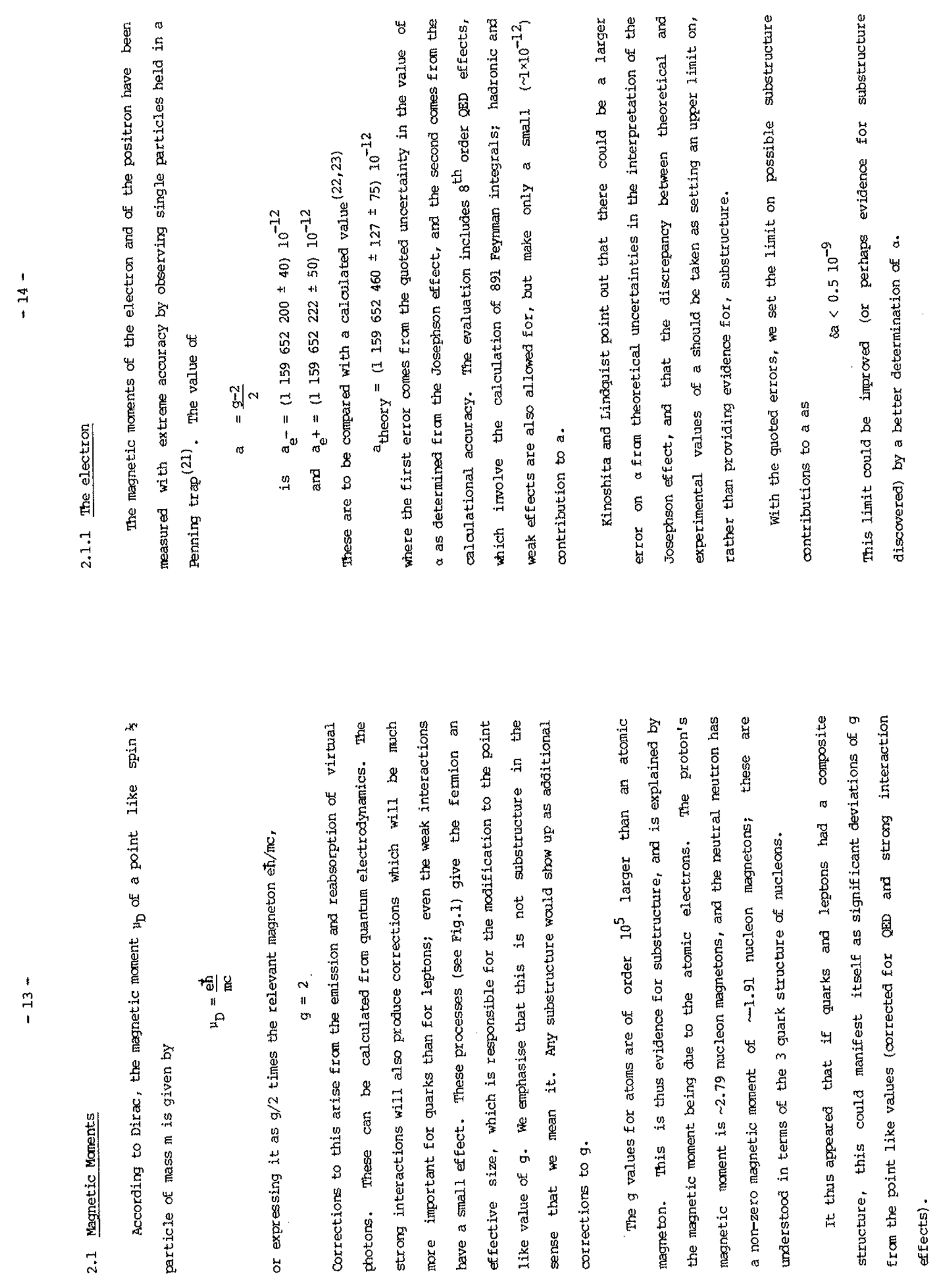

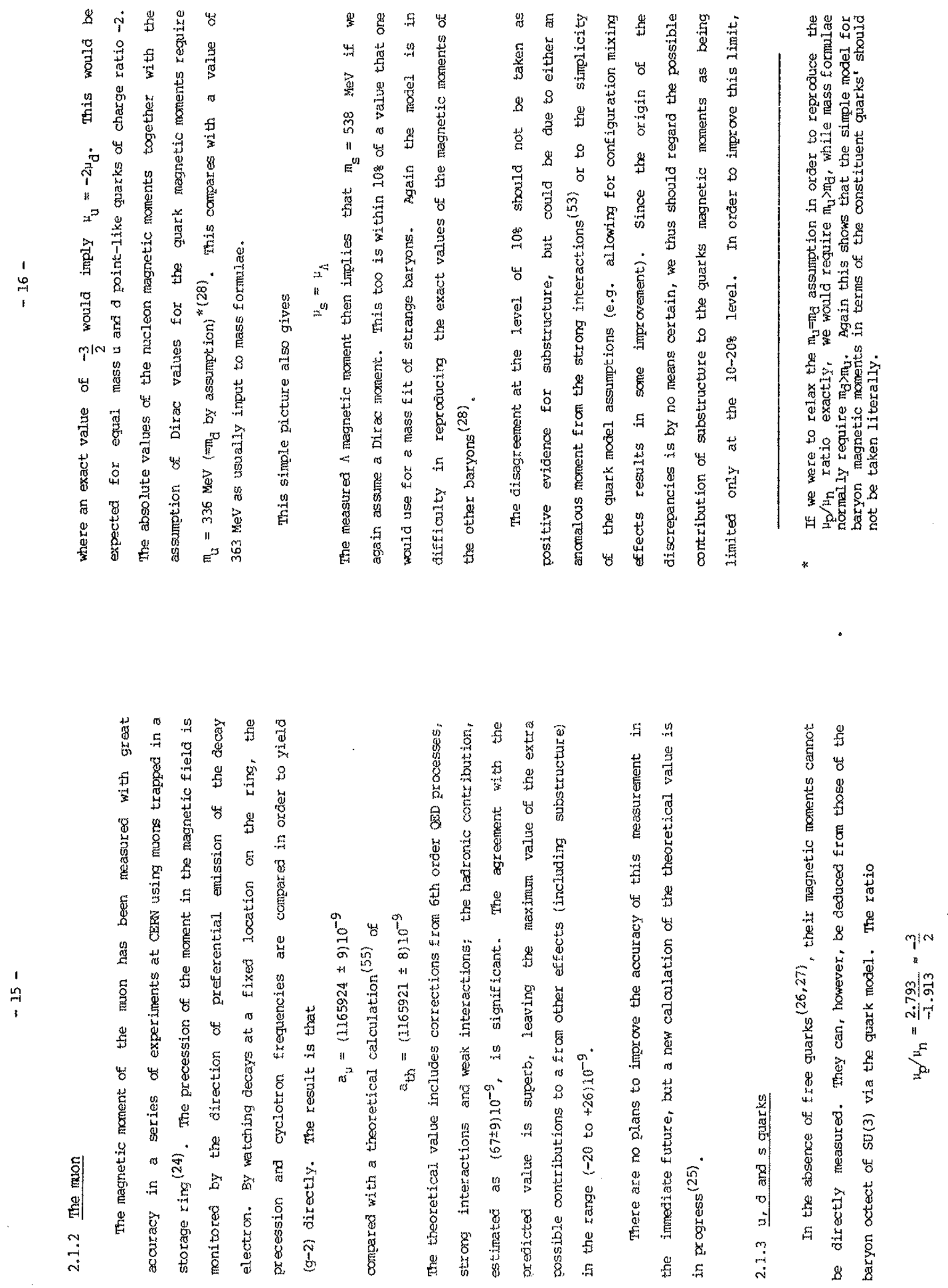

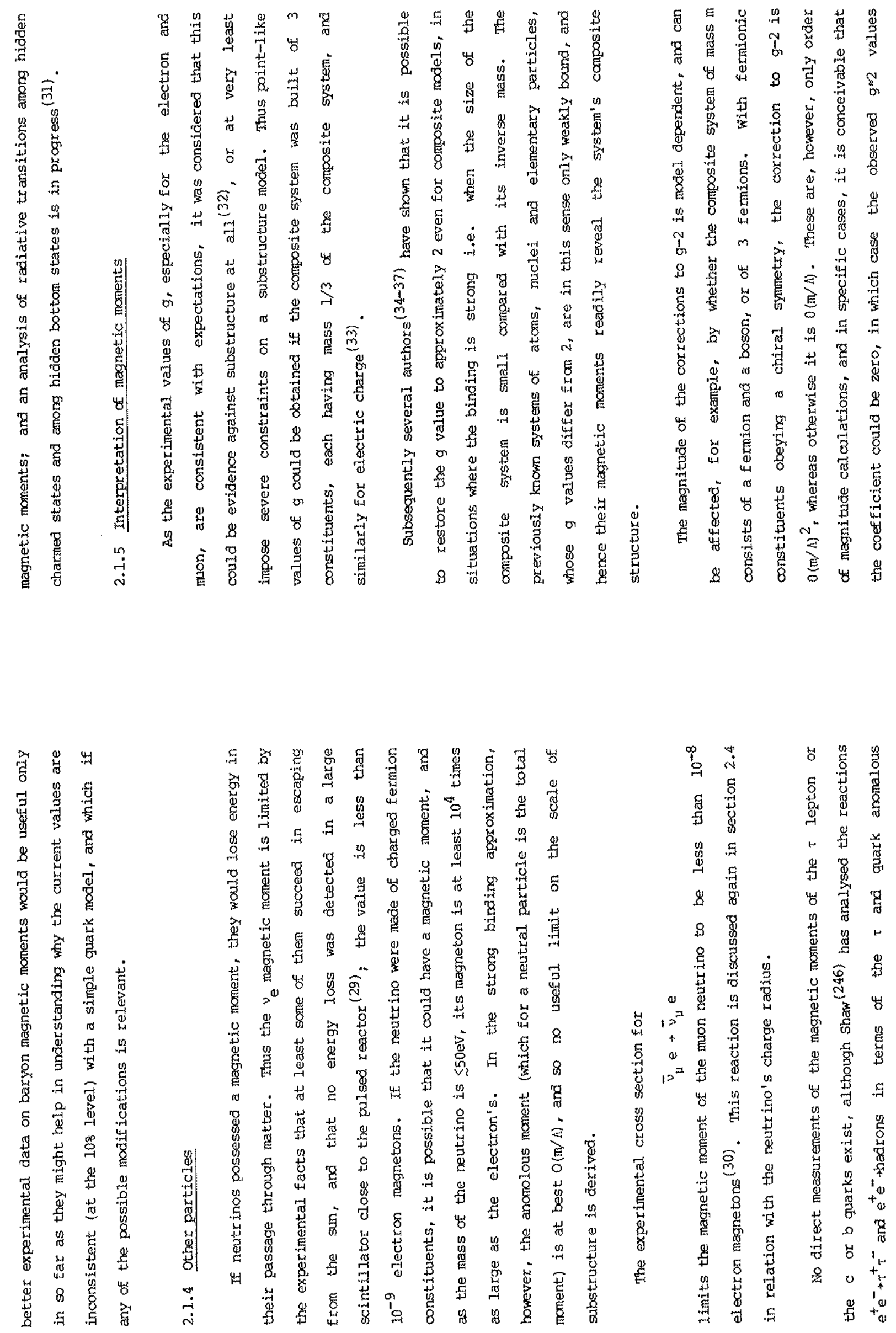


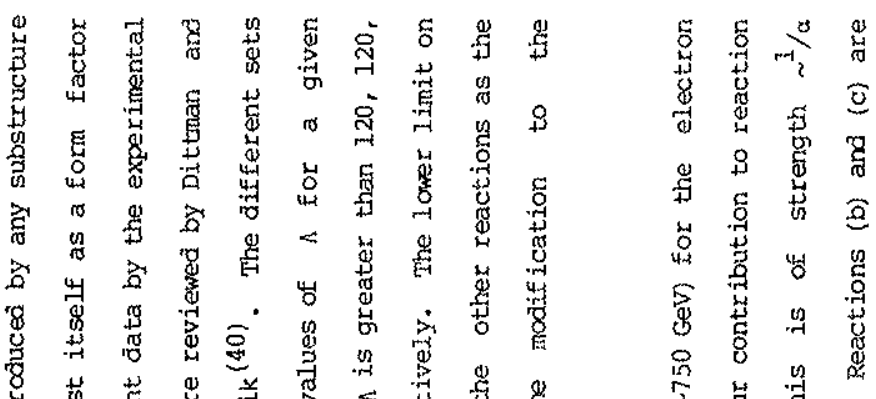

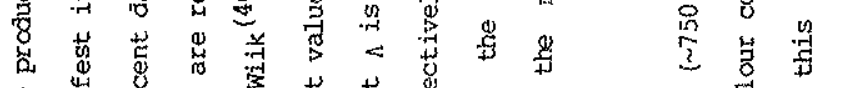

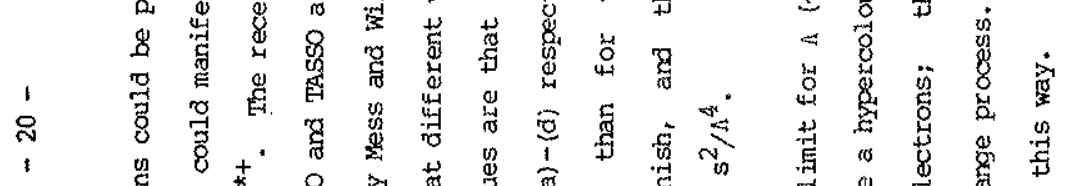

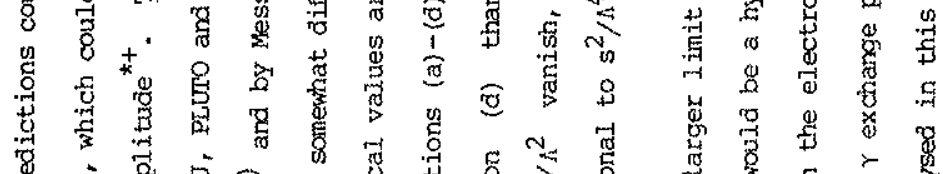

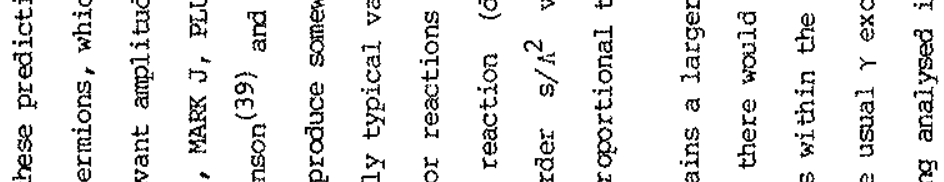

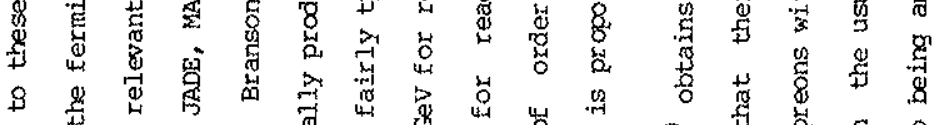

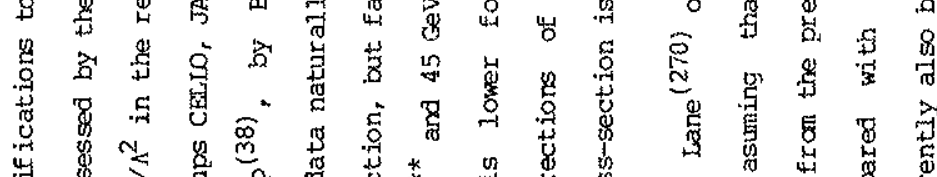

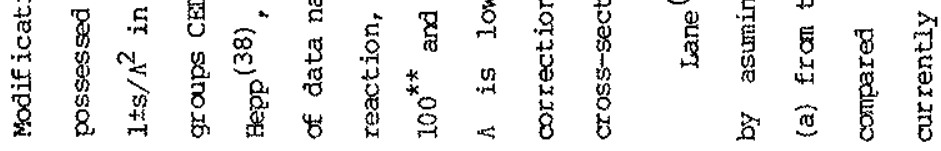

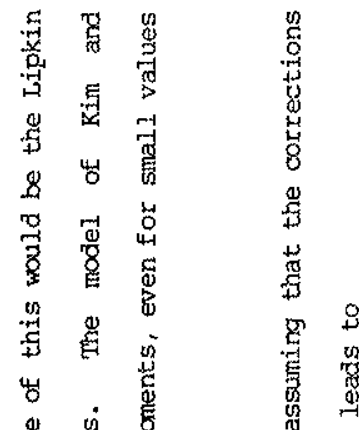

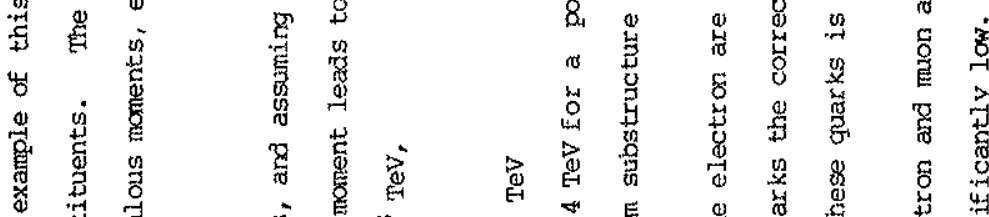

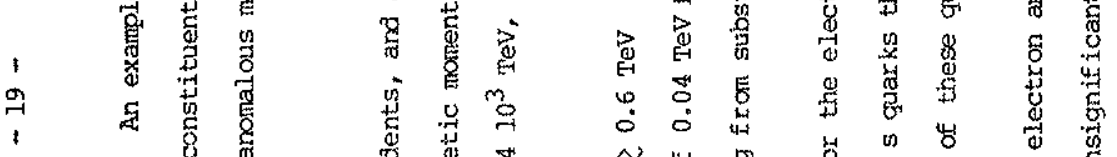

$\begin{array}{rlllll} & & \\ 0\end{array}$

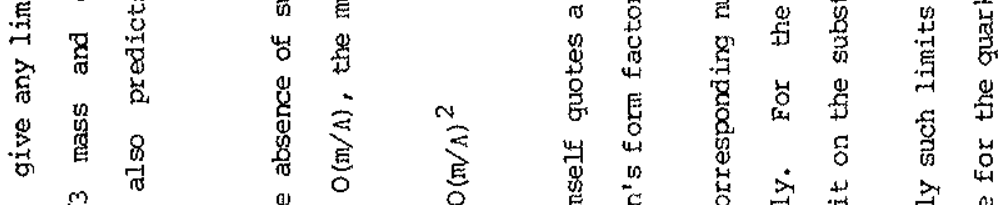

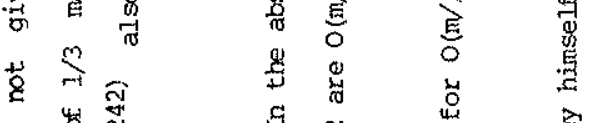

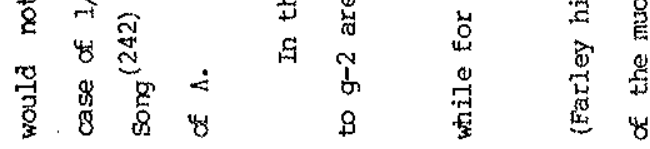

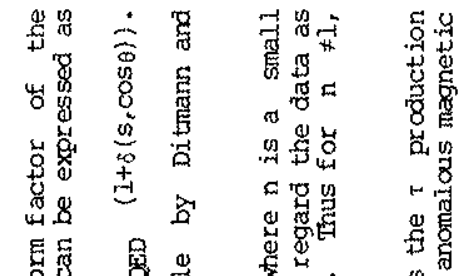

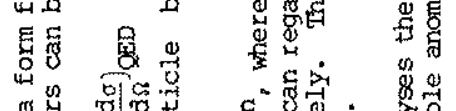

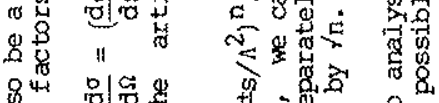

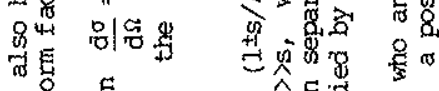

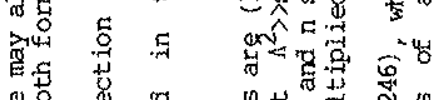

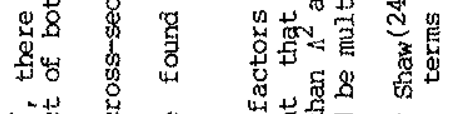

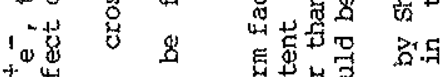

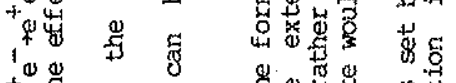

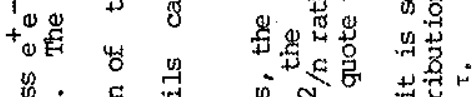

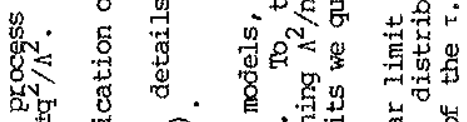

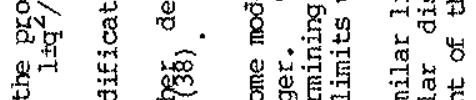

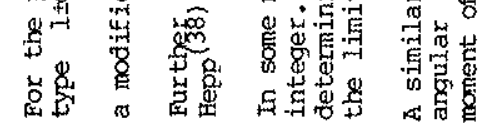

즐

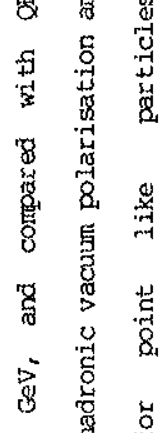

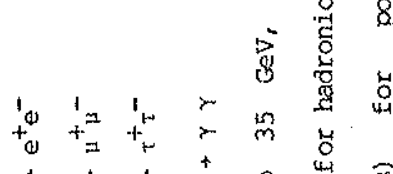

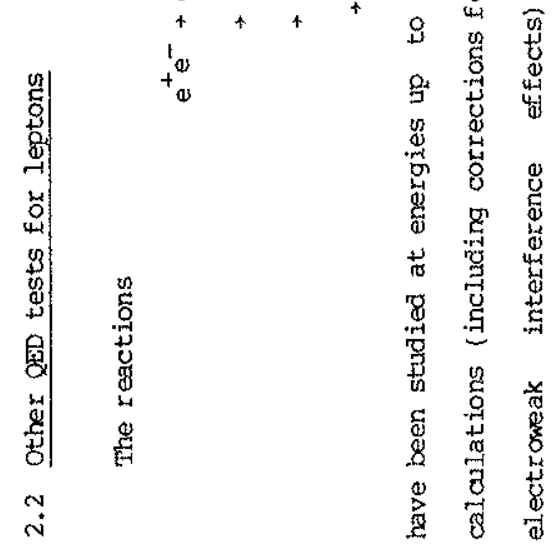



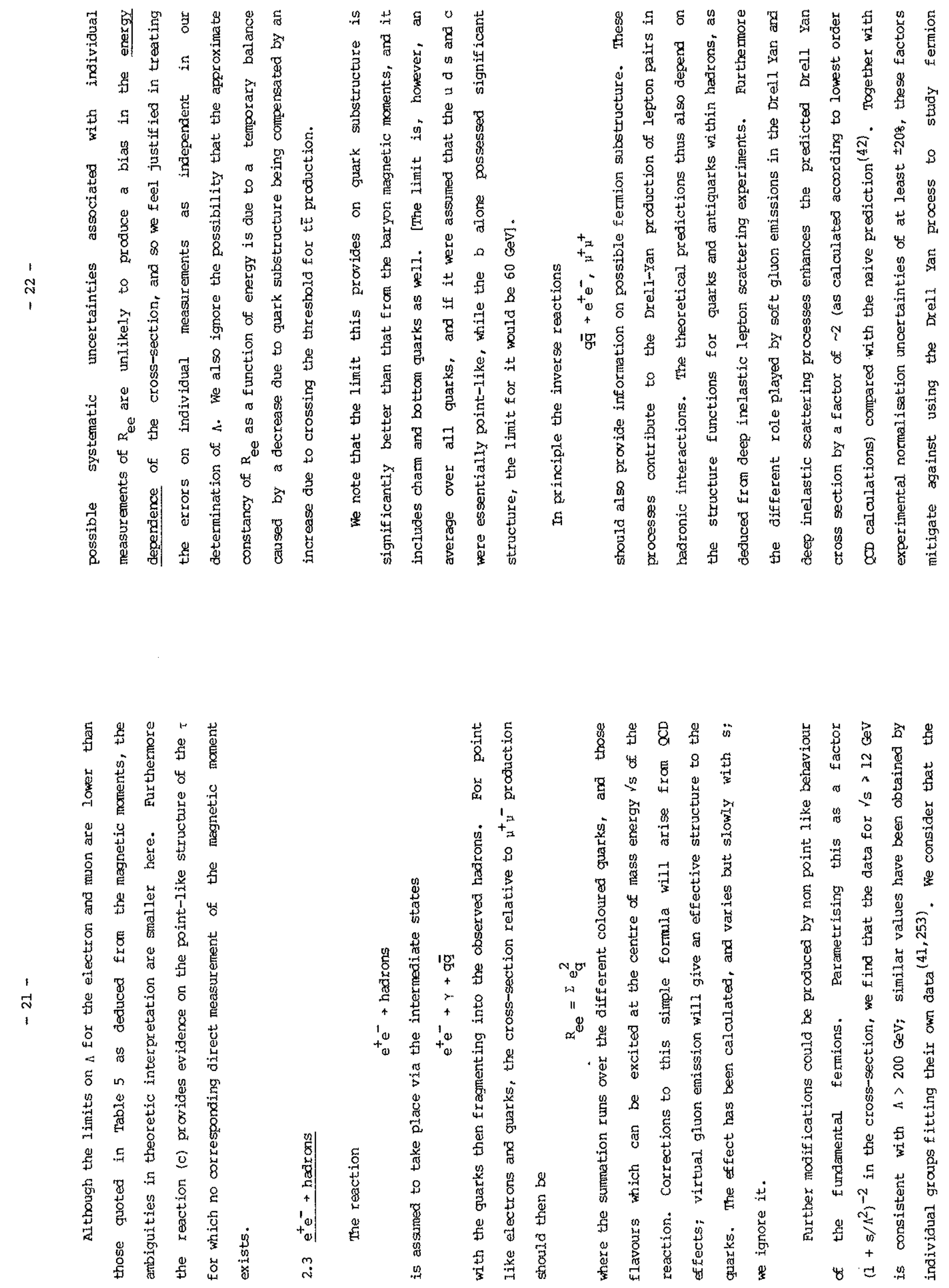

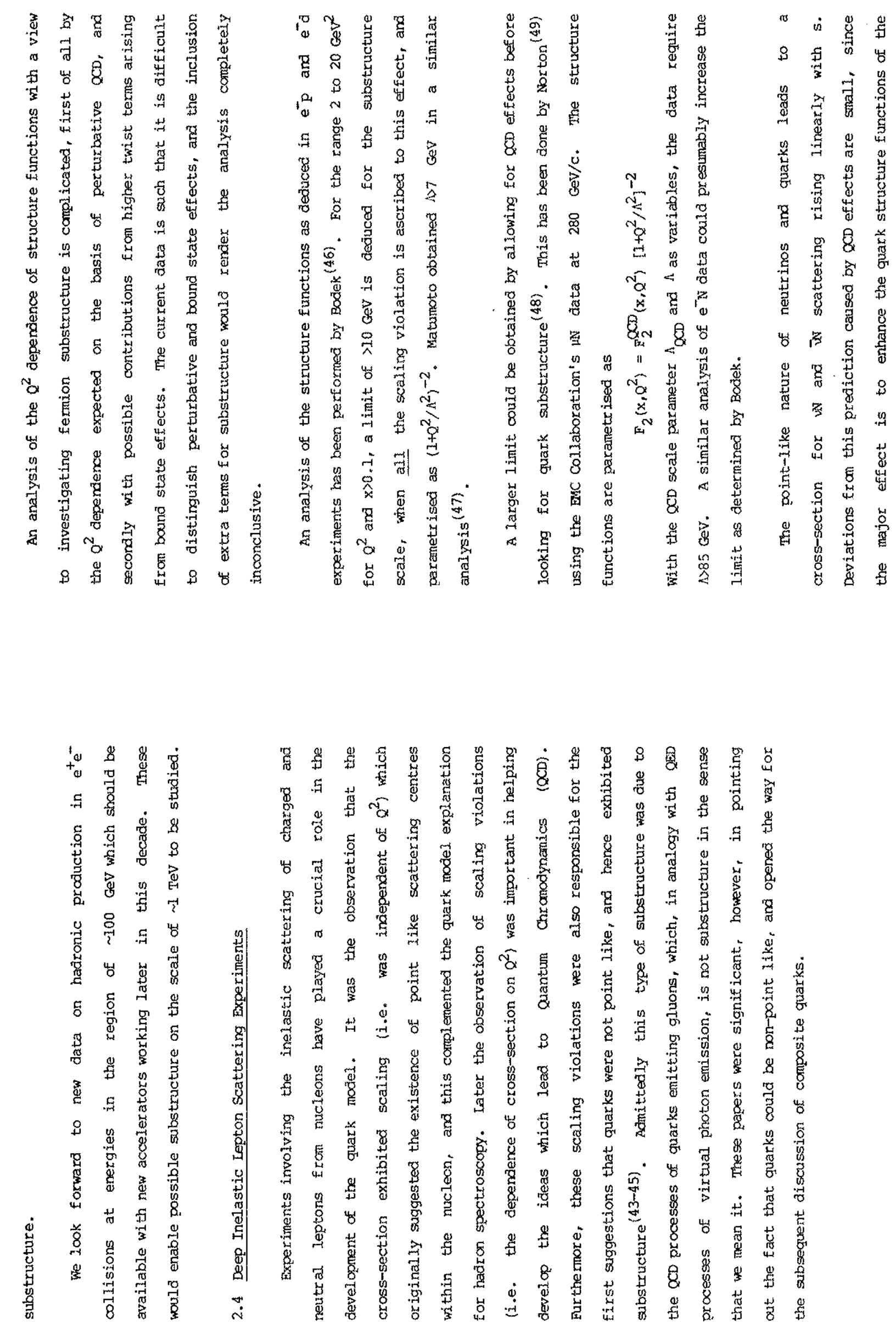

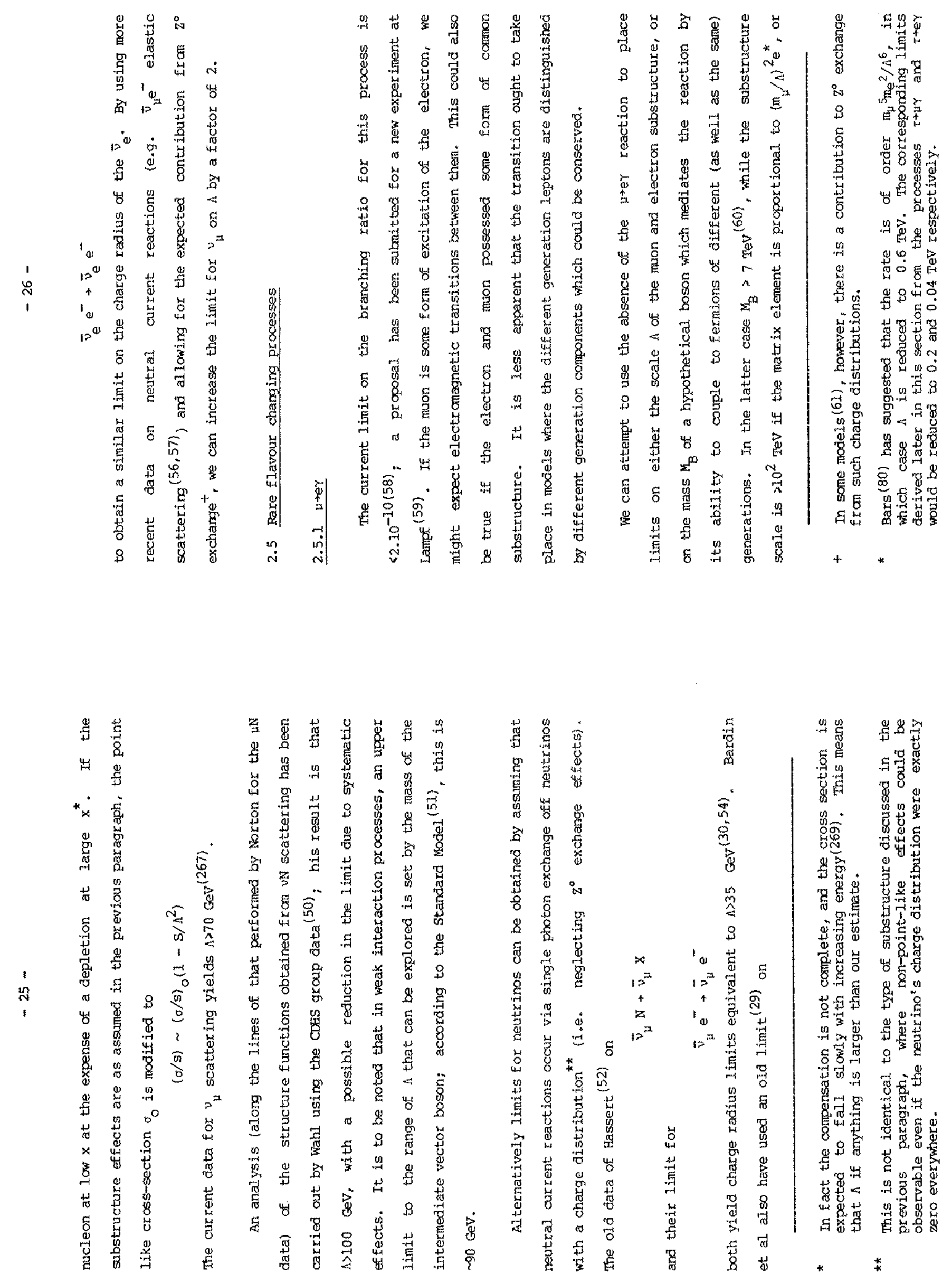


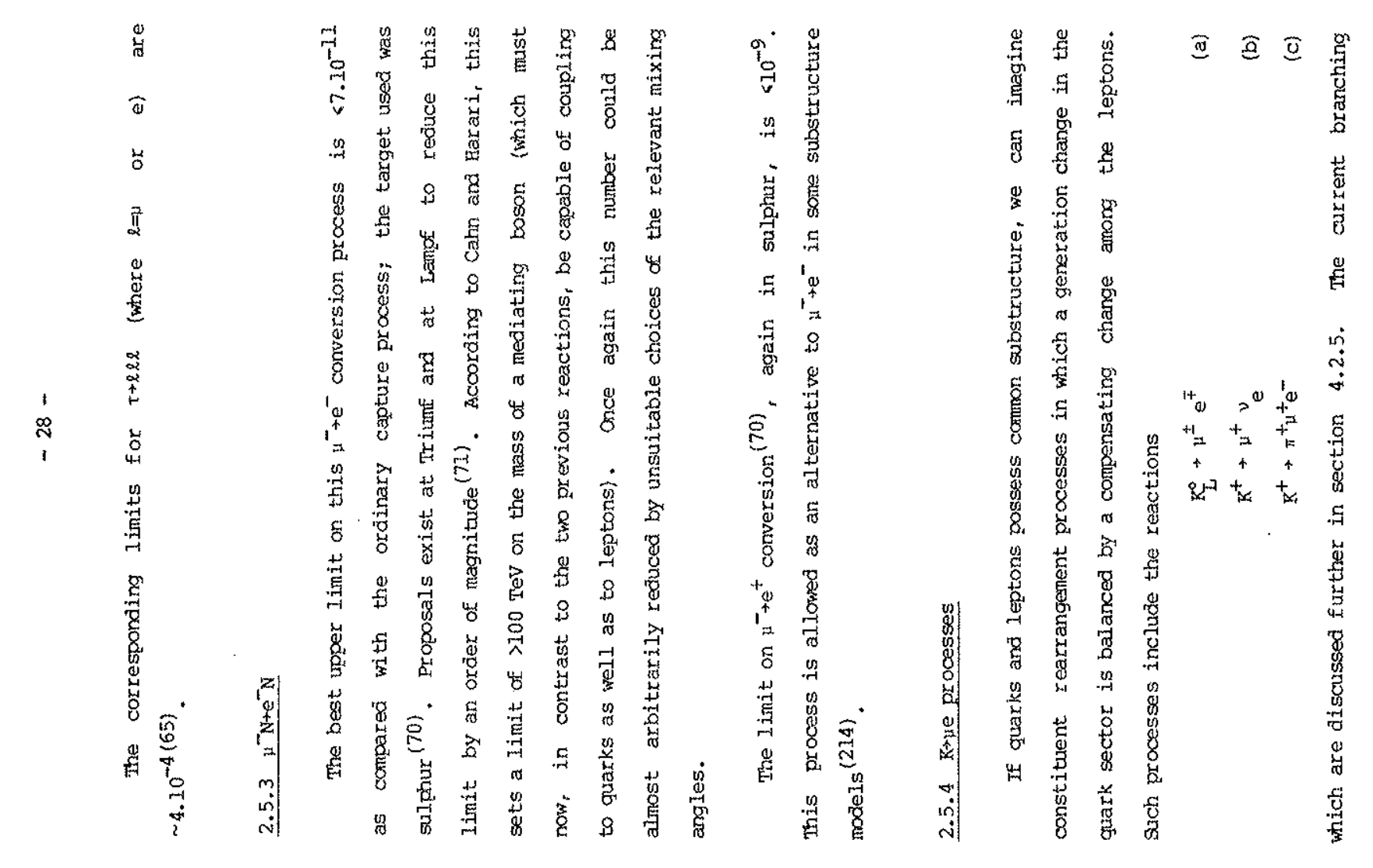

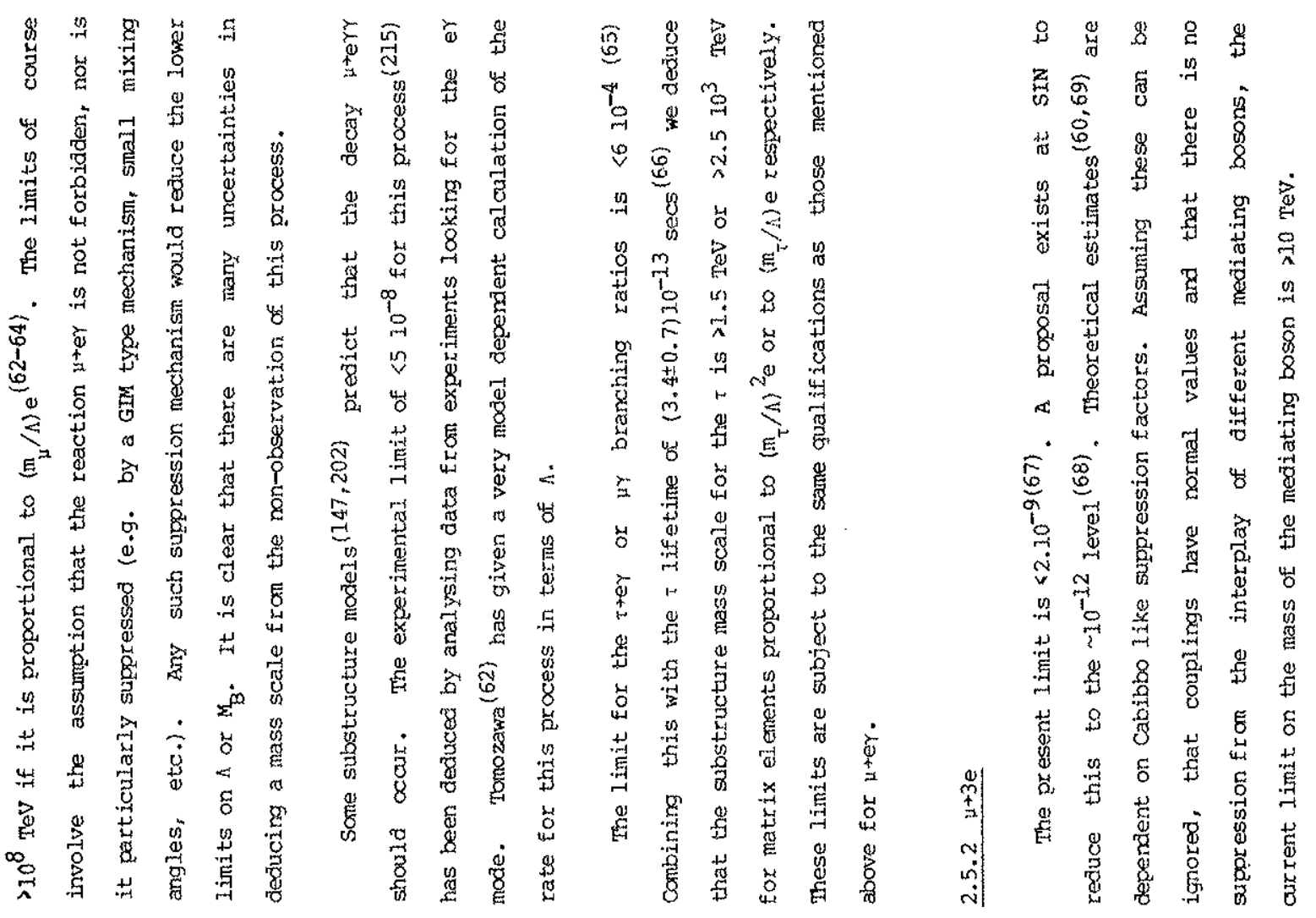




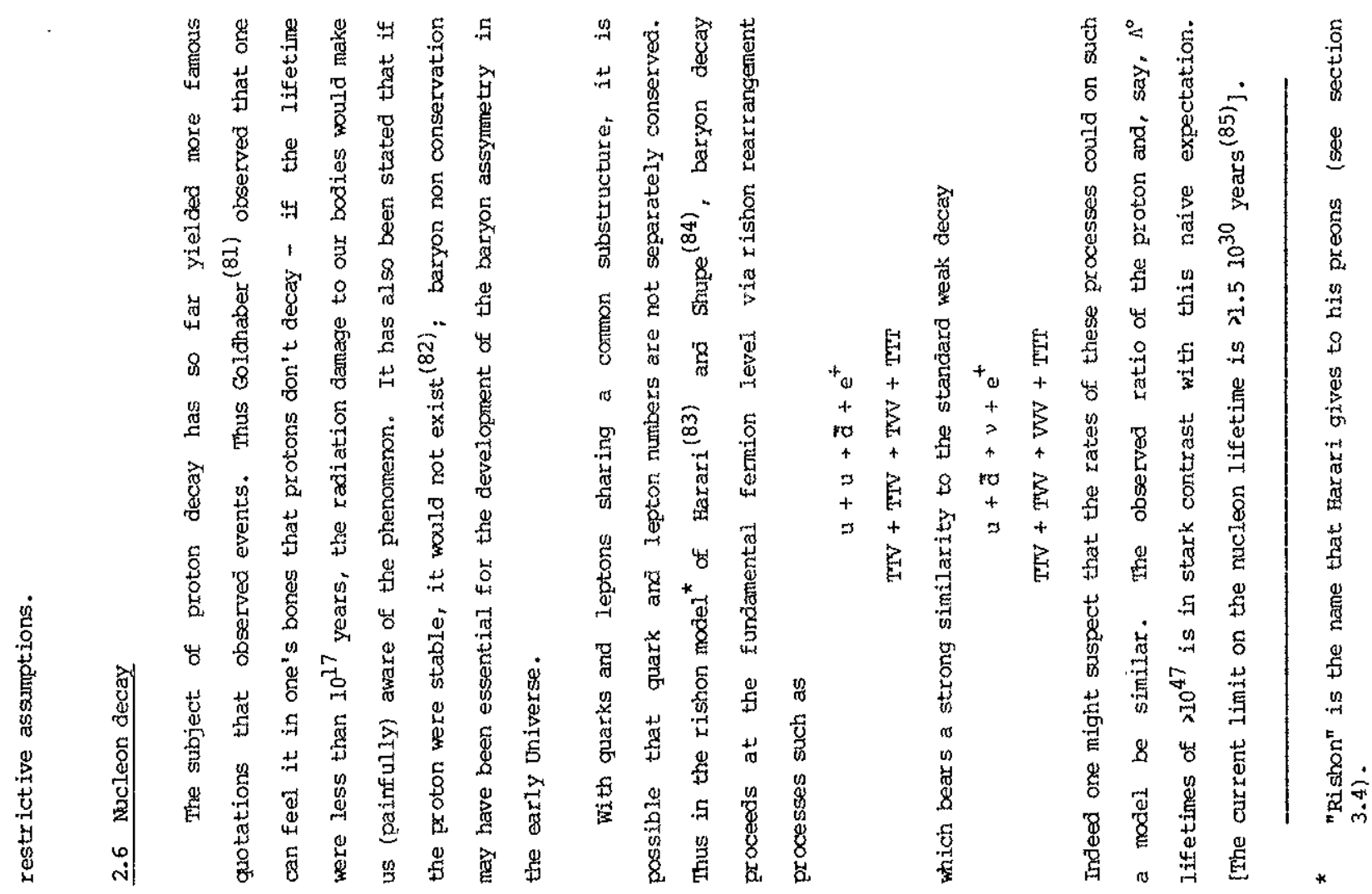

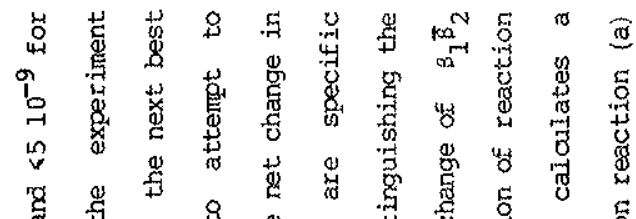

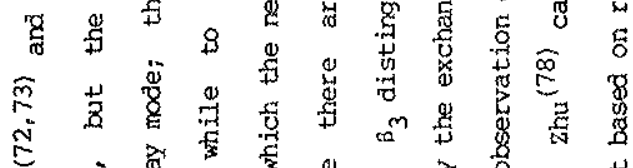

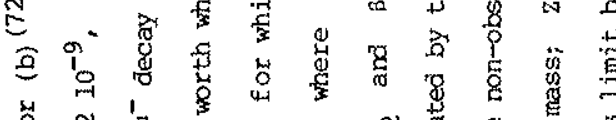

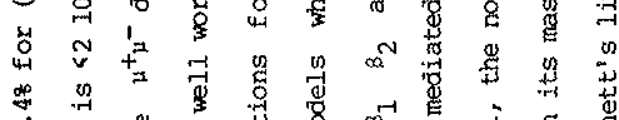

<

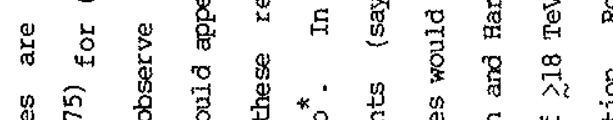

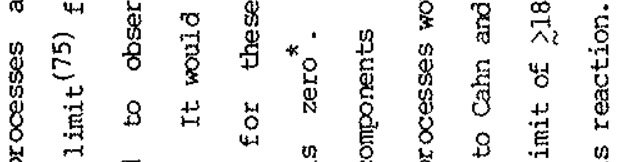

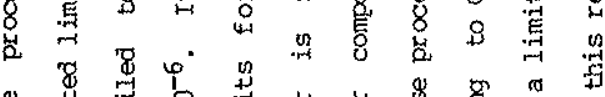

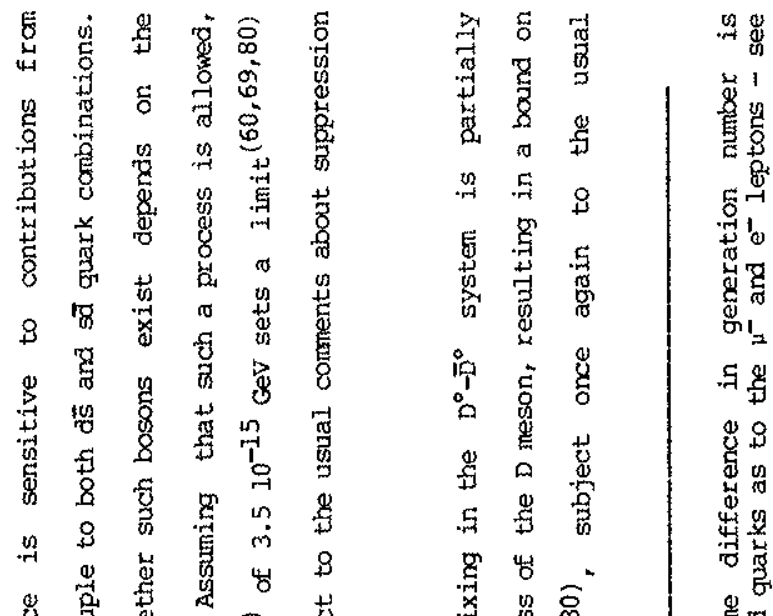

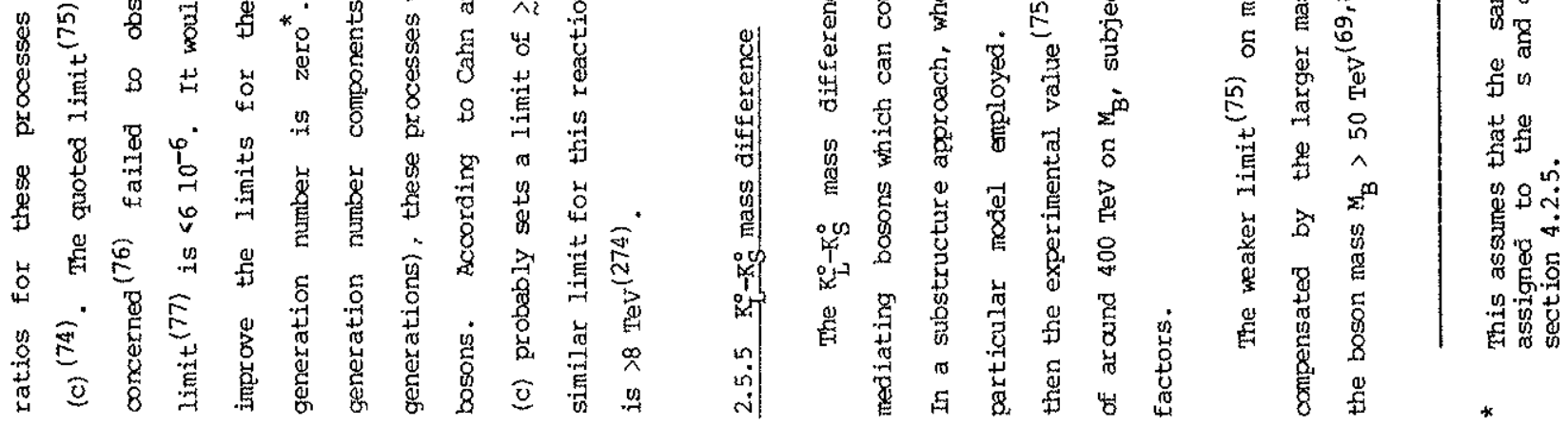




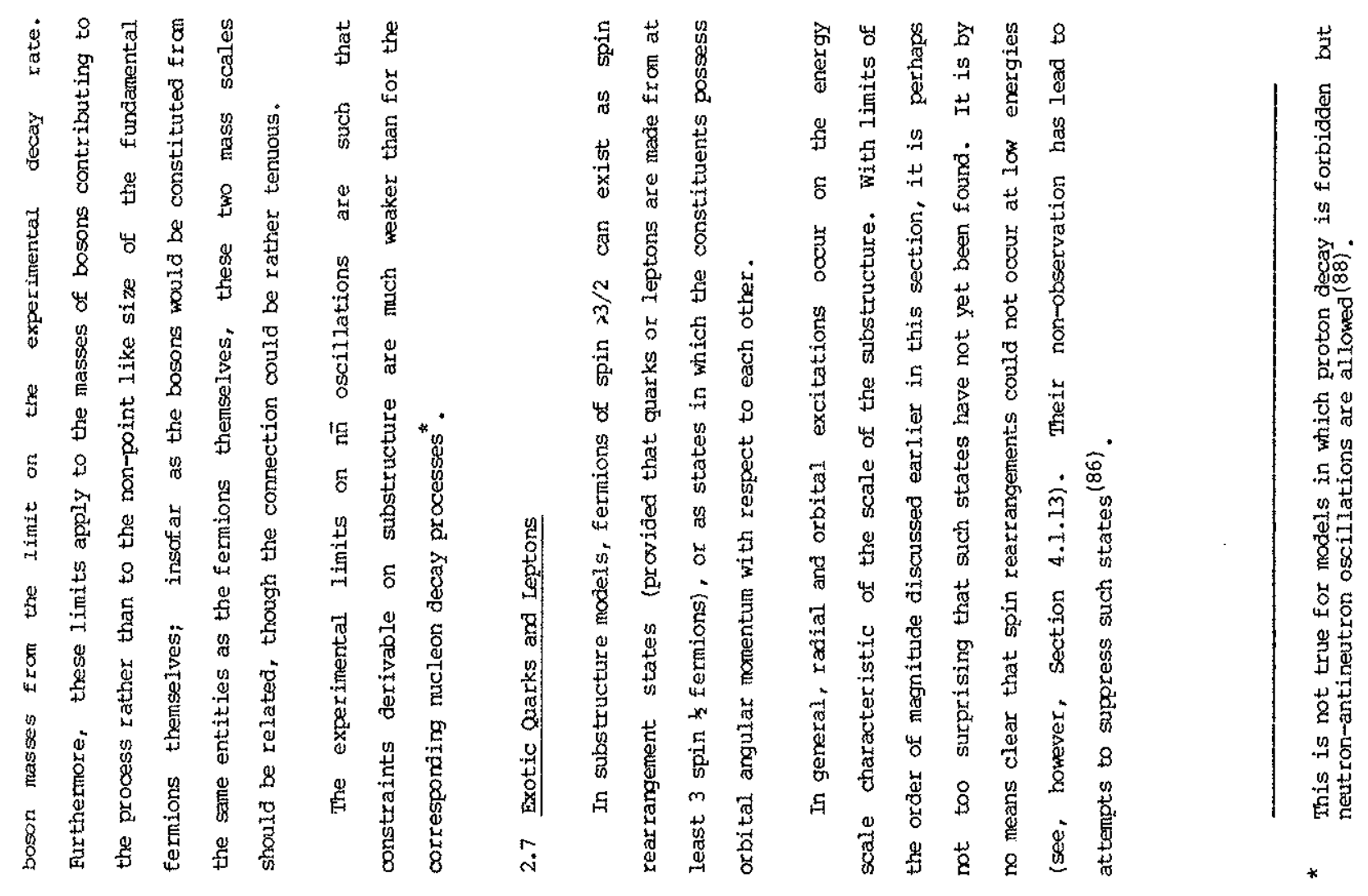

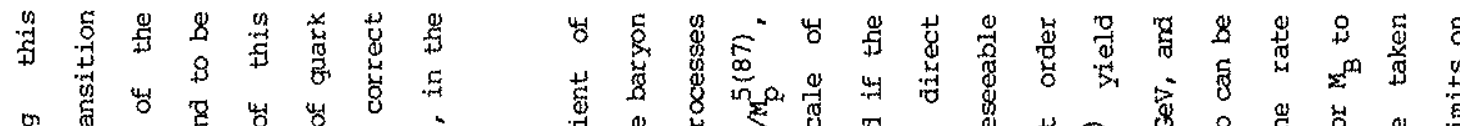

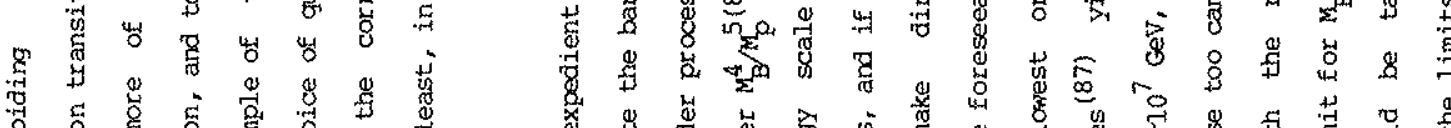

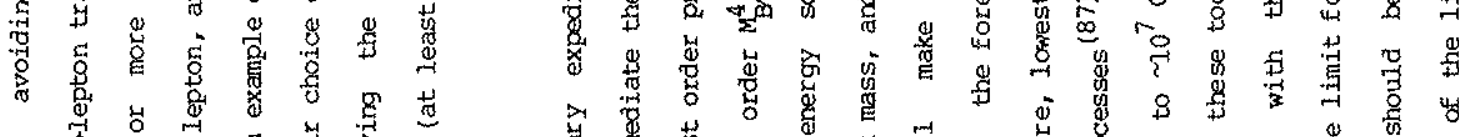

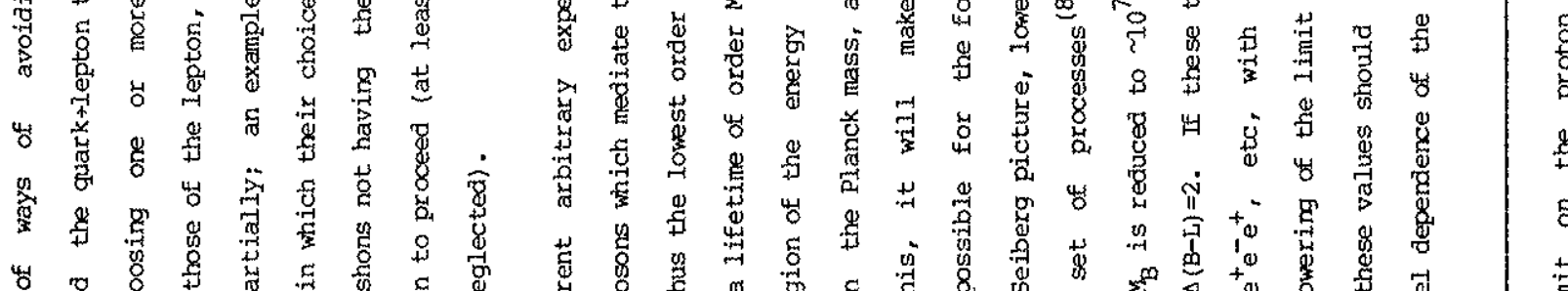

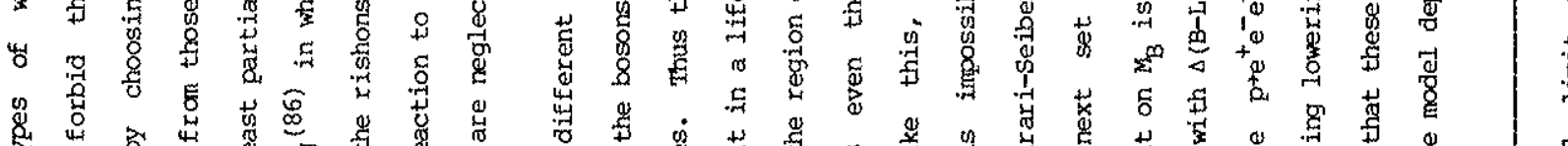

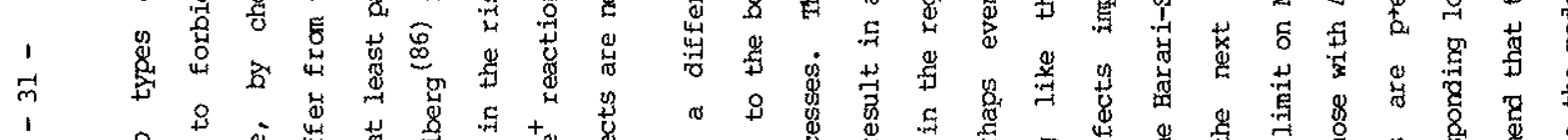

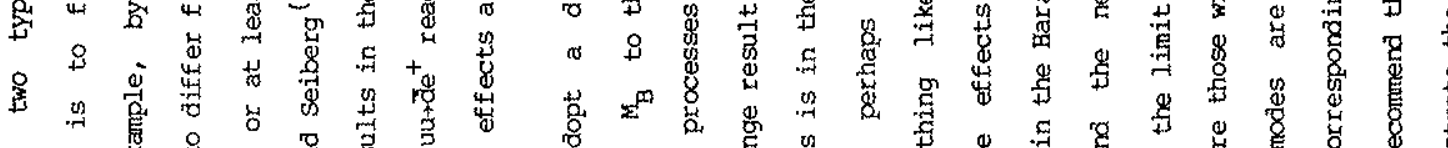

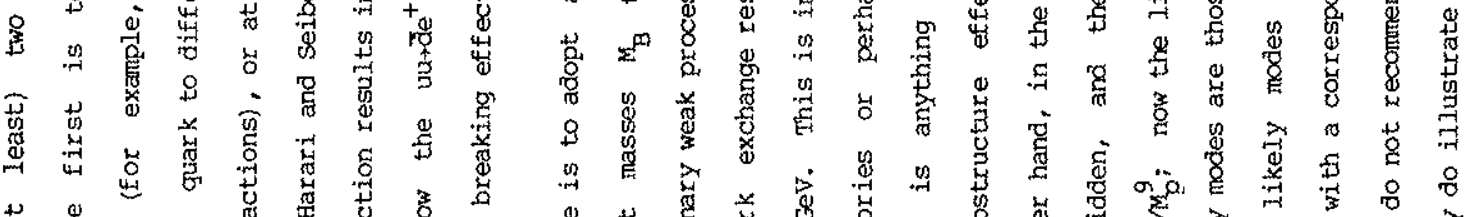

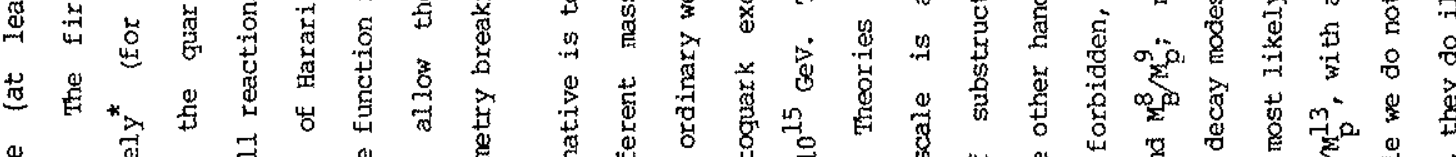

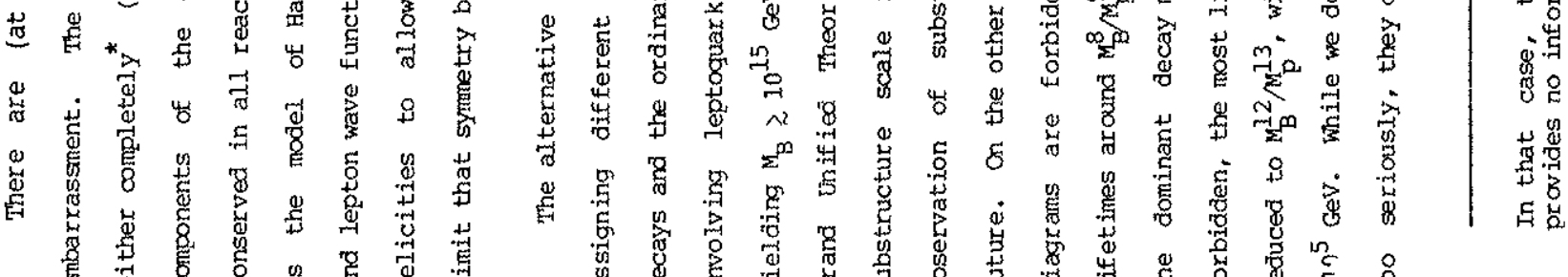

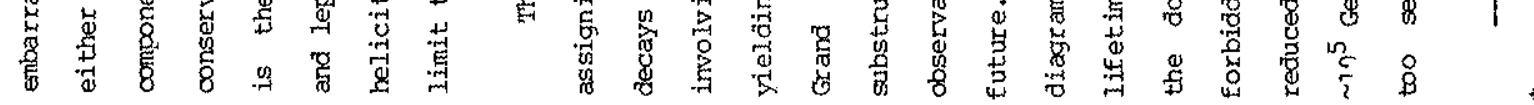




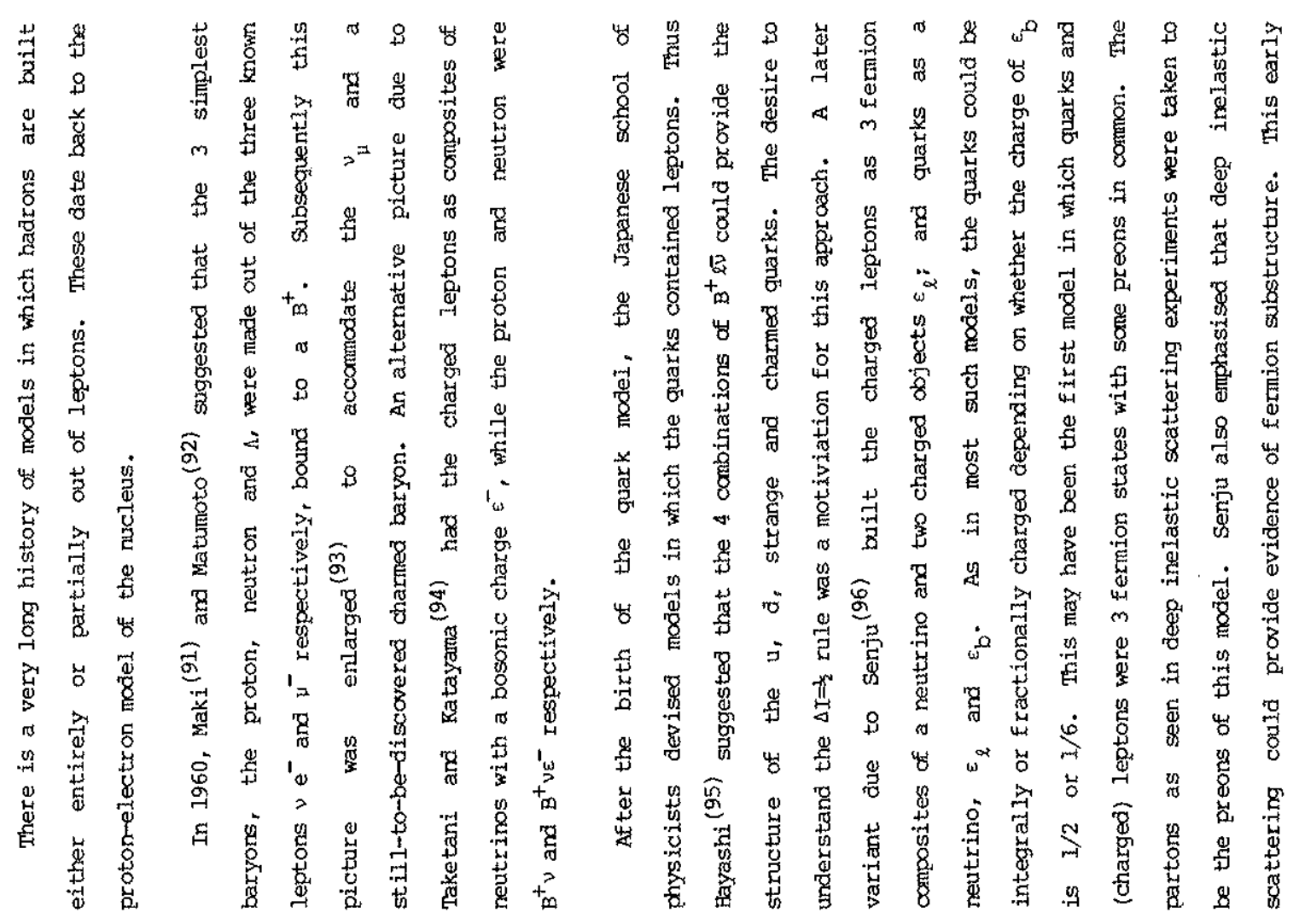

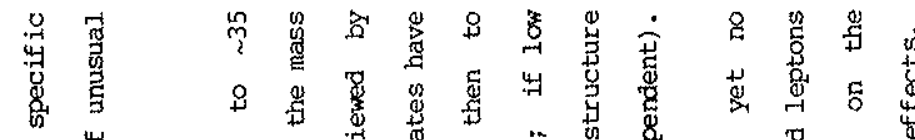

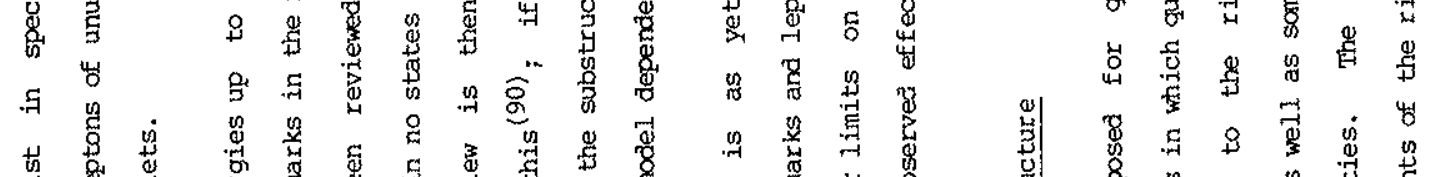

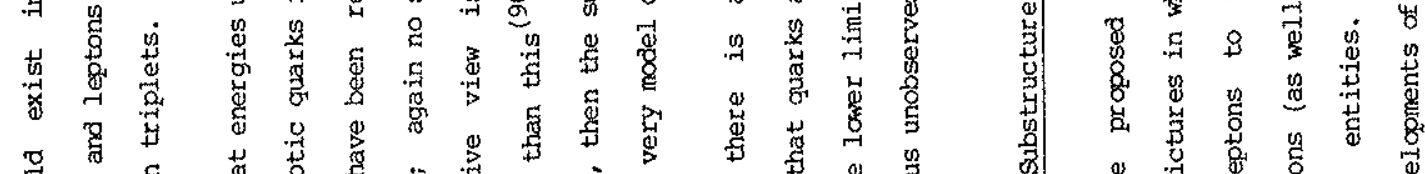

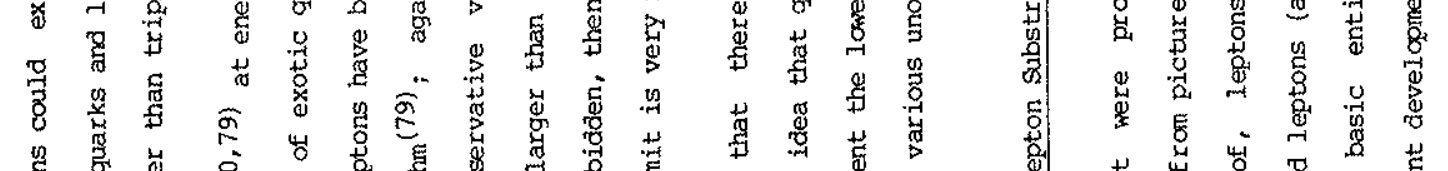

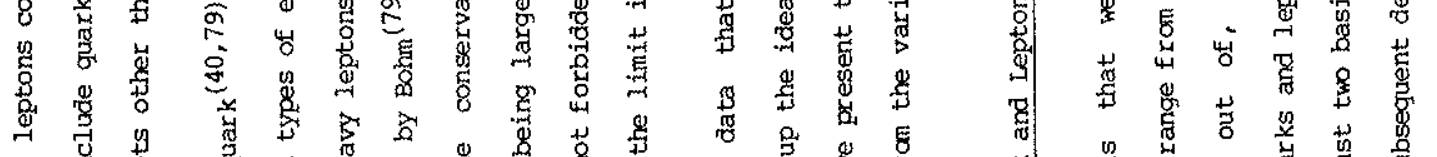

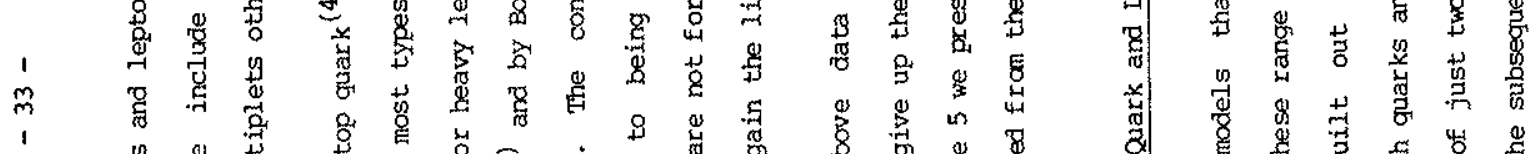

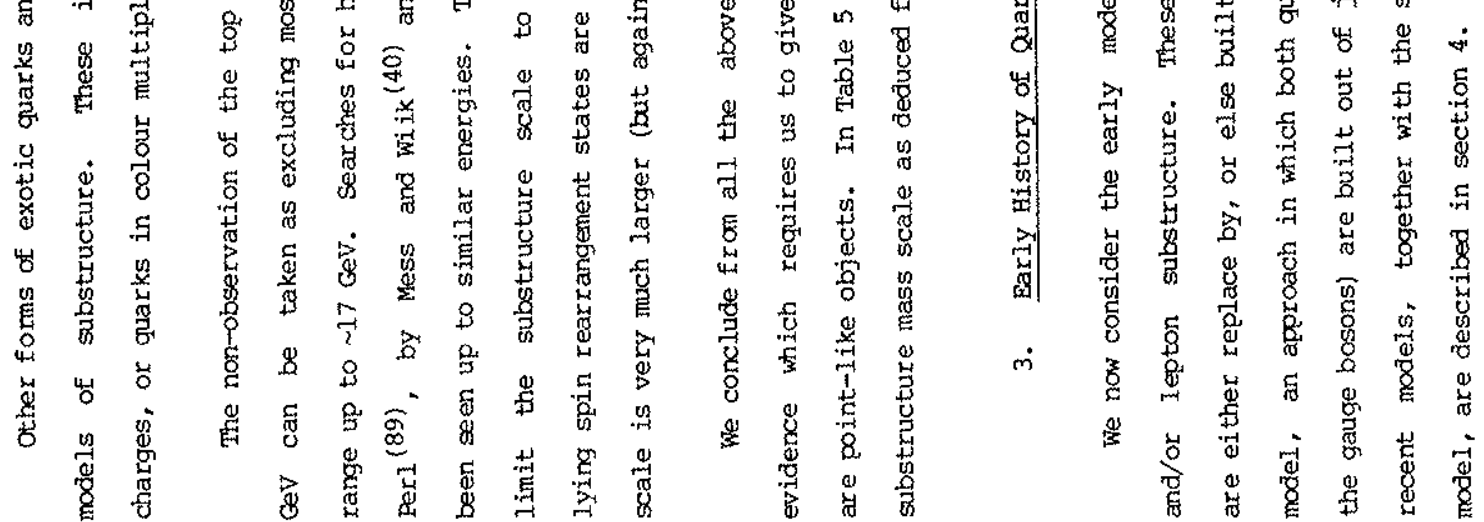

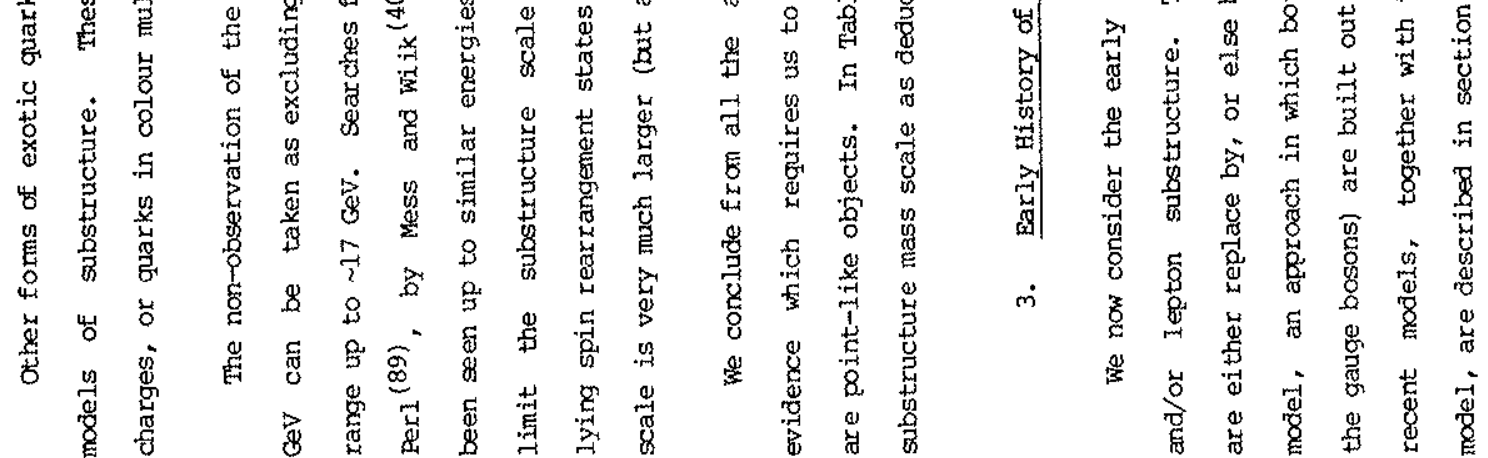




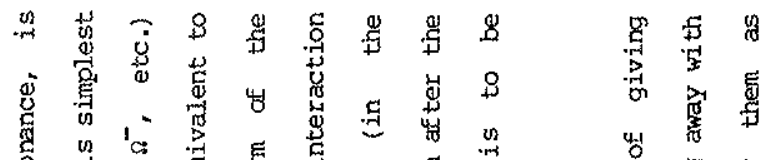

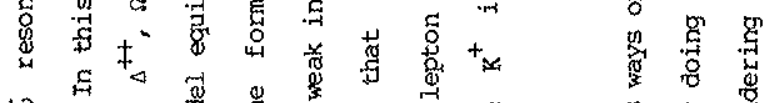

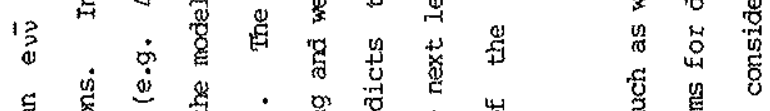

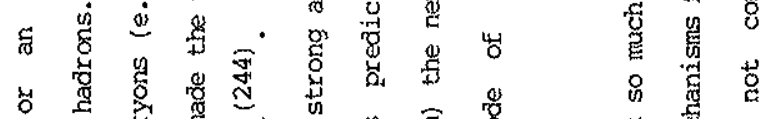

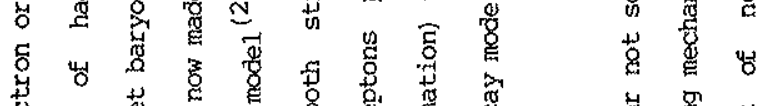

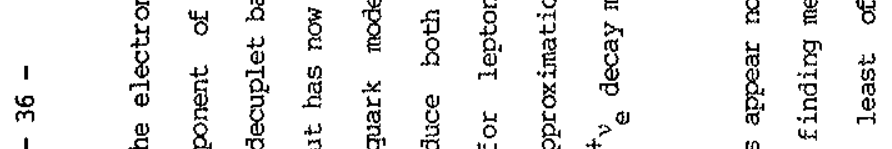

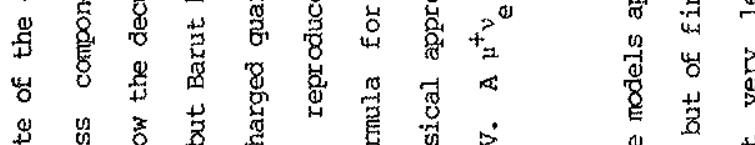

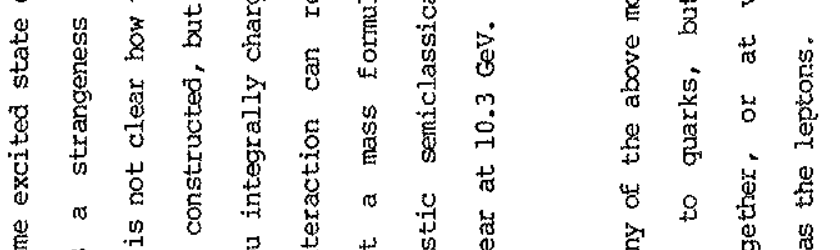

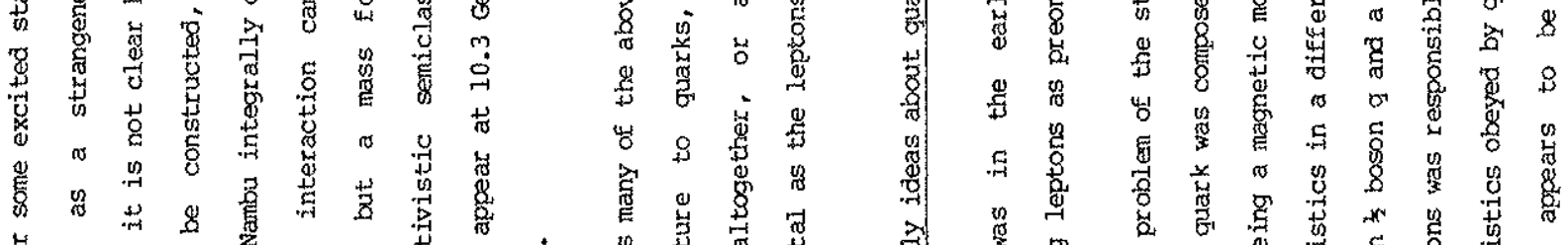

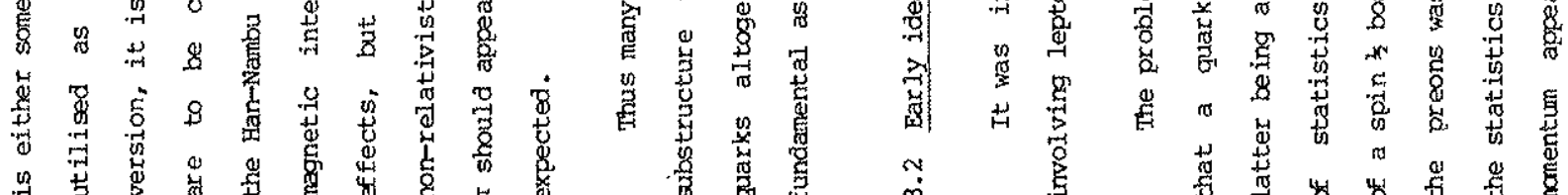

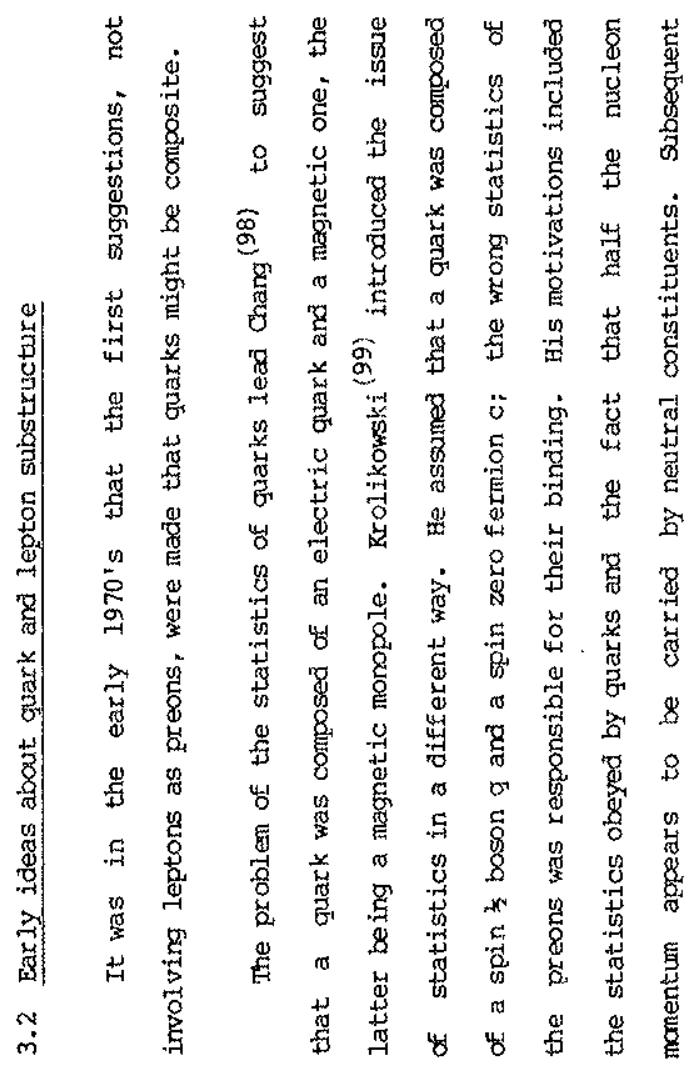

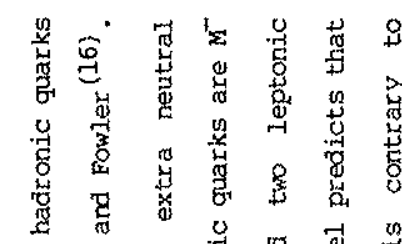

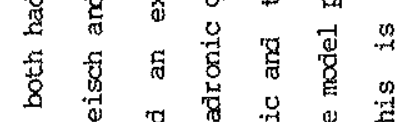

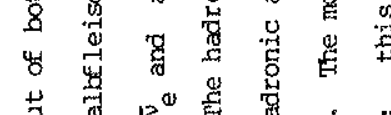

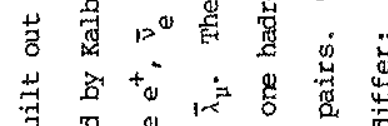

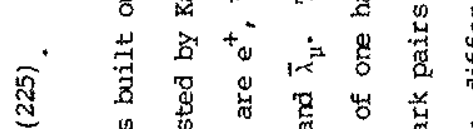

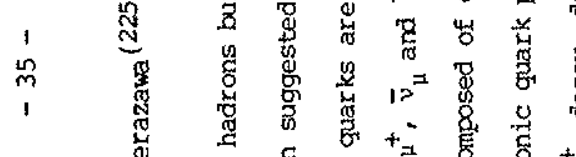

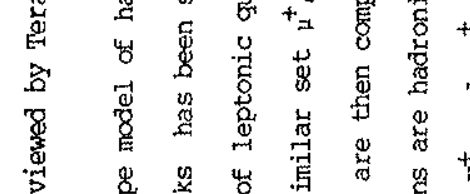

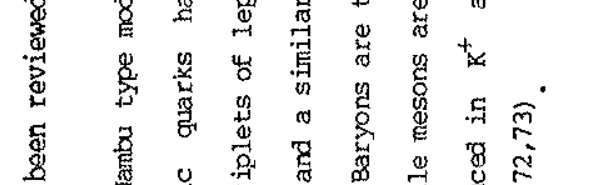

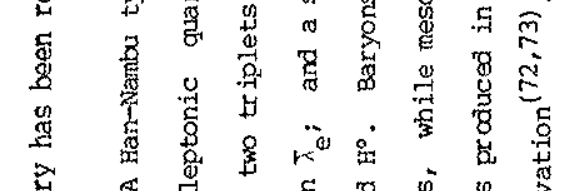

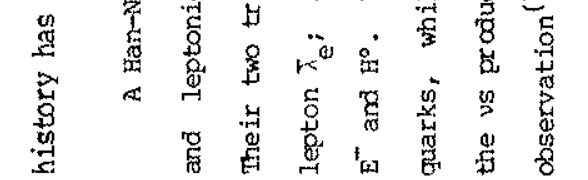

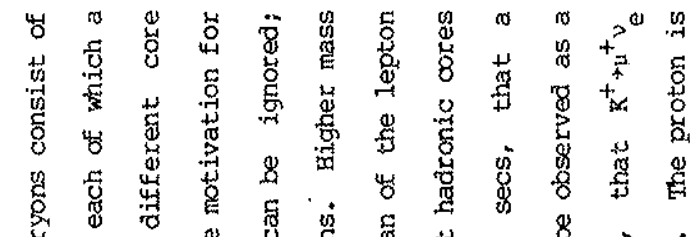

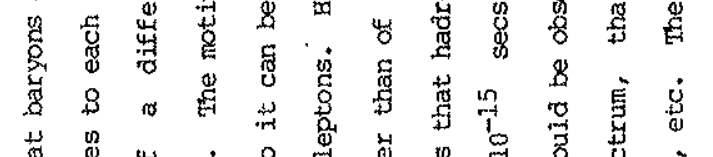

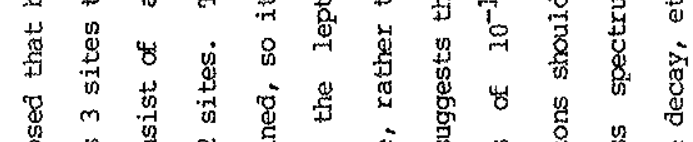

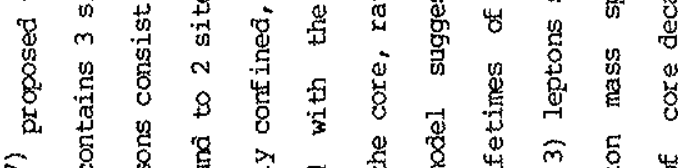

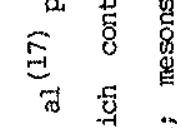

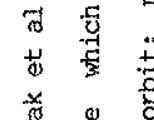

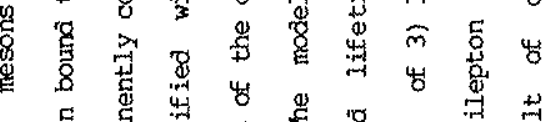

41

章事

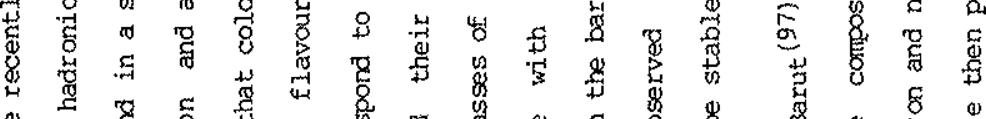

II)

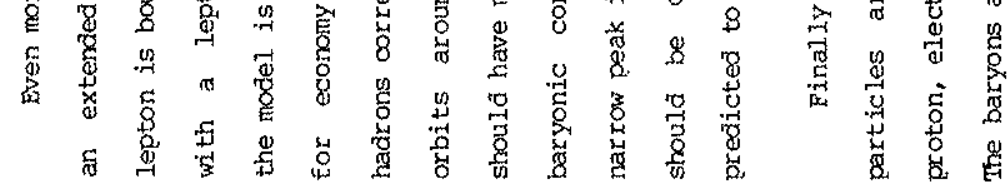




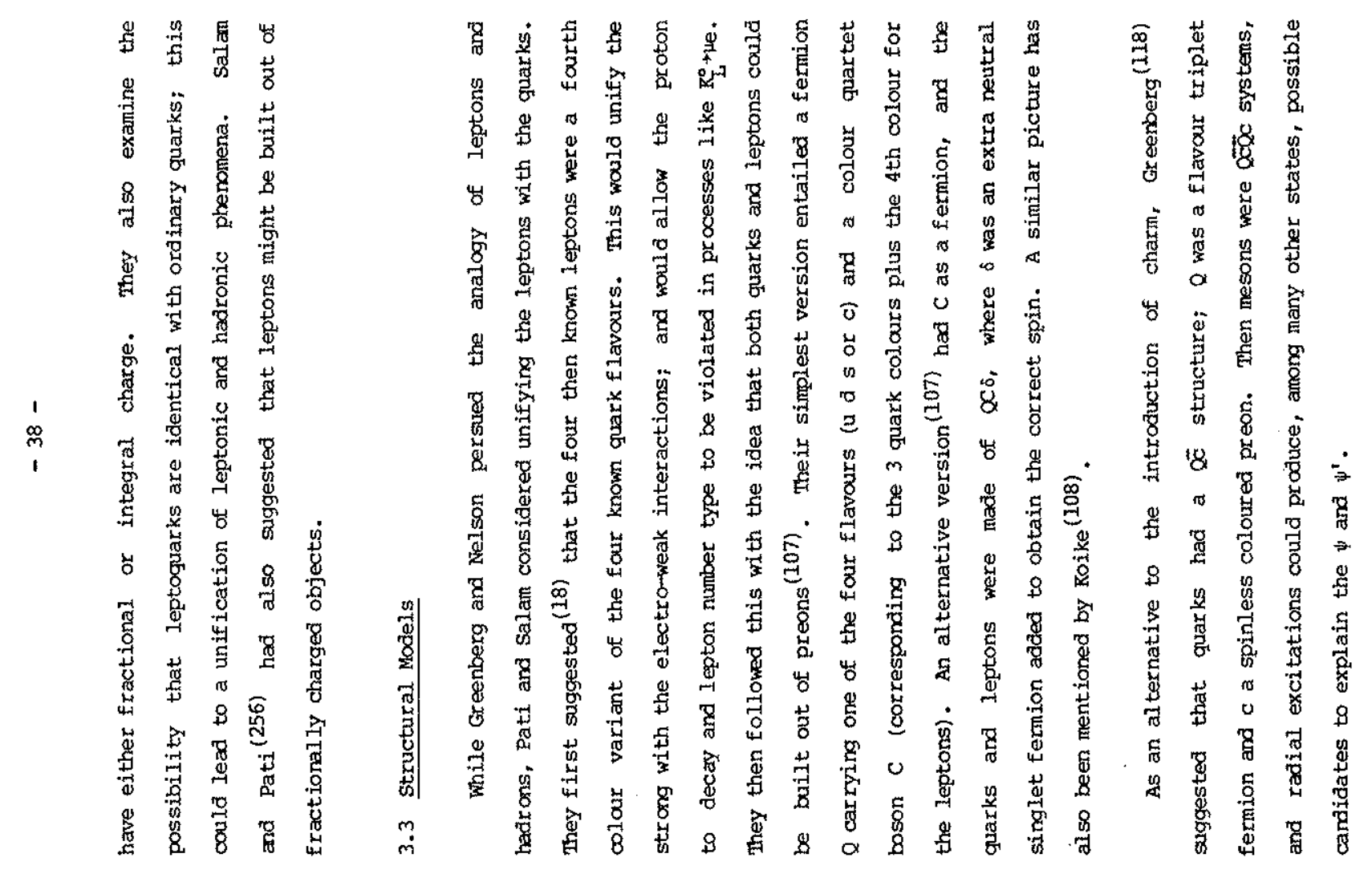

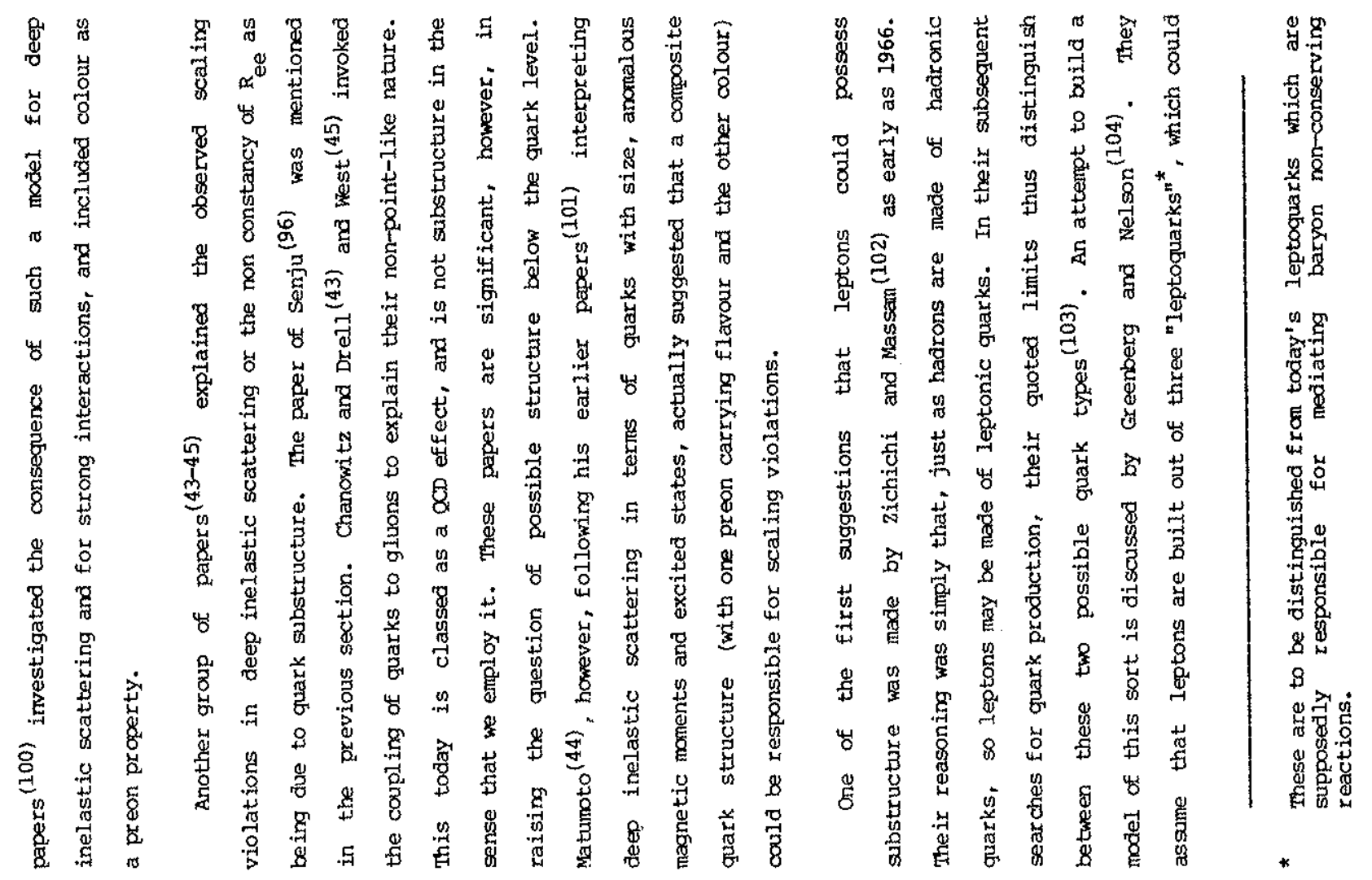



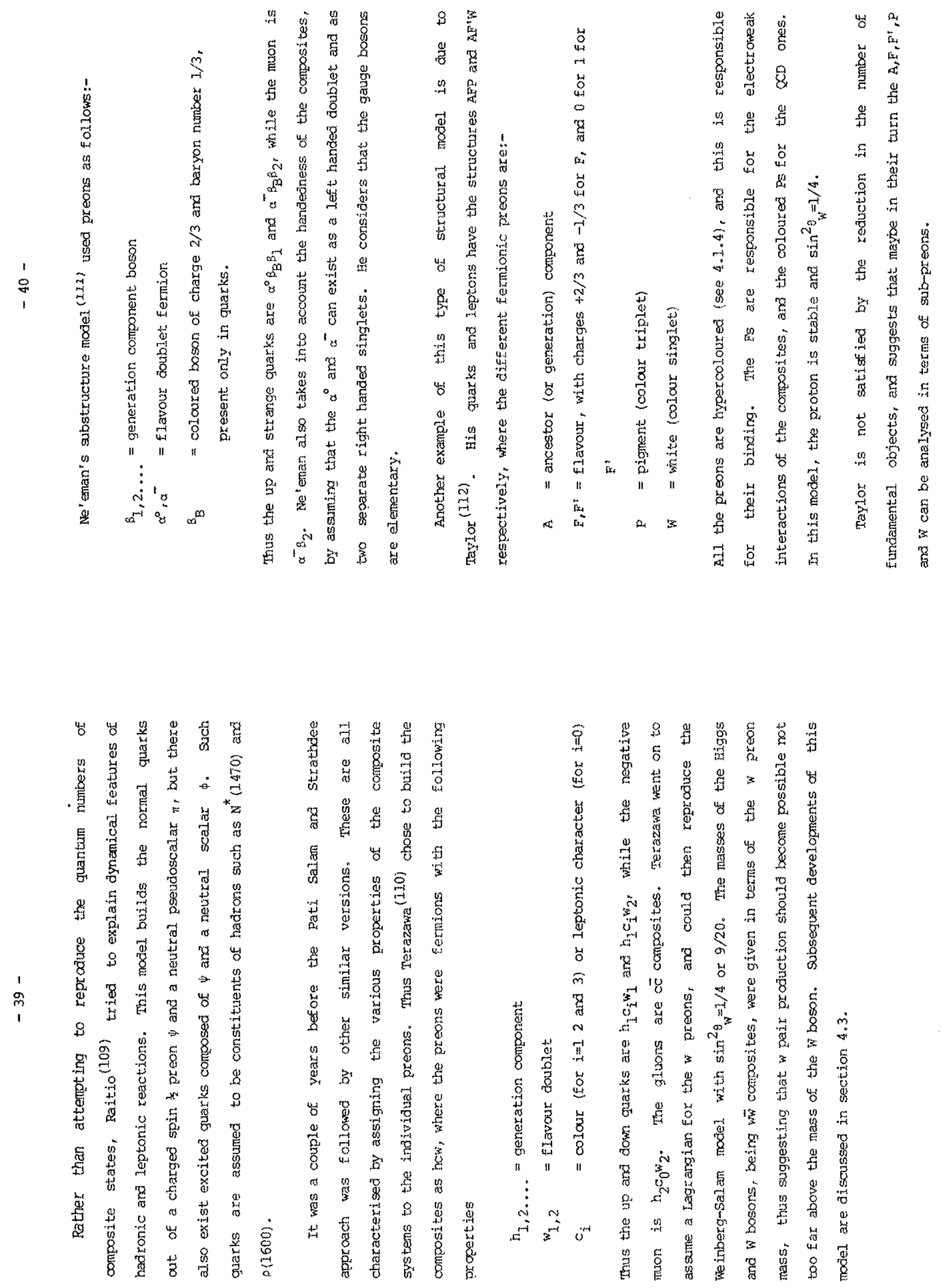

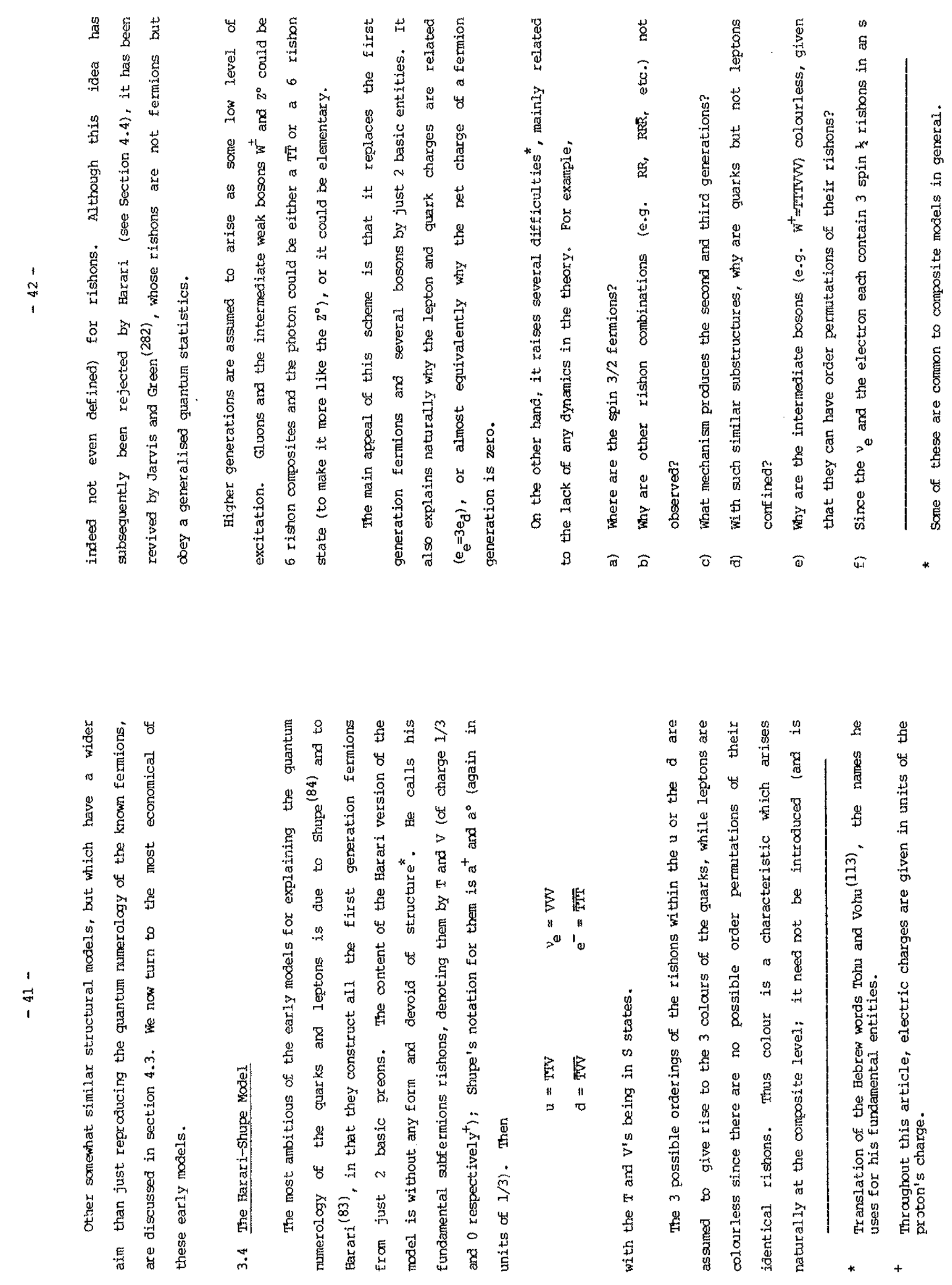

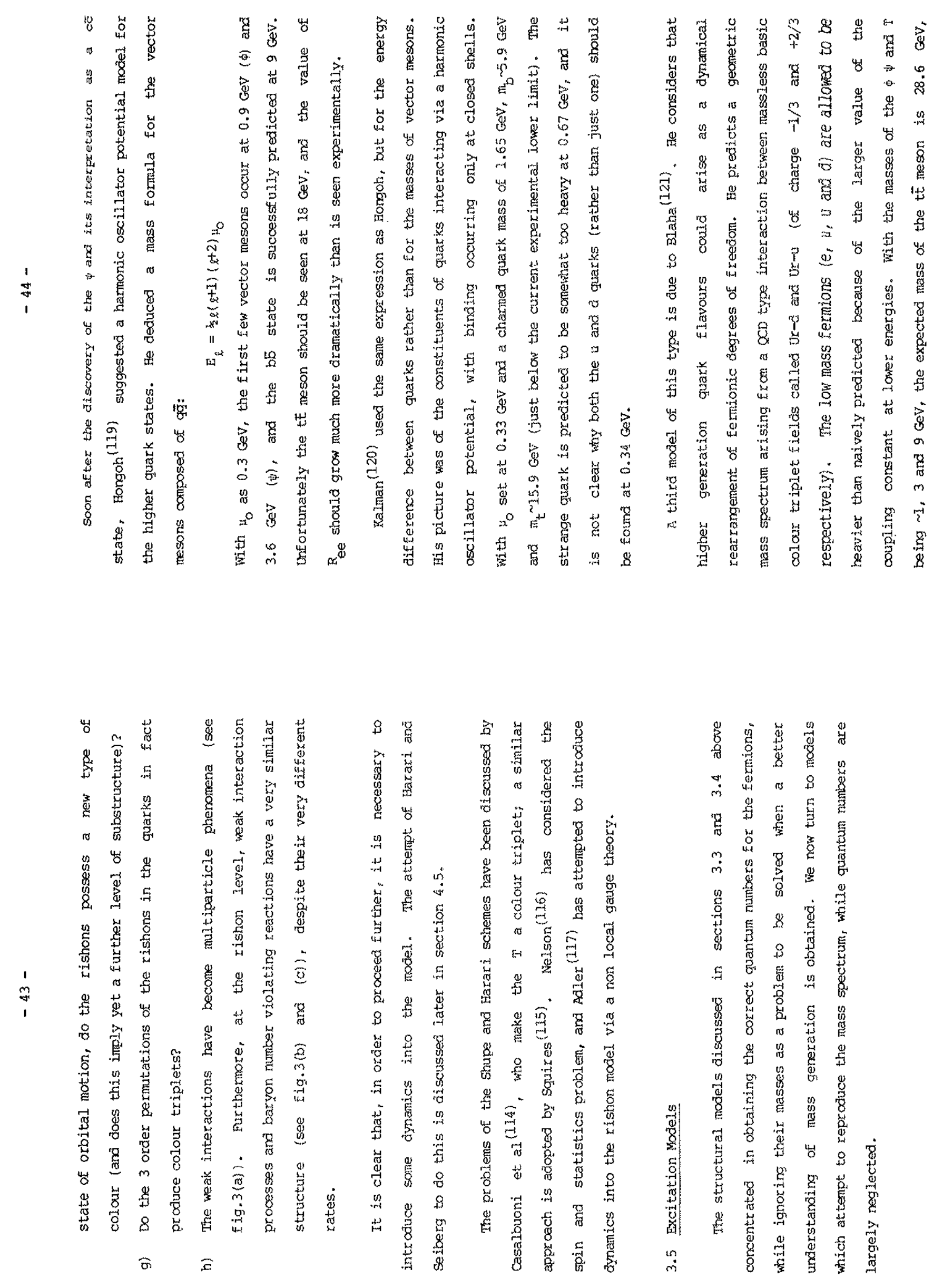

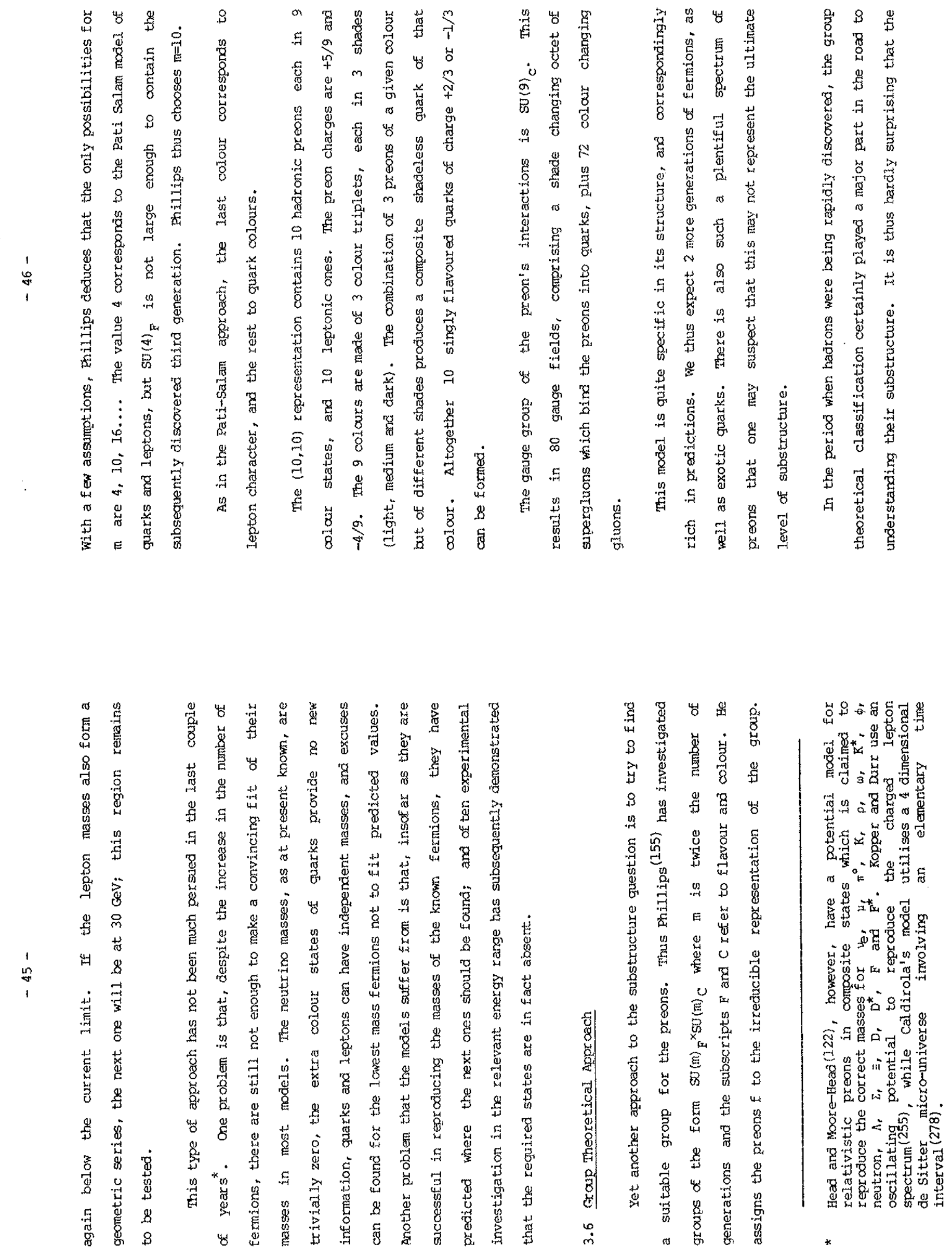


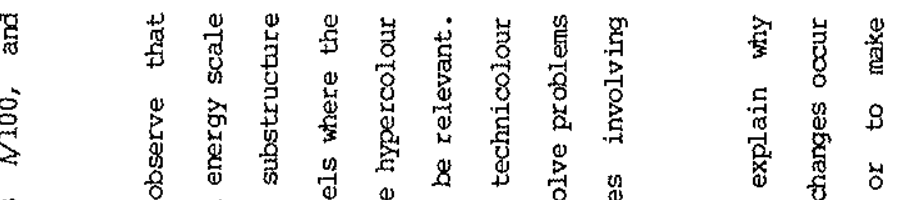

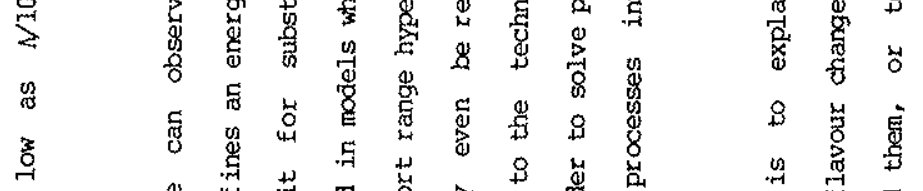

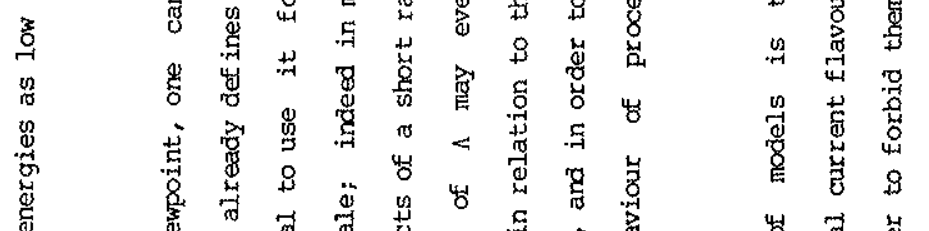

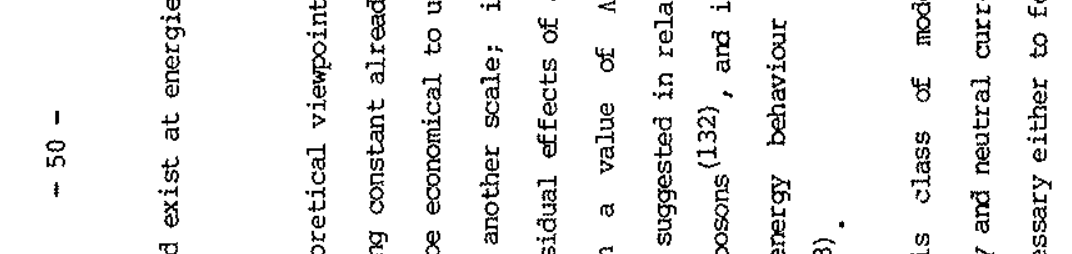

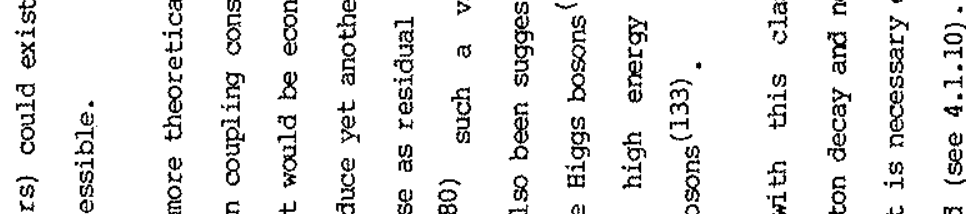

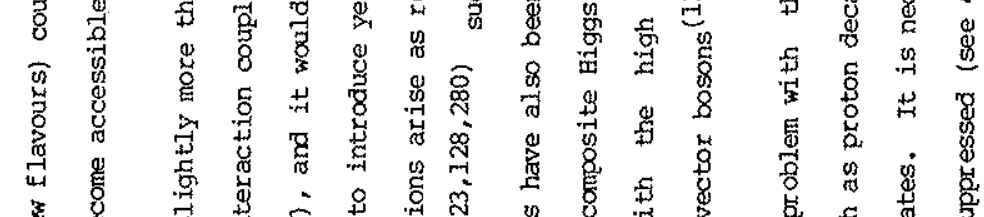

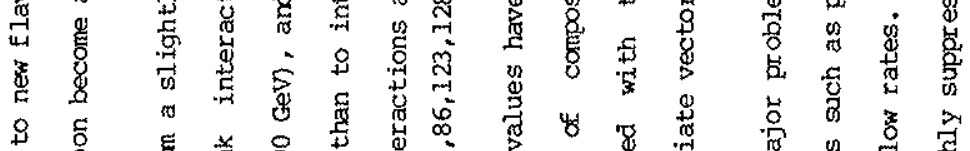

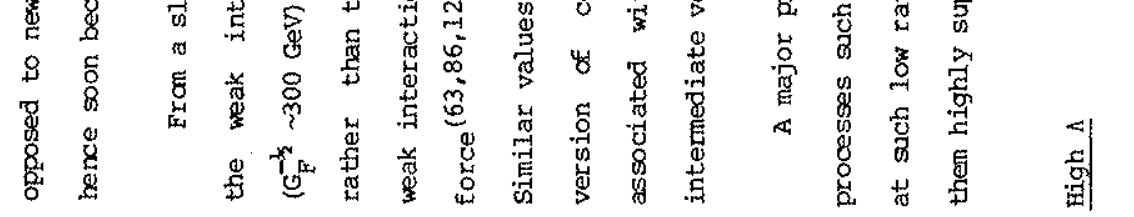

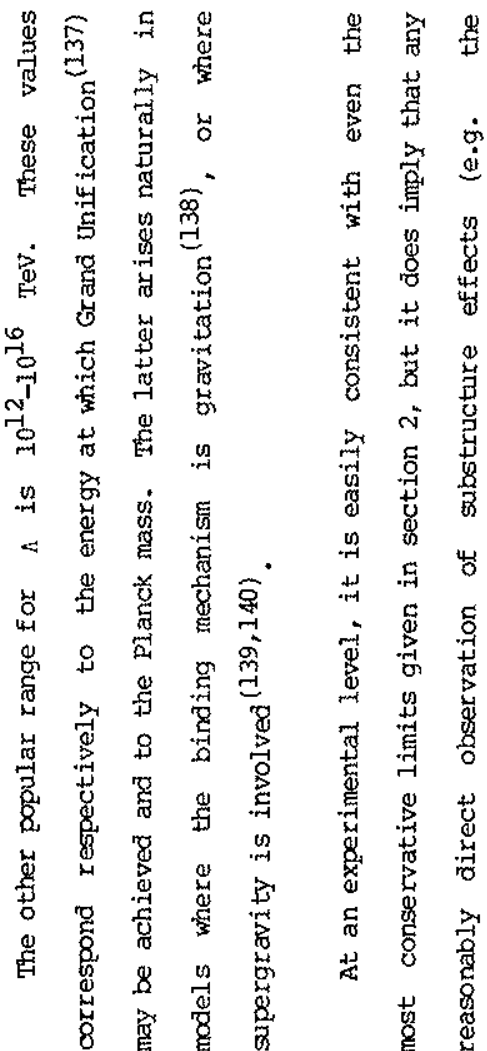

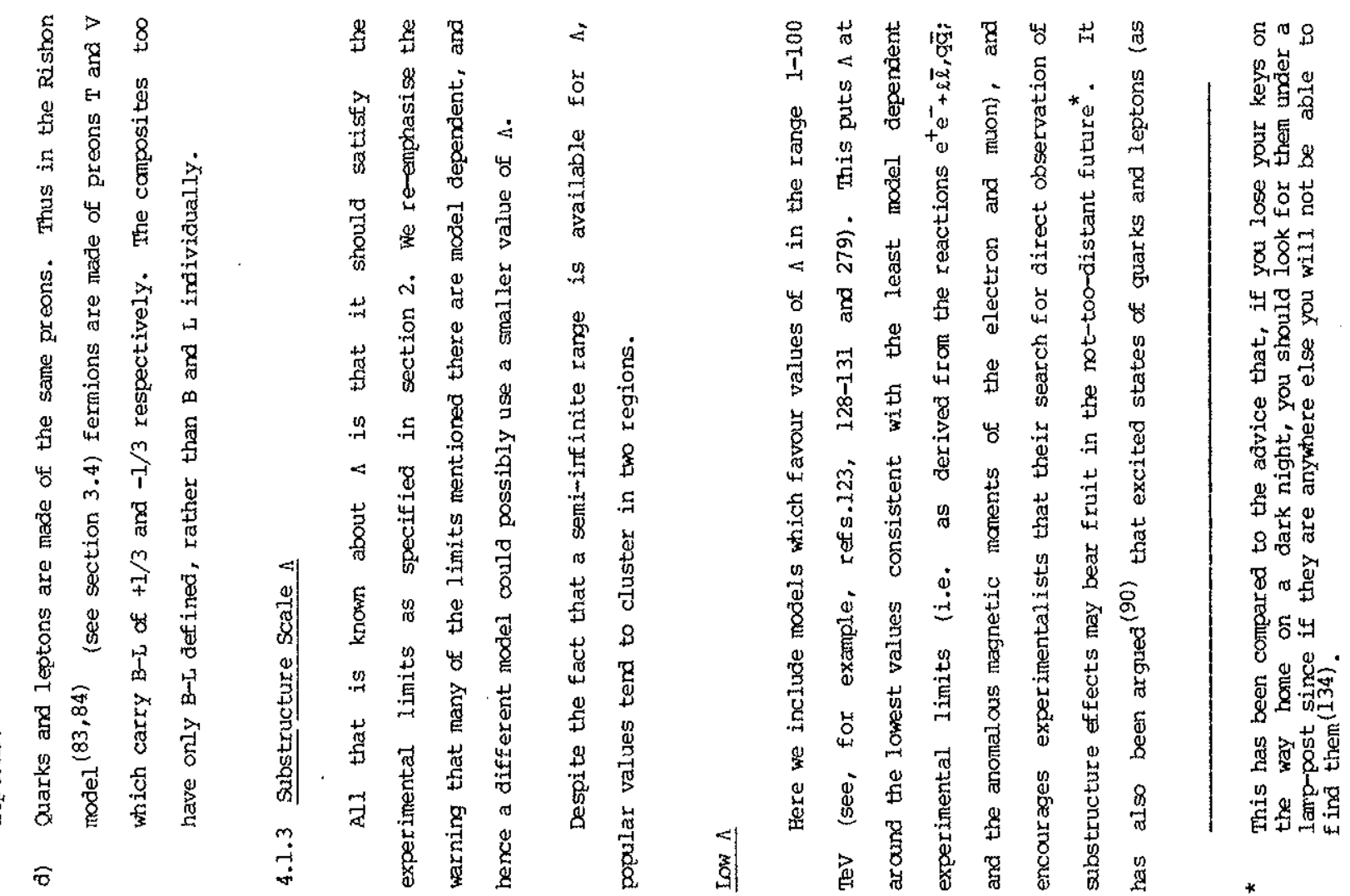



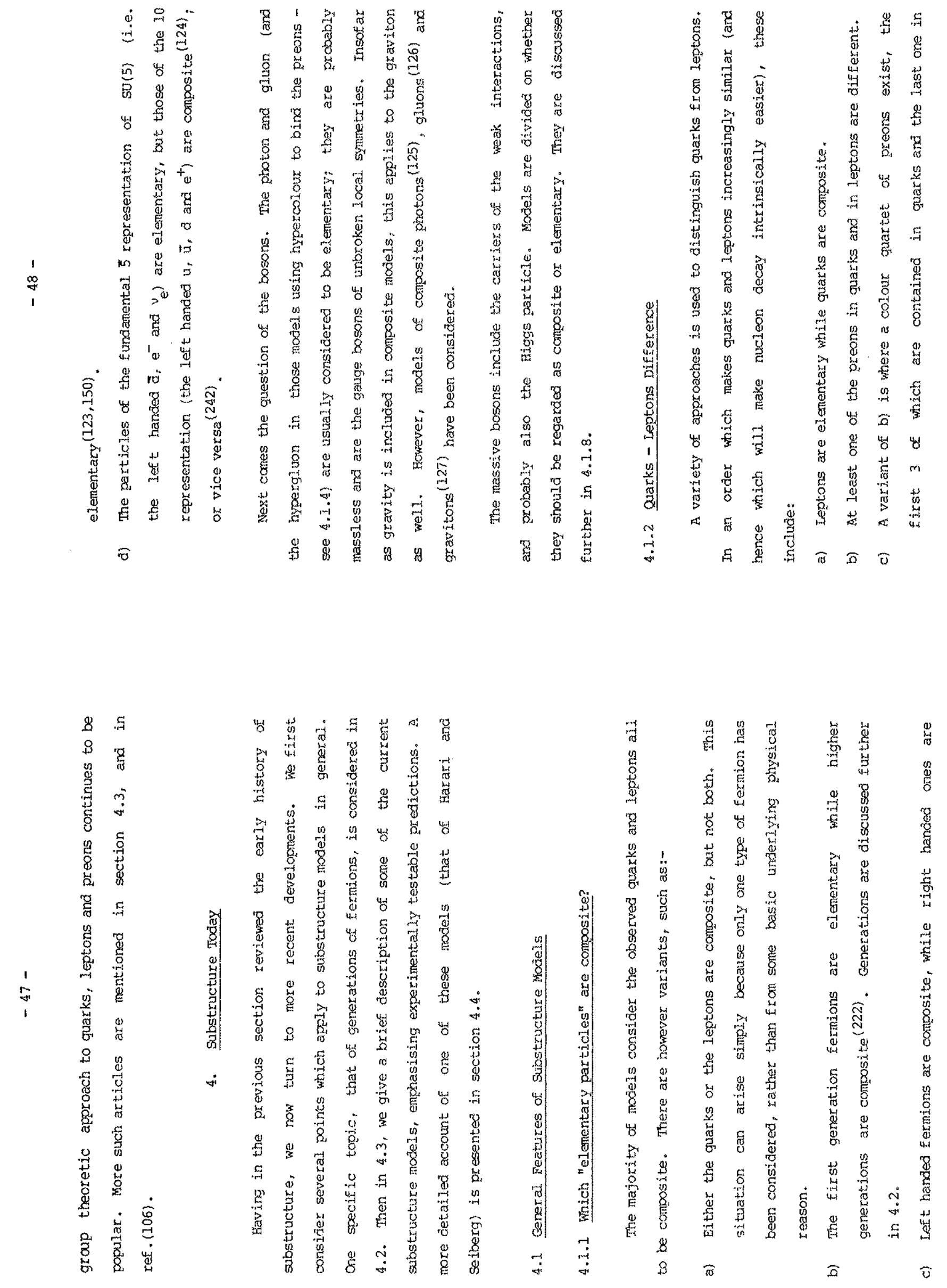

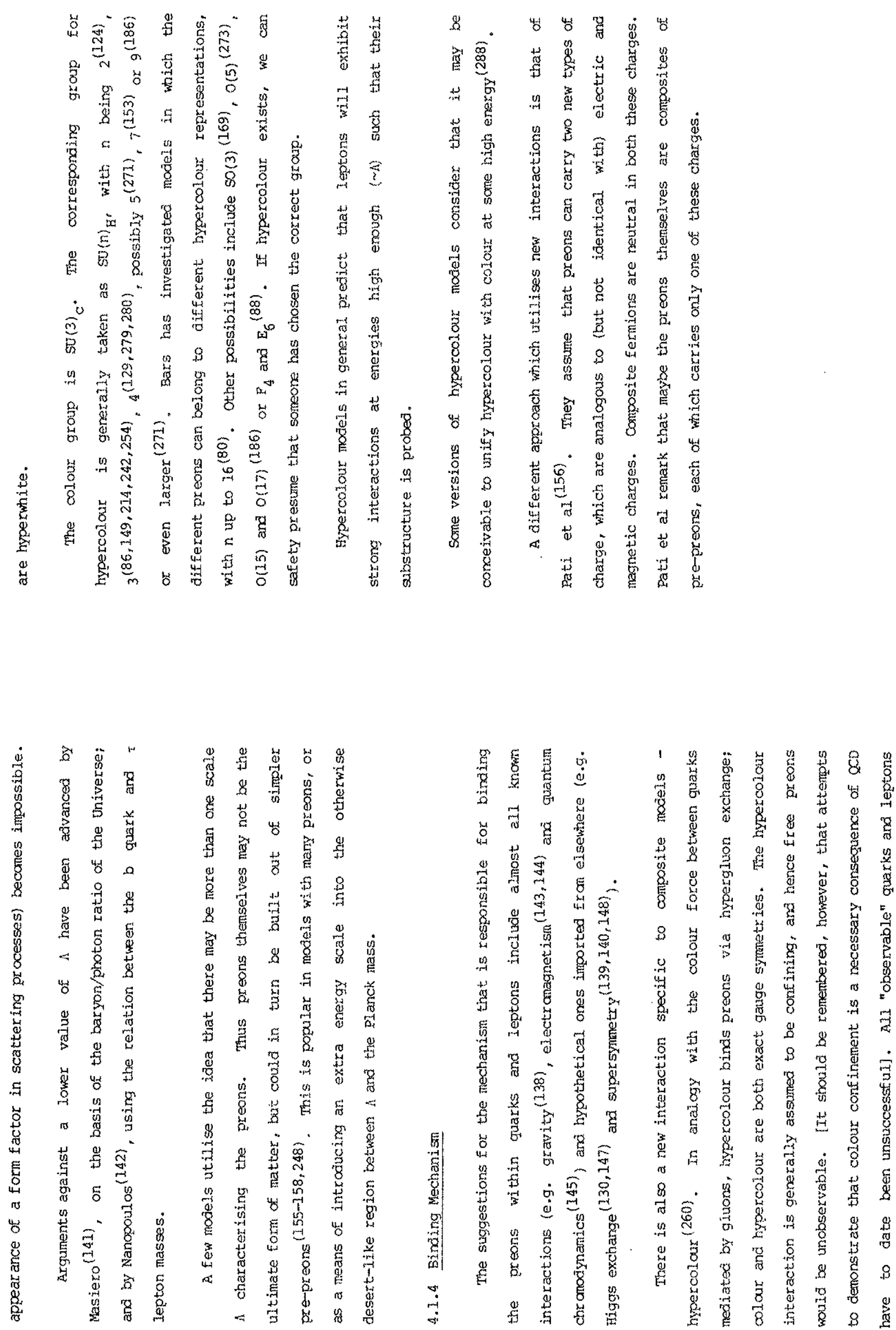

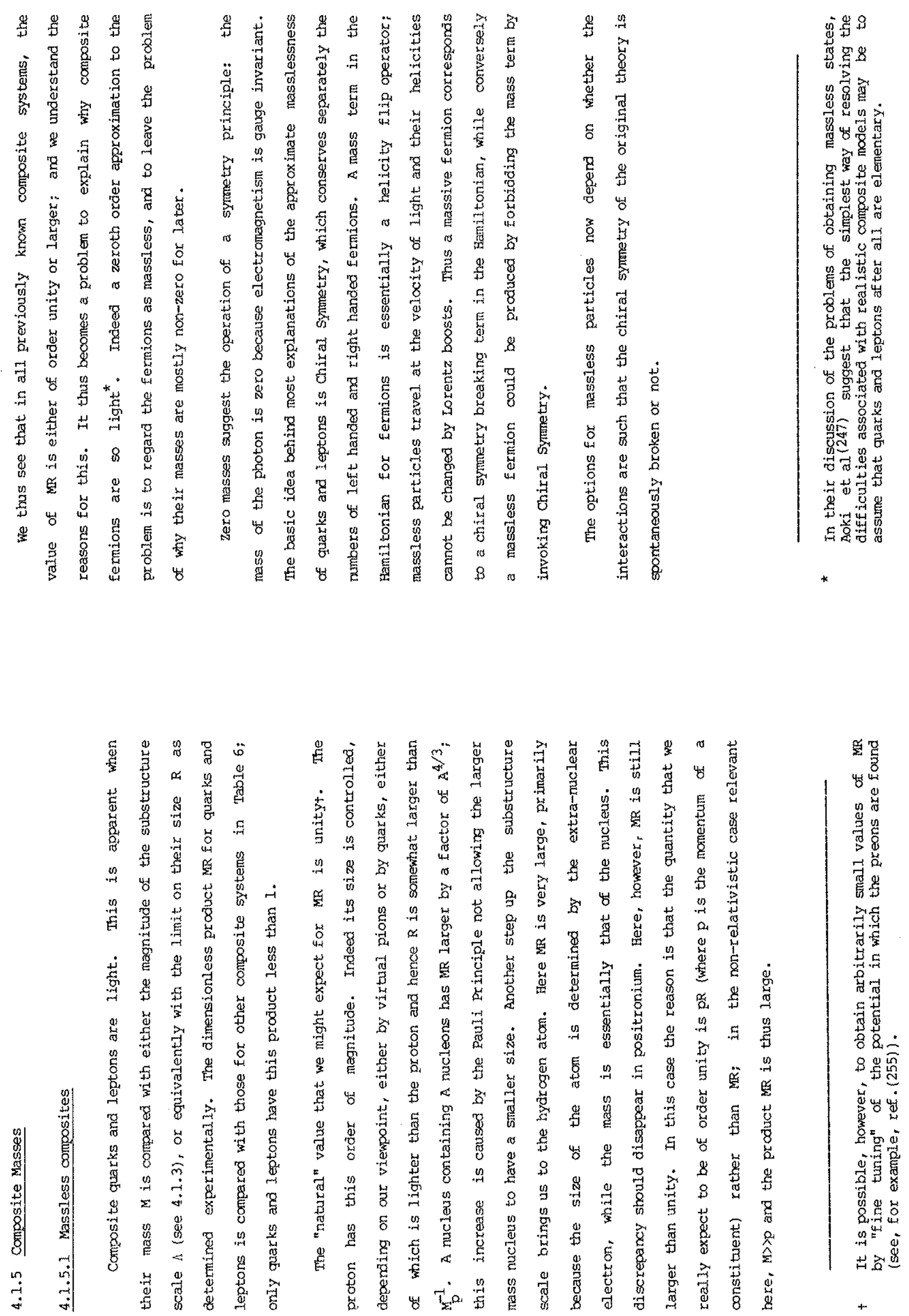


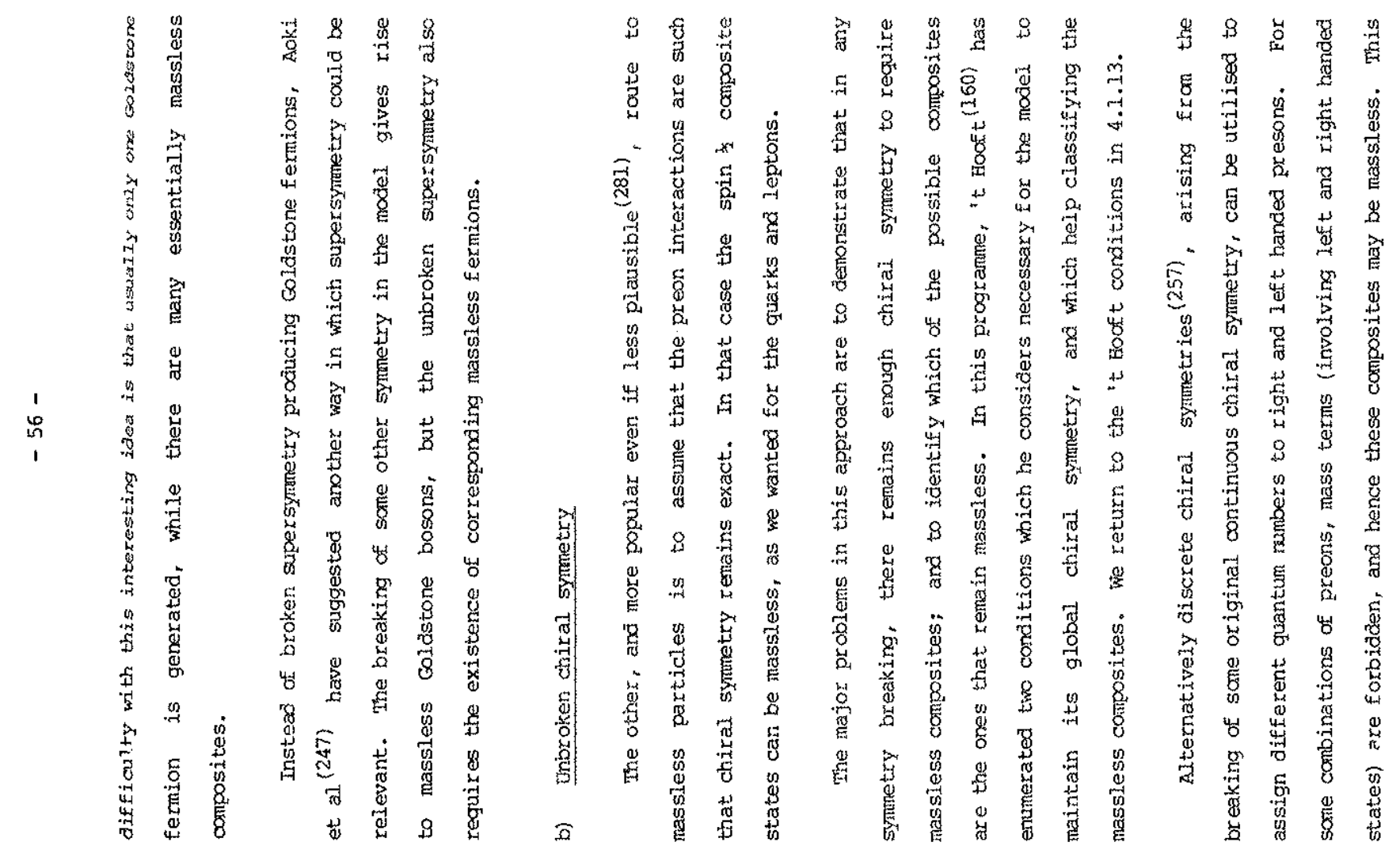

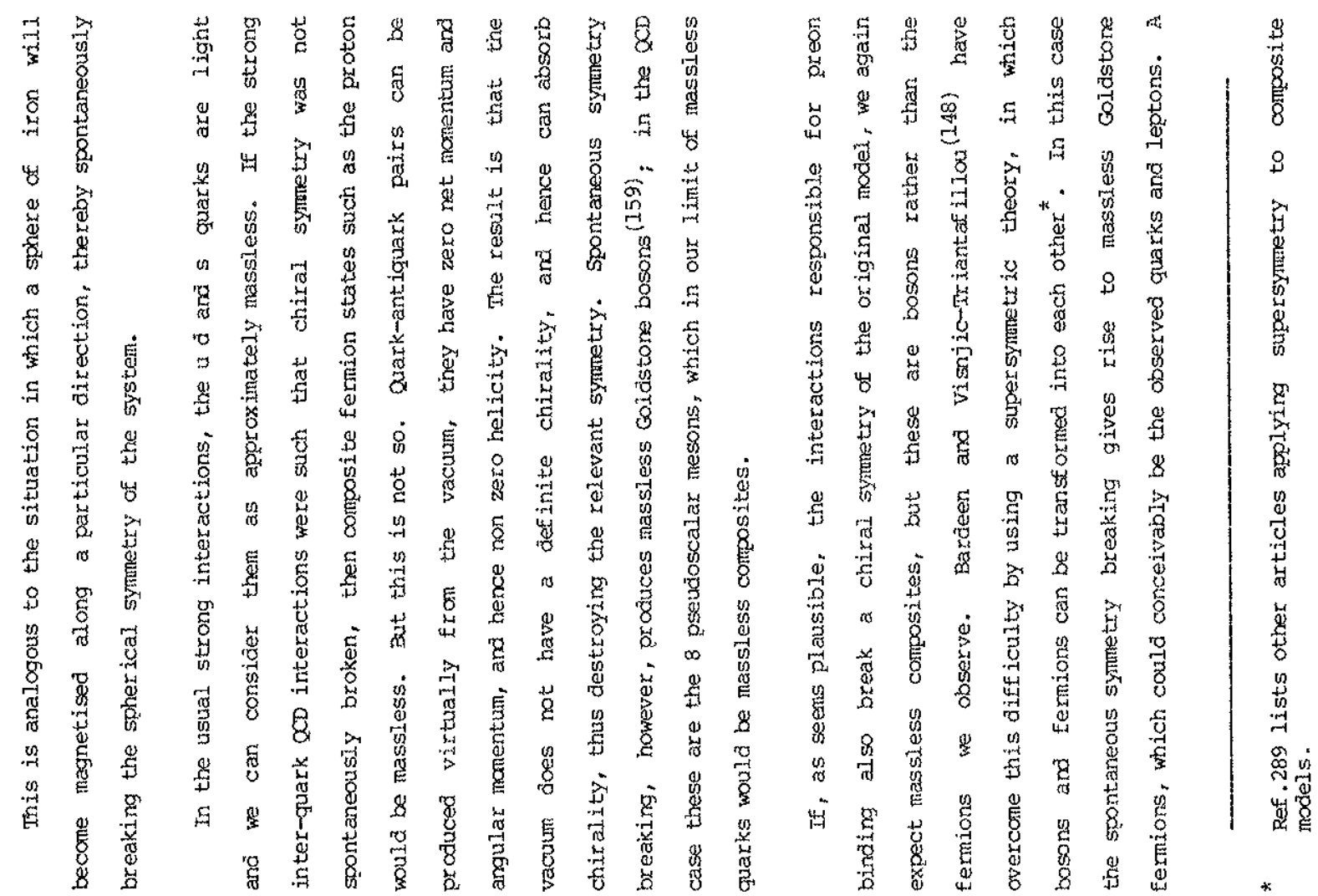




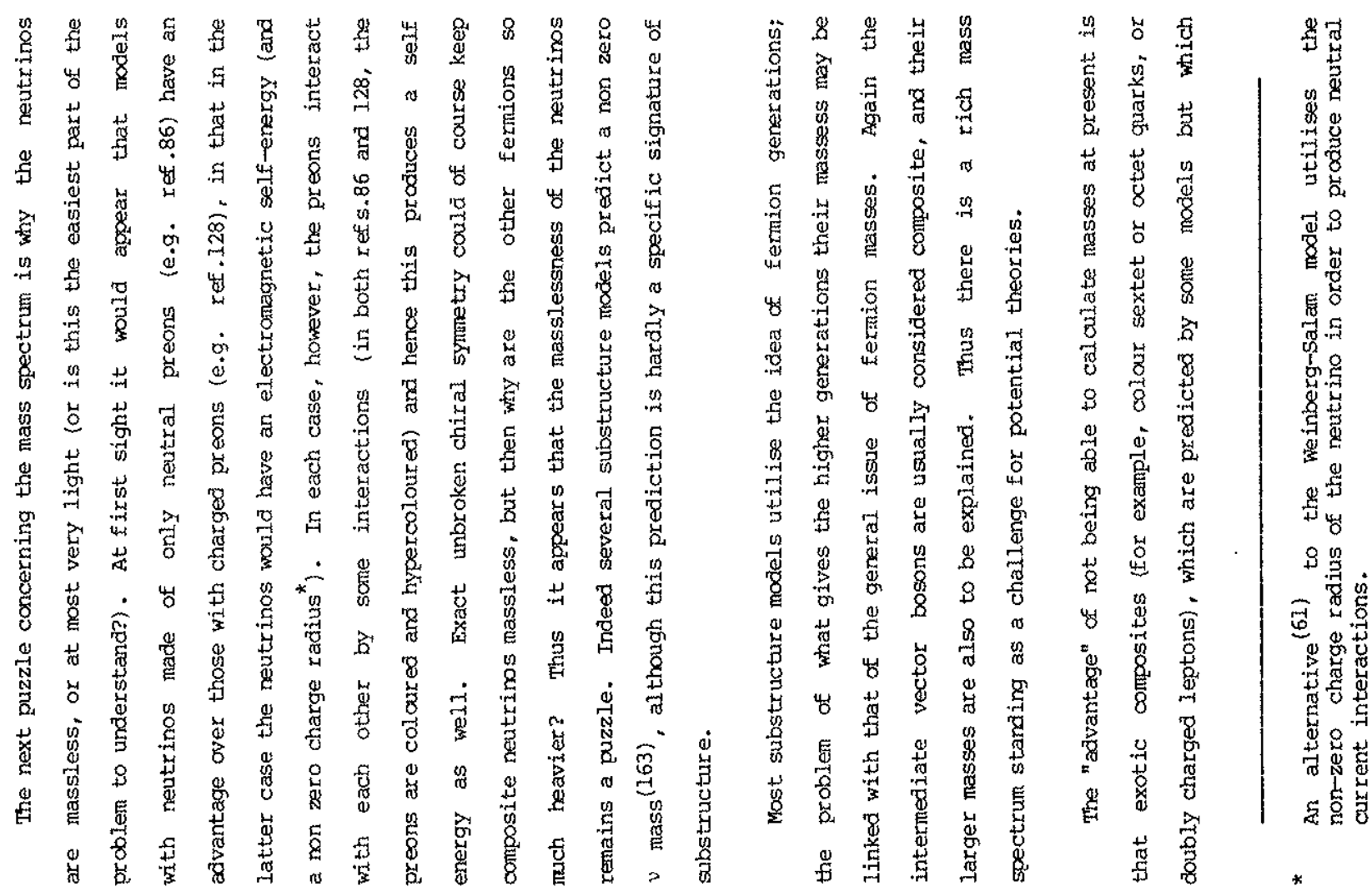

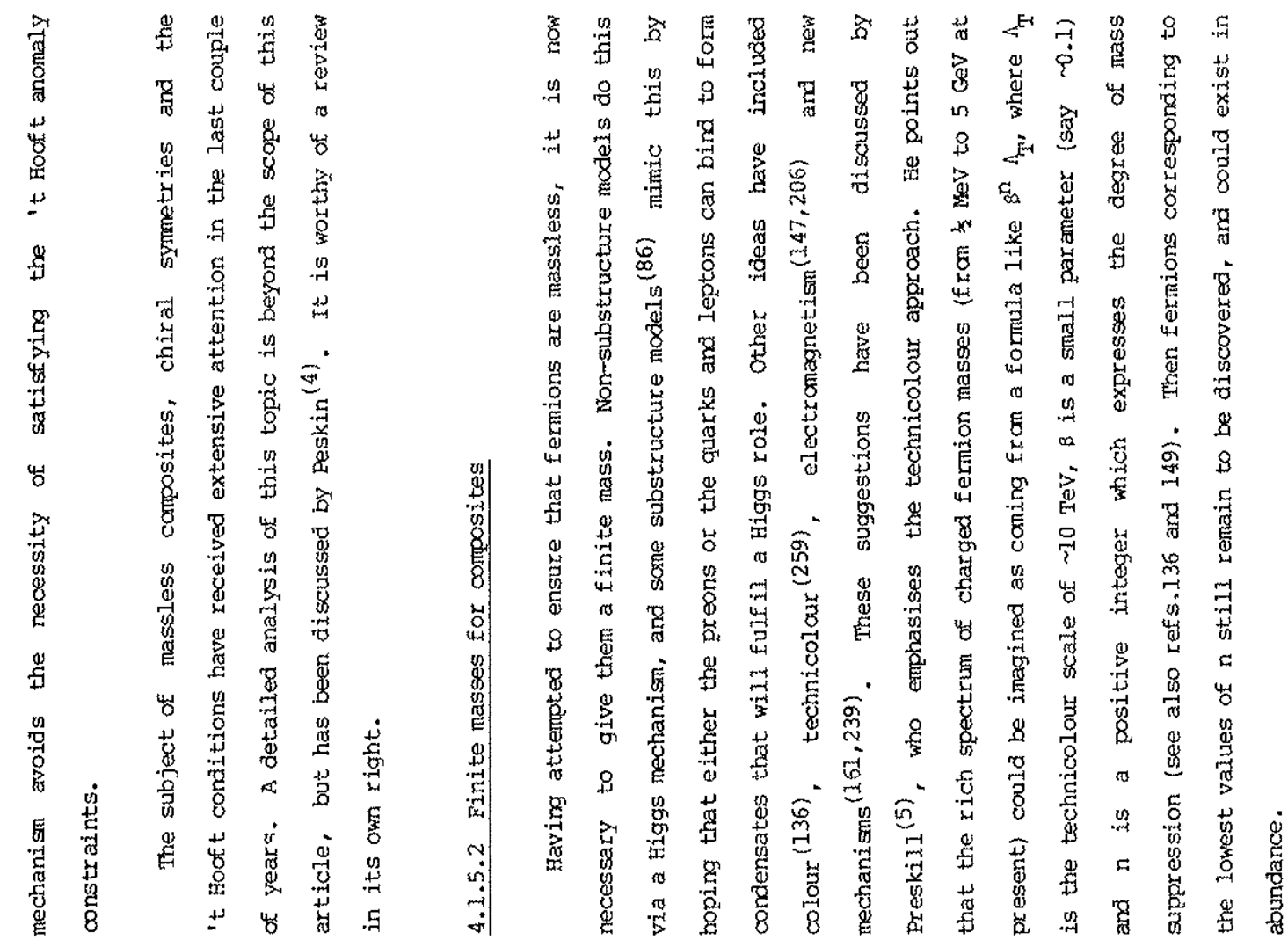



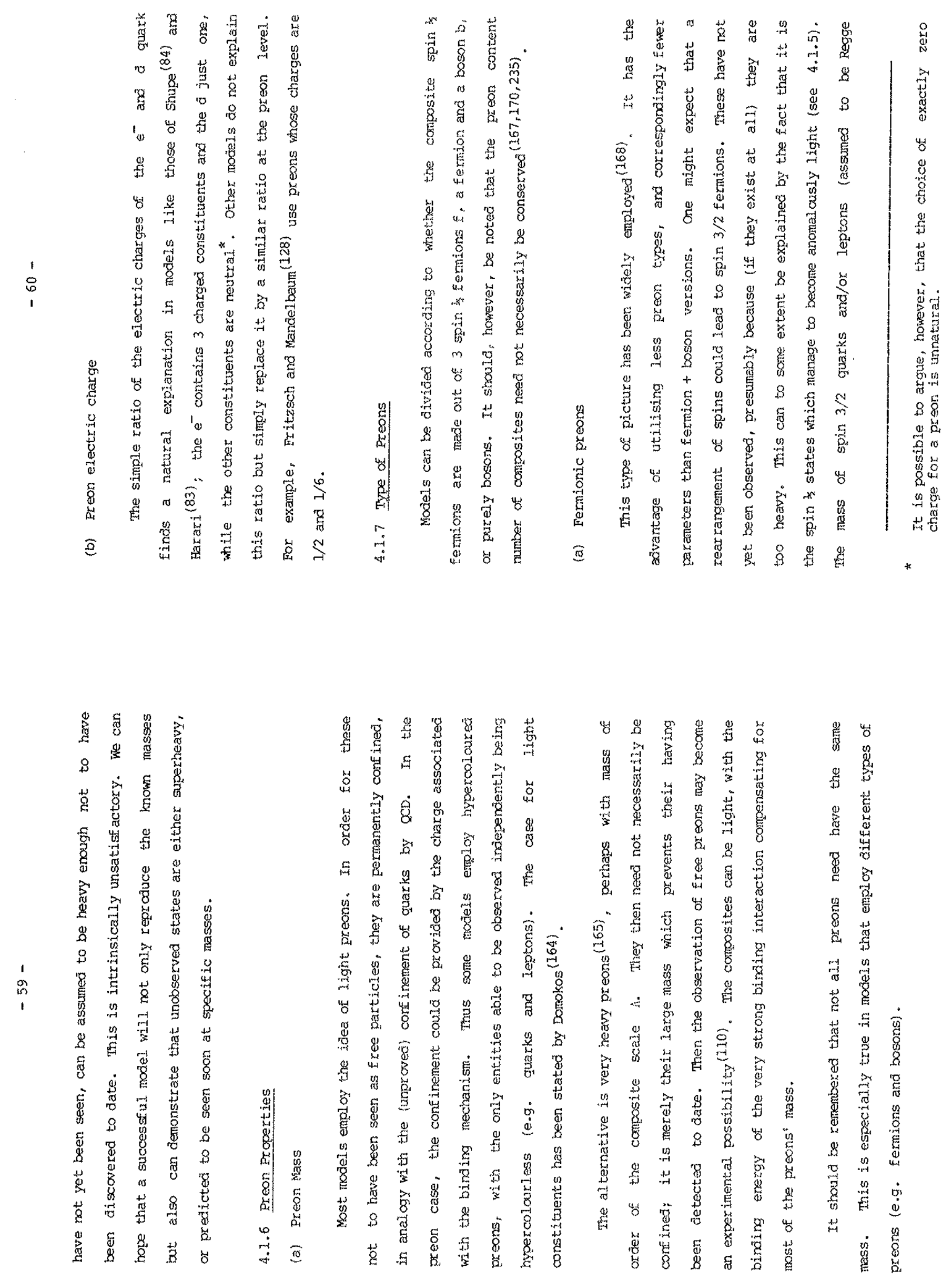

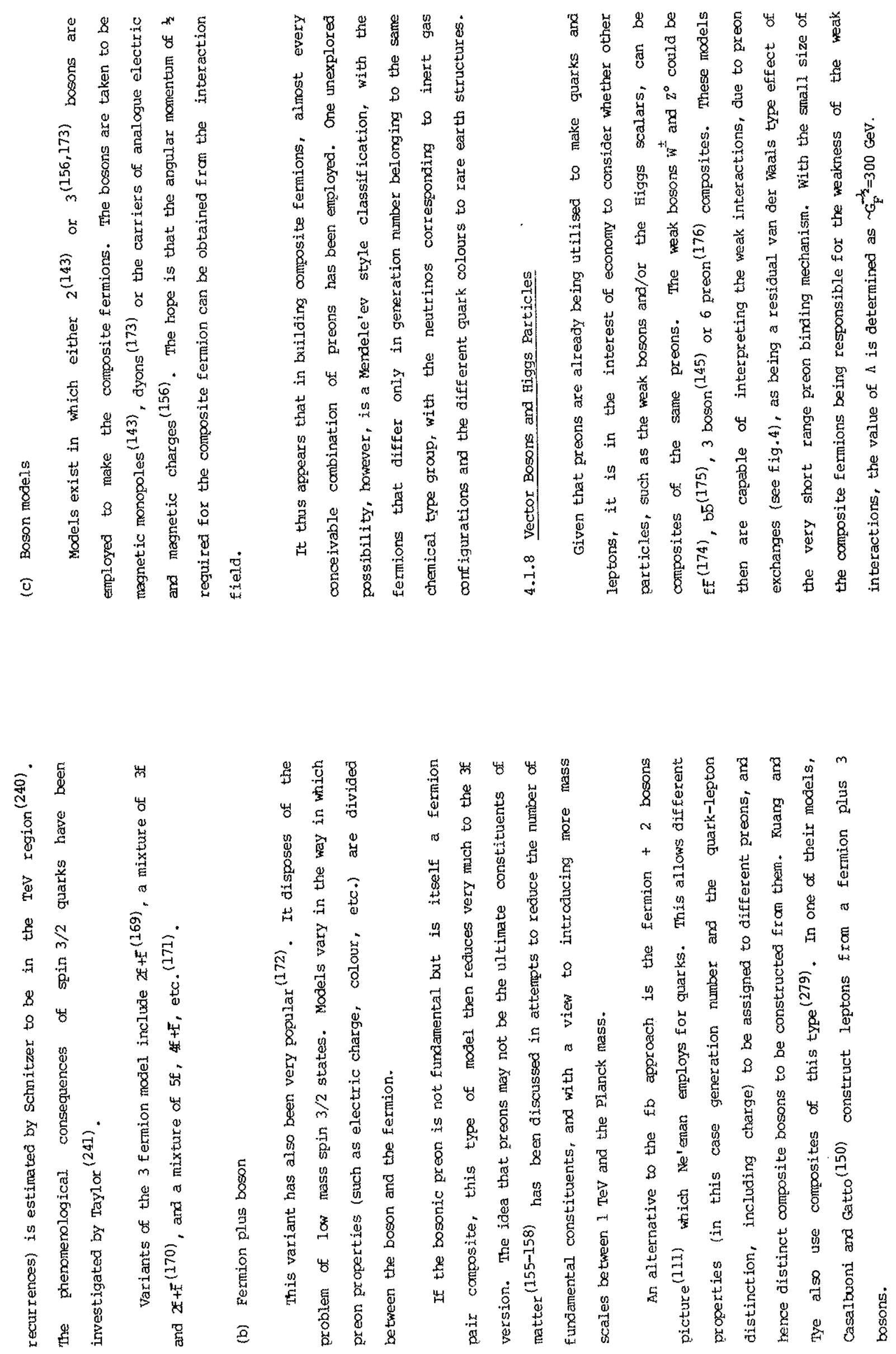


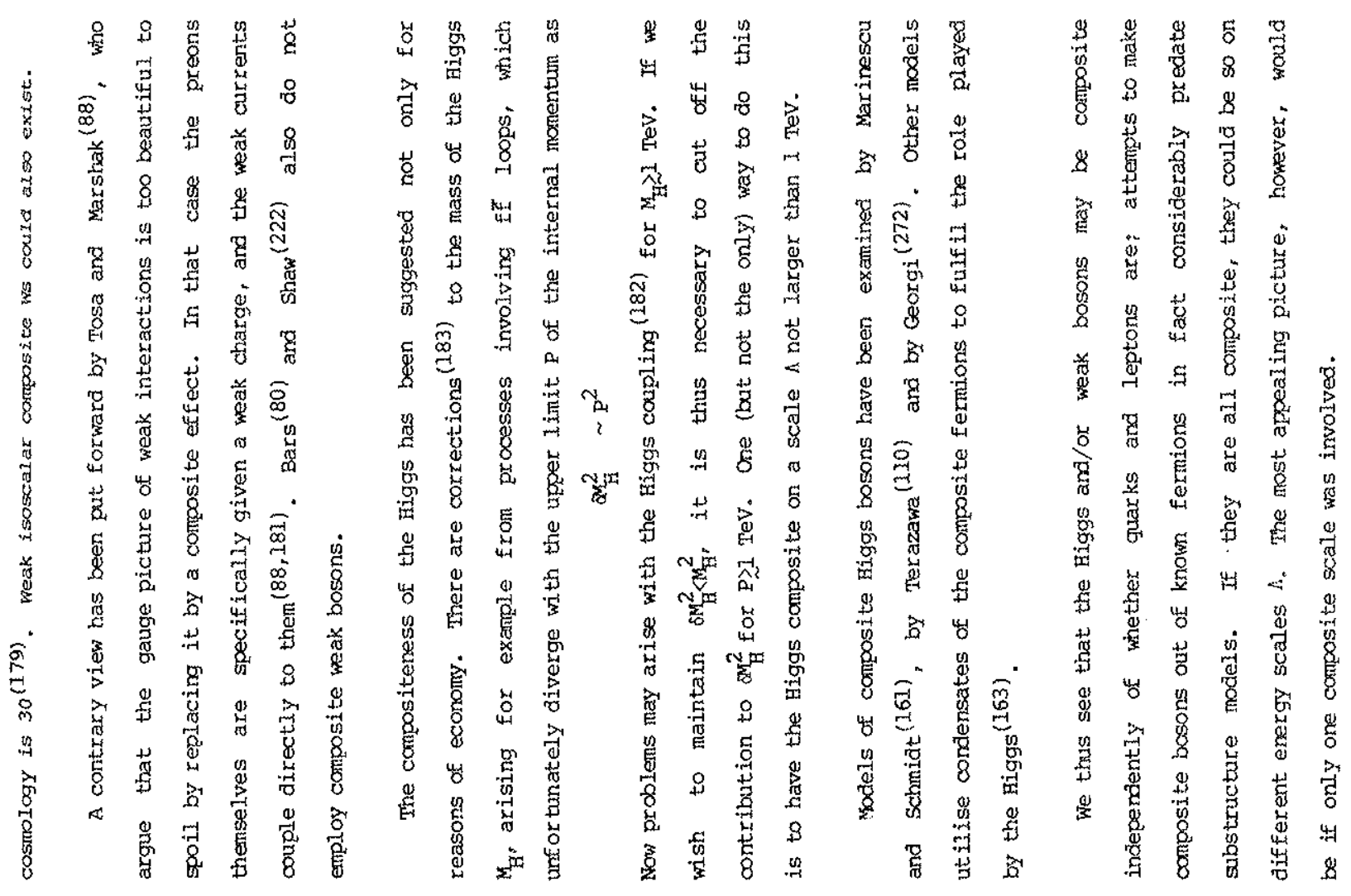

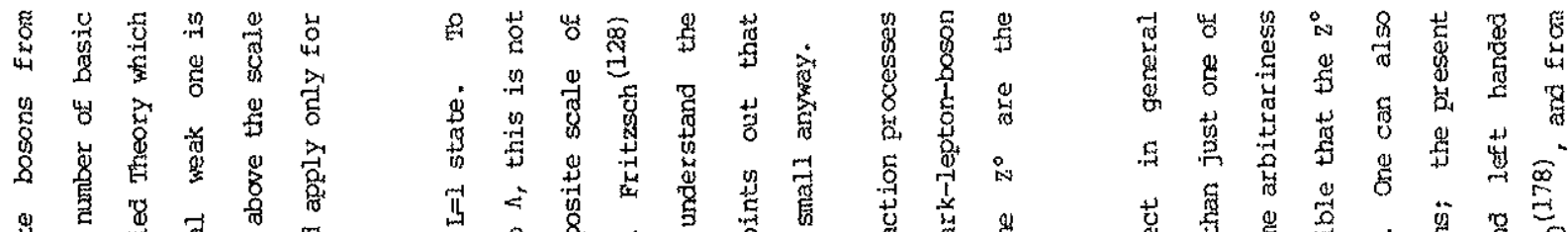

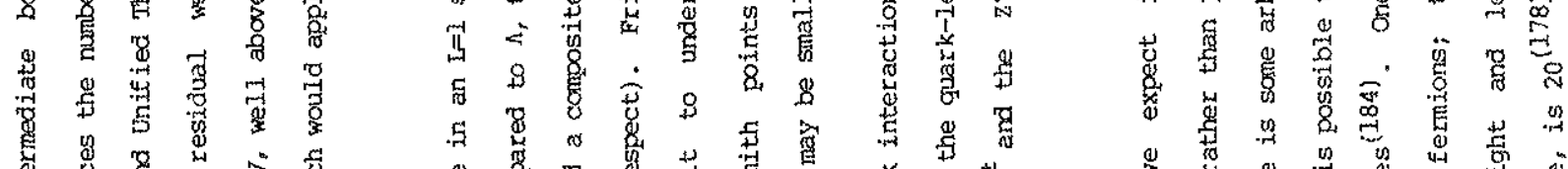

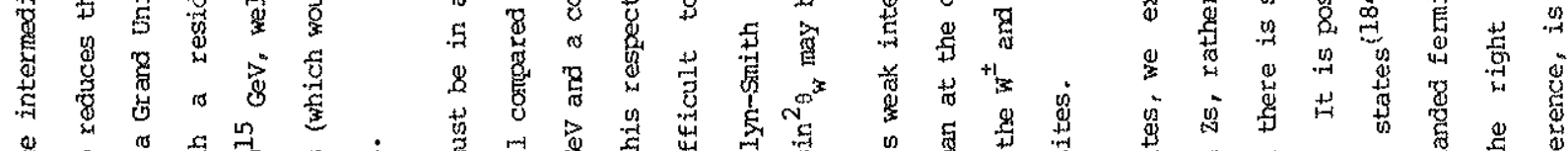

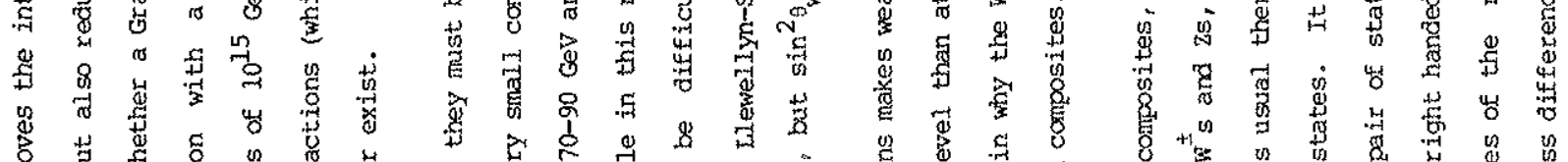

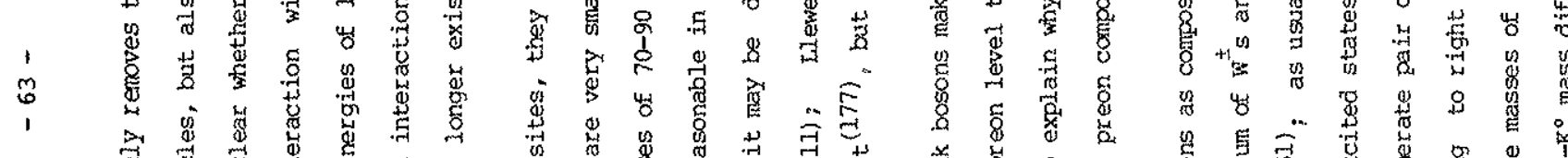

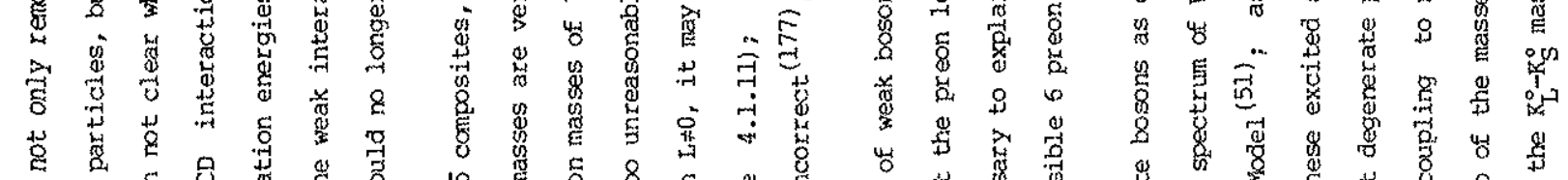

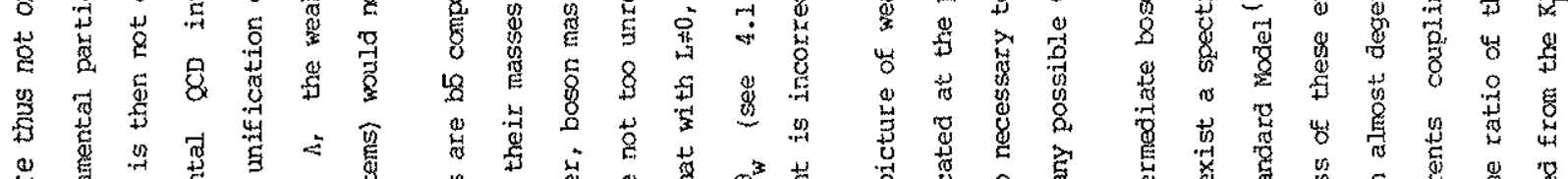

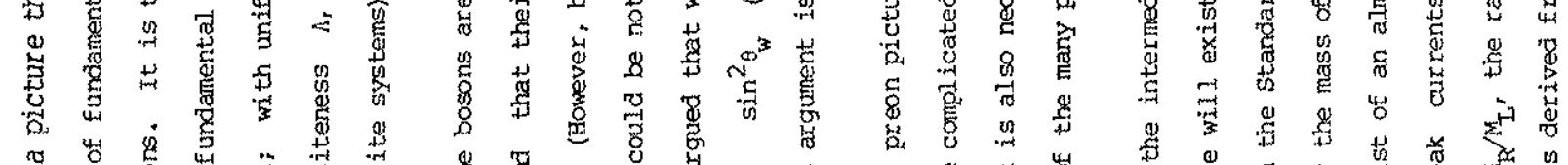

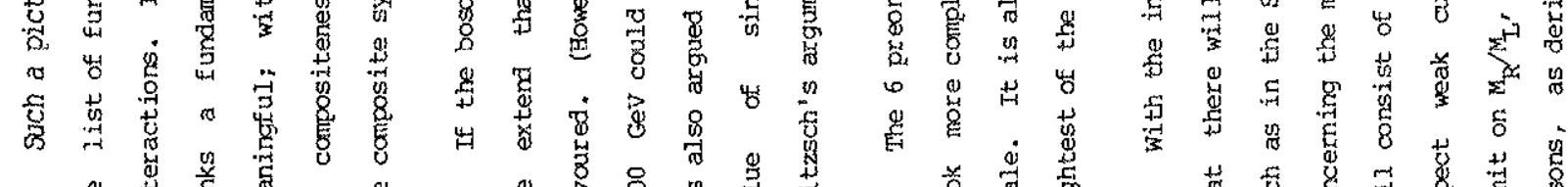

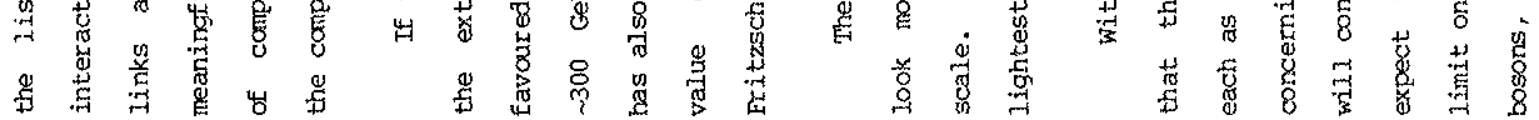



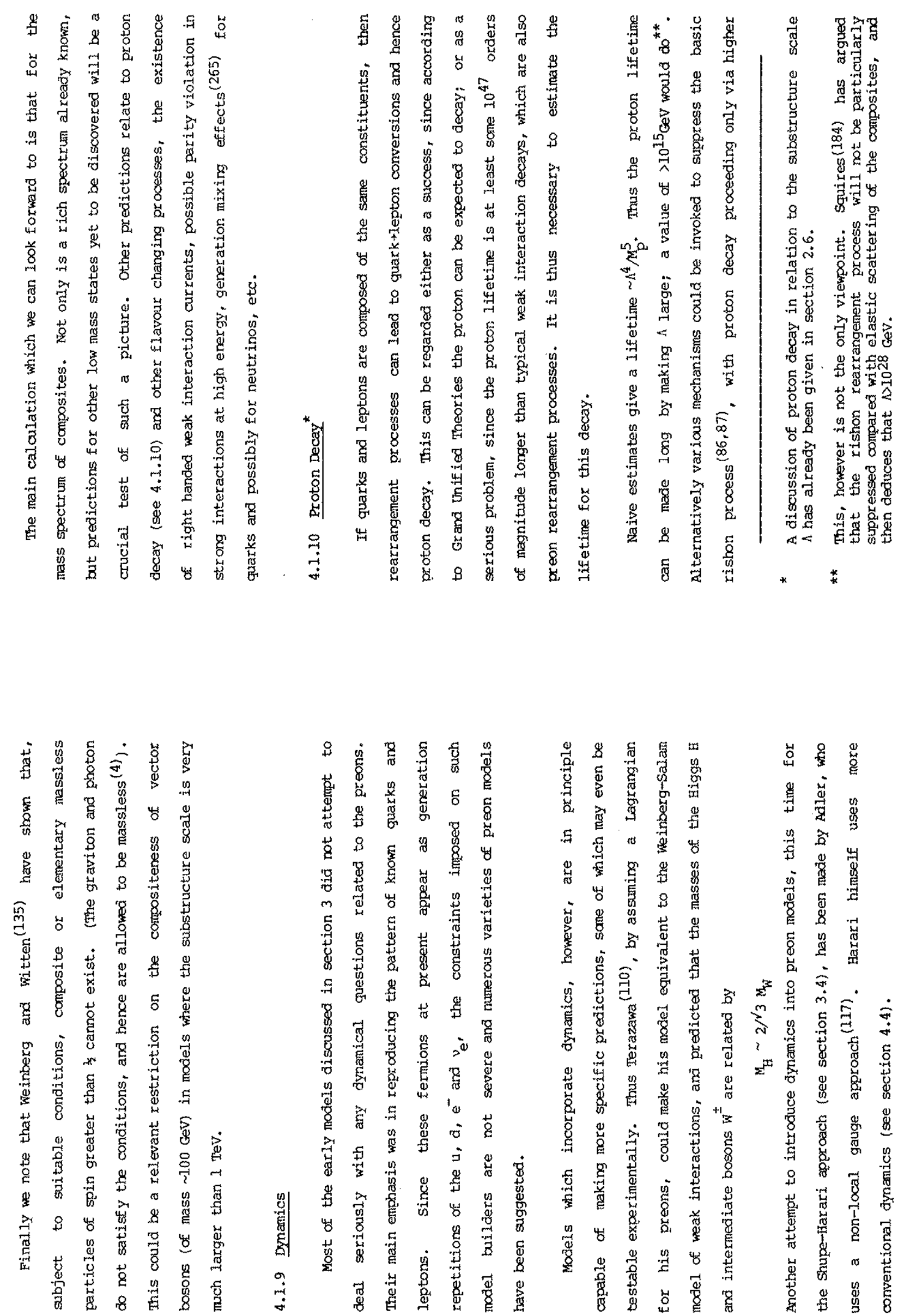

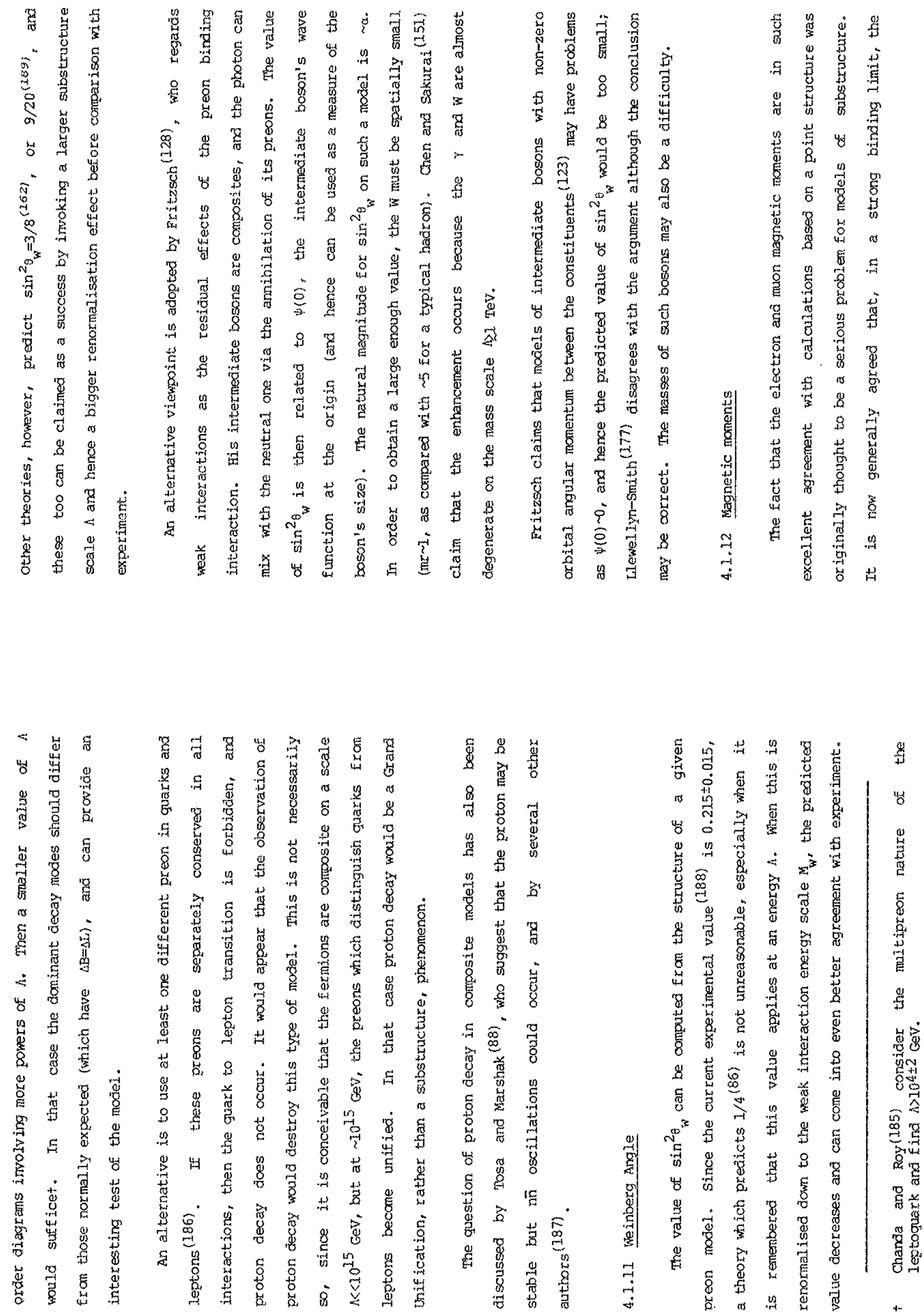


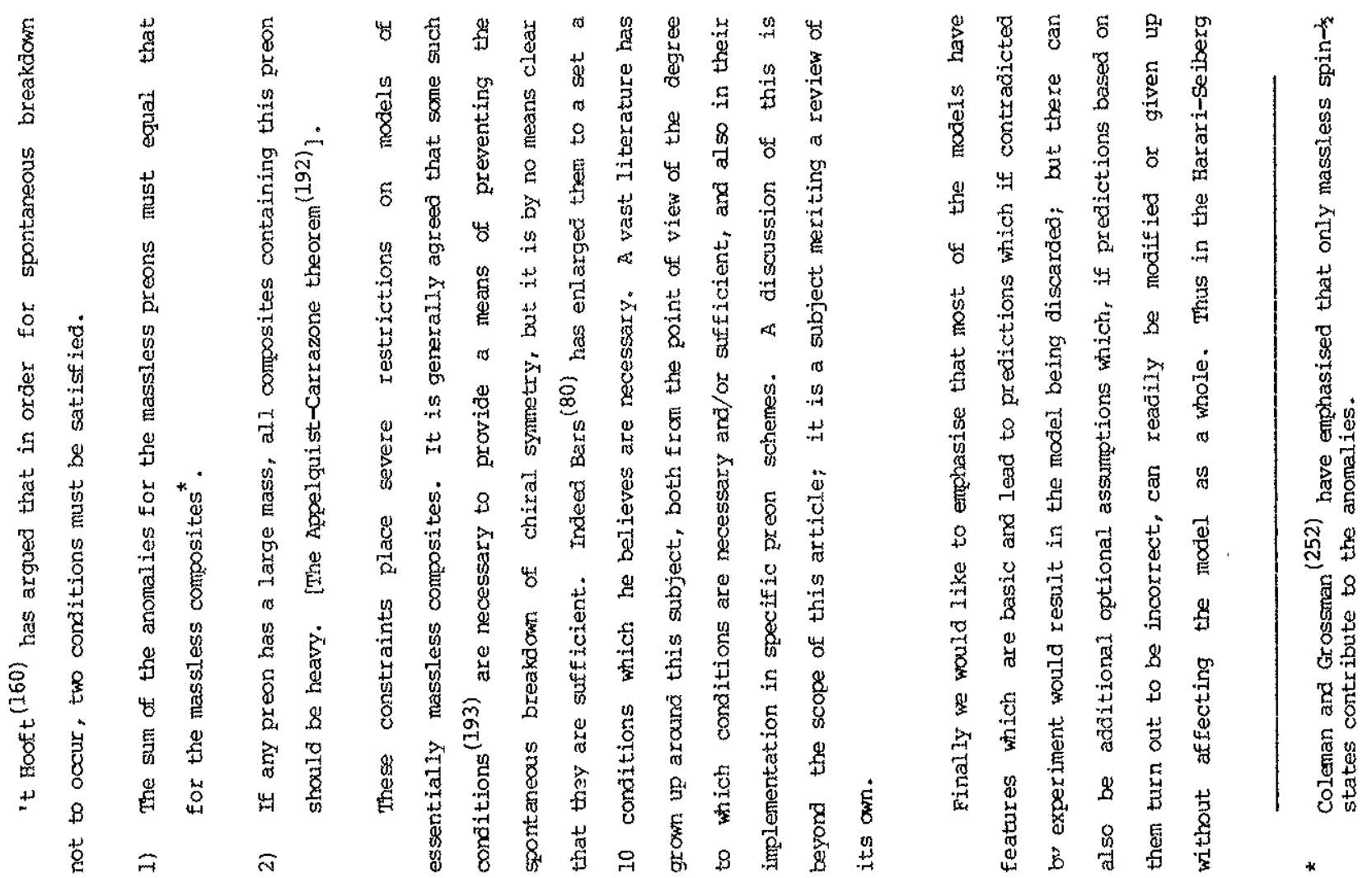

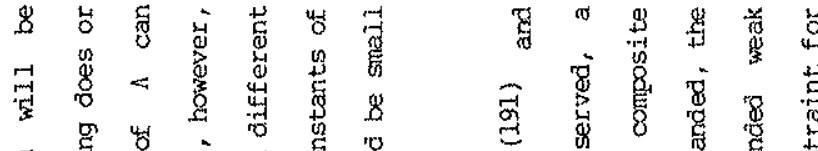

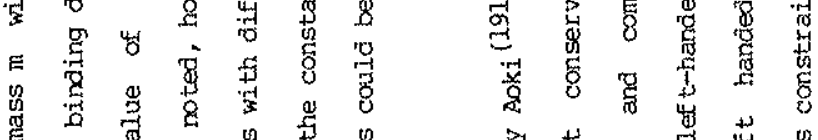

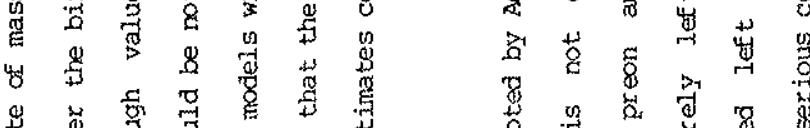

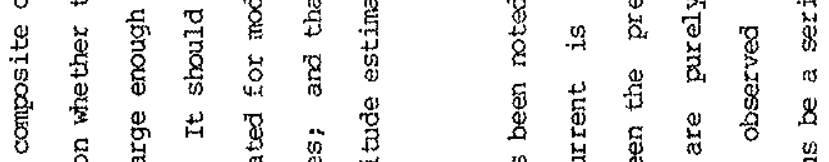

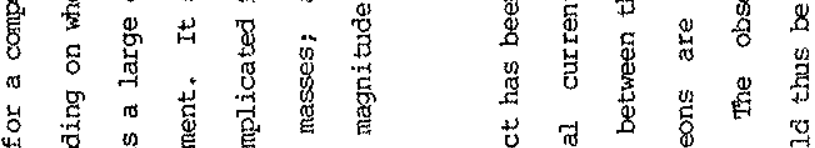

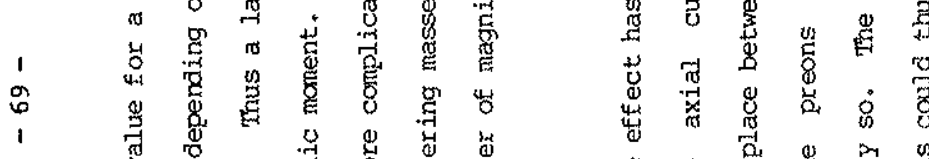

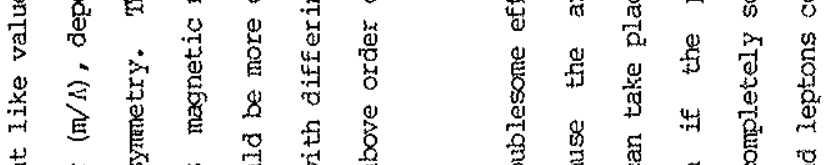

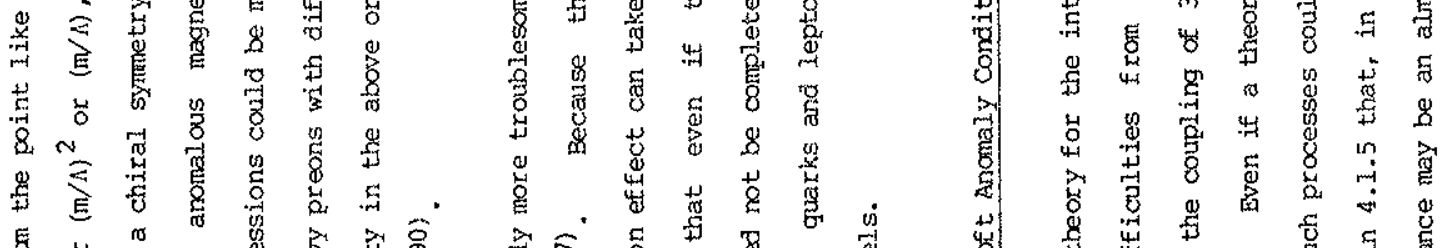

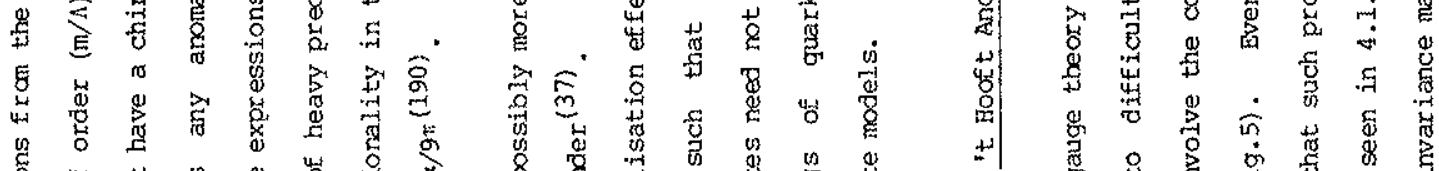

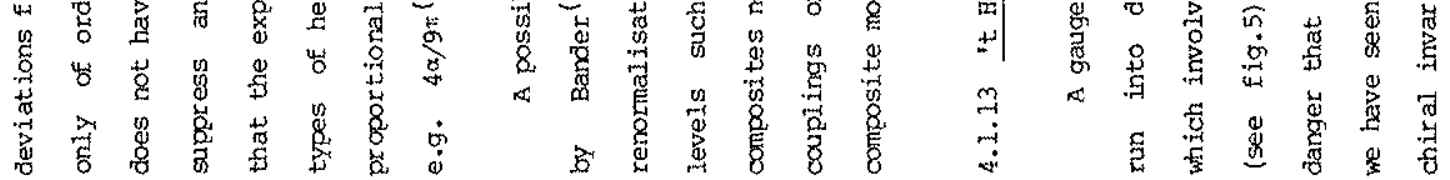




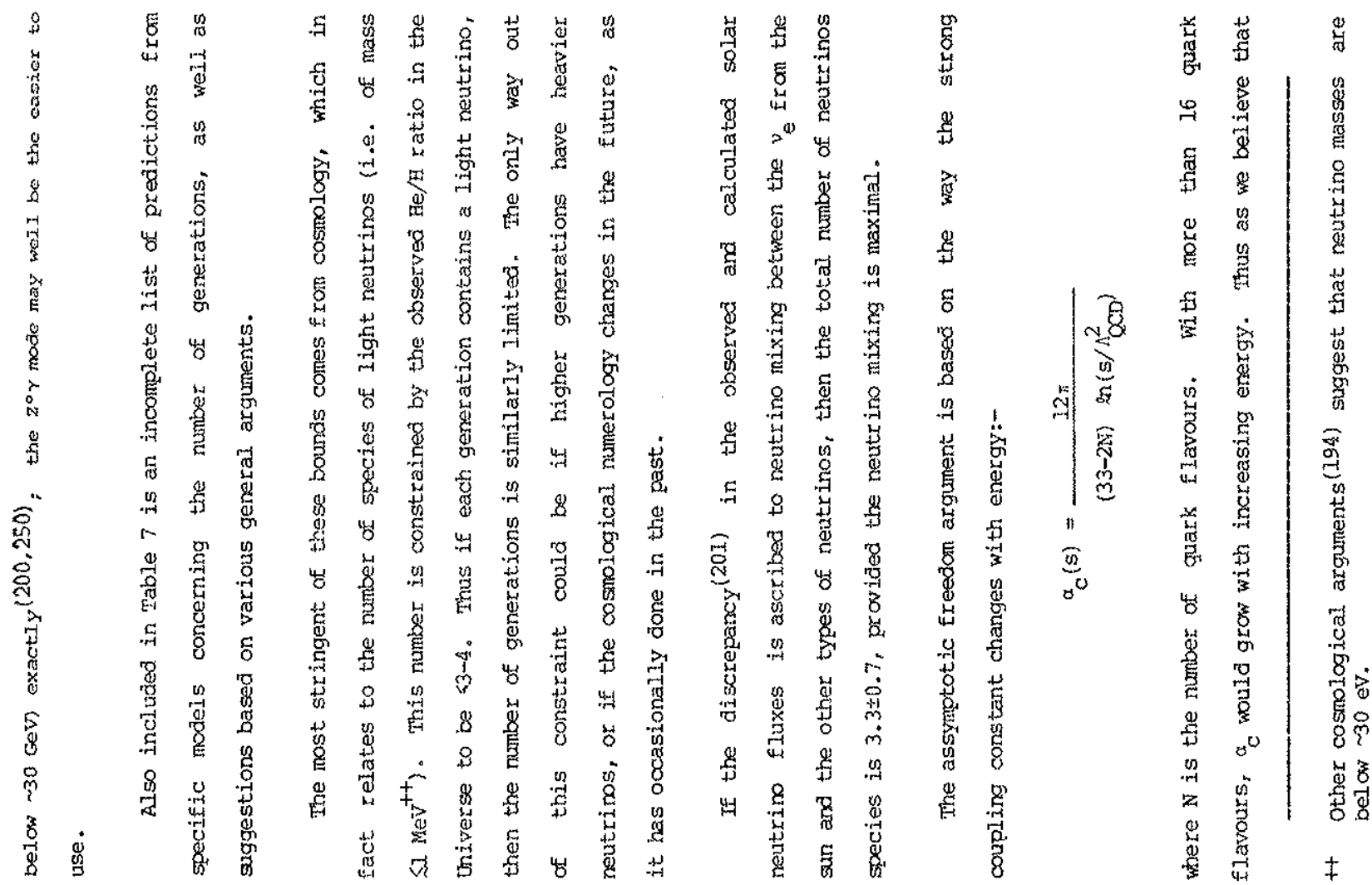

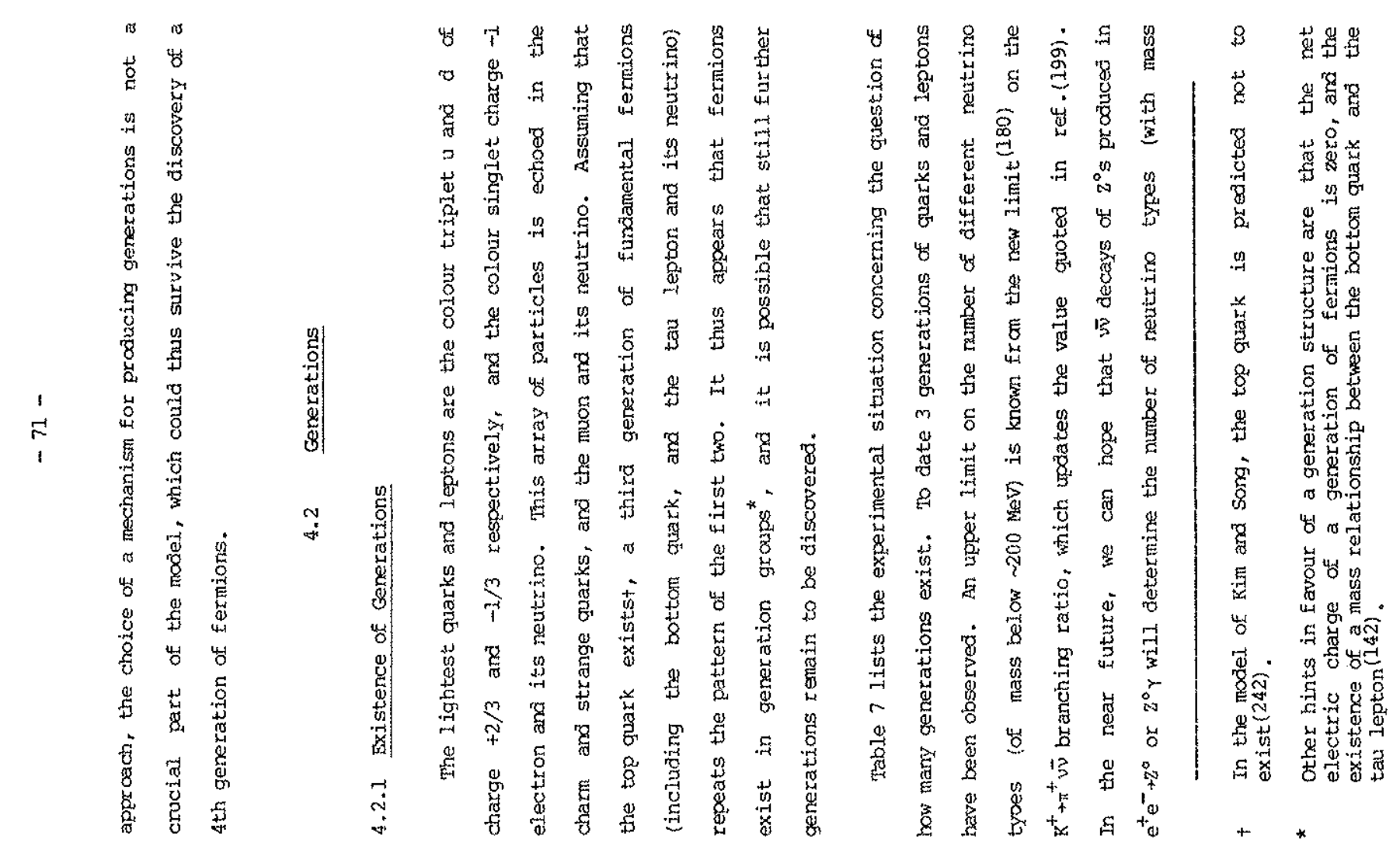



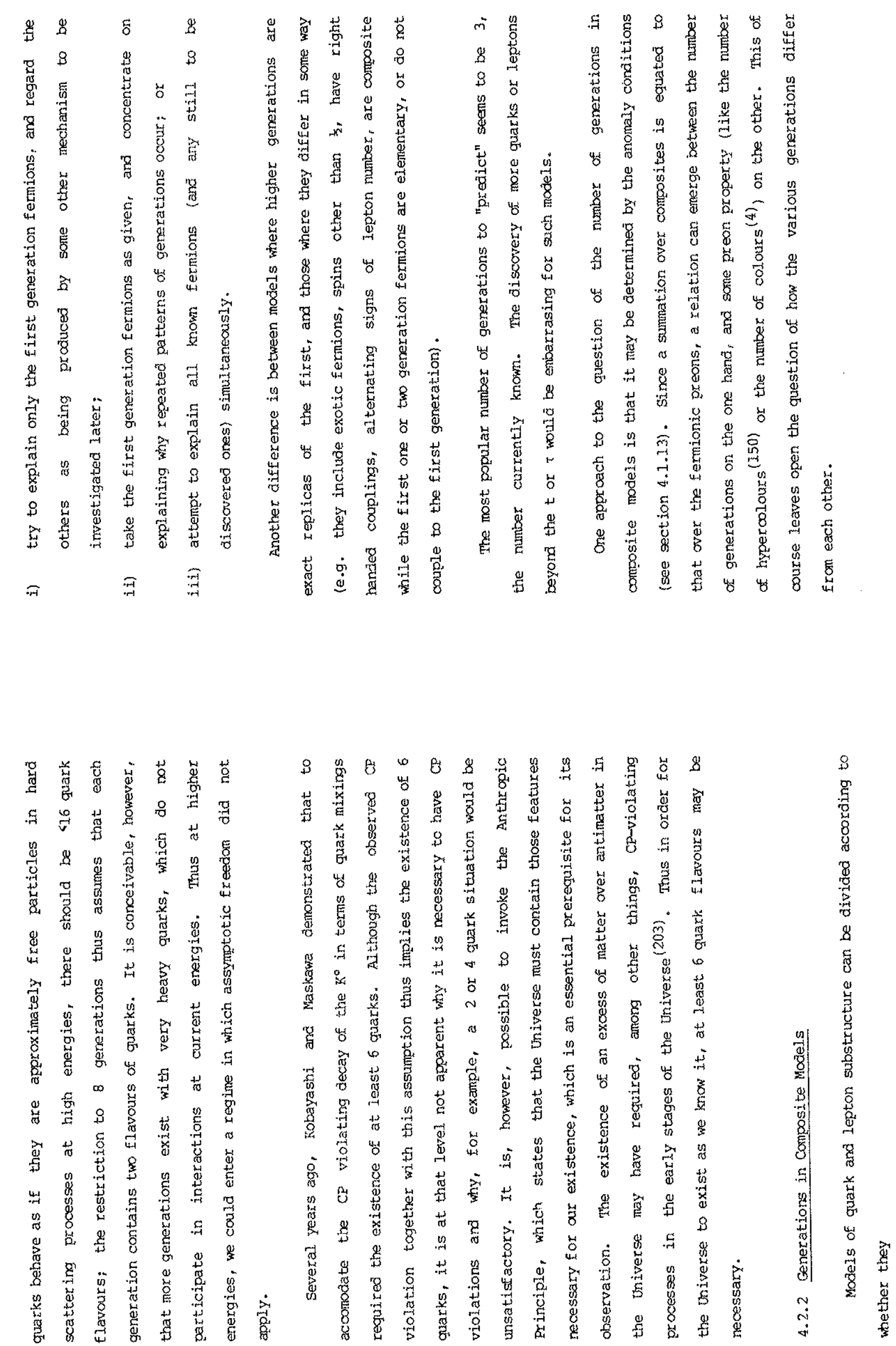

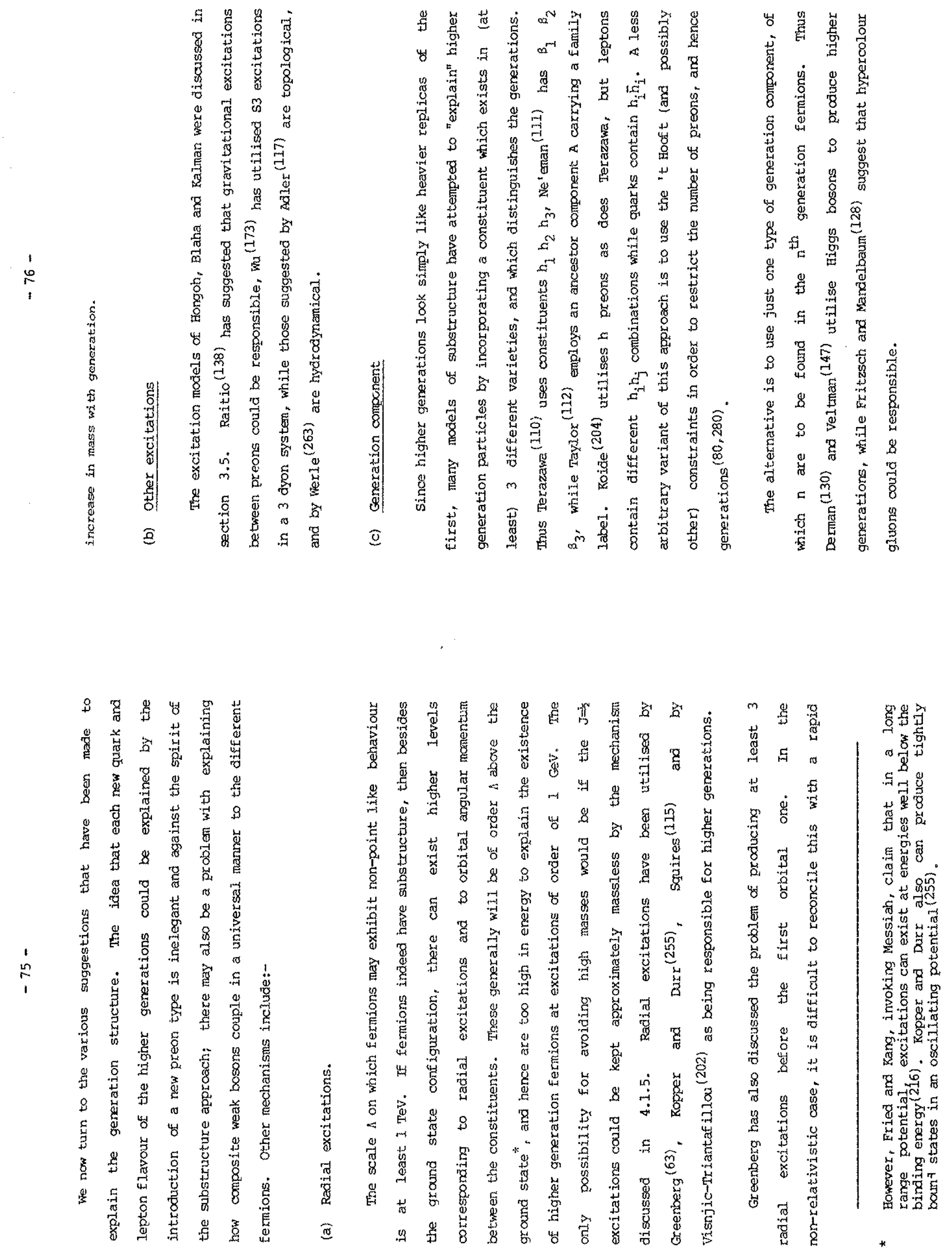

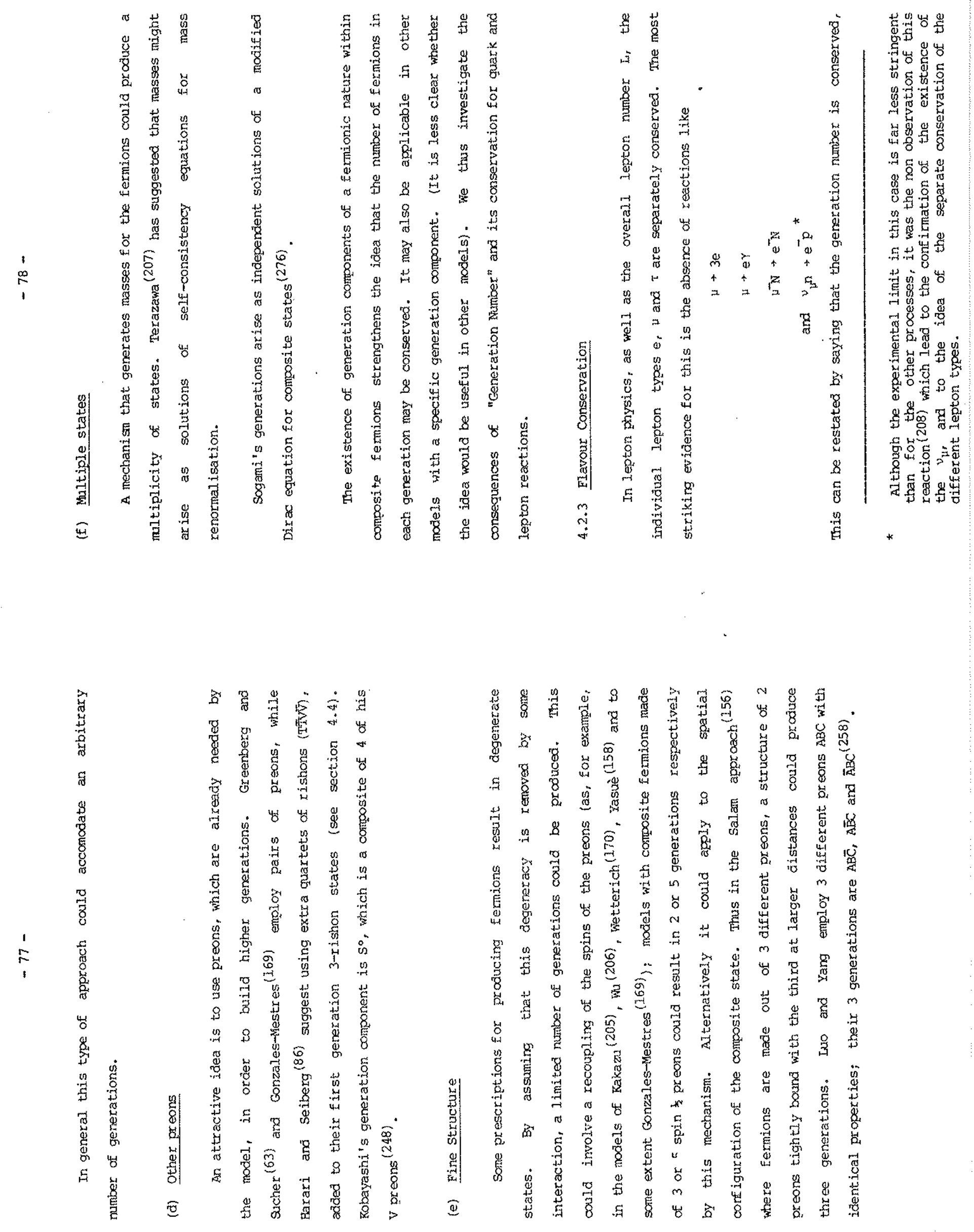

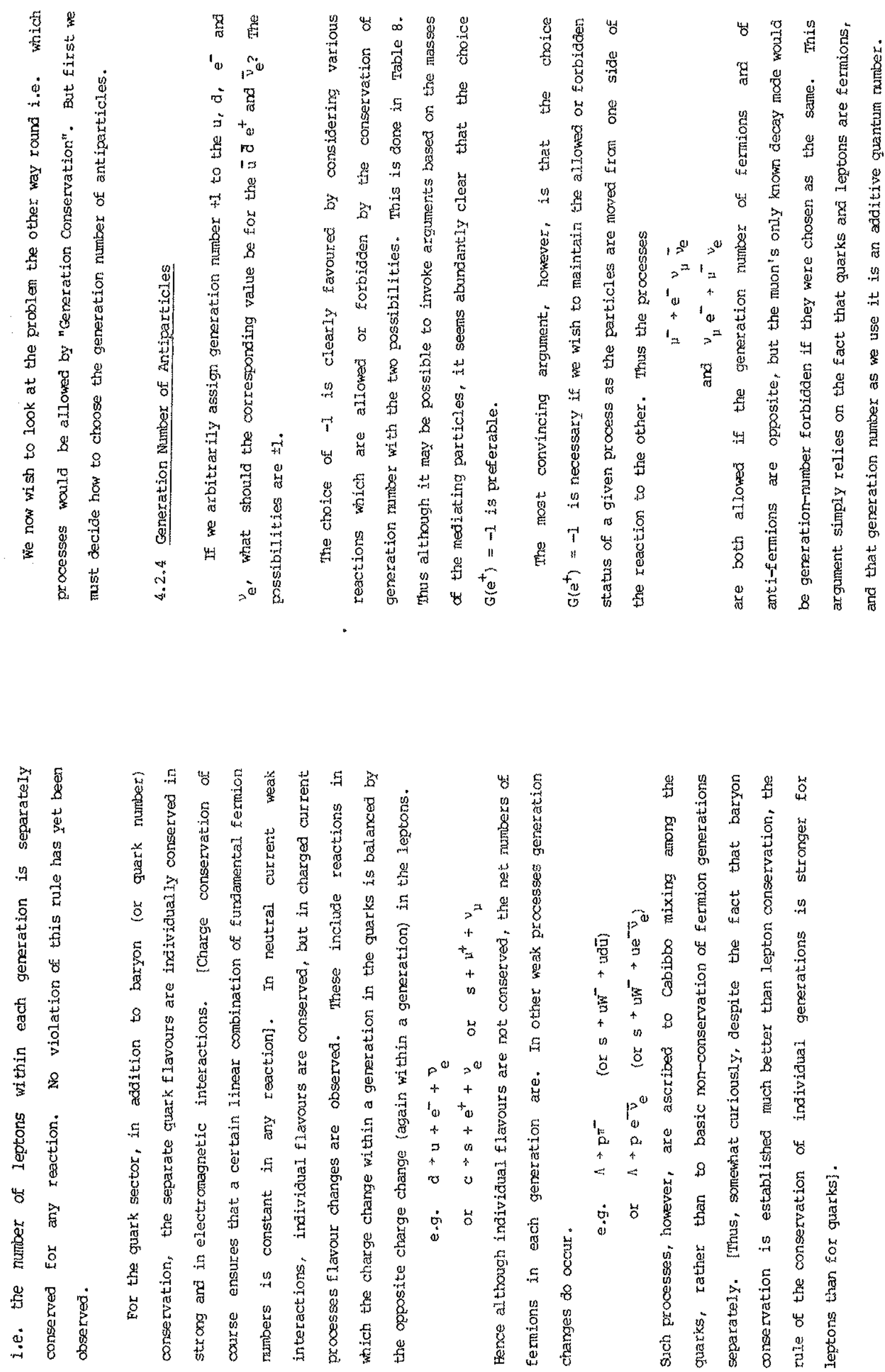


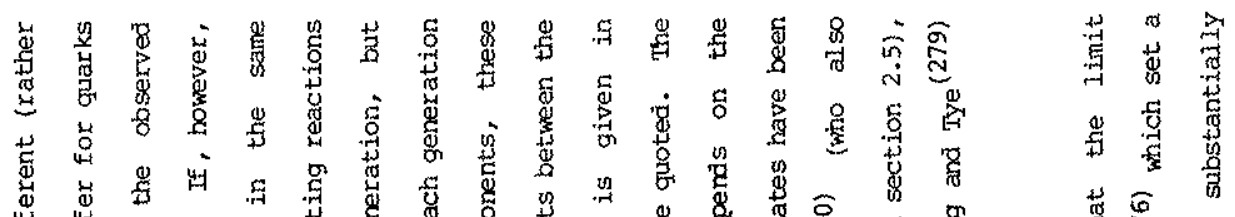

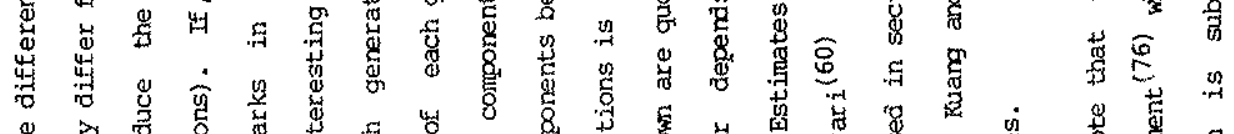

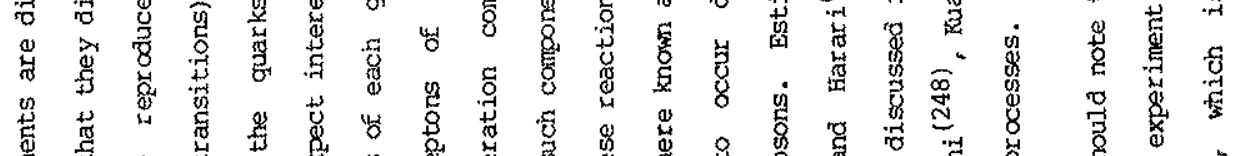

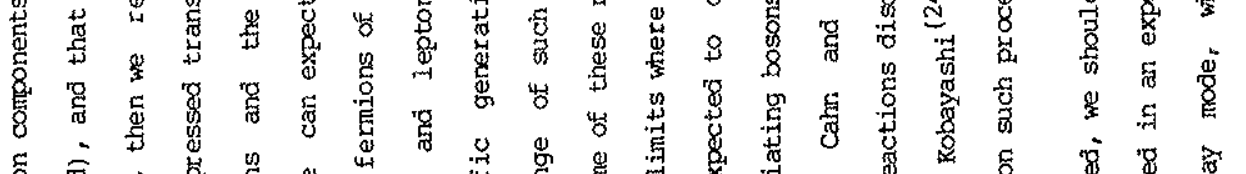

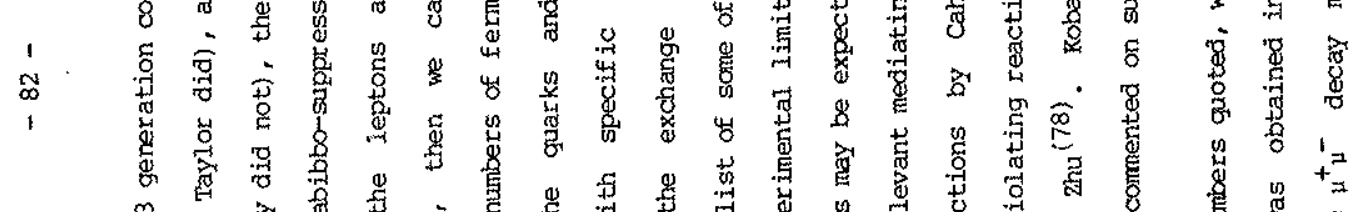

m

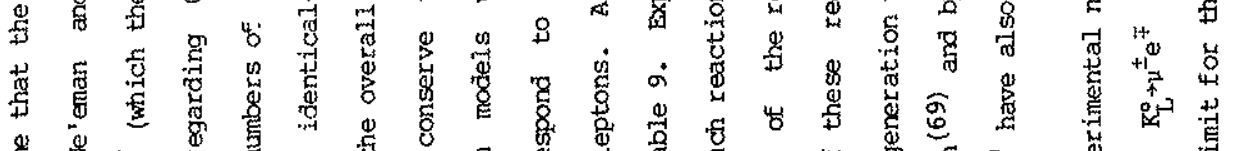

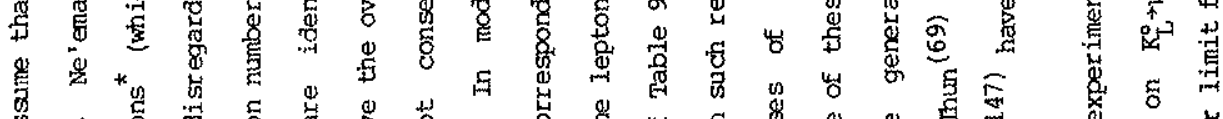

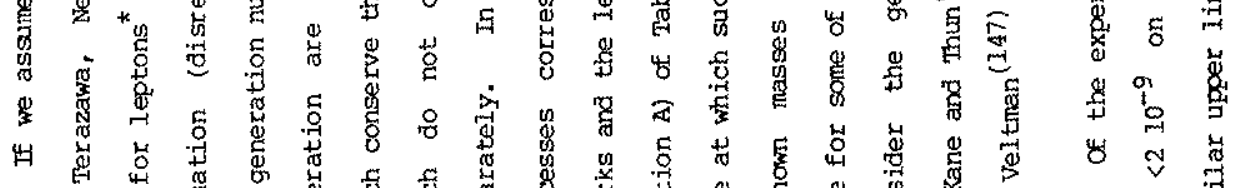

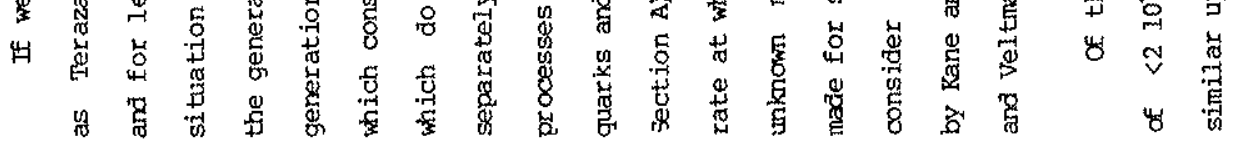
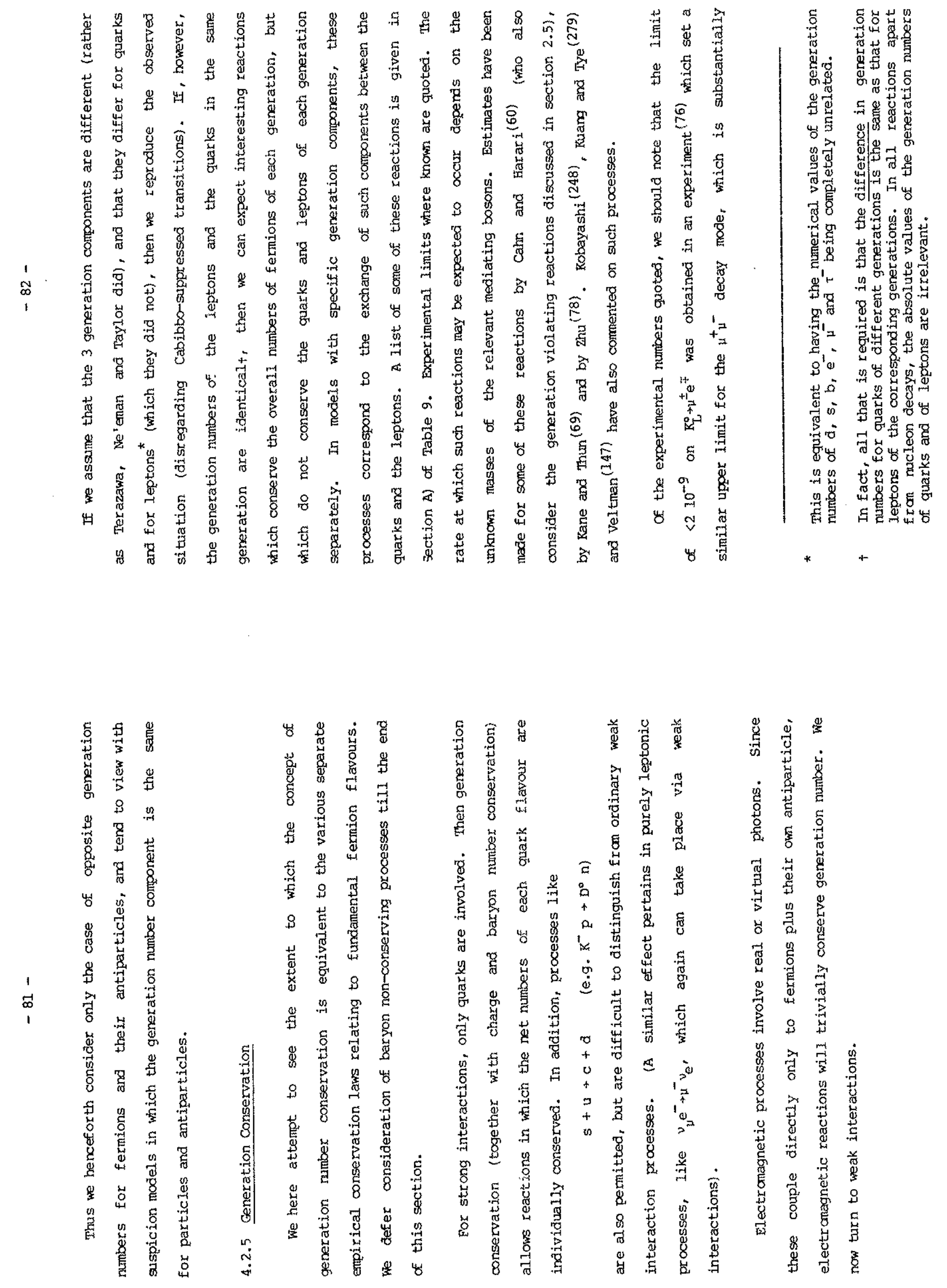

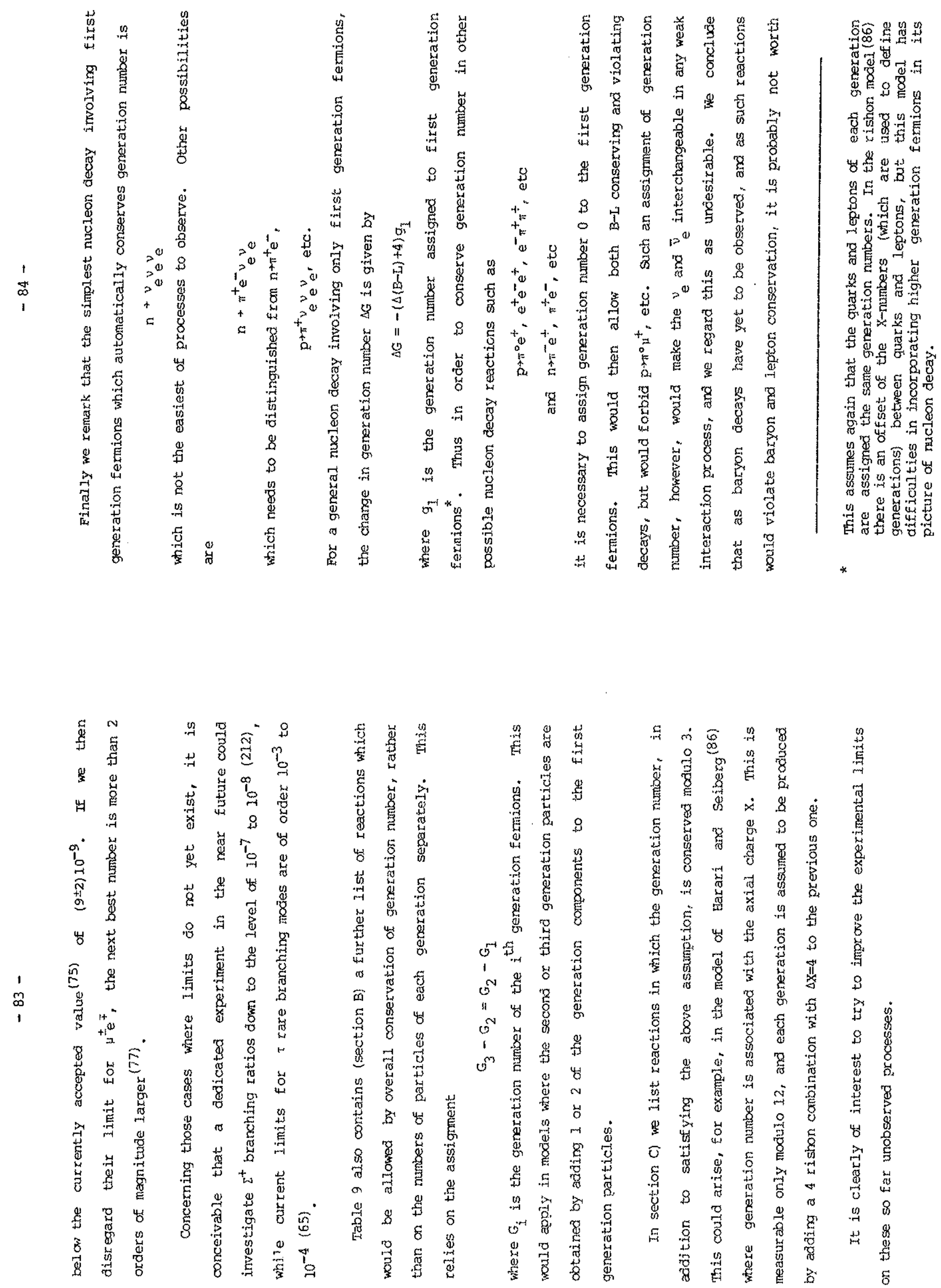

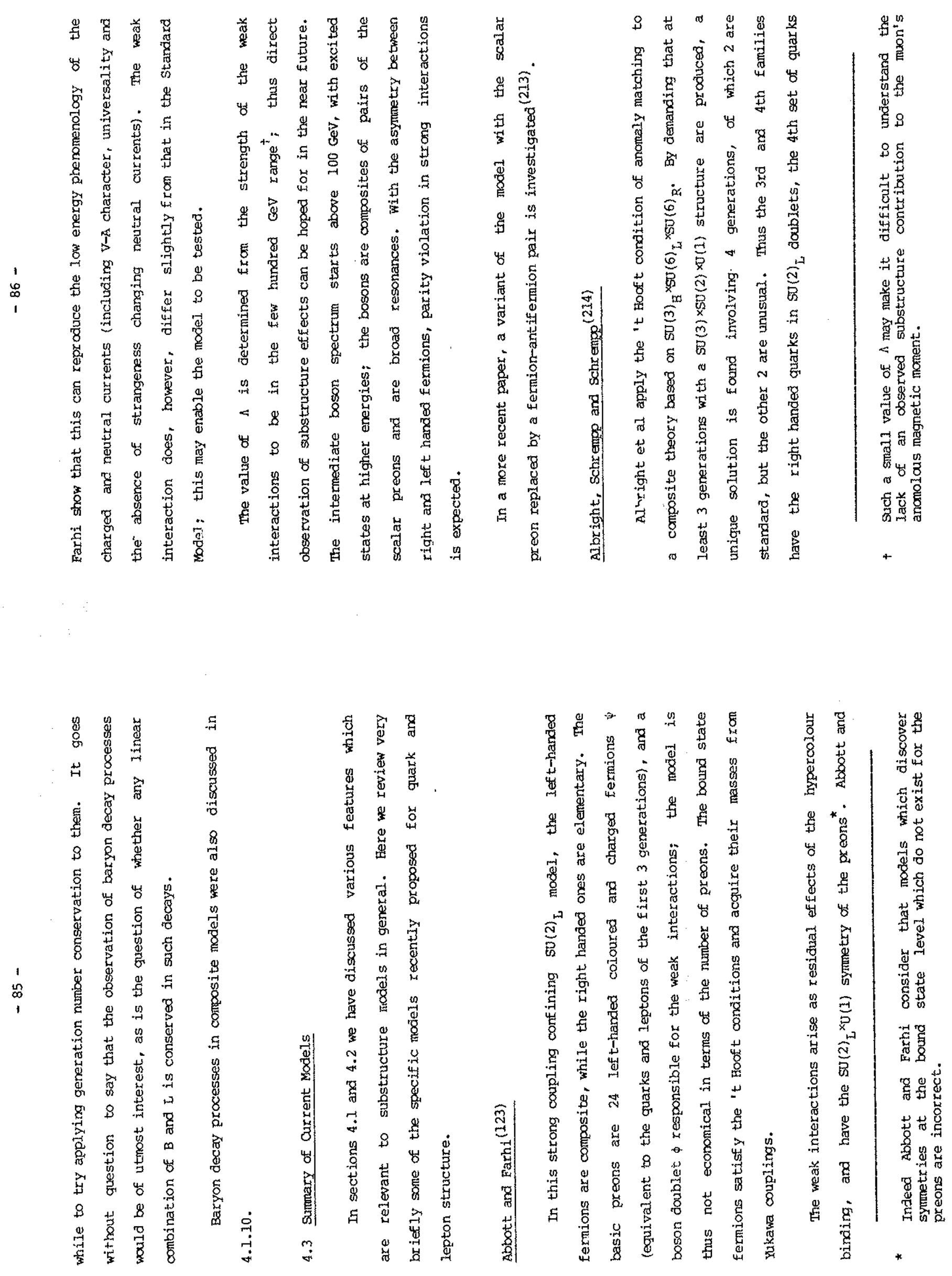

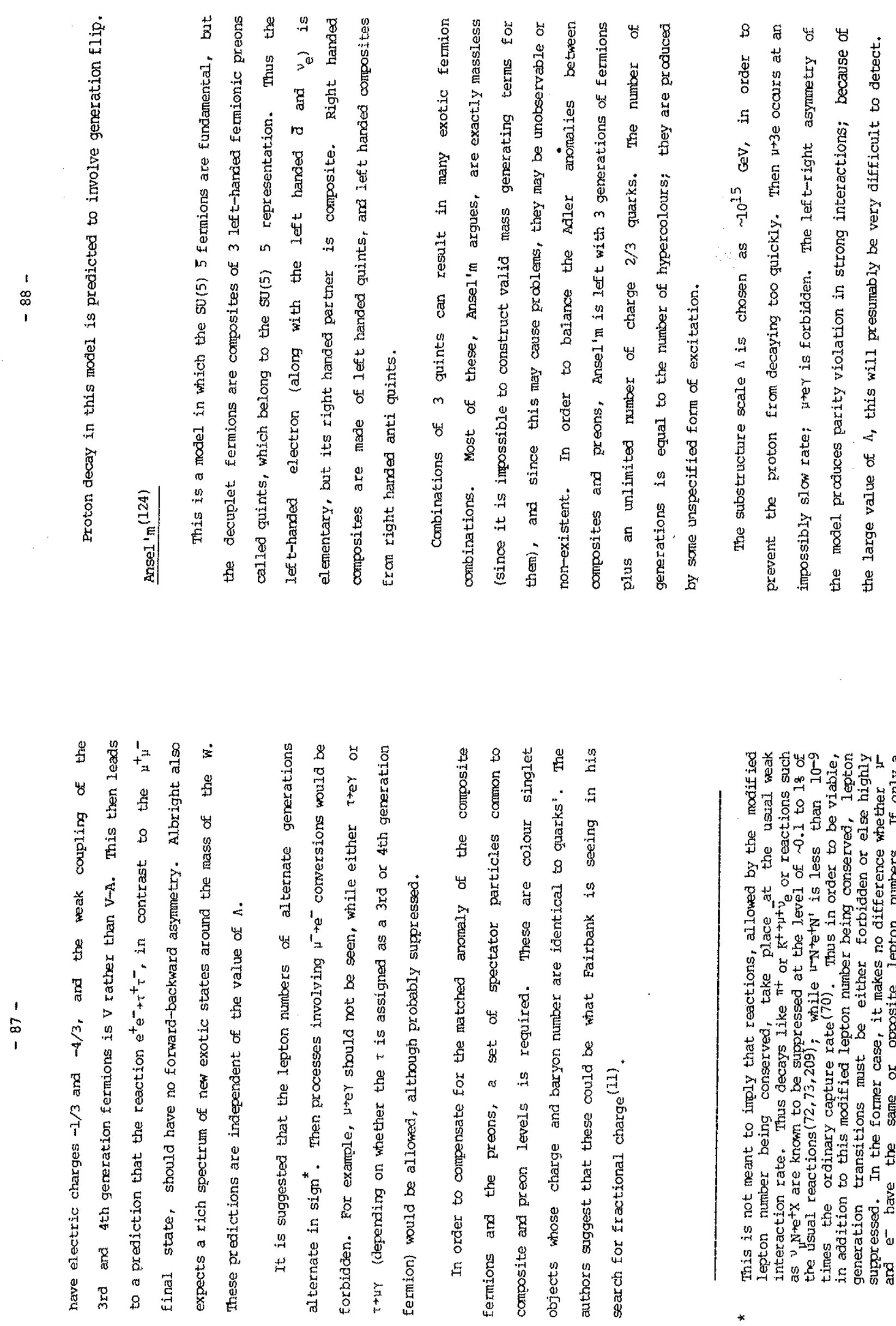

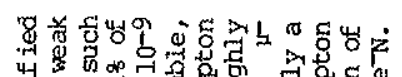

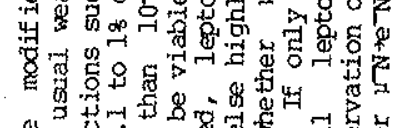

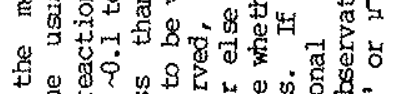
\$

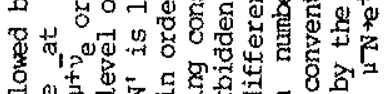

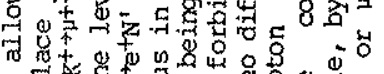
等

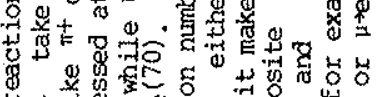

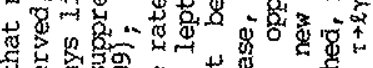
F

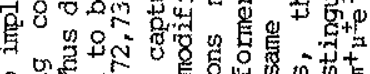

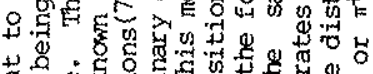
淢

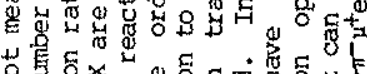

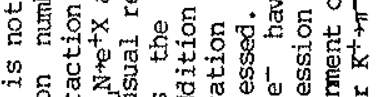

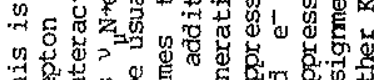

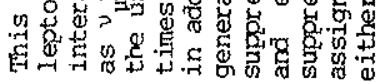



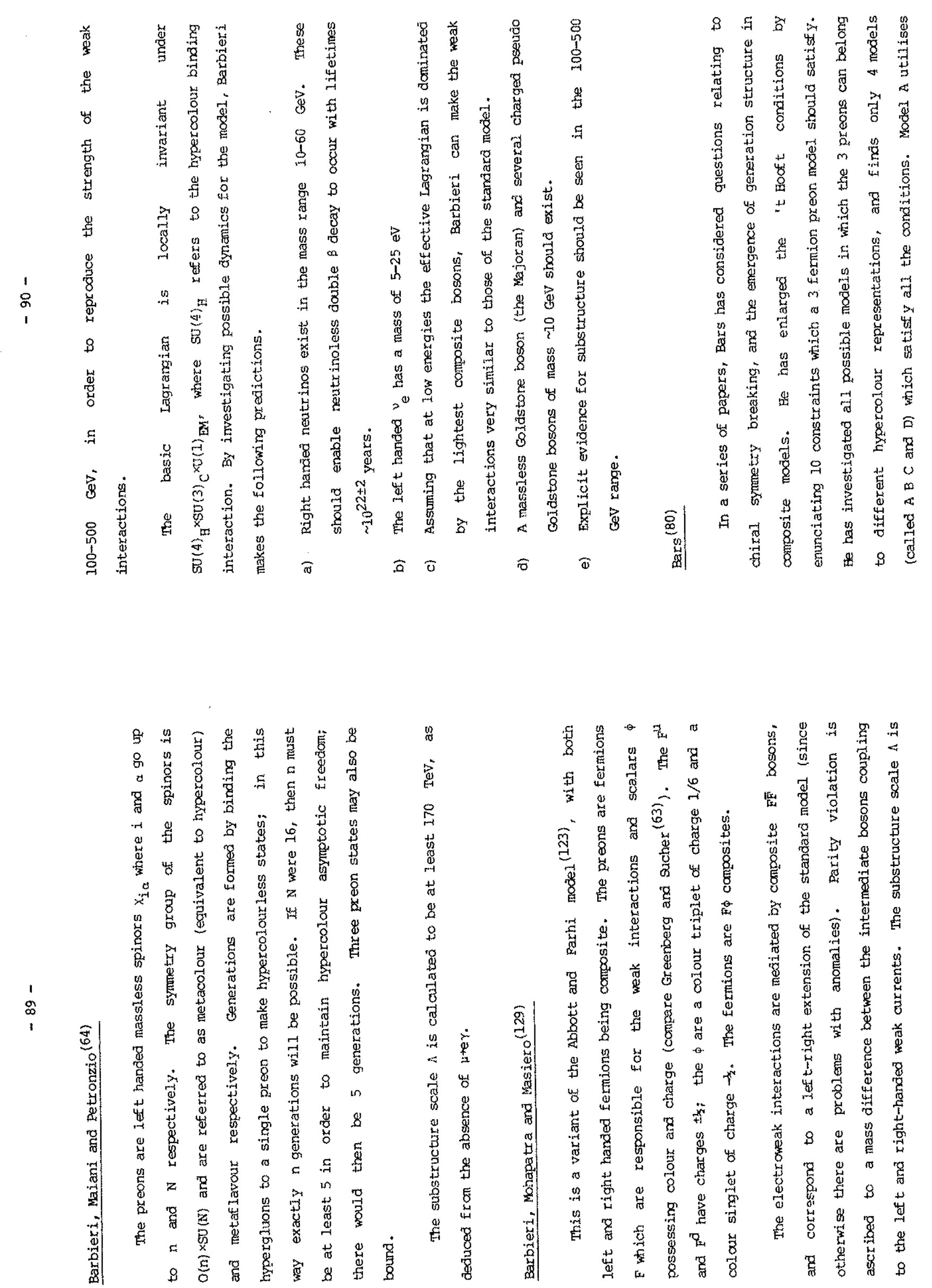


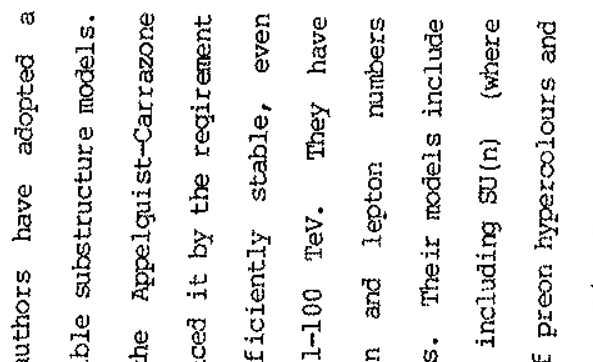

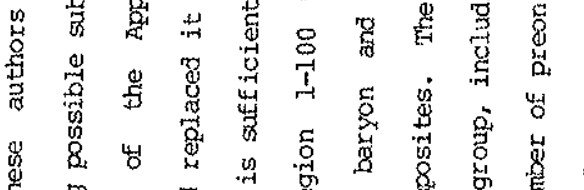

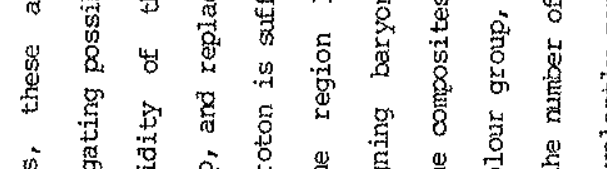

ชั

.

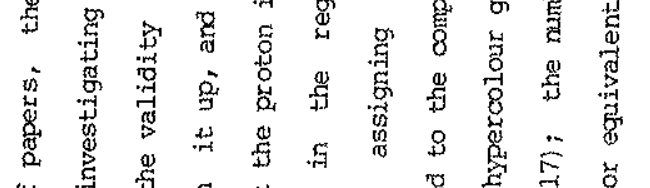

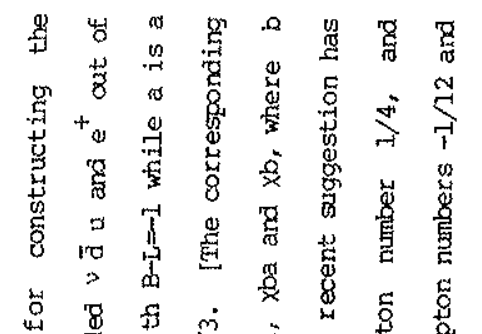

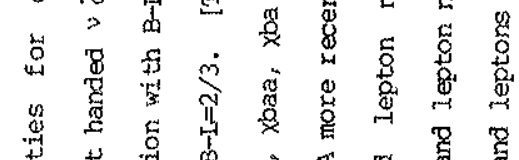

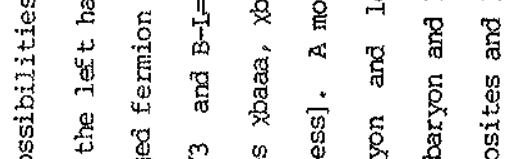

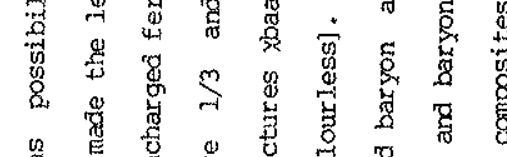

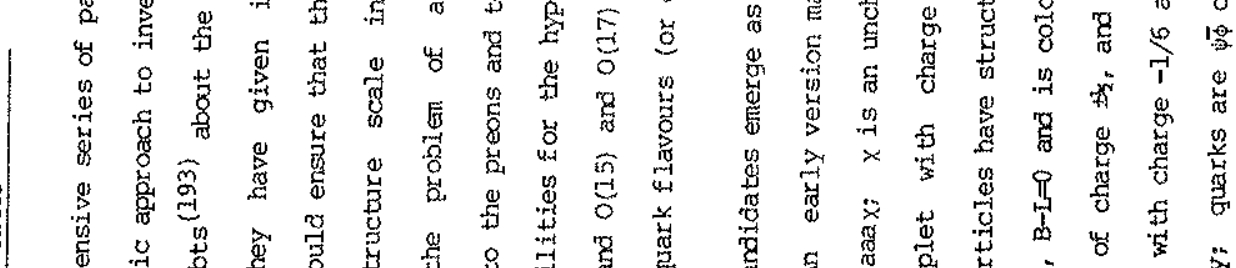

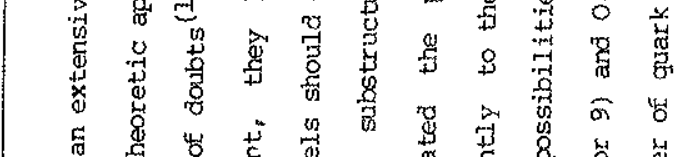

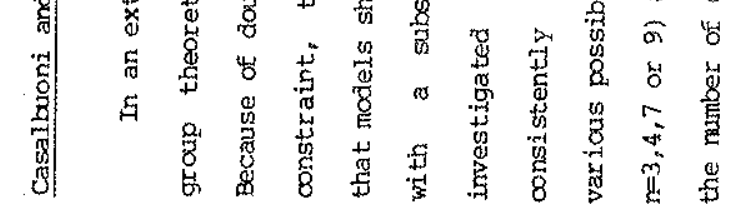

$\frac{1}{3}$

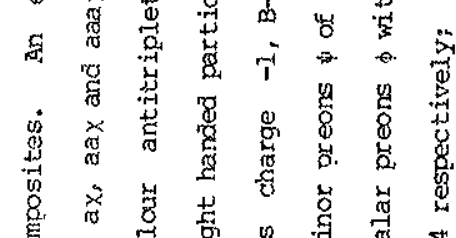

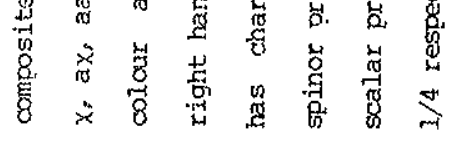

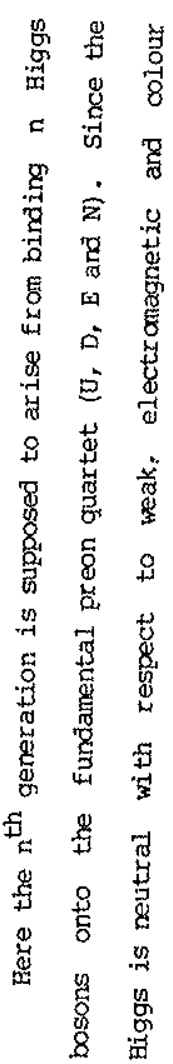

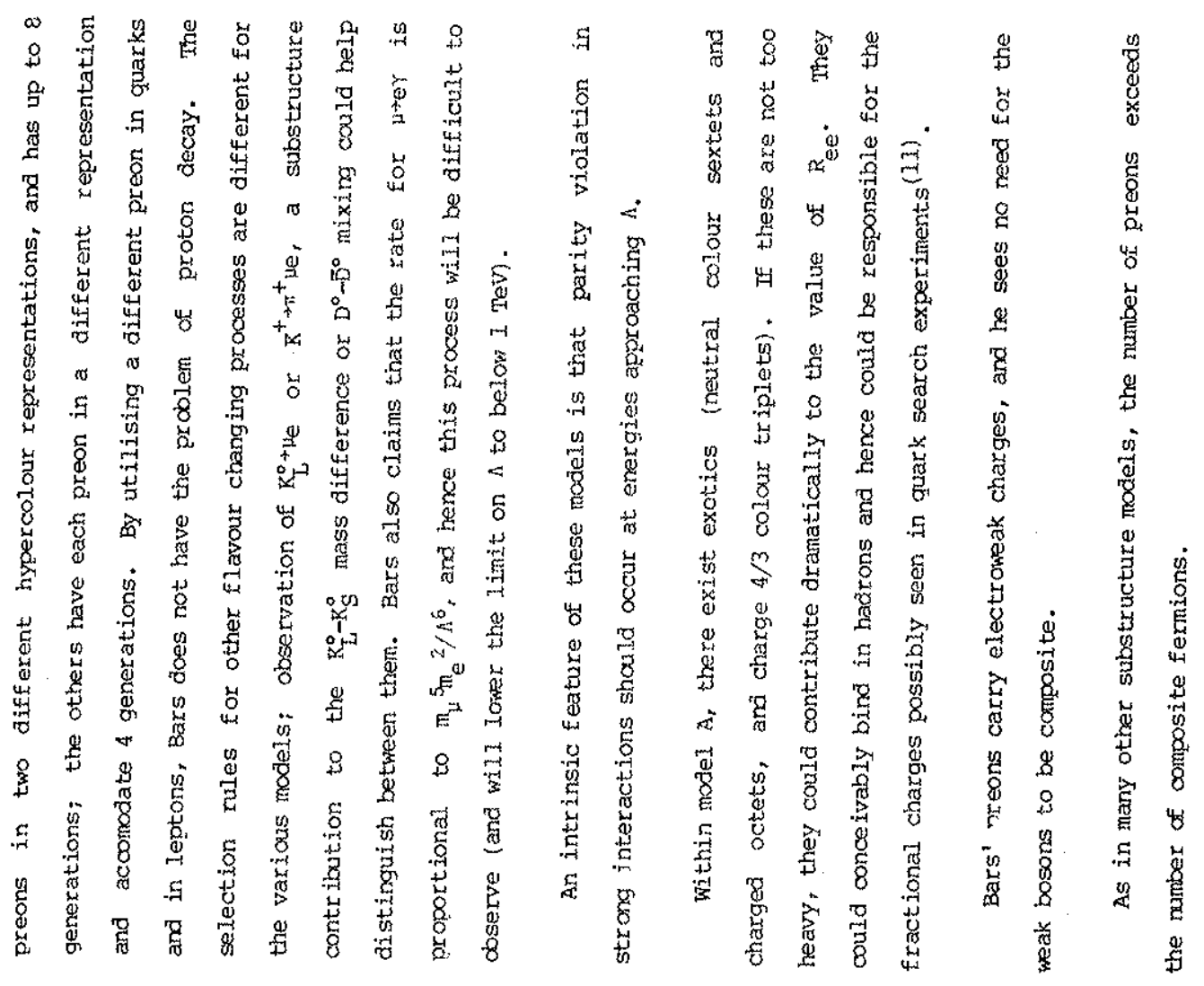



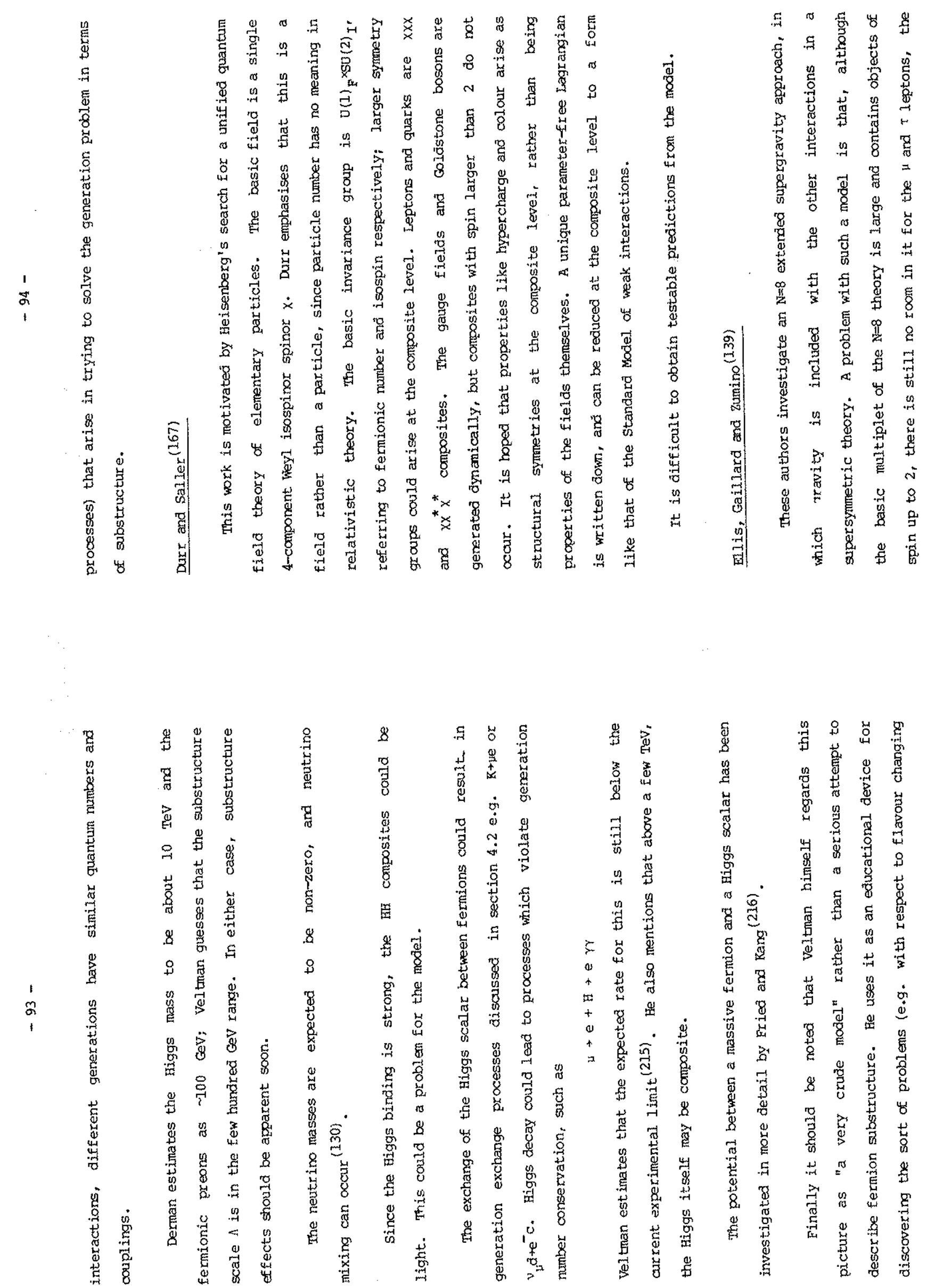


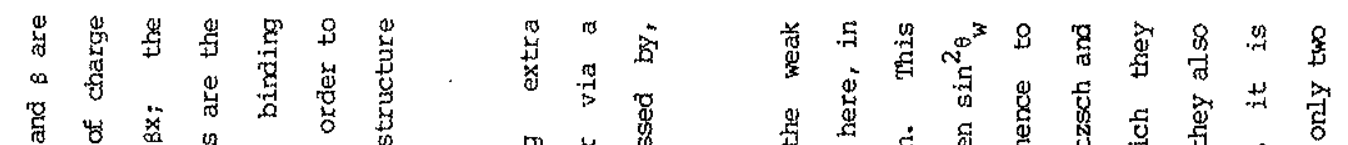

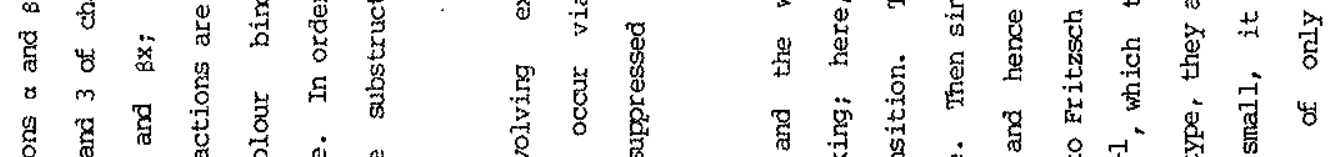

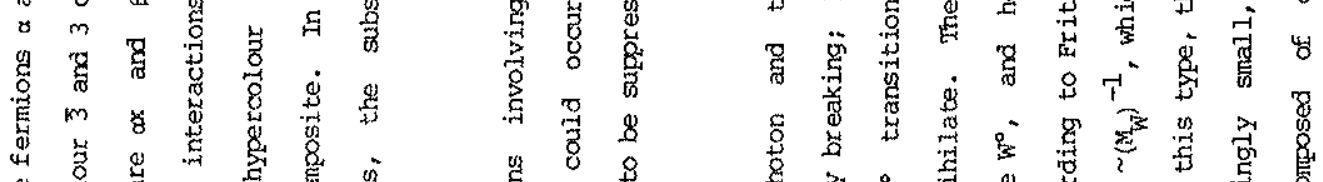

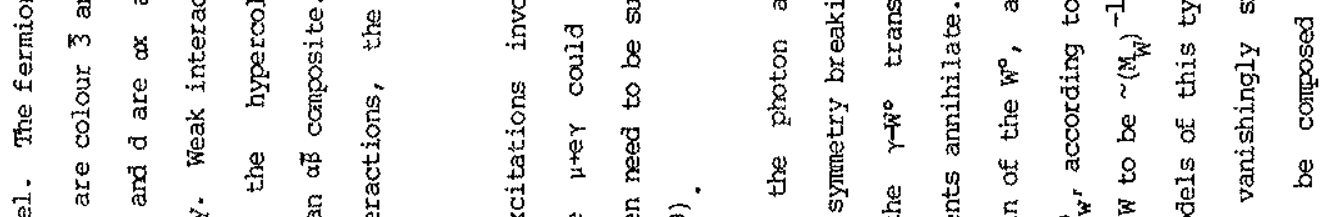

$$
\begin{aligned}
& \text { 1) }
\end{aligned}
$$

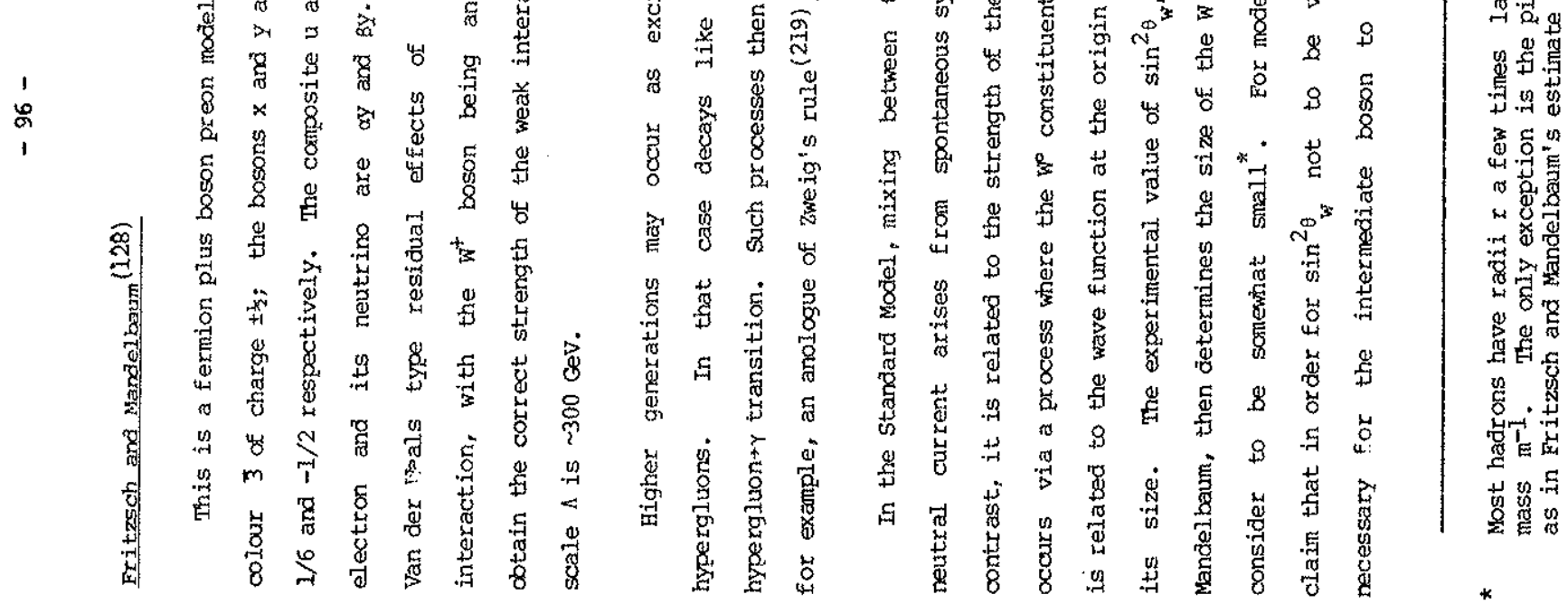

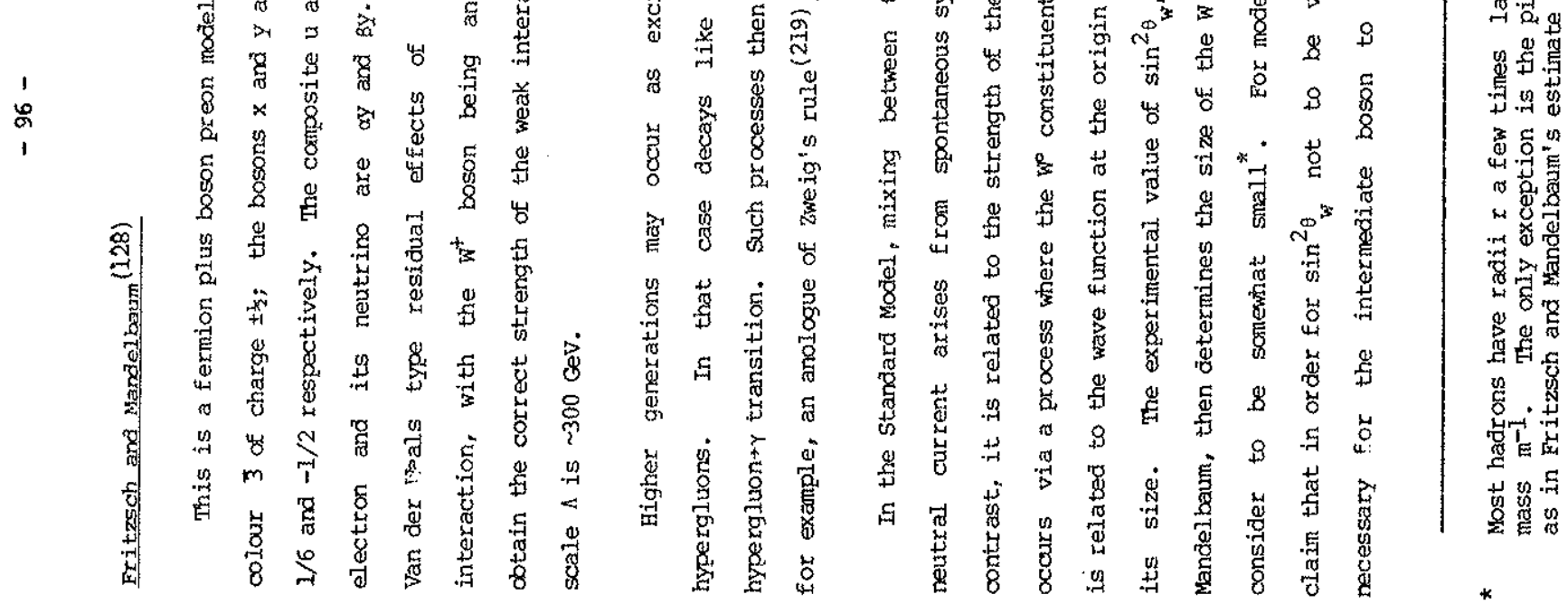

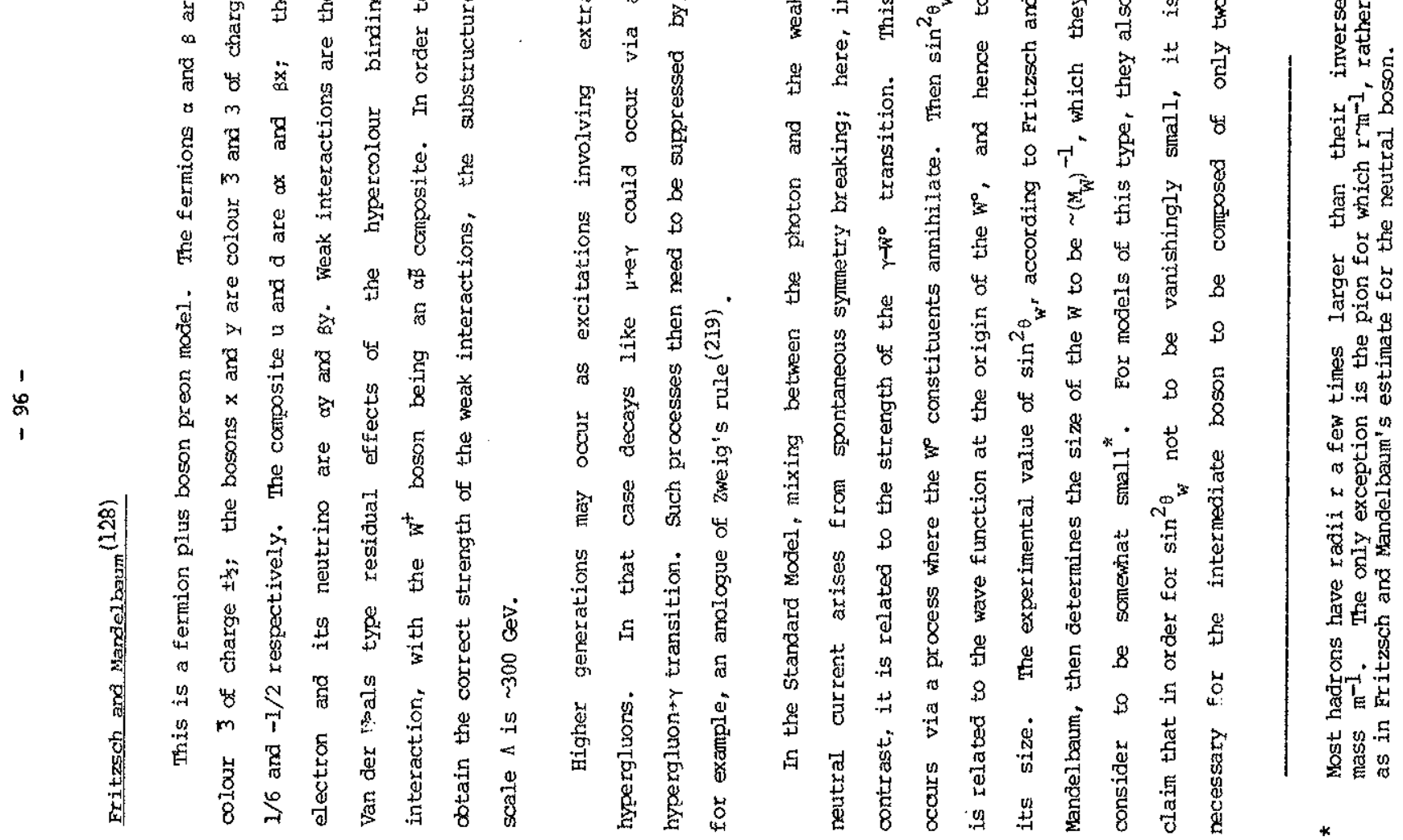

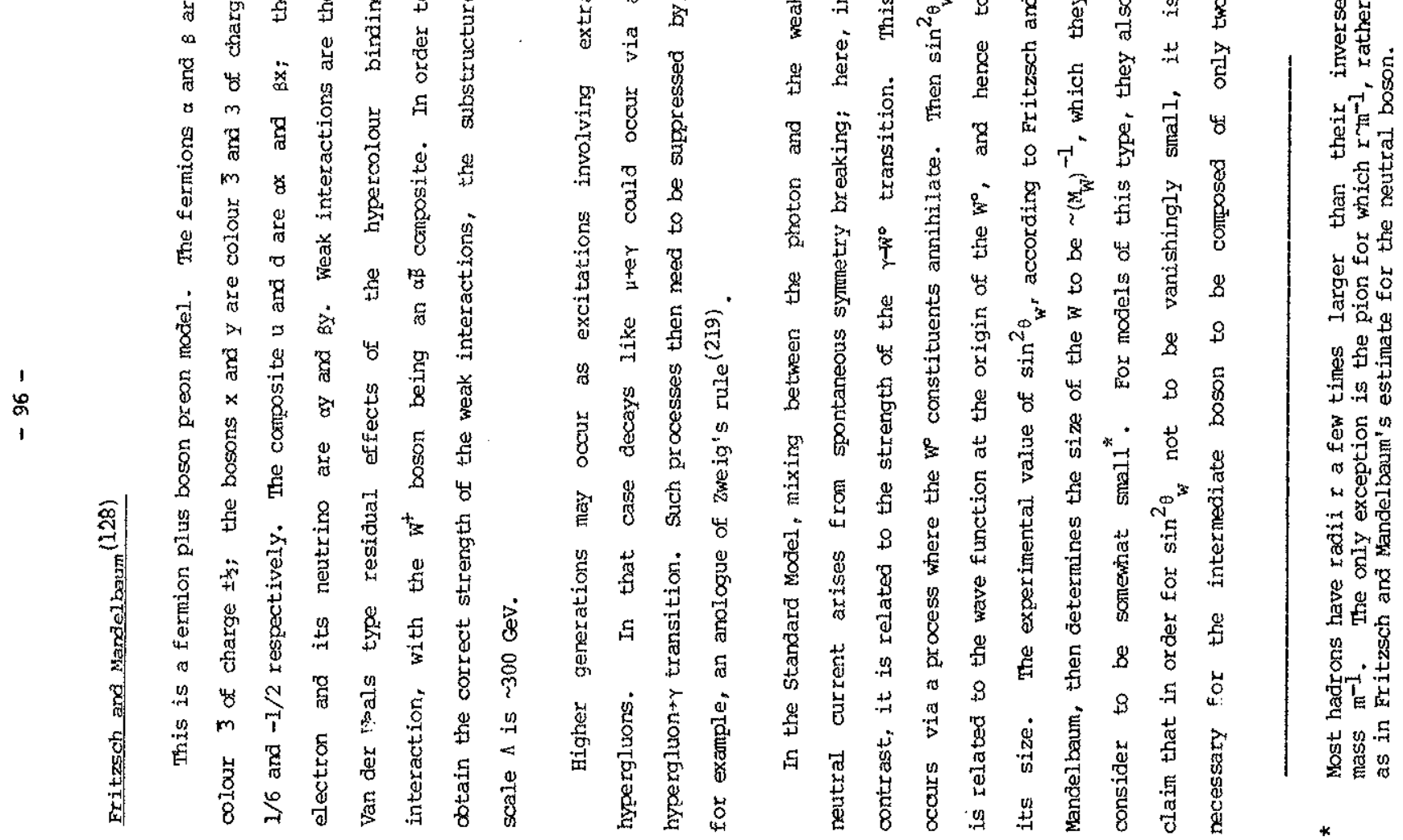

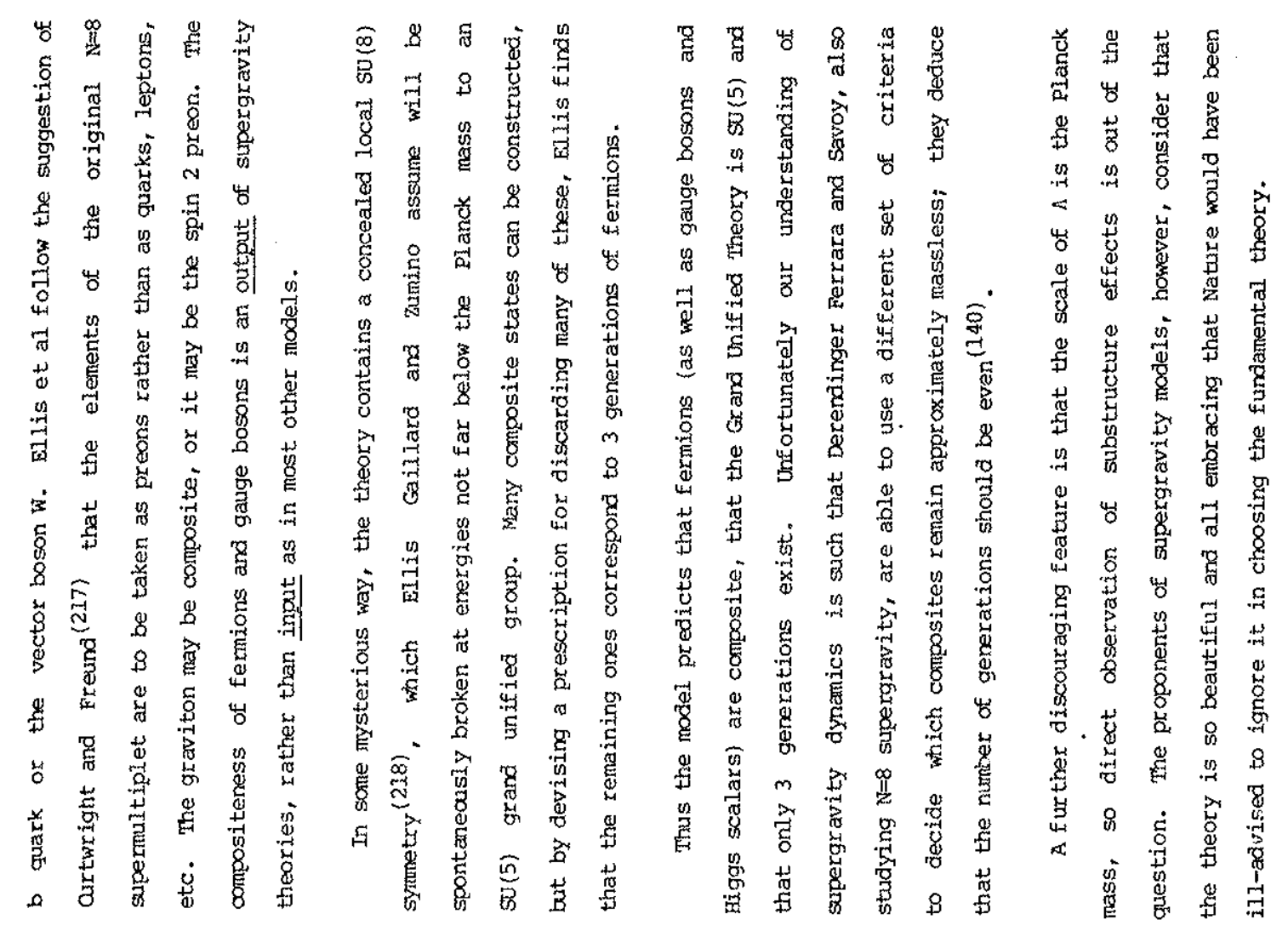




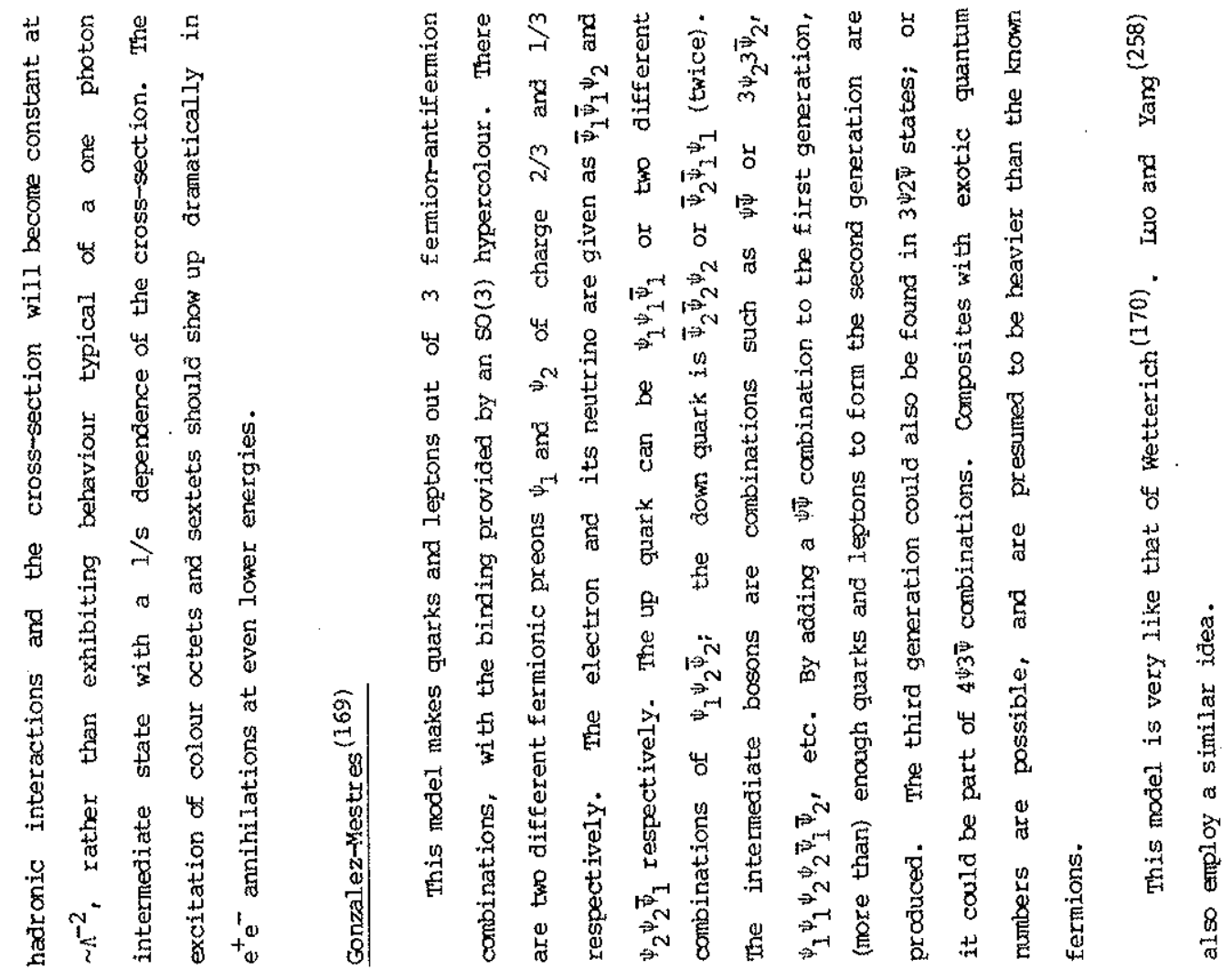

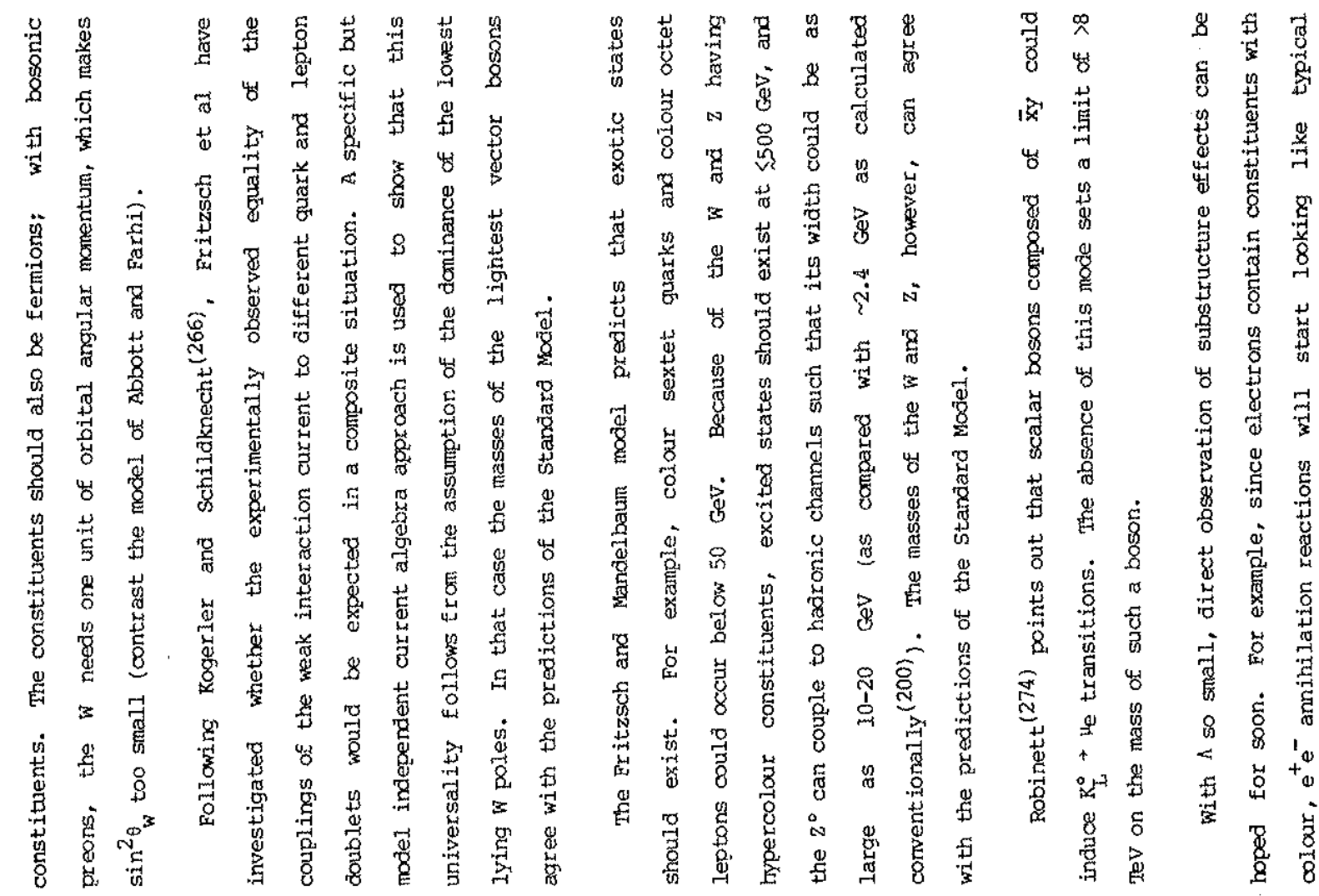




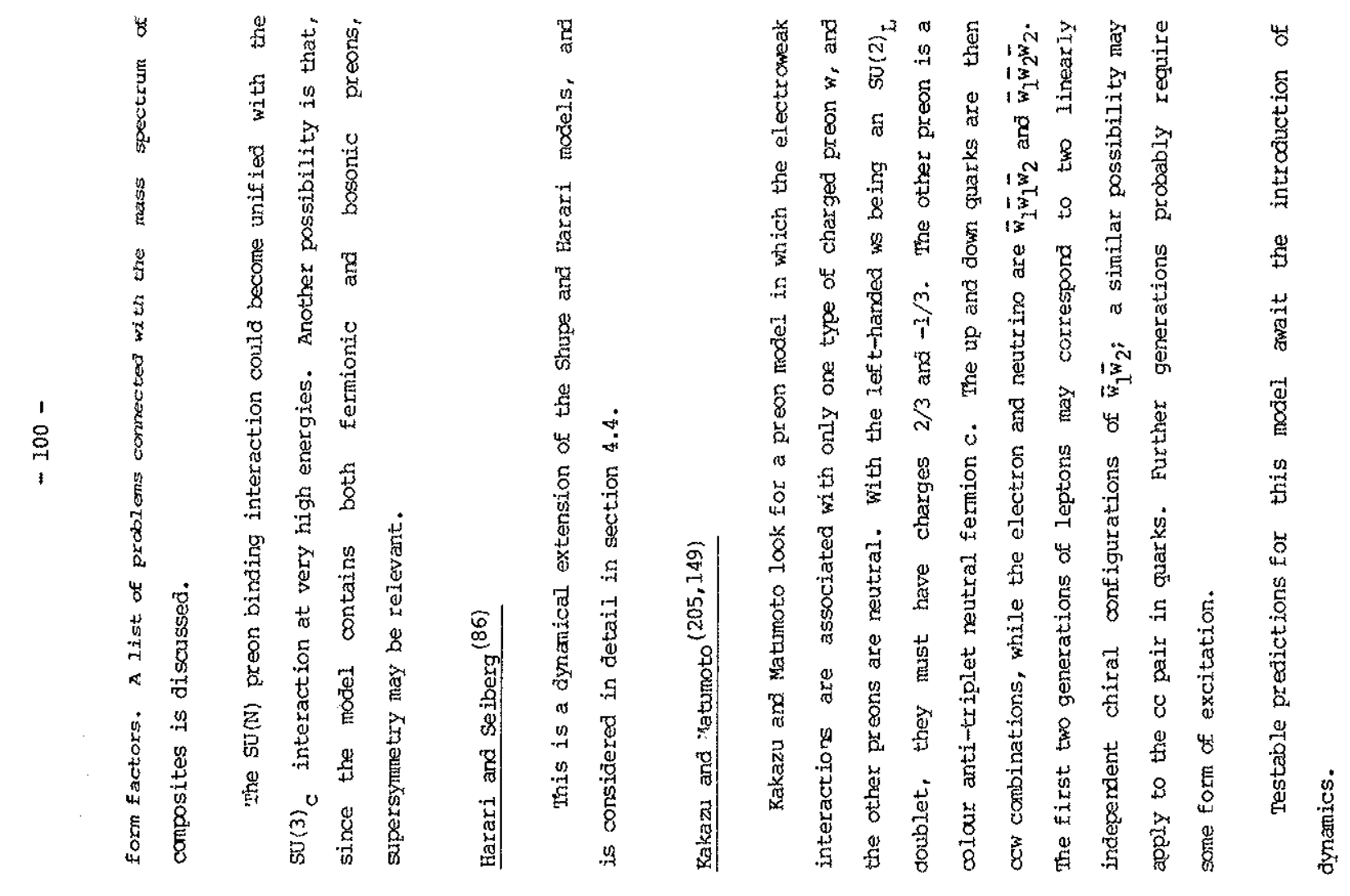

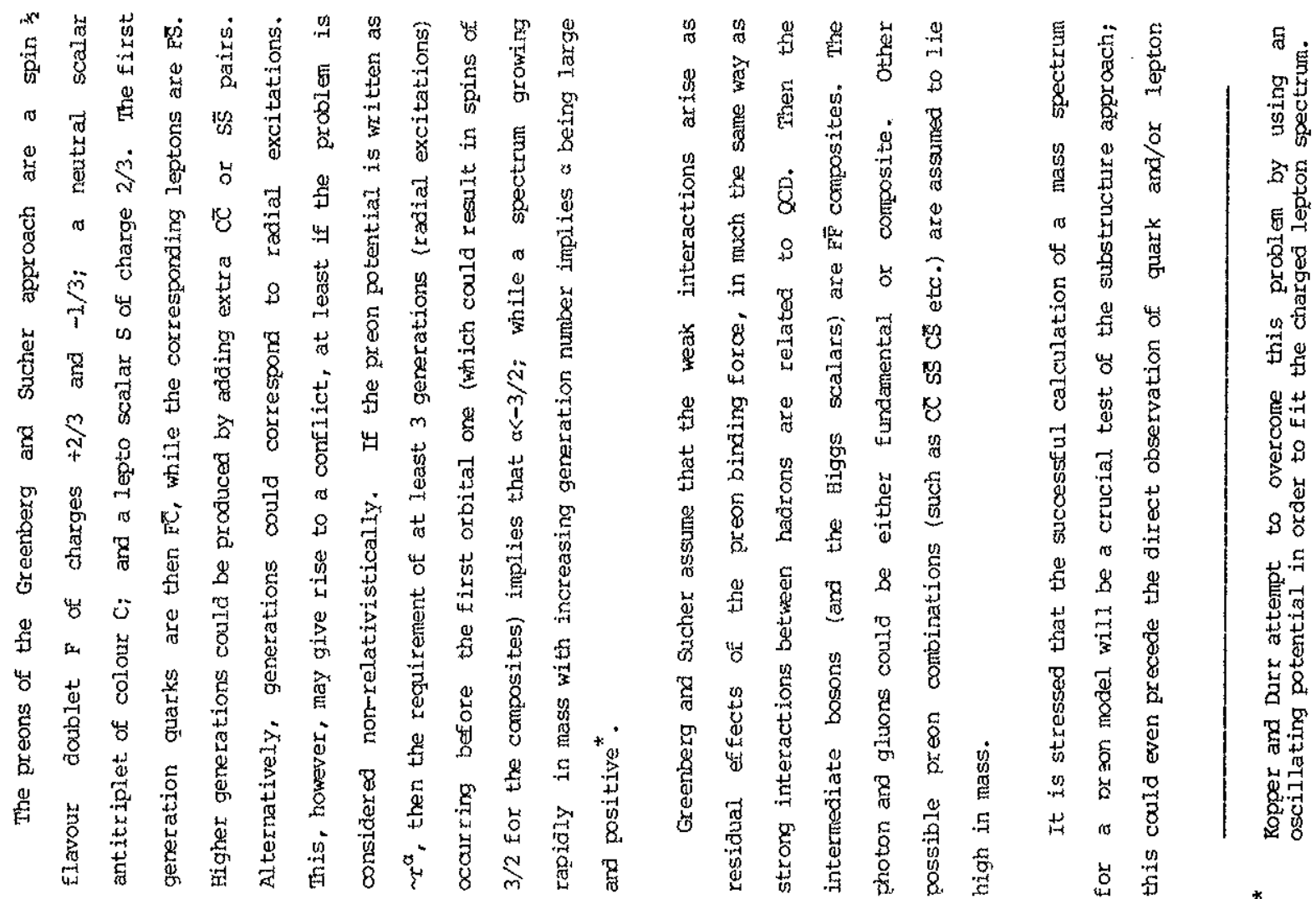



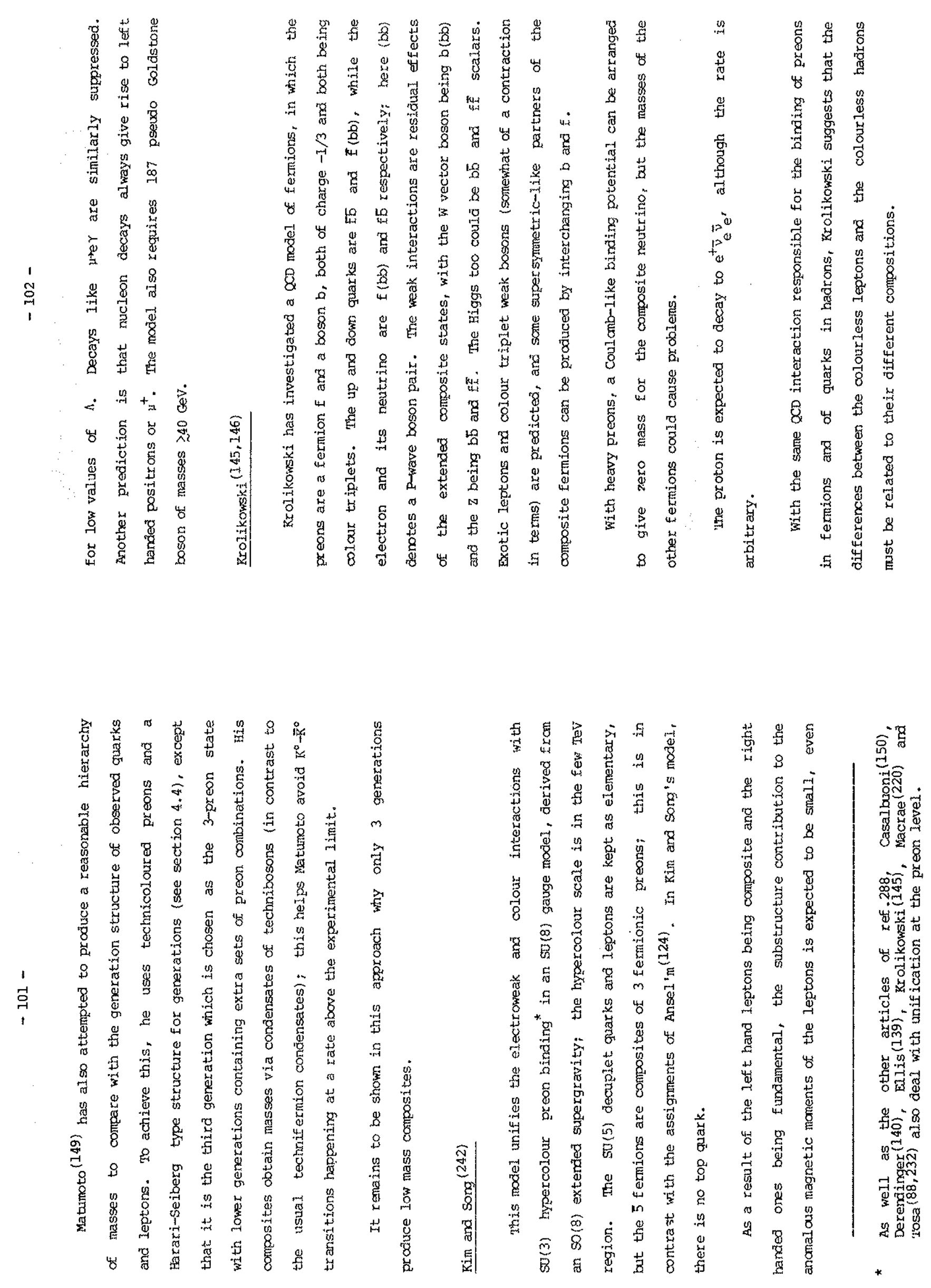

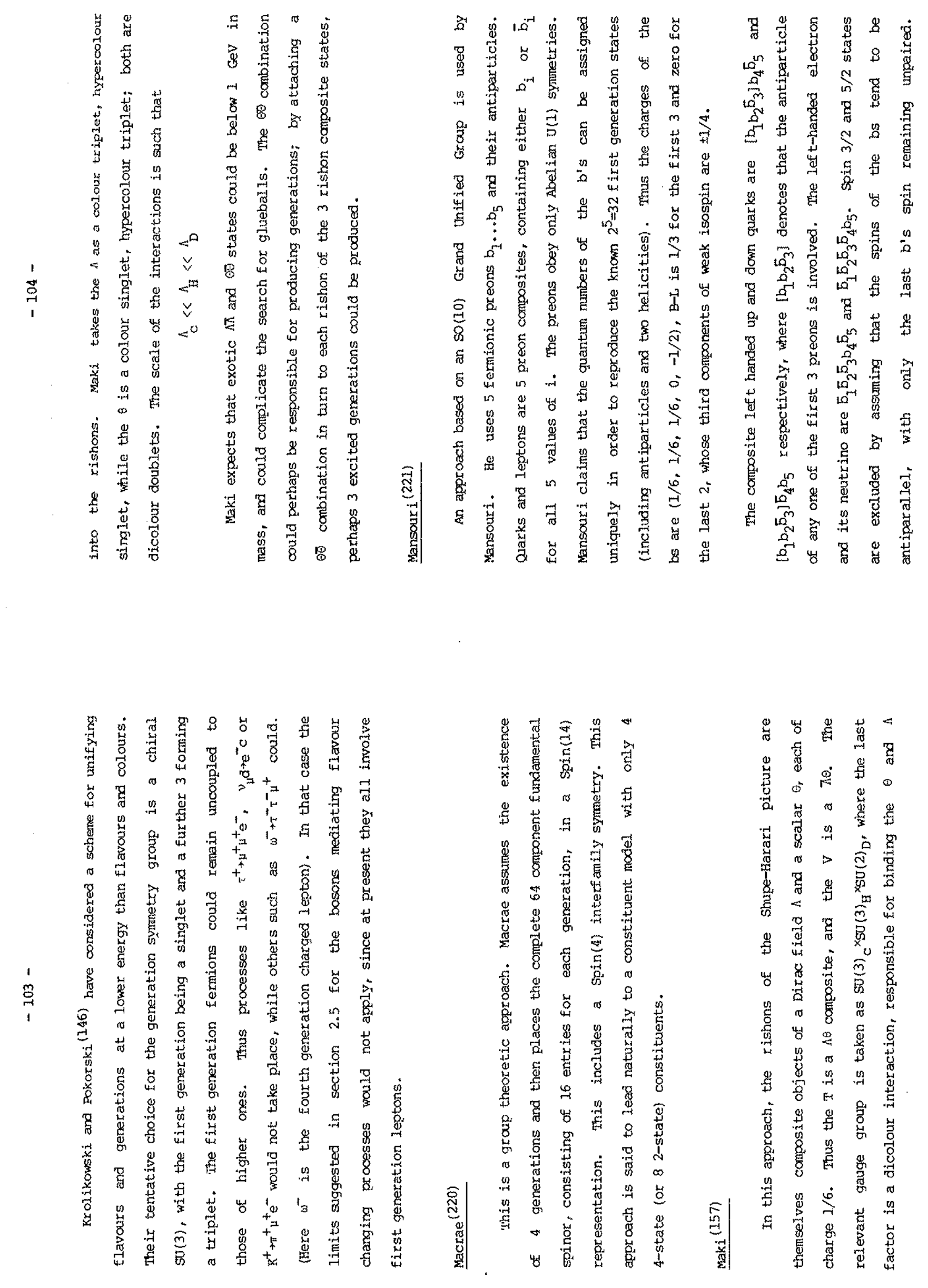

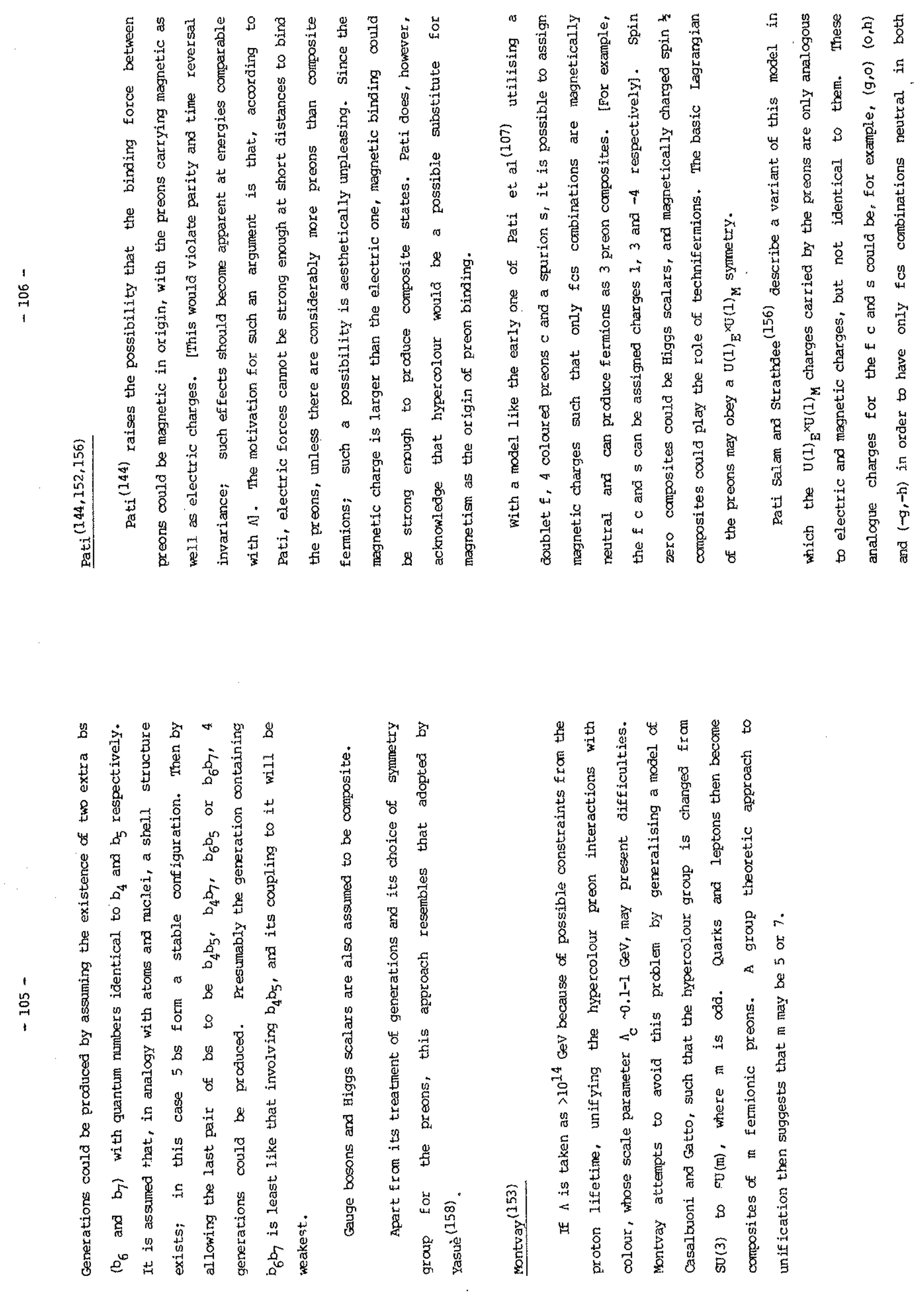


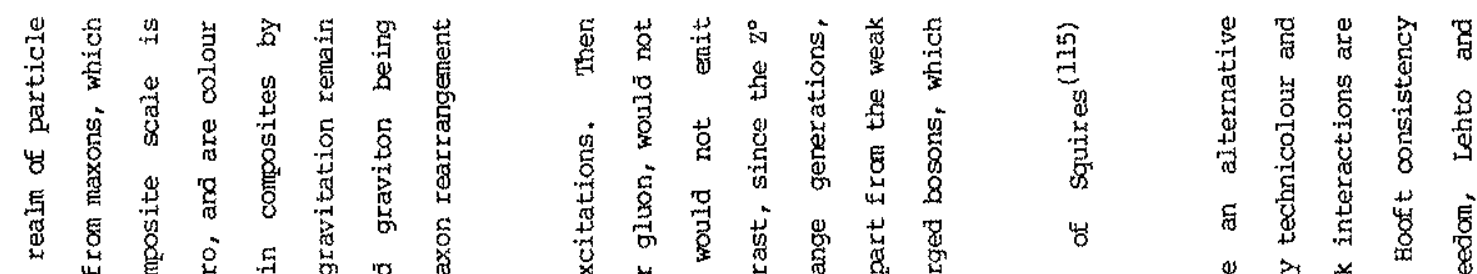

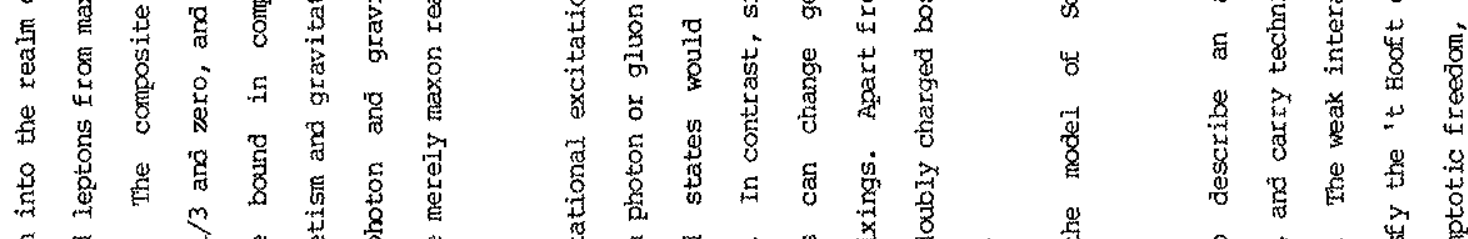

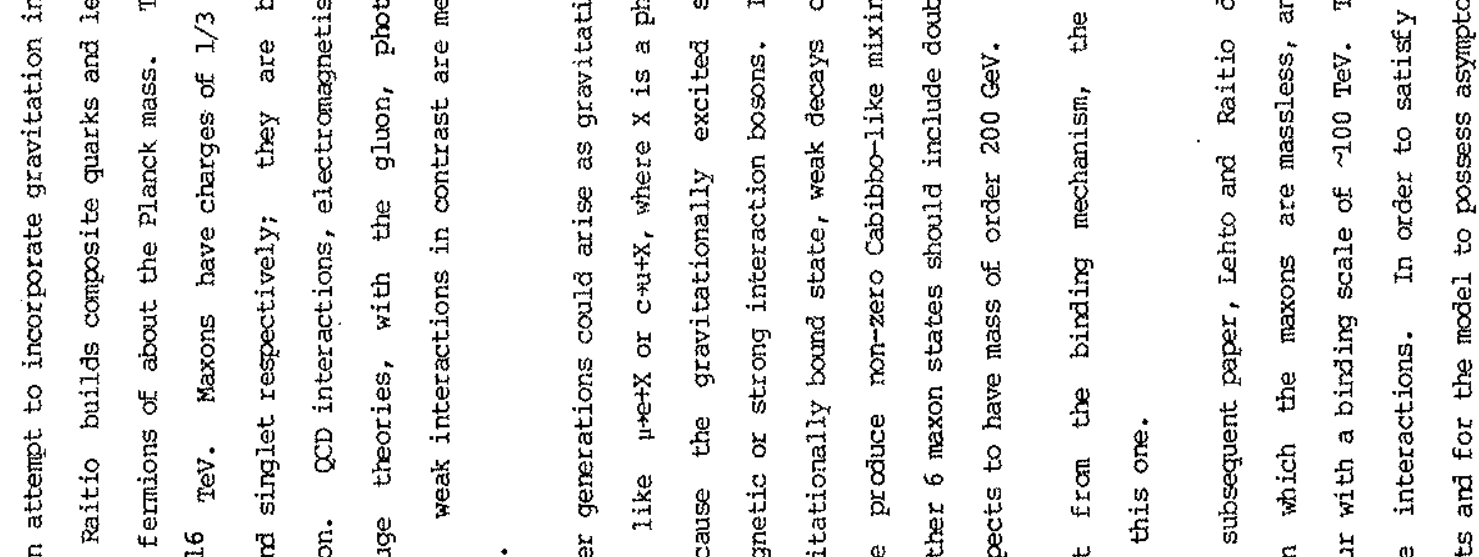

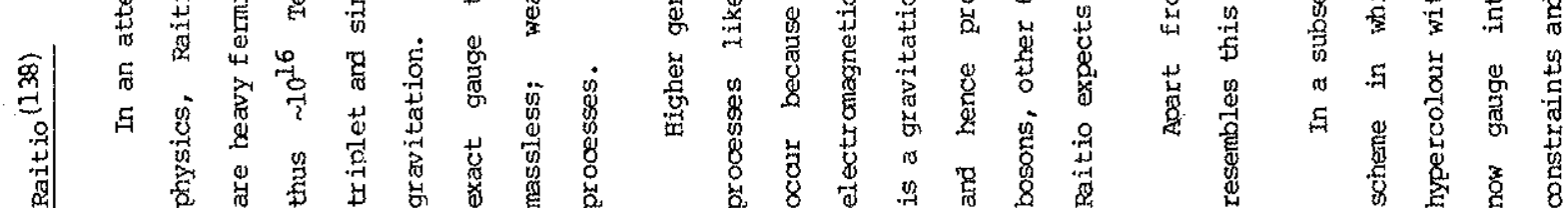

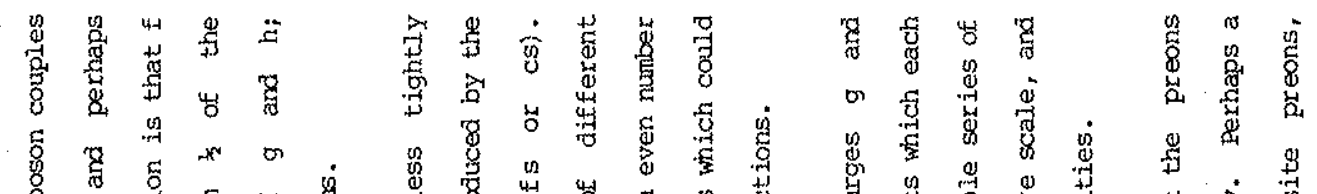

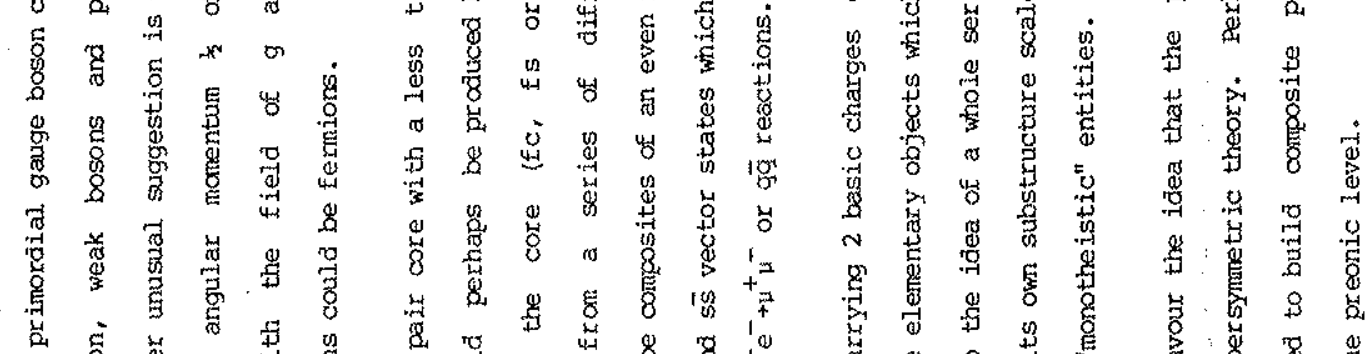

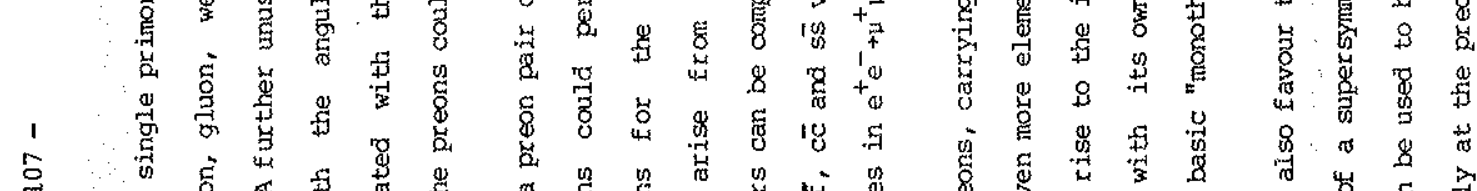

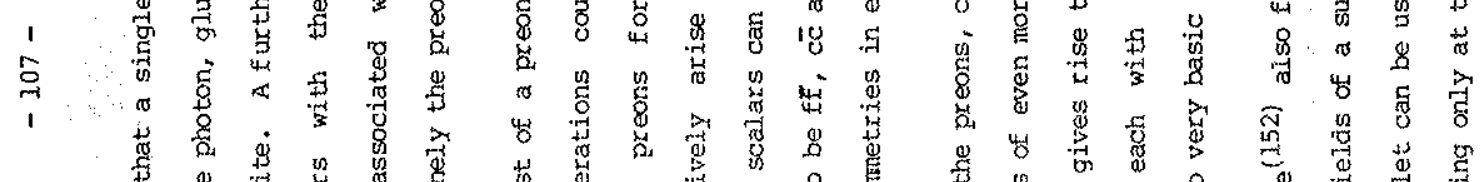

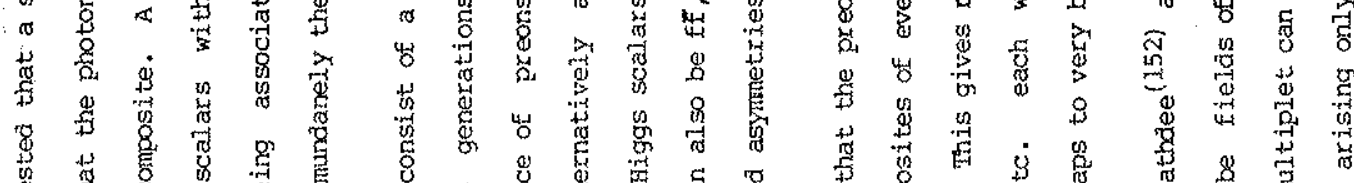

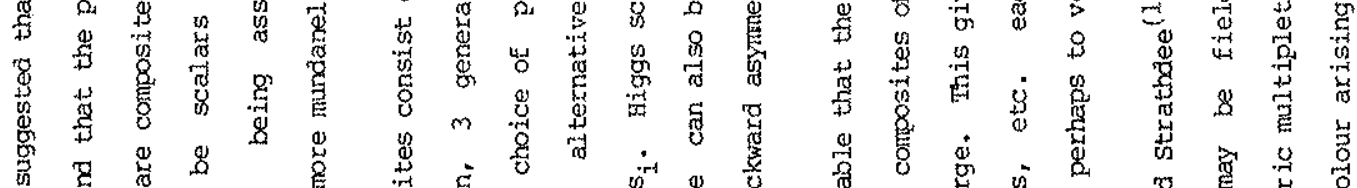

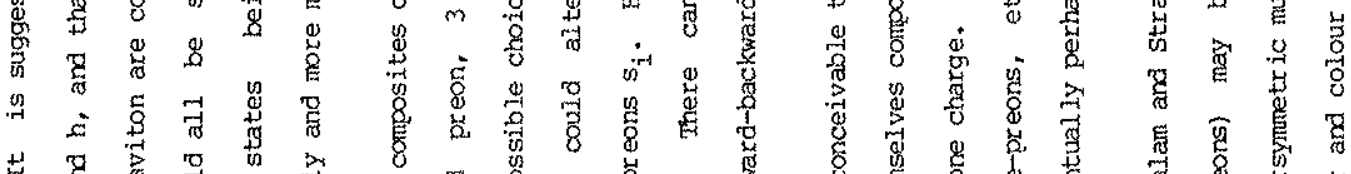

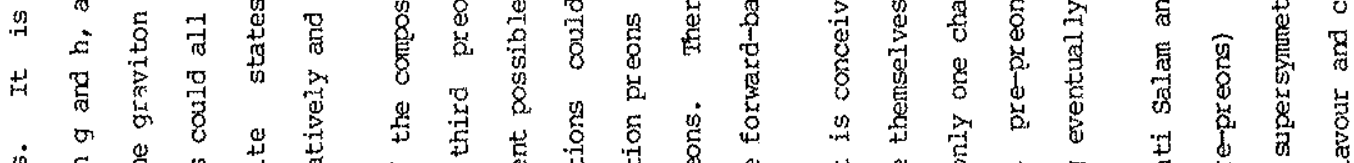

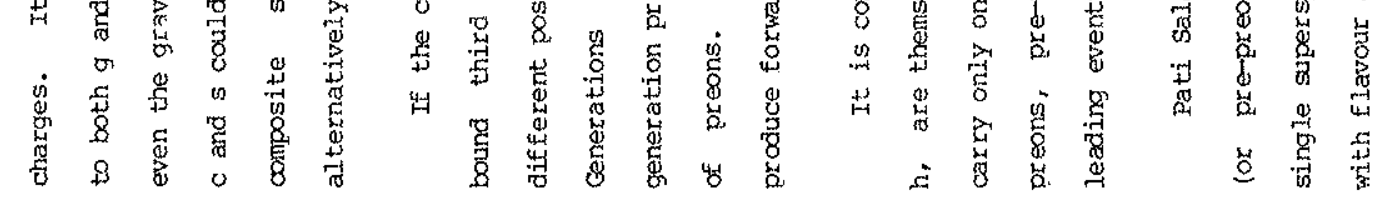




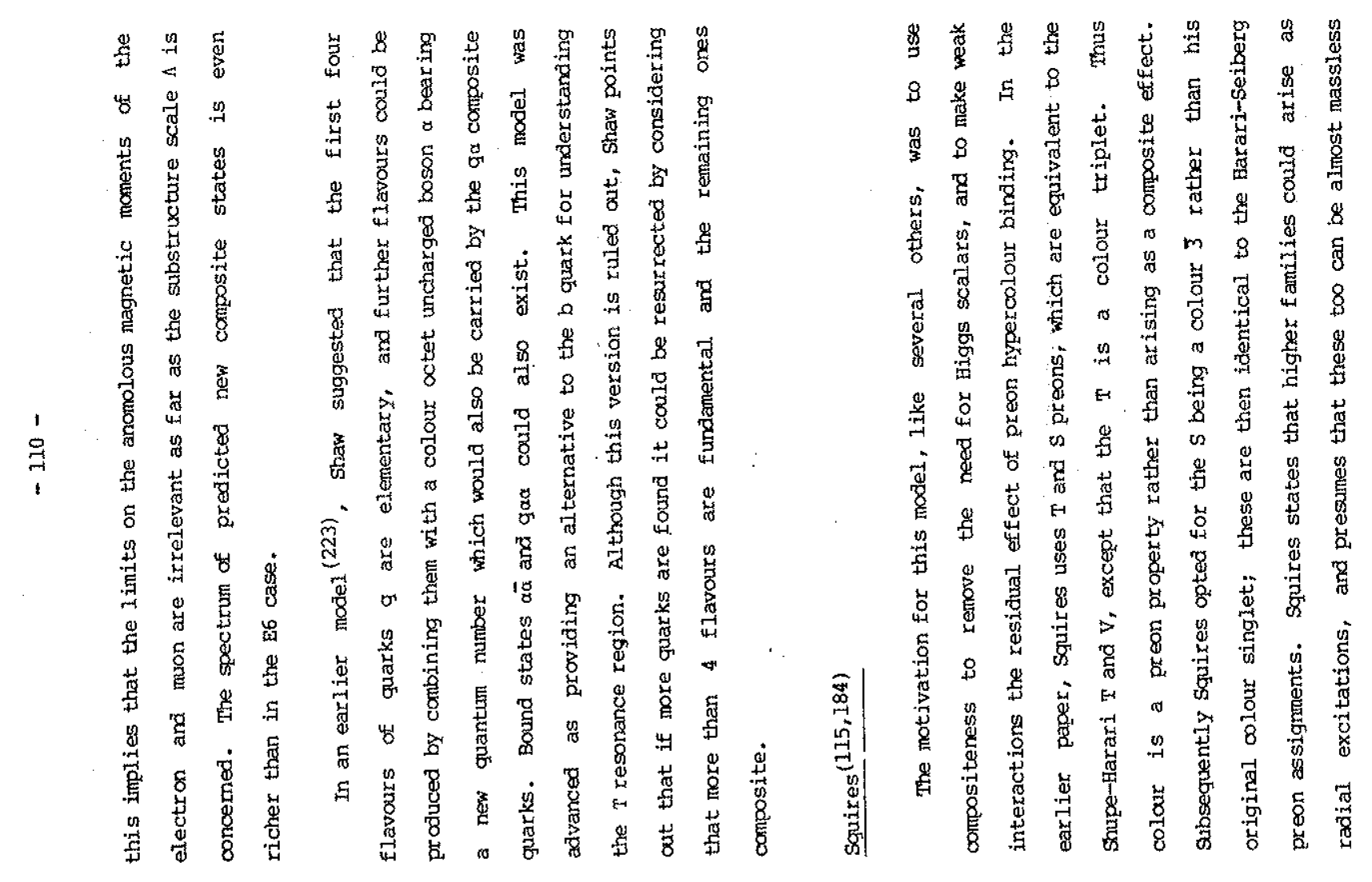

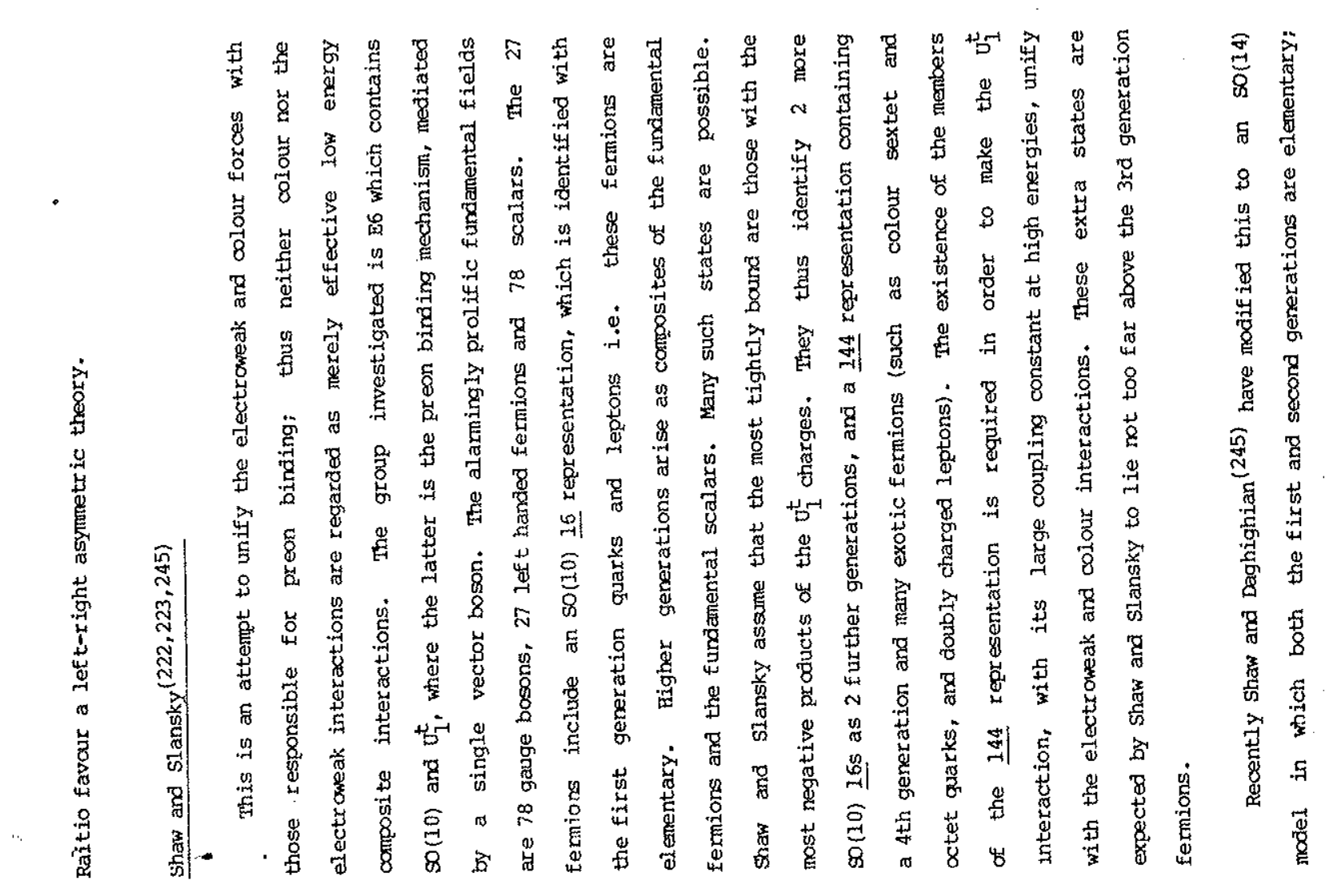




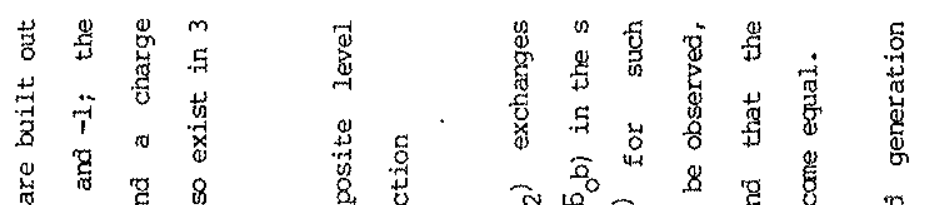

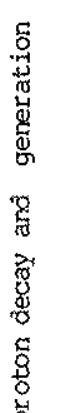

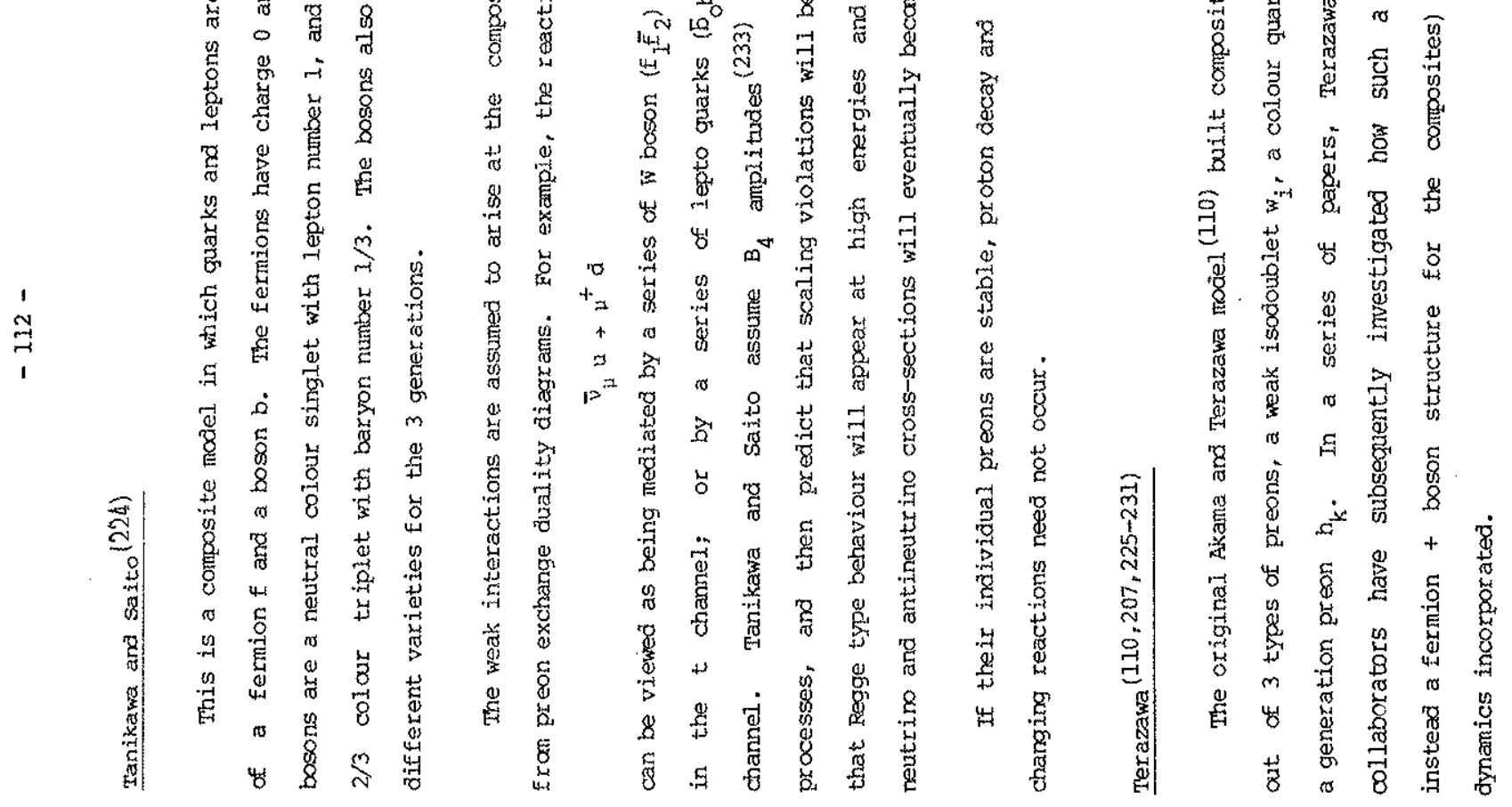

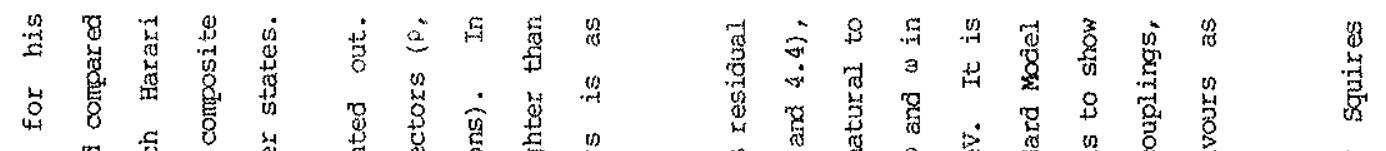

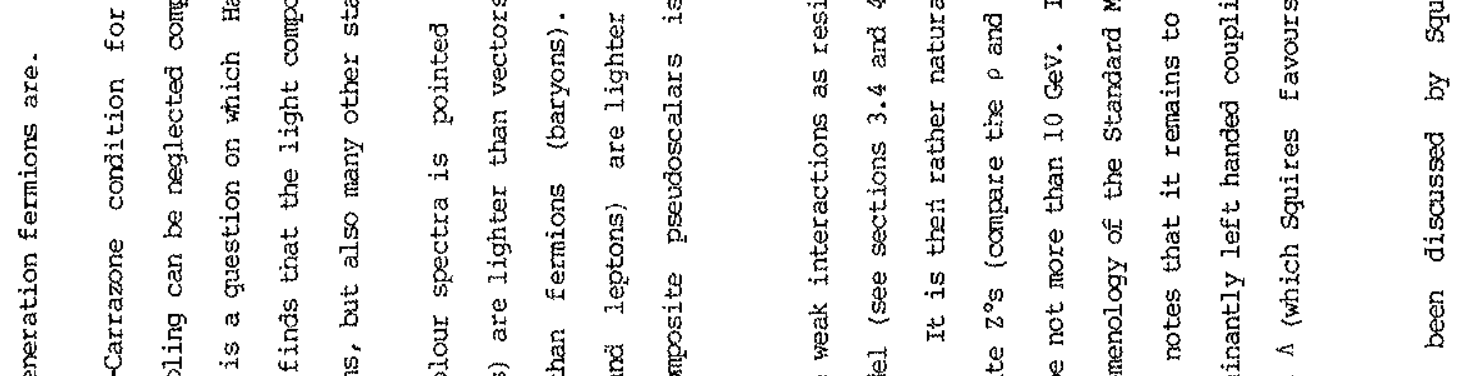

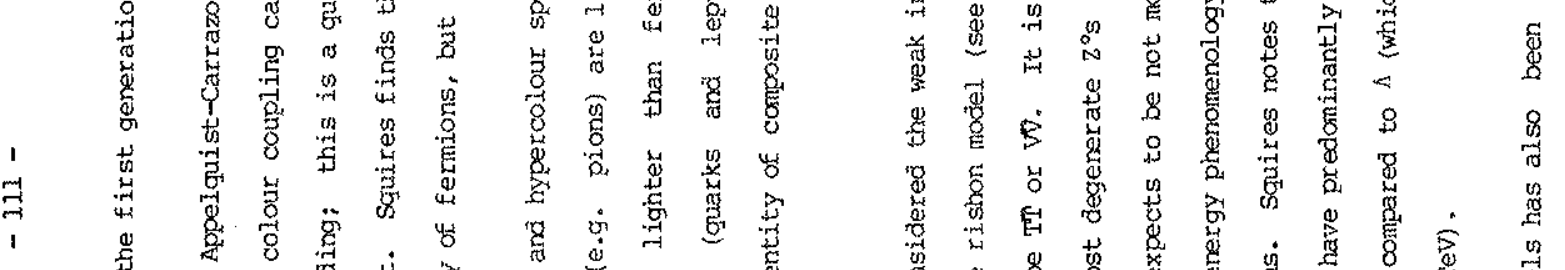

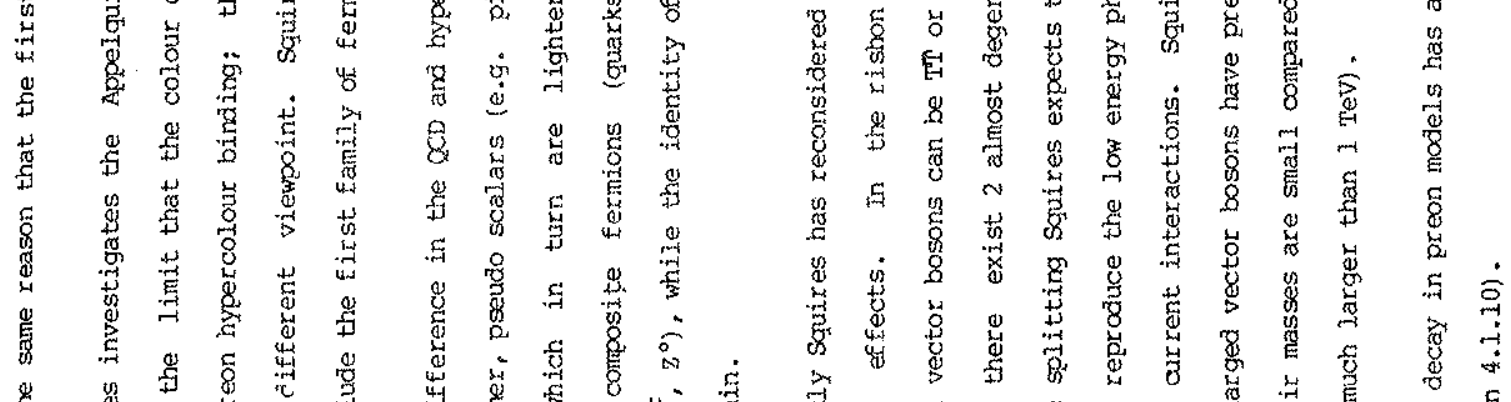

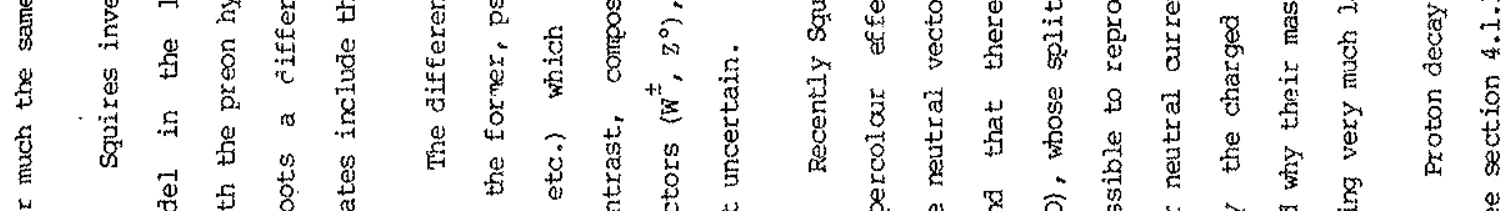

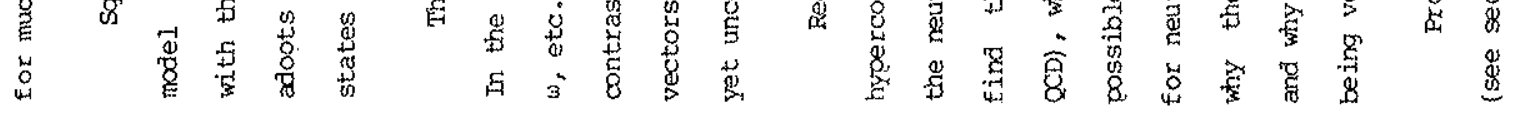



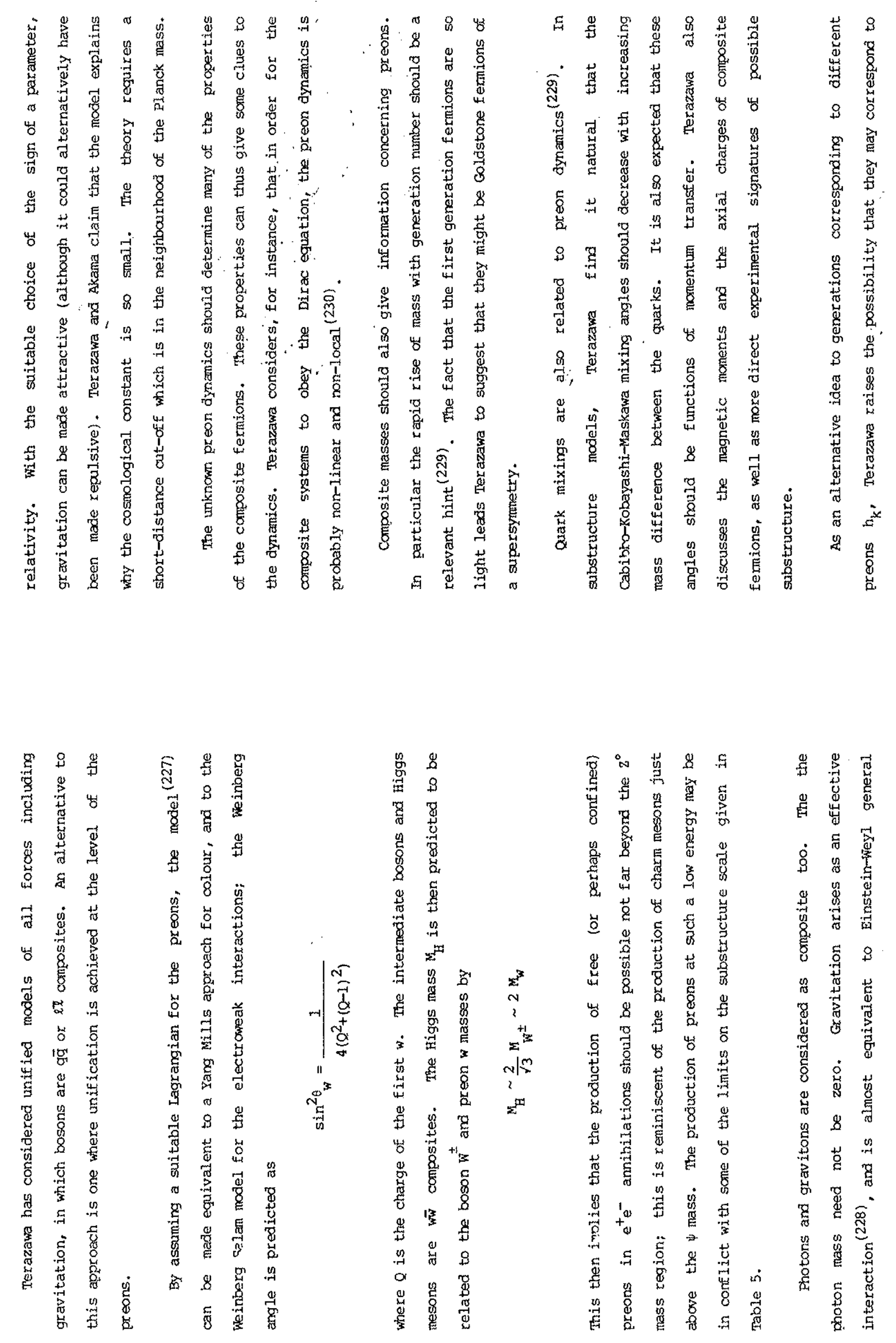


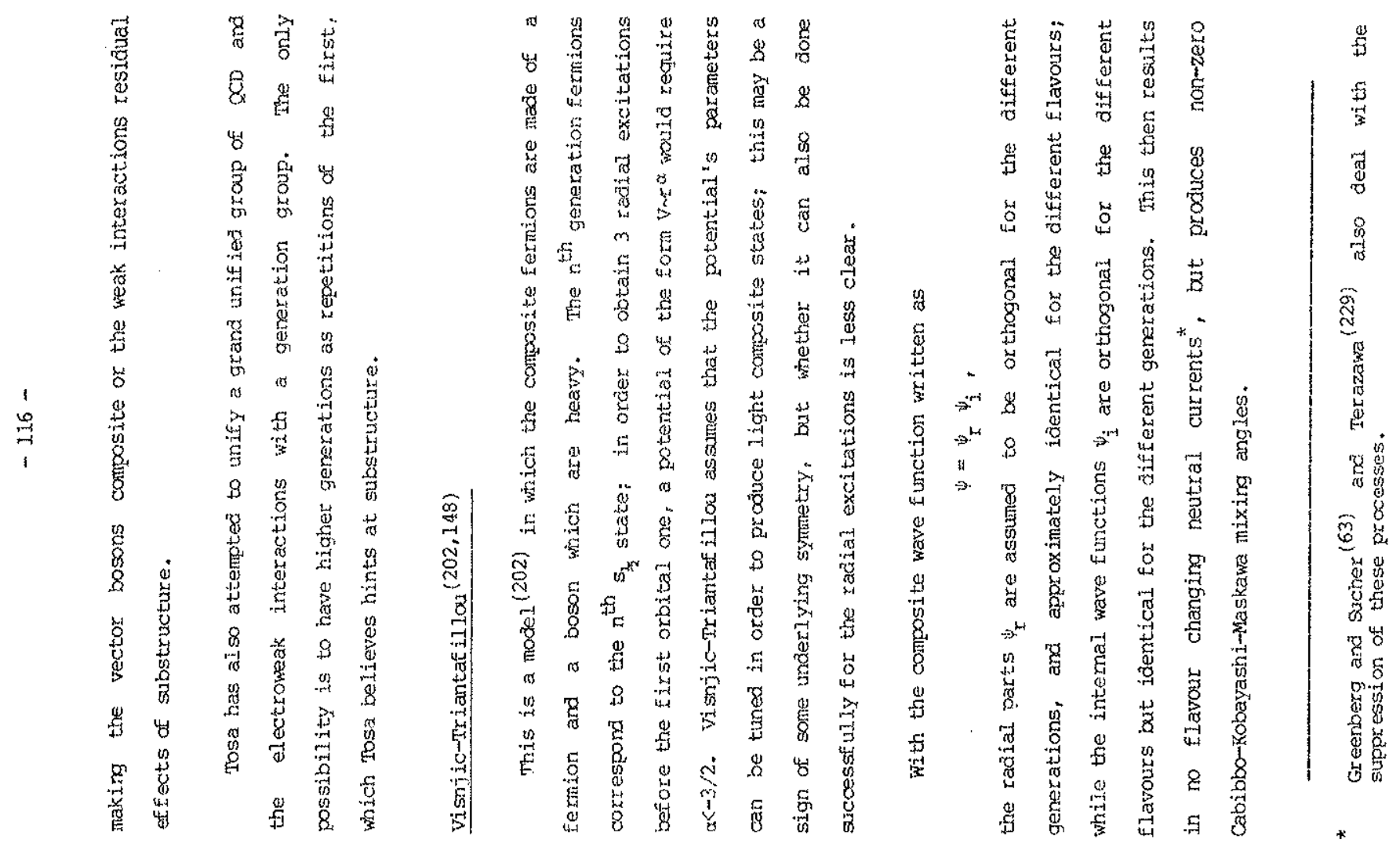

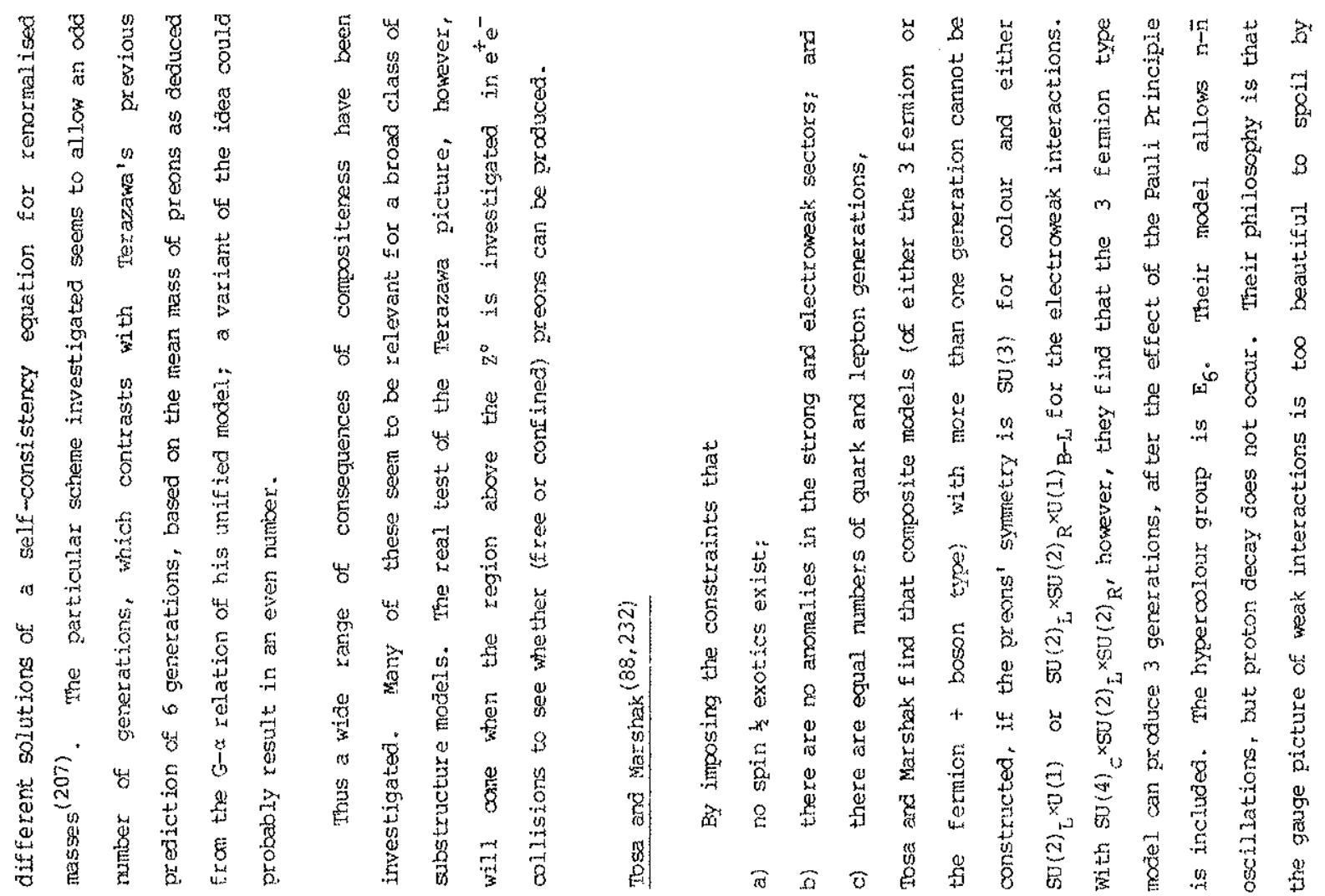



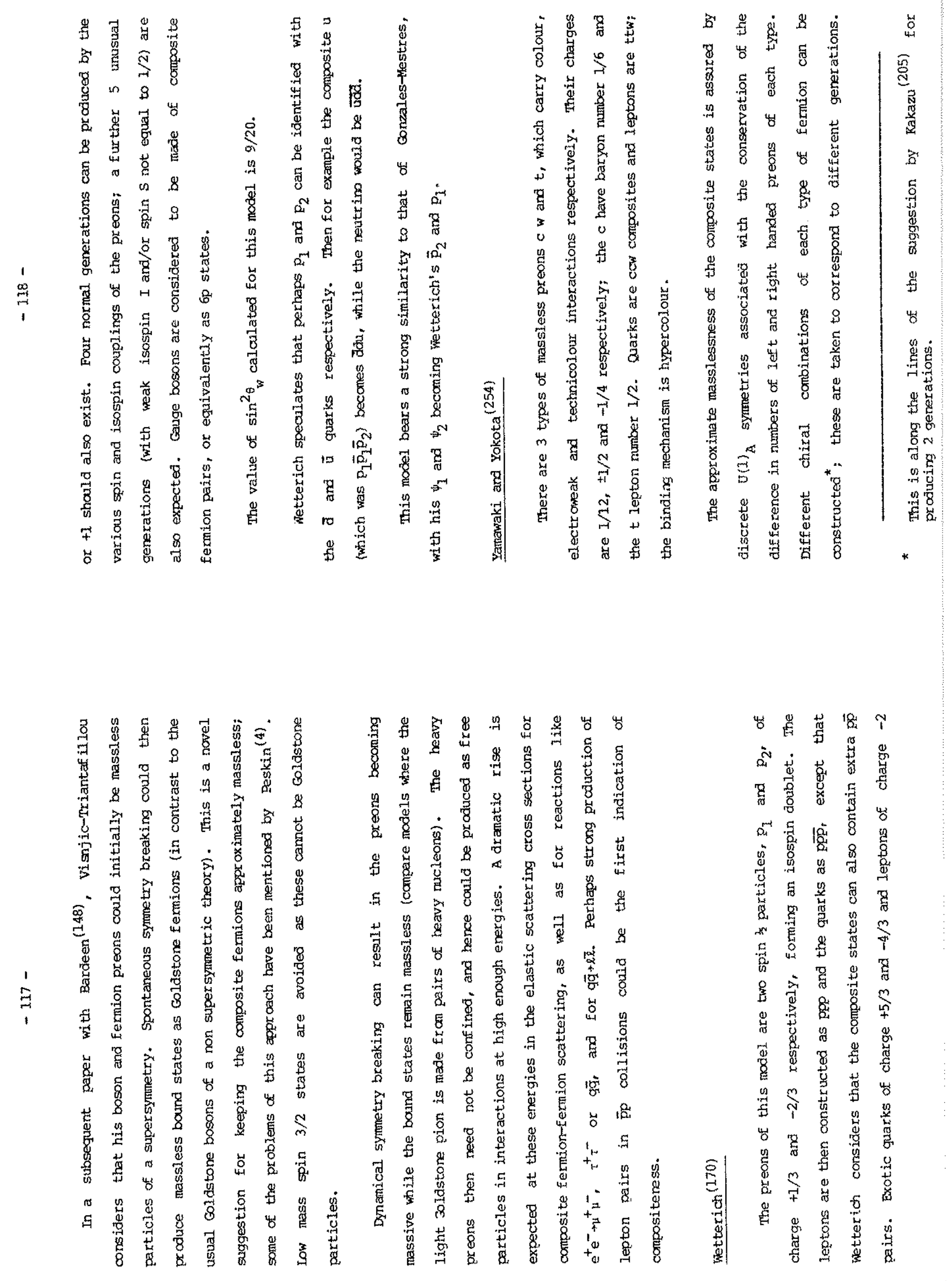

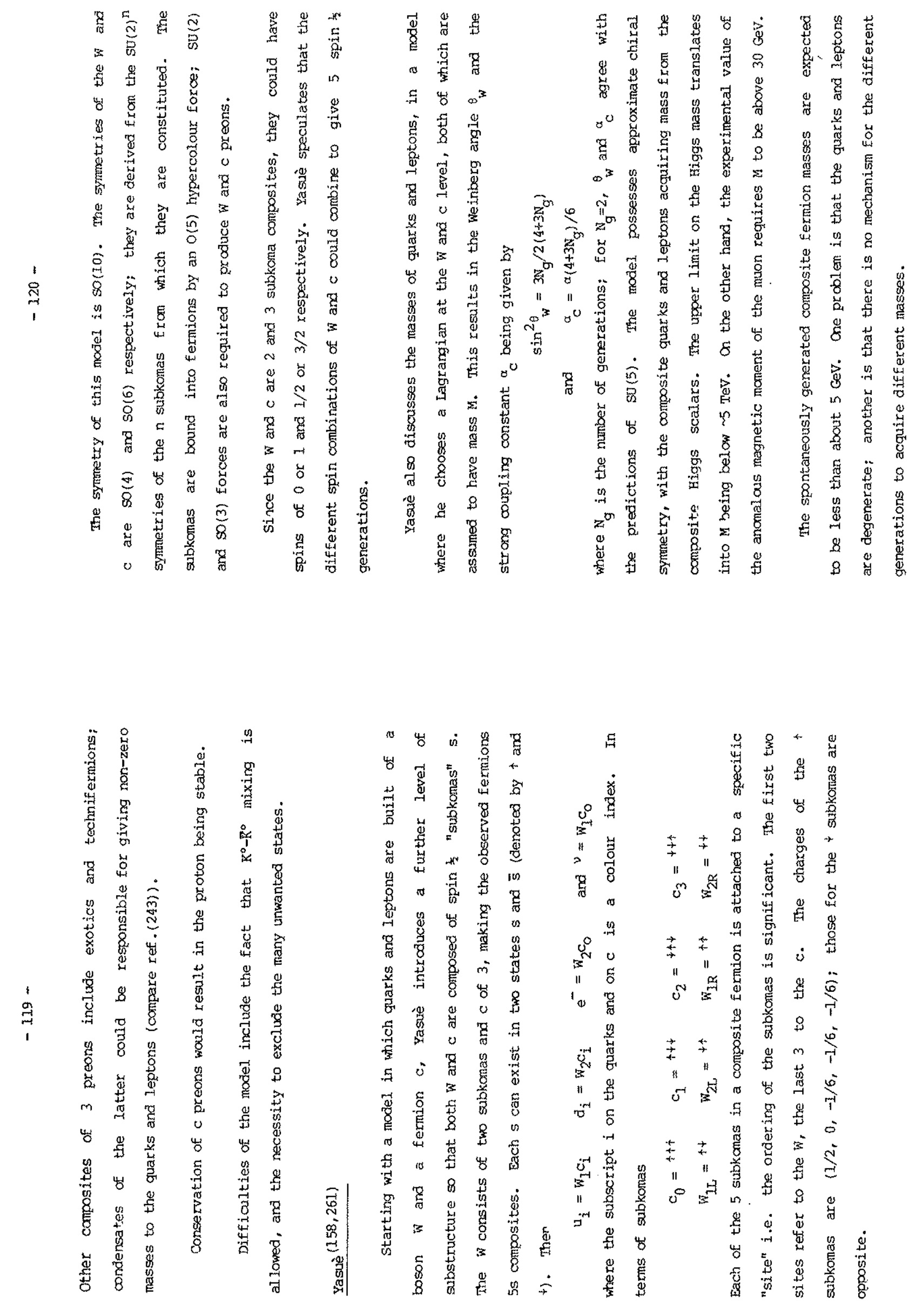

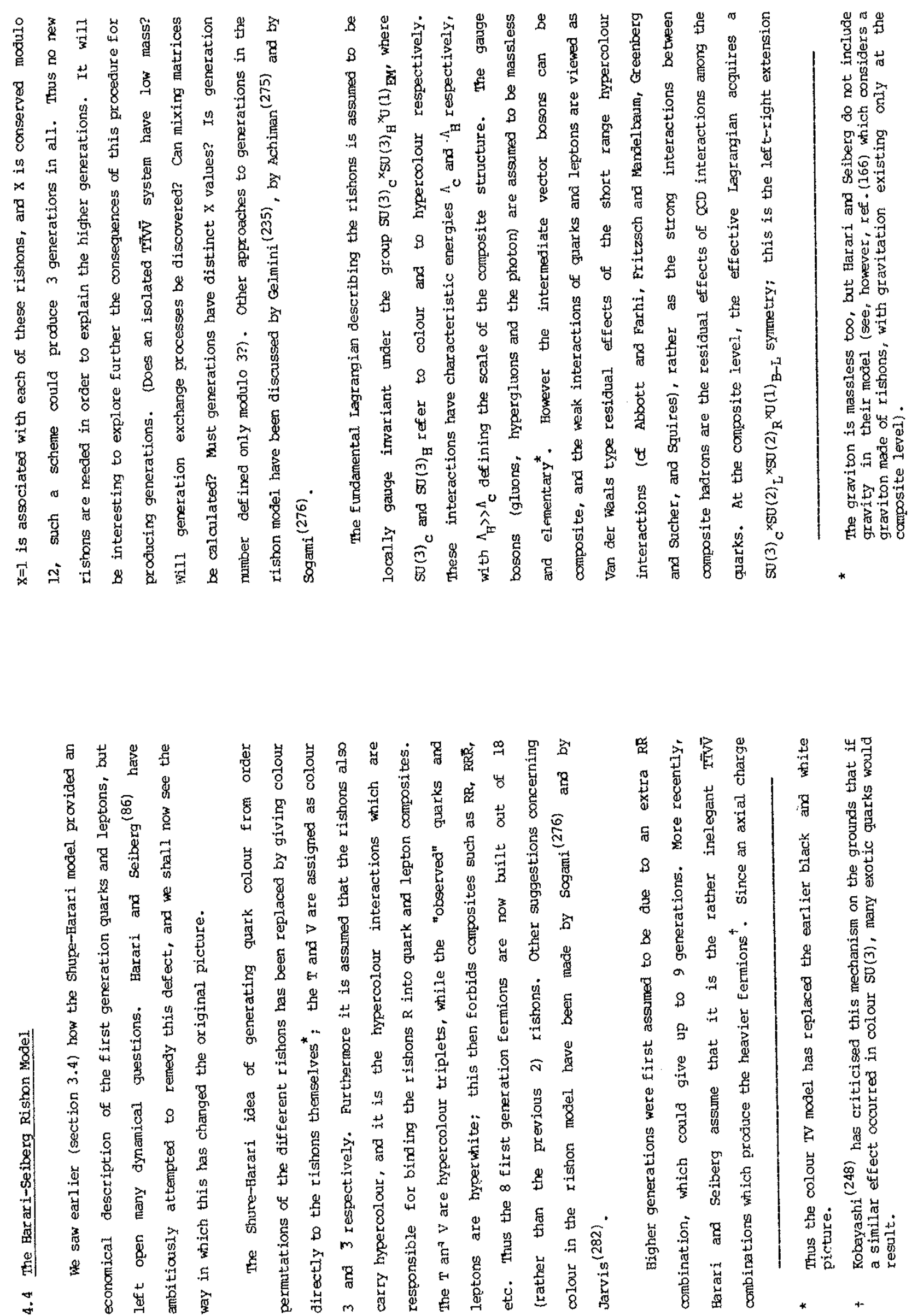

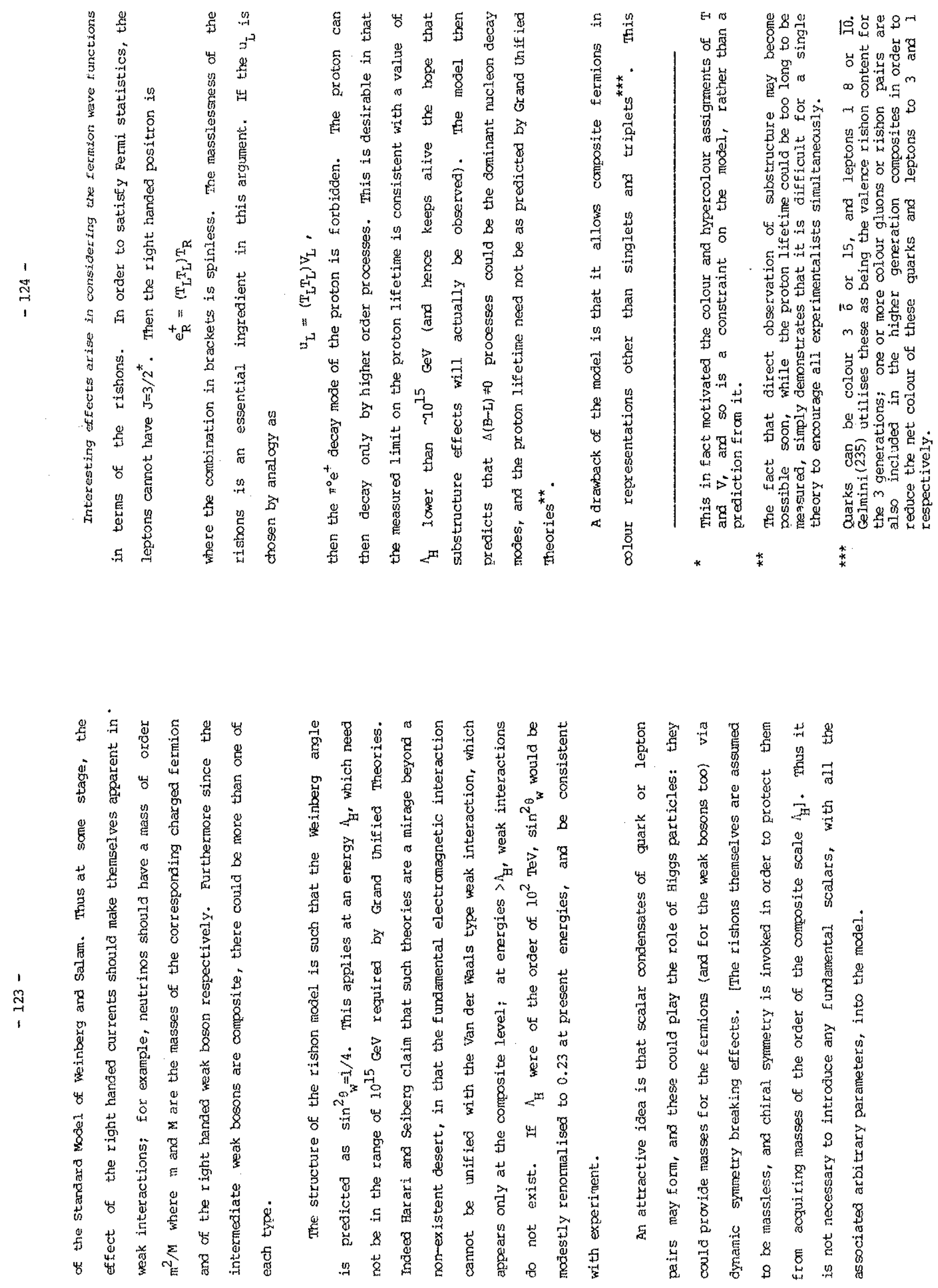

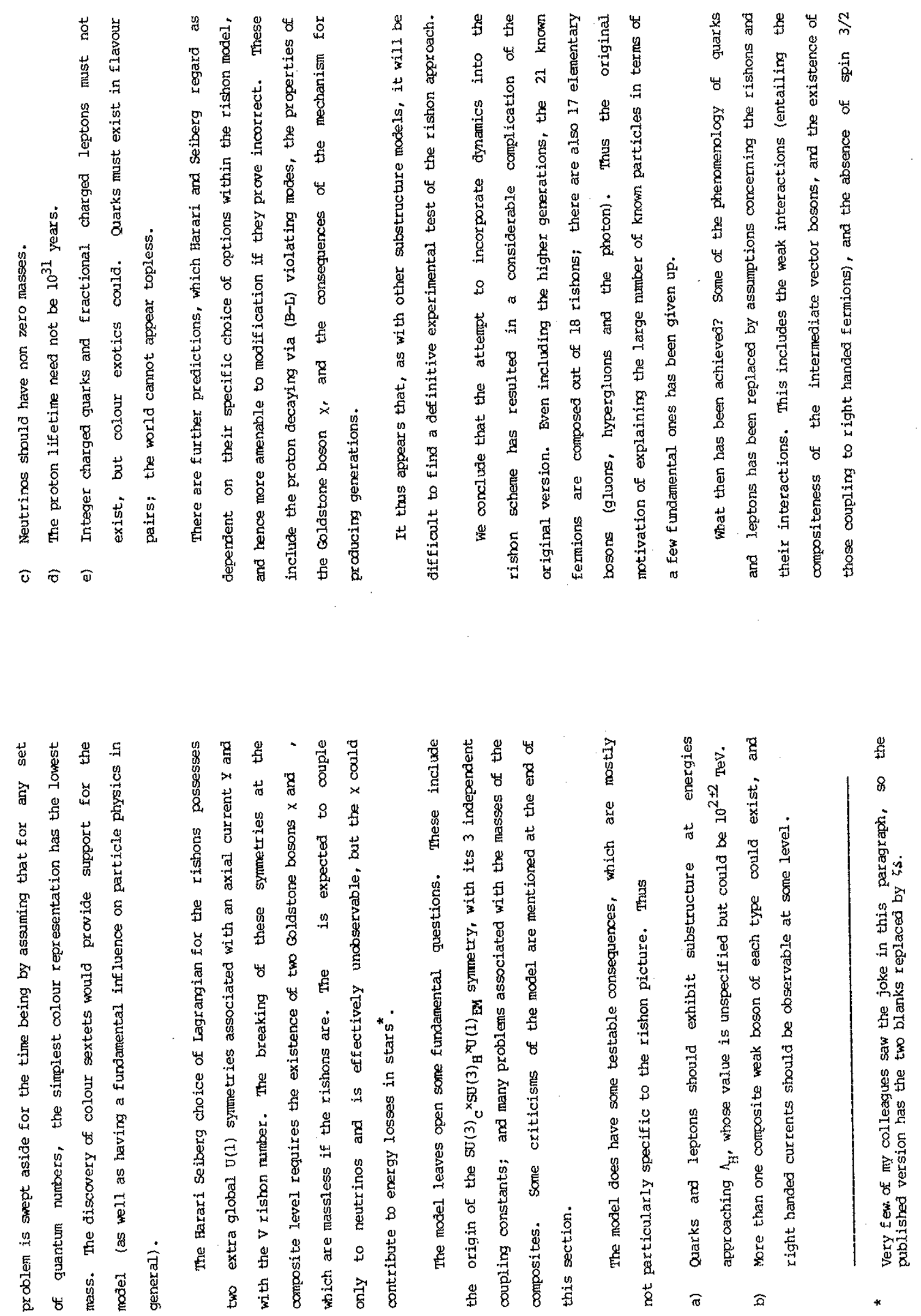


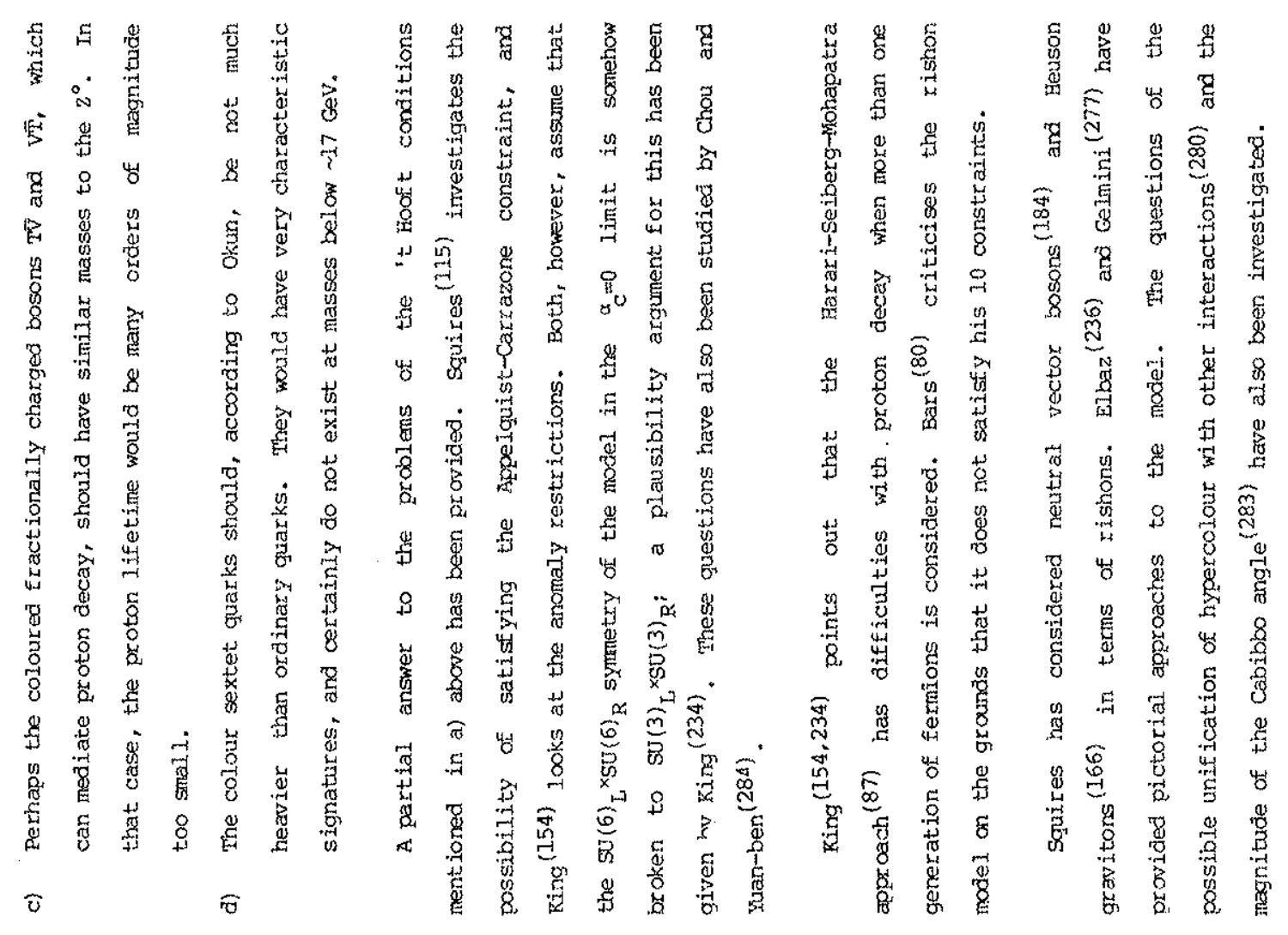

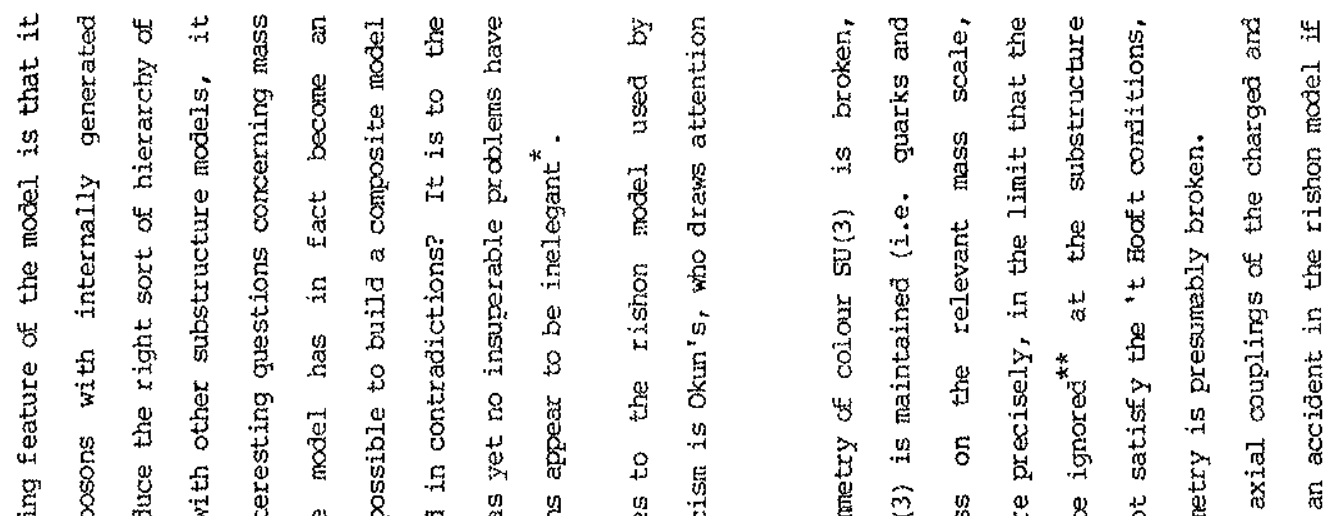

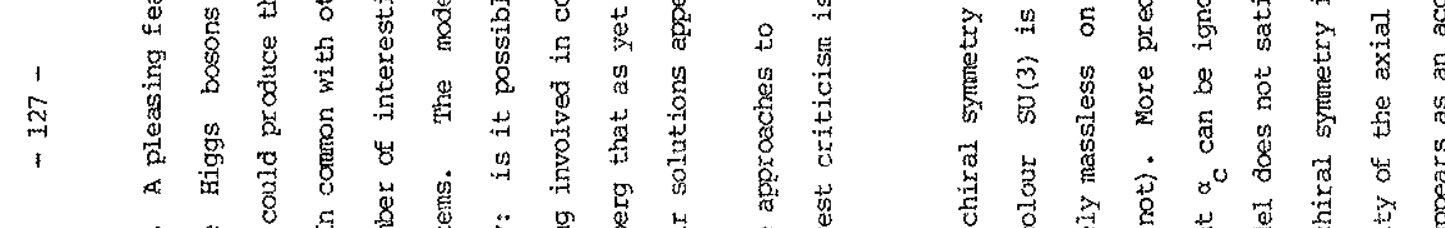

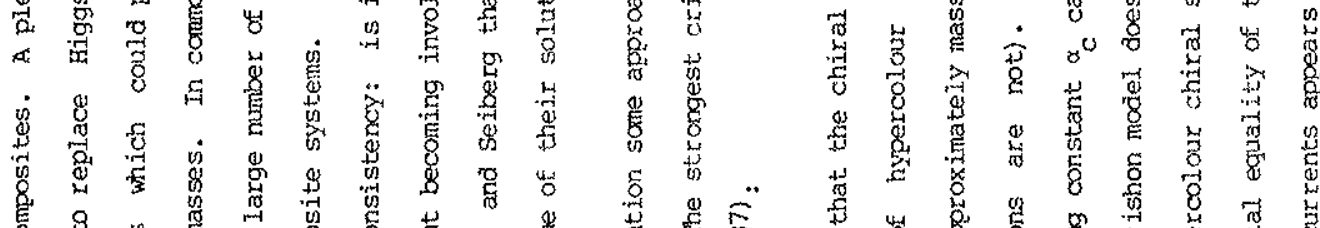

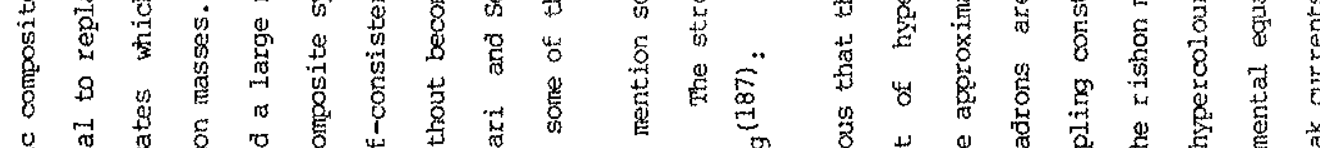

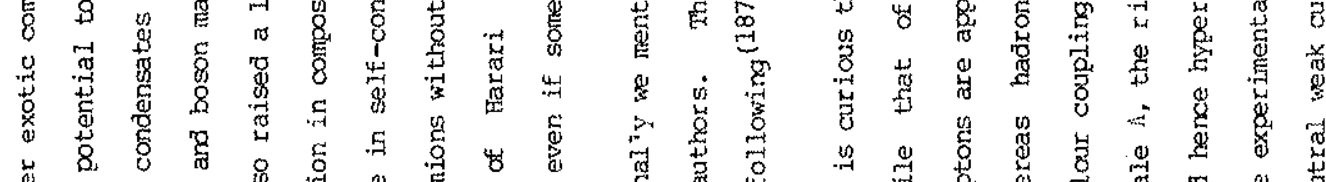

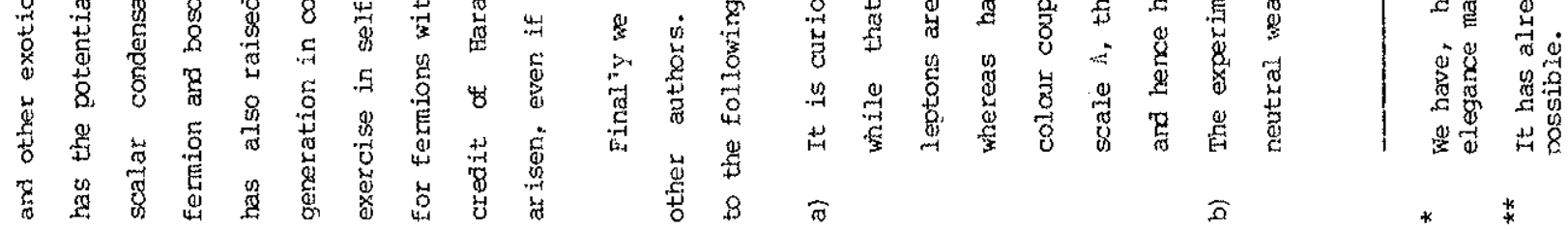



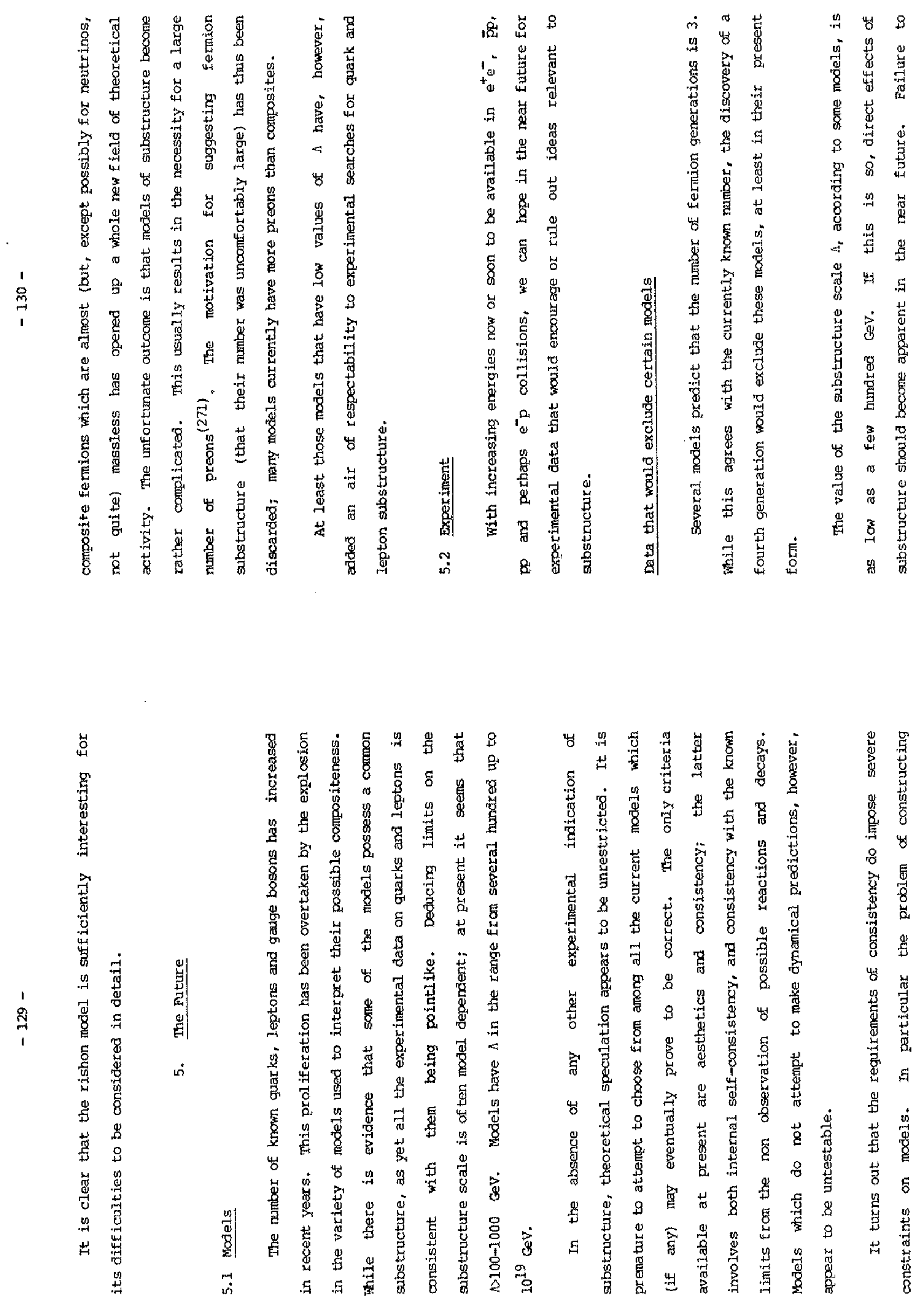

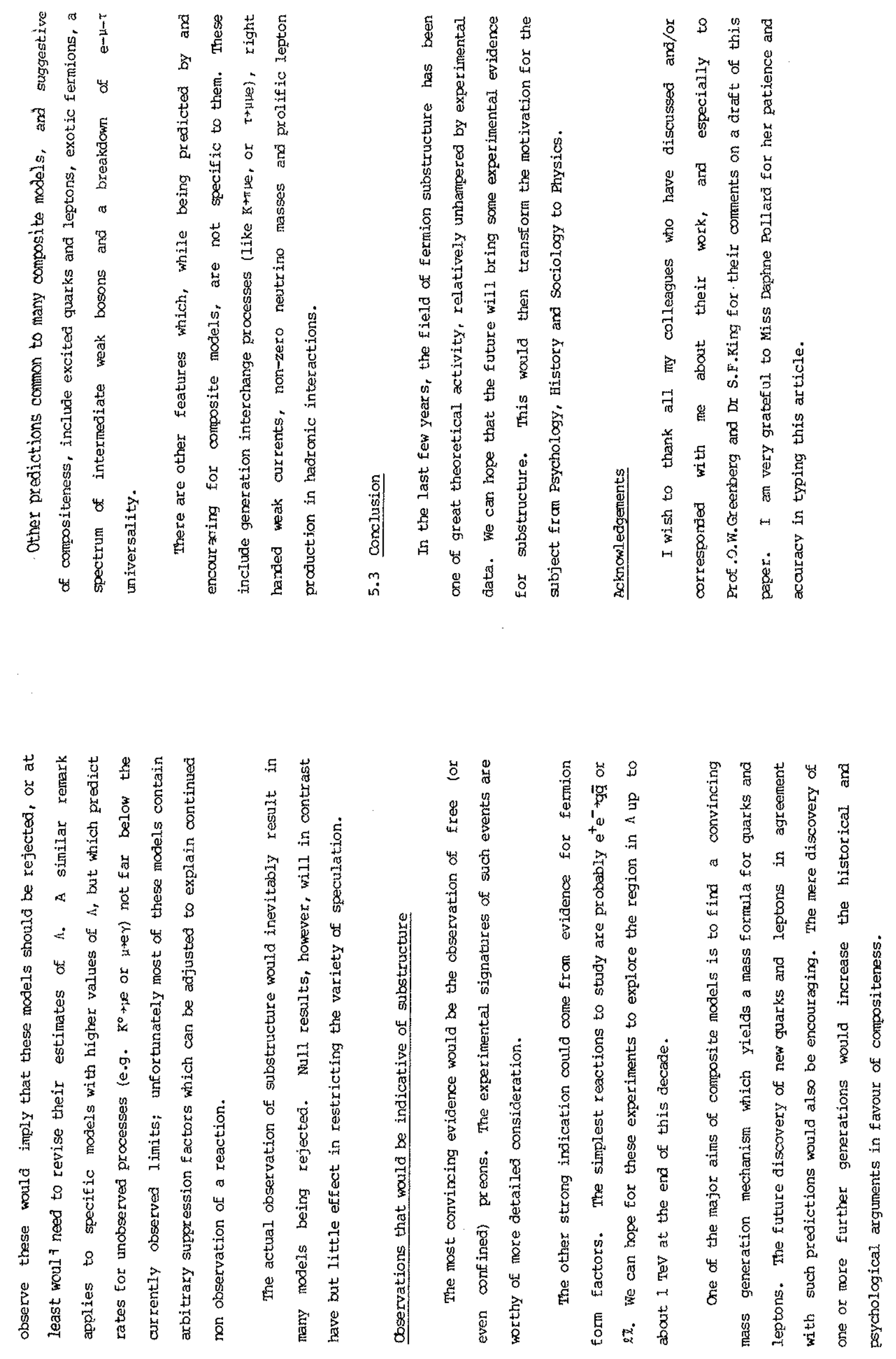


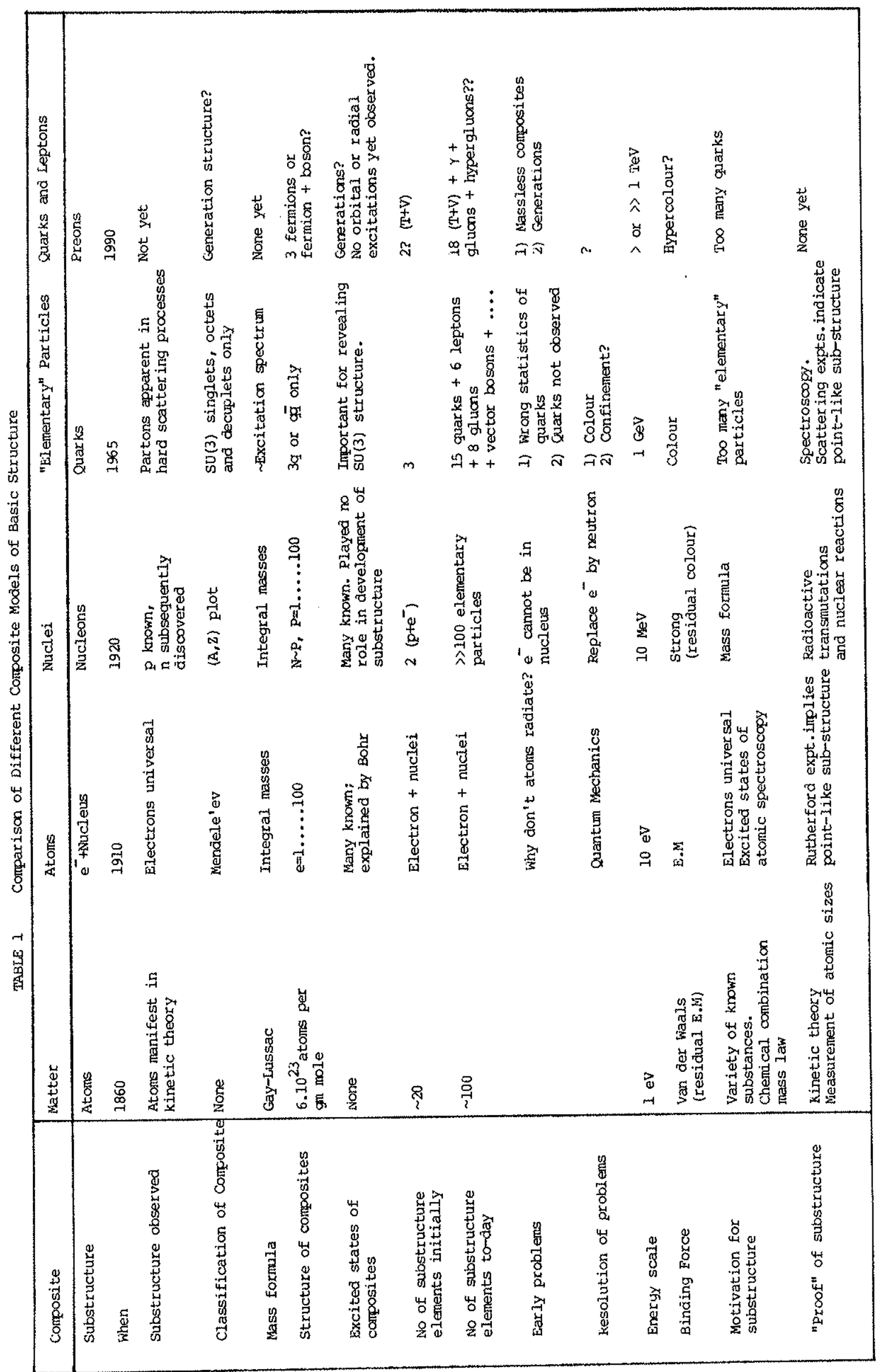



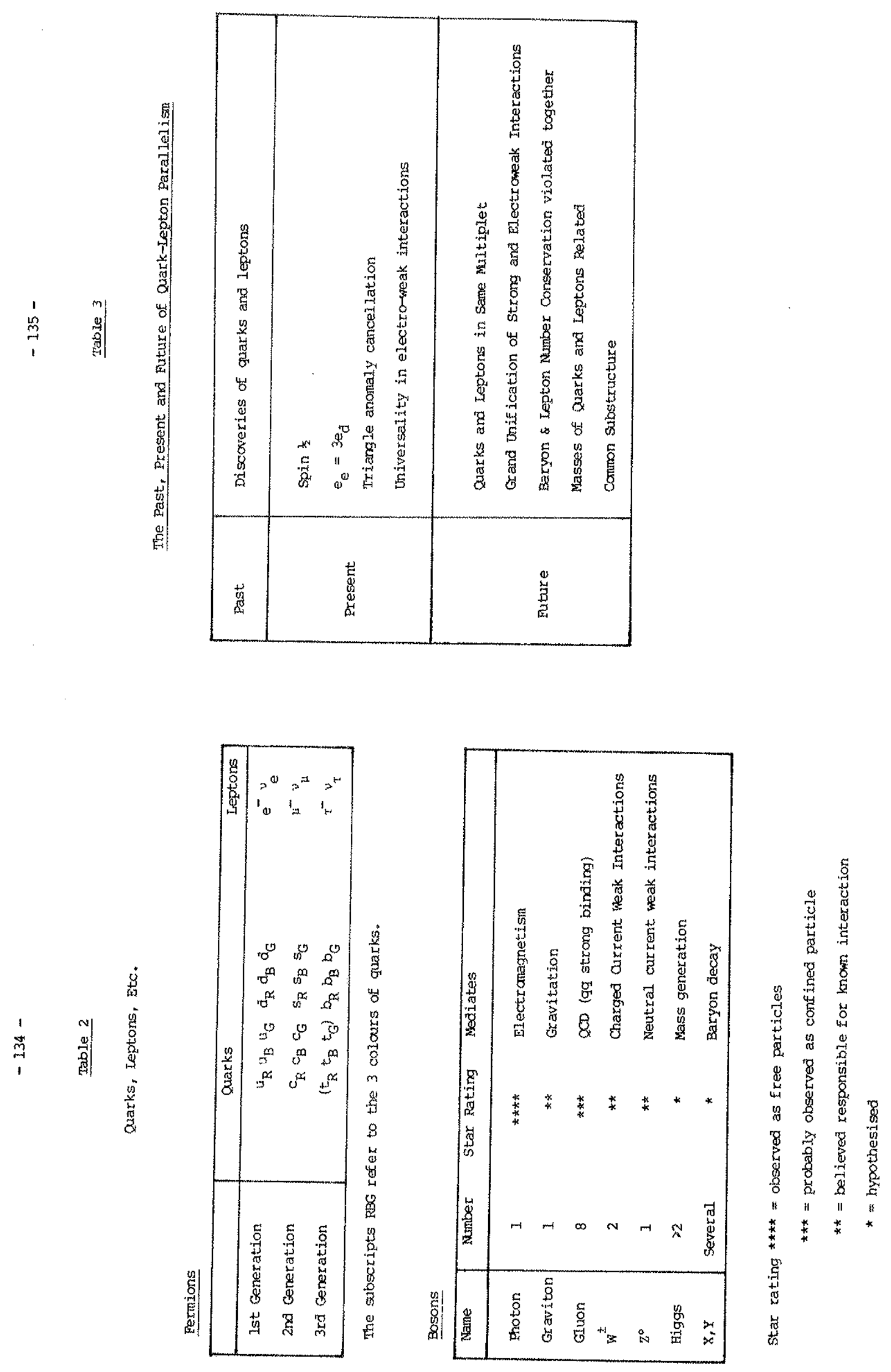


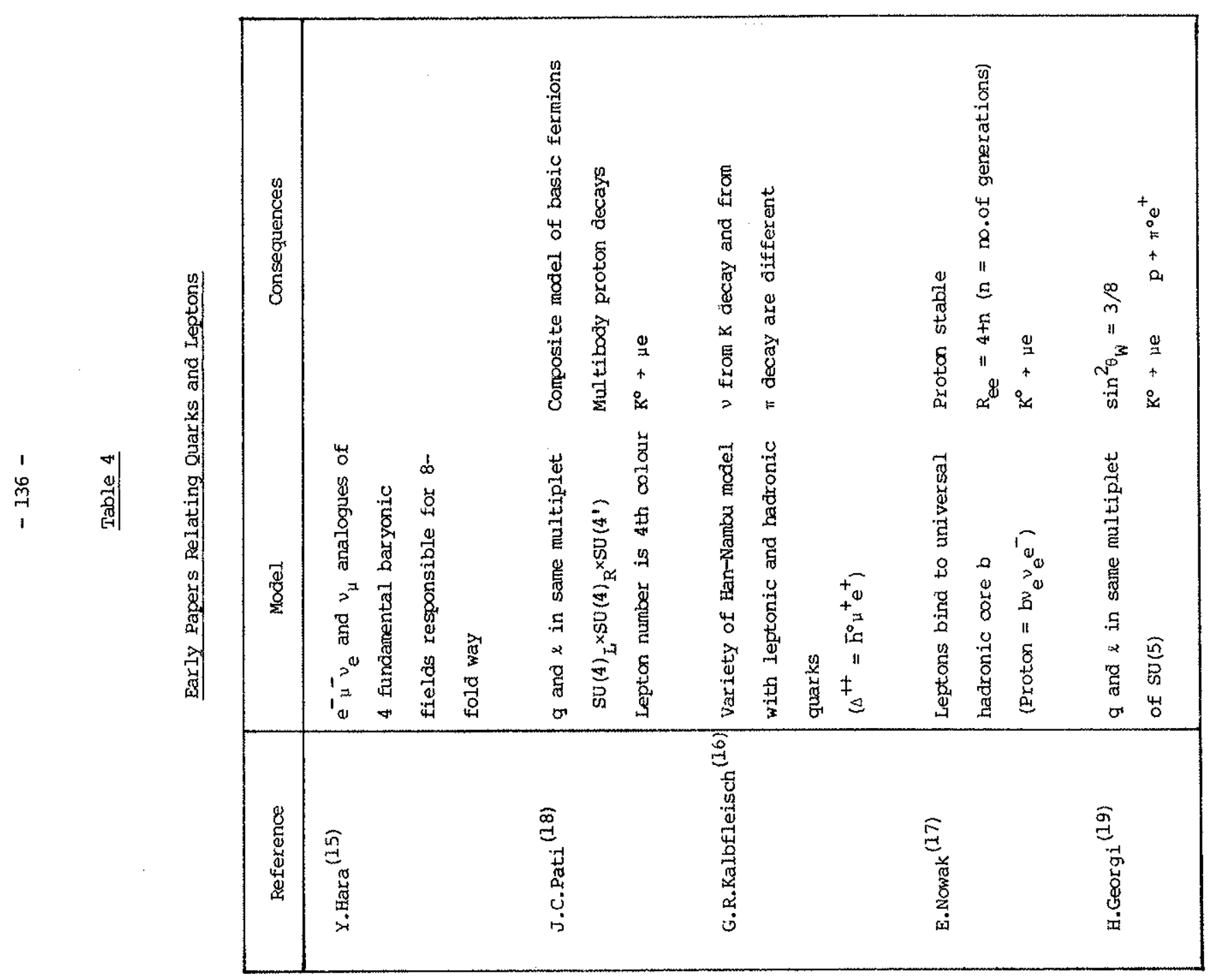




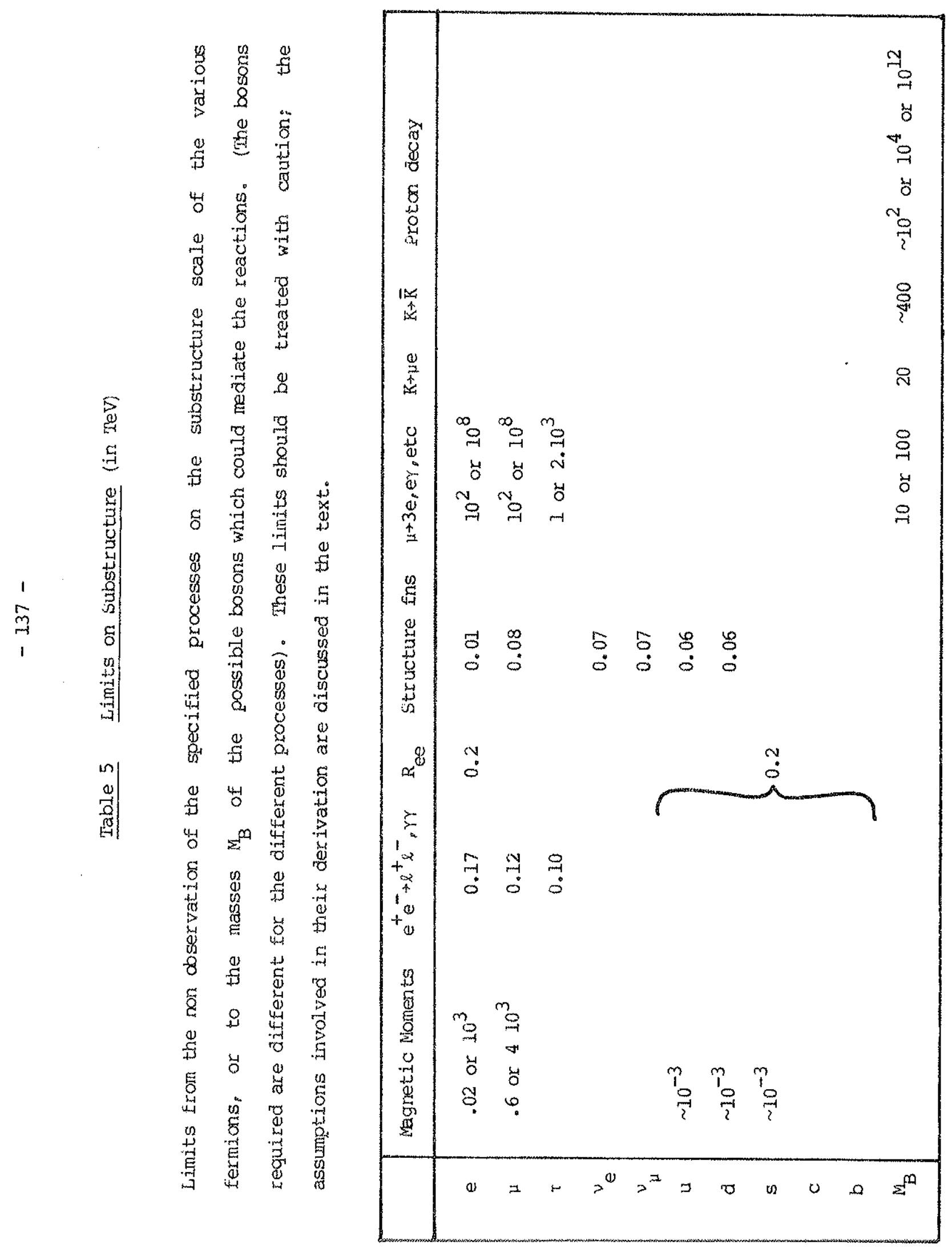


Table 6

(a) Values of MR for different levels of substructure

\begin{tabular}{|l|ccc|}
\hline Particle & M (GeV) & $R$ (cms) & MR \\
\hline Luarks and leptons & $\sim 1$ & $<10^{-16}$ & $<10^{-2}$ \\
Proton & 1 & $10^{-13}$ & 5 \\
Nucleus A & $\mathrm{A}$ & $\mathrm{A}^{1 / 3} 10^{-13}$ & $5 \mathrm{~A}^{4 / 3}$ \\
Hyörogen atom & 1 & $10^{-8}$ & $10^{5}$ \\
Positronium & $10^{-3}$ & $10^{-8}$ & $10^{3}$ \\
\hline
\end{tabular}

(b) Upper limits on MR for quarks and leptons

\begin{tabular}{|c|c|c|c|c|}
\hline Farticle & $M(\mathrm{GeV})$ & $\begin{array}{l}\text { Lower limit on } \\
\mathrm{R}^{-1}(\mathrm{TeV})\end{array}$ & $\mathrm{MR}_{1}^{*}$ & $\mathrm{MR}_{2}^{*}$ \\
\hline e & $0.510^{-3}$ & $0.2^{* *}$ & $210^{-6}$ & $10^{-6}$ \\
\hline$\nu_{e}$ & $<0.510^{-7}$ & $0.07^{+}$ & $10^{-9}$ & $10^{-1.0}$ \\
\hline$\mu$ & $10^{-1}$ & $0.6^{t+}$ & $210^{-4}$ & $210^{-4}$ \\
\hline$\nu_{\mu}$ & $<0.610^{-3}$ & $0.07^{+}$ & $10^{-5}$ & $10^{-6}$ \\
\hline$\tau$ & 1.8 & $0.1^{* *}$ & $210^{-2}$ & $310^{-3}$ \\
\hline$\nu_{\tau}$ & $<0.3$ & - & - & $510^{-4}$ \\
\hline $\mathrm{u}$ & 0.3 & $0.2^{* *}$ & $10^{-3}$ & $510^{-4}$ \\
\hline d & 0.3 & $0.2^{\star \star}$ & $10^{-3}$ & $510^{-4}$ \\
\hline s & 0.5 & $0.2^{* *}$ & $10^{-3}$ & $10^{-3}$ \\
\hline c & 1.5 & $0.2^{\star \star}$ & $10^{-2}$ & $310^{-3}$ \\
\hline b & 5 & $0.06^{* *}$ & $10^{-1}$ & $10^{-2}$ \\
\hline
\end{tabular}

* $M R_{1}$ is for individual limits on $R ; M R_{2}$ for a common $R^{-1}>0.6 \mathrm{I}^{\mathrm{e}} \mathrm{eV}$

** From $e^{+} \mathrm{e}^{--}$reactions

+ From $v N$ reactions, interpreted in terms of a $v$ charged radius or a form factor

+ From $9-2$, interpreted as $O(M / M)^{2}$ 


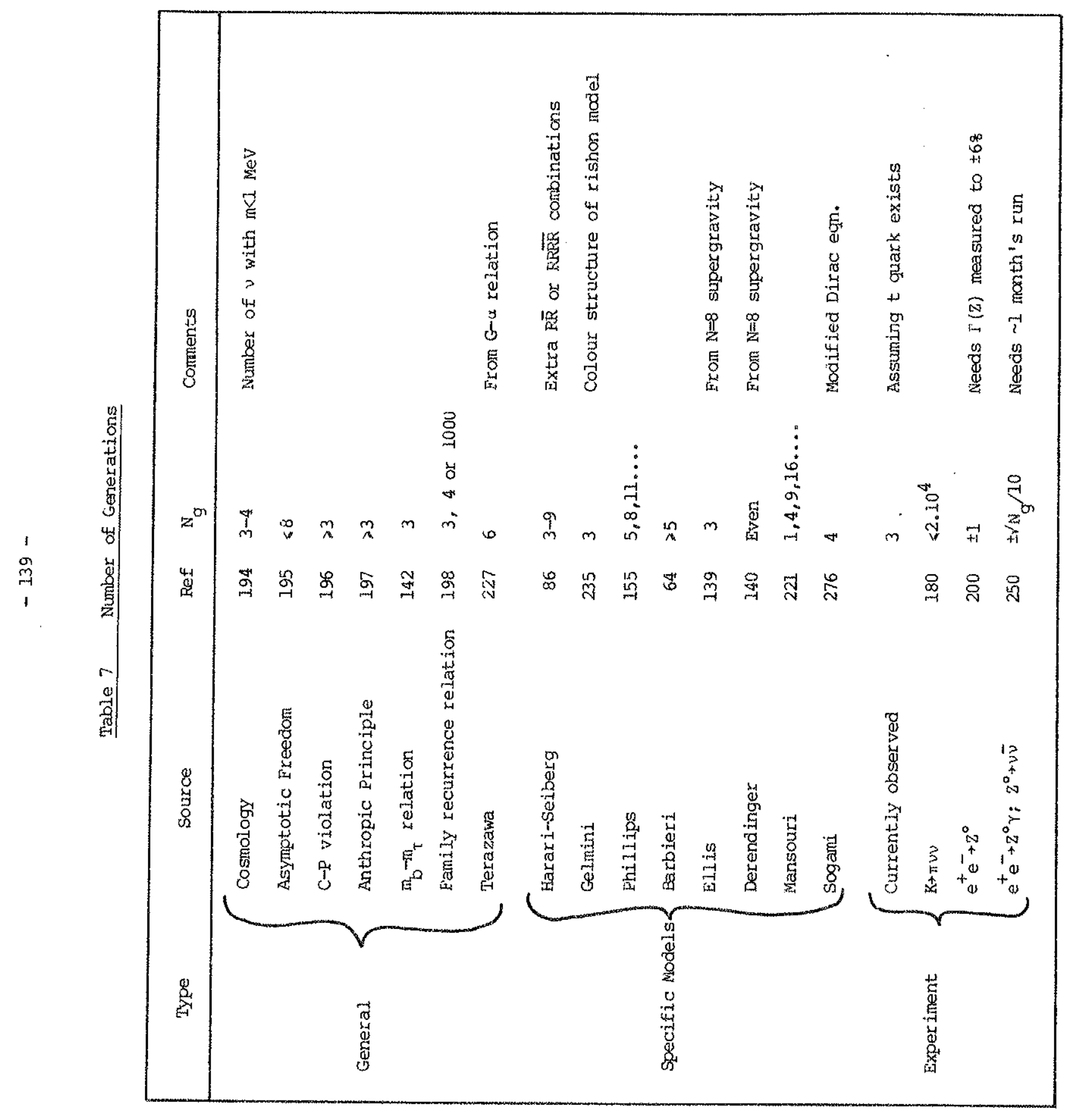


TABLE 8

Choice of generation number for antiparticles

\begin{tabular}{|c|c|c|c|}
\hline Reaction & $G\left(e^{\psi}\right)=+1$ & $G\left(e^{+}\right)=-1$ & Exper iment \\
\hline$e^{+} e^{-m}+\mu^{+} \mu^{-}$ & Forbidden & Allowed & $\begin{array}{l}\text { Copious. Similar to } \\
e^{+} e^{-} \cdot e^{+} e^{-}\end{array}$ \\
\hline$e^{--} e^{-}+\mu-\cdots$ & Allowed & Forbidden & Not seen \\
\hline$d \ddot{d}+s^{*}$ & Forbidden & Allowed & $\begin{array}{l}\text { Copious. Similar to } \\
d \vec{a}+u \bar{u}\end{array}$ \\
\hline$\alpha \mathrm{dar}+\mathrm{ss}^{* *}$ & Allowed & Forbidden & Not seen \\
\hline
\end{tabular}

* For example, associated production of strange particle pairs in hadronj.c collisions.

** For example, $\pi^{-} \mathrm{p}^{+\Sigma^{+} \mathrm{K}^{-}}, \mathrm{pp} \rightarrow \Sigma^{+} \mathrm{\Sigma}^{+}, \bar{\Sigma}^{-}+\mathrm{n} \mathrm{r}^{-}$ 
TABLE 9

Unusual Reactions Conserving Generation Number

The table 1 ists reactions which conserve baryon and lepton numbers. Reactions wich are allowed by conventional flavour selection rules are not included.

A) Reactions in which each generation number is separately conserved.

\begin{tabular}{|c|c|c|}
\hline Allowed & Expeximental Limit & Forbididen \\
\hline$\mu^{m} \mathrm{~d} r e^{-m} s$ & & $1^{t} \mathrm{~d}+\mathrm{e}^{+} \mathrm{s}$ \\
\hline$e^{+} a \cdot r \mu^{+} s$ & & $e^{-} d^{-} \mu_{1}^{-} s$ \\
\hline$v_{\mu} \mathrm{d}+\mathrm{e}^{-c} \mathrm{c}$ & $\mathrm{e}^{-\mathrm{c} / 1}-\mathrm{x}<0.68(209,210)$ & $\tilde{v}_{n} u+e^{+} s$ \\
\hline $\bar{v}_{e^{u}+\mu^{+} s}$ & & $v^{a+1 \mu^{-m} s}$ \\
\hline$K_{C}^{0} \rightarrow H^{ \pm} e^{5}$ & $\mu \mathrm{e} / \mu \mu<0.2$ or $700(76,77)$ & \\
\hline$k^{+}+11^{+} v_{e}$ & $\mu^{+} v_{\mathrm{e}} / \mu^{+} v_{\mu}<0.6800$ & $x^{+}+e^{-t} v_{\mu}$ \\
\hline $\mathrm{k}^{+} \rightarrow \pi^{+} \mathrm{j}^{+} \mathrm{e}^{-}$ & $\pi^{+} u^{+} e^{-} / n^{+} e^{+} e^{-}<28(74)$ & $\mathrm{k}^{+}+n^{+} \mathrm{w}^{-} \mathrm{e}^{+}$ \\
\hline$\Sigma^{+}+p^{+} \mu^{-}$ & & $\mathrm{r}^{+}+\mathrm{pu}^{+} \mathrm{e}^{-}$ \\
\hline$b+d \tau-e^{+}$ & & $b+d r^{+} e^{--}$ \\
\hline
\end{tabular}

B) Reactions in which net generation number is conserved, assuming that generation number assignnents satisfy $\mathrm{G}_{3}-\mathrm{G}_{2}=\mathrm{G}_{2} \mathrm{G}_{\mathrm{L}} \mathrm{L}$

\begin{tabular}{|c|c|}
\hline Allowed & Forbidden \\
\hline$r^{+}+K^{0} \mu^{+} \quad \tau^{+}+K^{0} \mu^{+} / 211<10^{-3(65)}$ & \\
\hline$\tau^{+}+\mu_{i j}^{+} e^{-}$ & $r^{+}+\mu^{+} \mu \mu^{-} e^{+}$ \\
\hline $\mathrm{r}^{+} \rightarrow \pi^{-} \mathrm{K}^{+} \mathrm{u}^{+}$ & $t^{+}+\pi^{+} K^{-} \mu^{+}$ \\
\hline $\mathrm{b}+\mathrm{c \mu}-\vec{v}_{\mathrm{e}}$ & \\
\hline $\mathrm{b}+\operatorname{ss} \overline{\mathrm{d}} \quad\left(\mathrm{e} \cdot \mathrm{g} \cdot \mathrm{B}^{\circ}+\overline{\mathrm{K}}^{\circ} \mathrm{K}^{\circ}{ }^{*}\right)$ & $b+\operatorname{sis} \alpha\left(e . g \cdot B^{0}+K^{+} K^{-x}\right)$ \\
\hline$b \rightarrow \operatorname{csu} \quad\left(e \cdot g \cdot B^{-m}+K^{-*} D^{\circ}\right)$ & $b+\ddot{c} s u\left(e . g \cdot B^{-m}+K^{-* *} \tilde{D}^{o}\right)$ \\
\hline $\mathrm{b}+\operatorname{cod}$ tūu $\left(e .9 \cdot \mathrm{B}^{\circ}+\mathrm{D}^{\circ} \mathrm{D}^{\circ}\right)$ & $b^{+}+\overline{C d}\left(e . g . B^{-} \rightarrow D^{\circ} D^{-*}\right)$ \\
\hline
\end{tabular}

C) Reactions in which net generation number is conserved modulo 3

\begin{tabular}{|c|c|}
\hline Allowed & Forbidden \\
\hline$e^{-} e^{-}+\mu^{-} t$ & $e^{*} e^{-}+p^{ \pm} \mathrm{r}^{\mp}$ \\
\hline$r^{m}+e^{-} e^{-}+$ & $r^{m}+e^{-2} e^{t} \mu^{-}$ \\
\hline$e^{-} d+\tau^{-n} s$ & $e^{t} d+r^{+} s$ \\
\hline $\bar{v}_{\mu} u \leftrightarrow e^{+} b$ & $v_{1} d \cdot e^{-} t$ \\
\hline
\end{tabular}



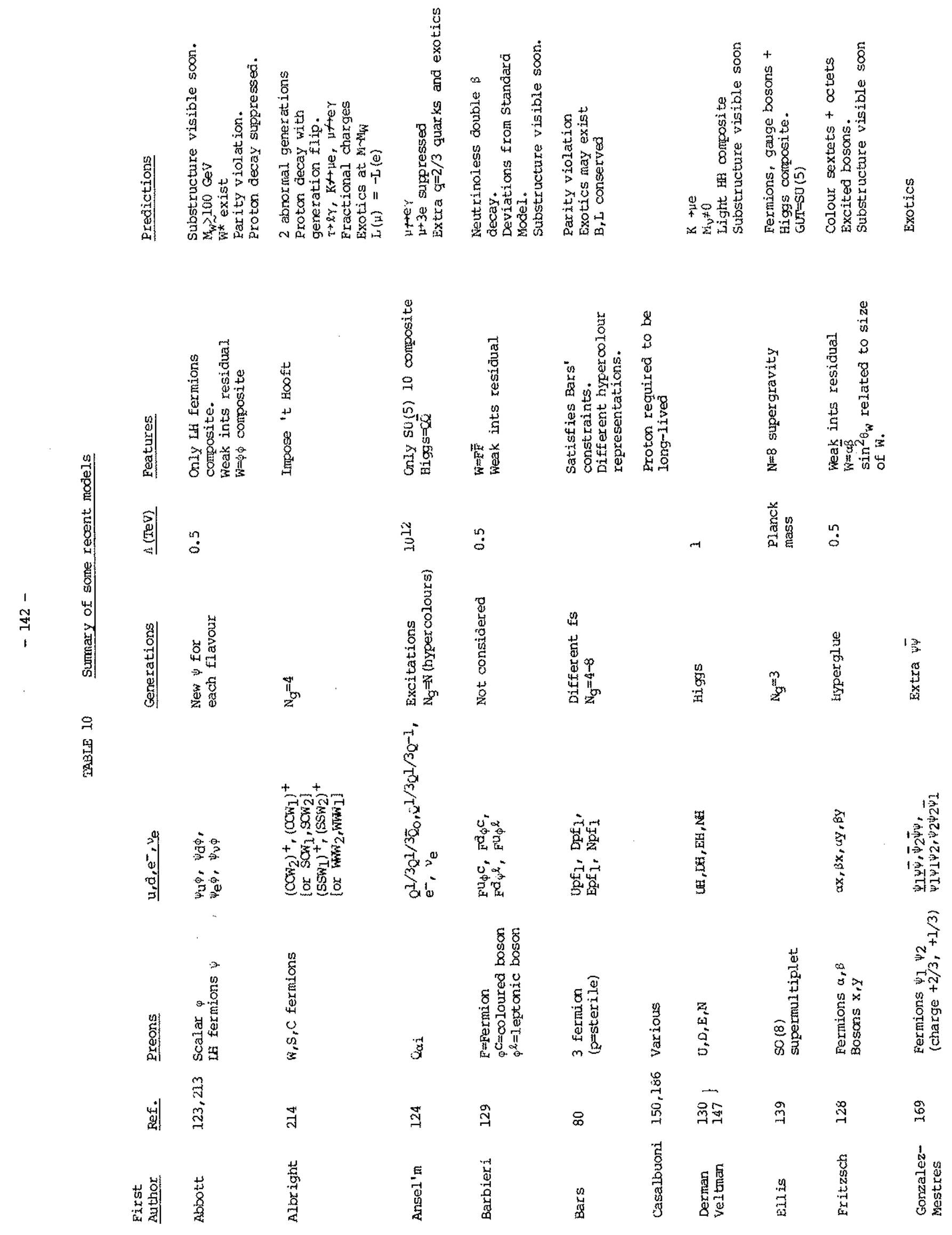

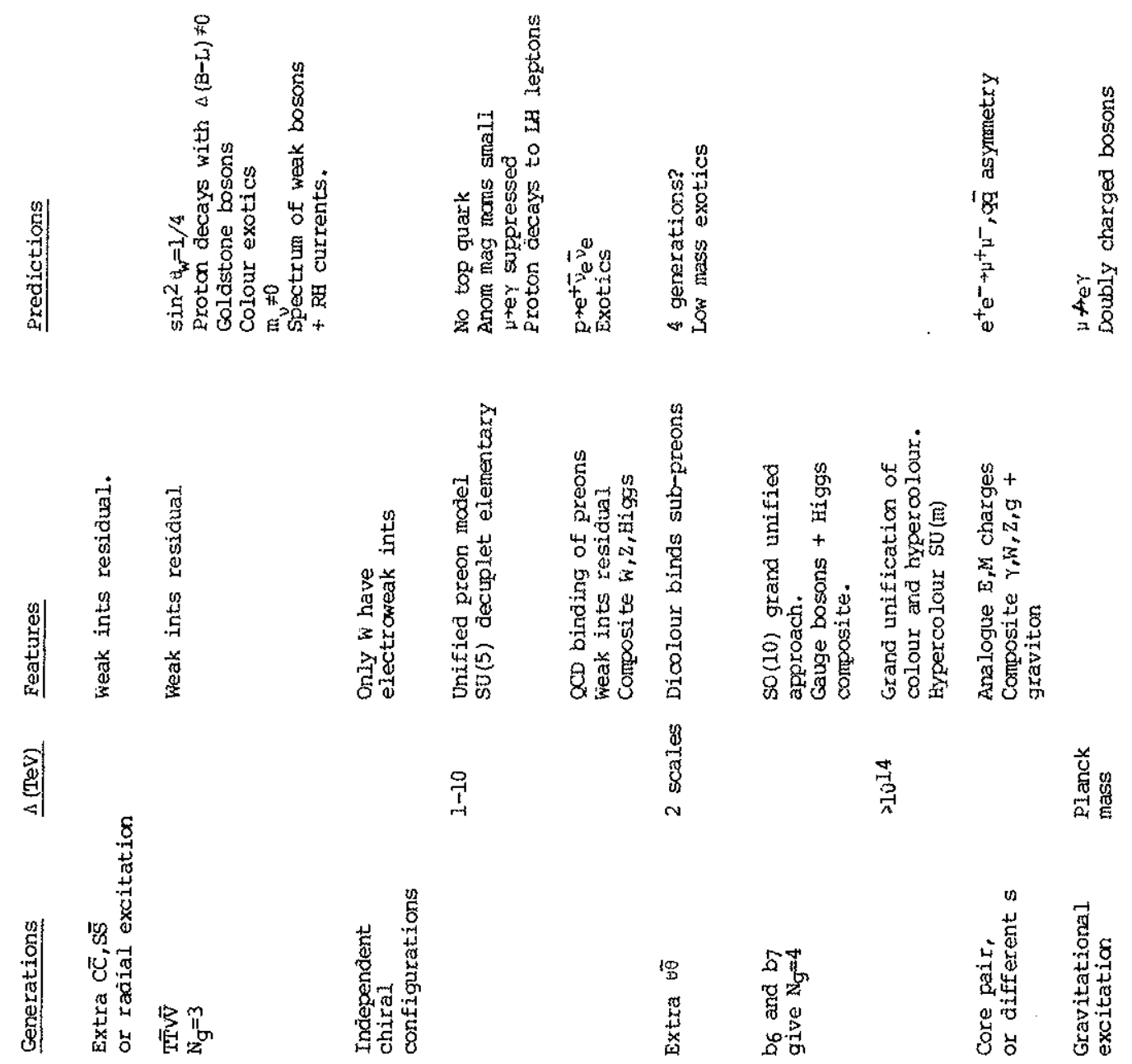

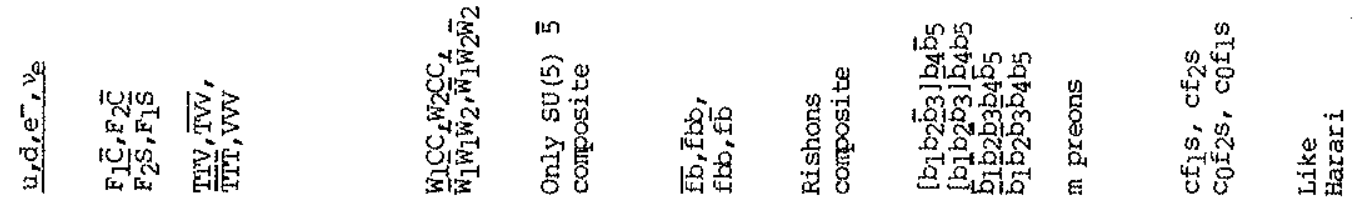

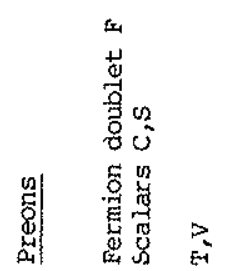

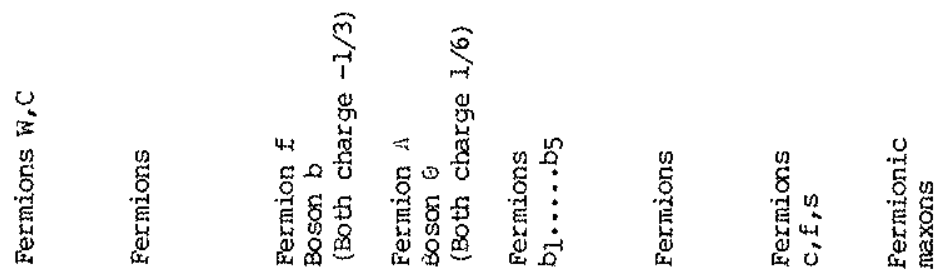

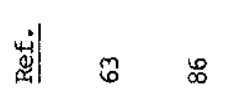
总

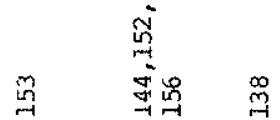

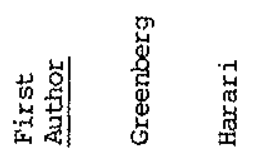

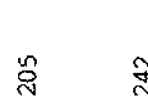

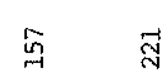

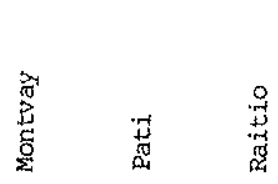




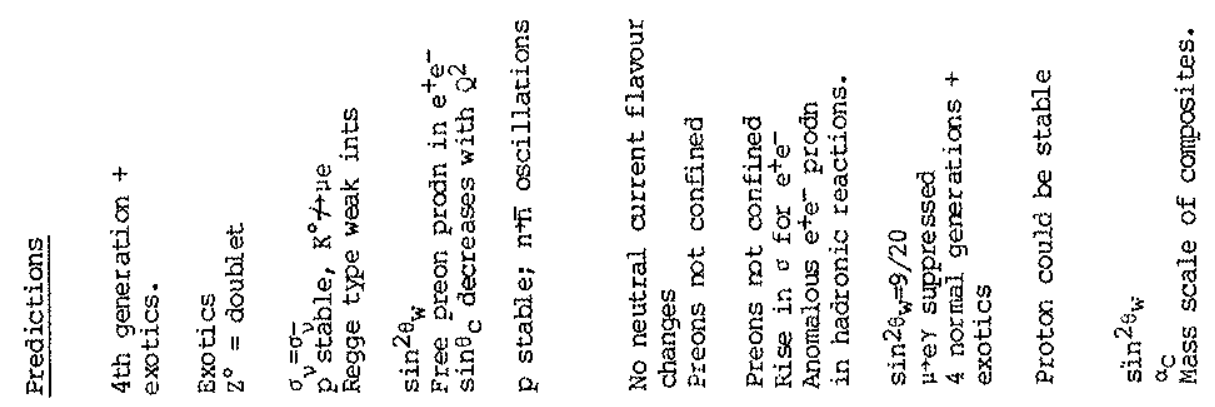

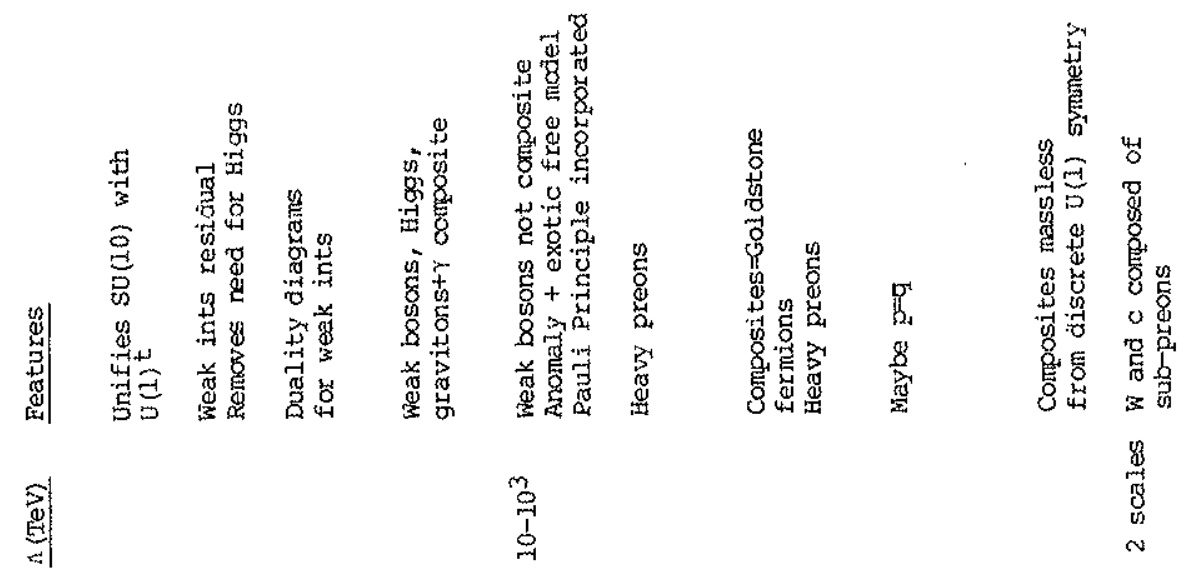

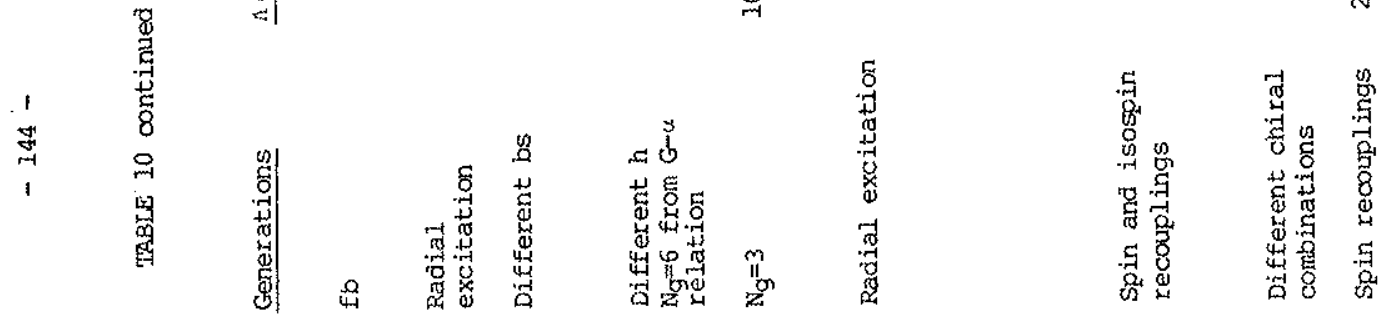

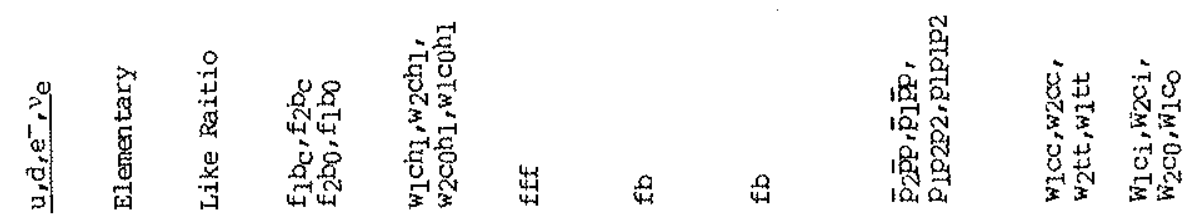

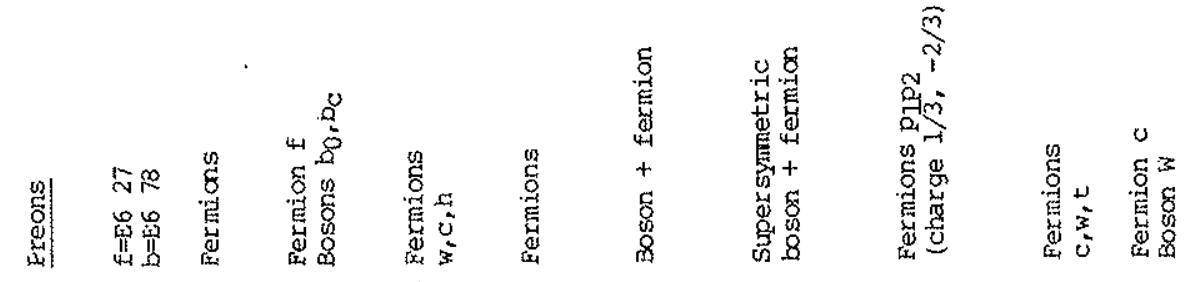

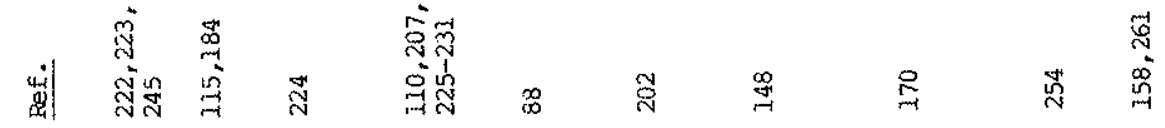

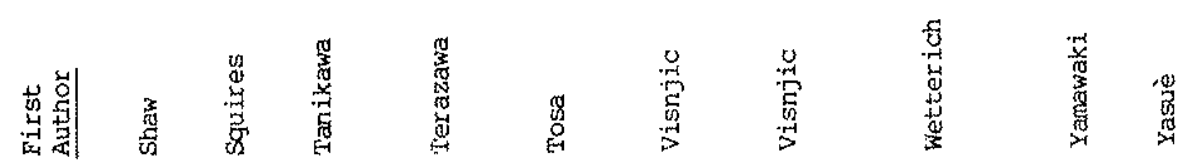



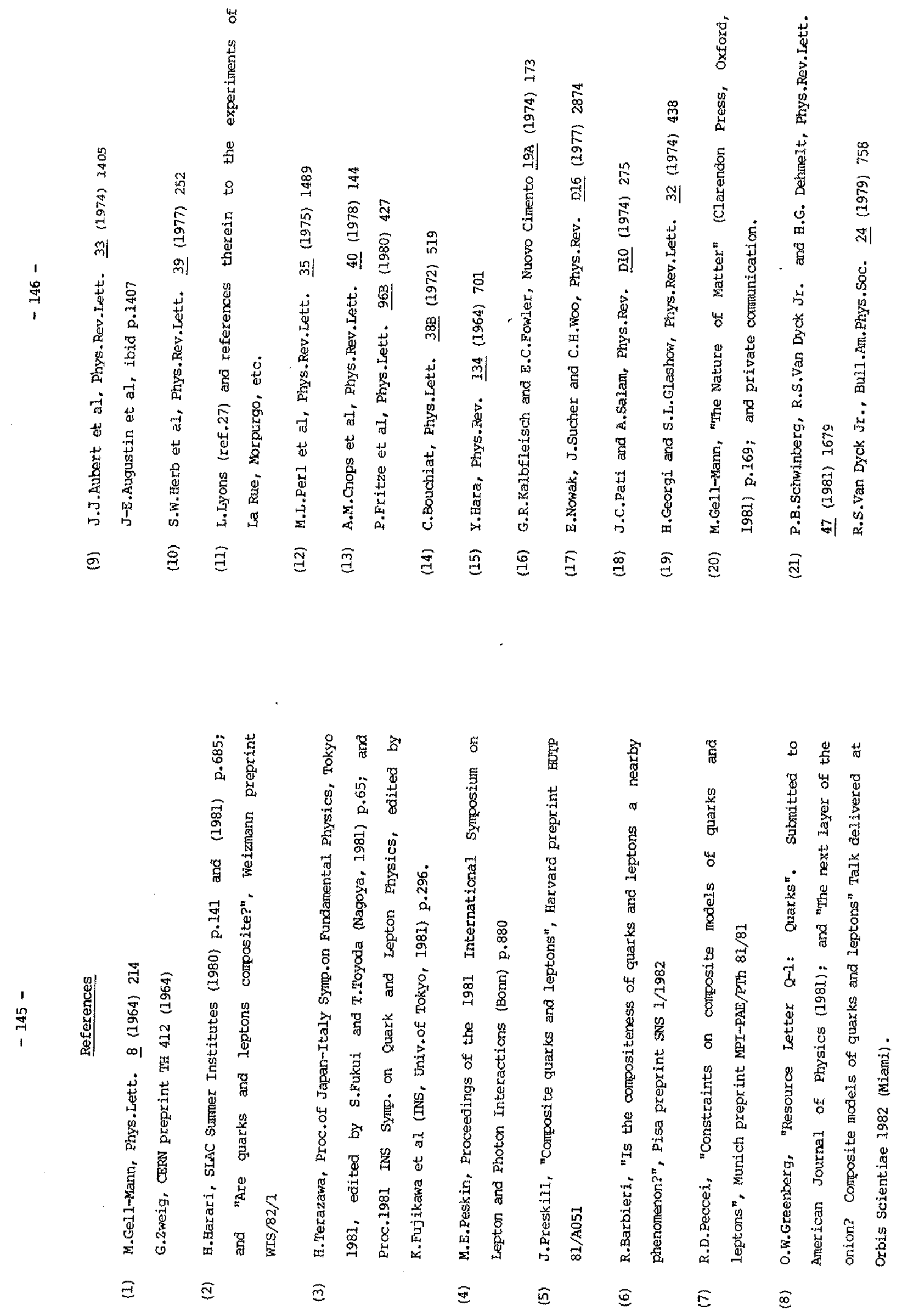

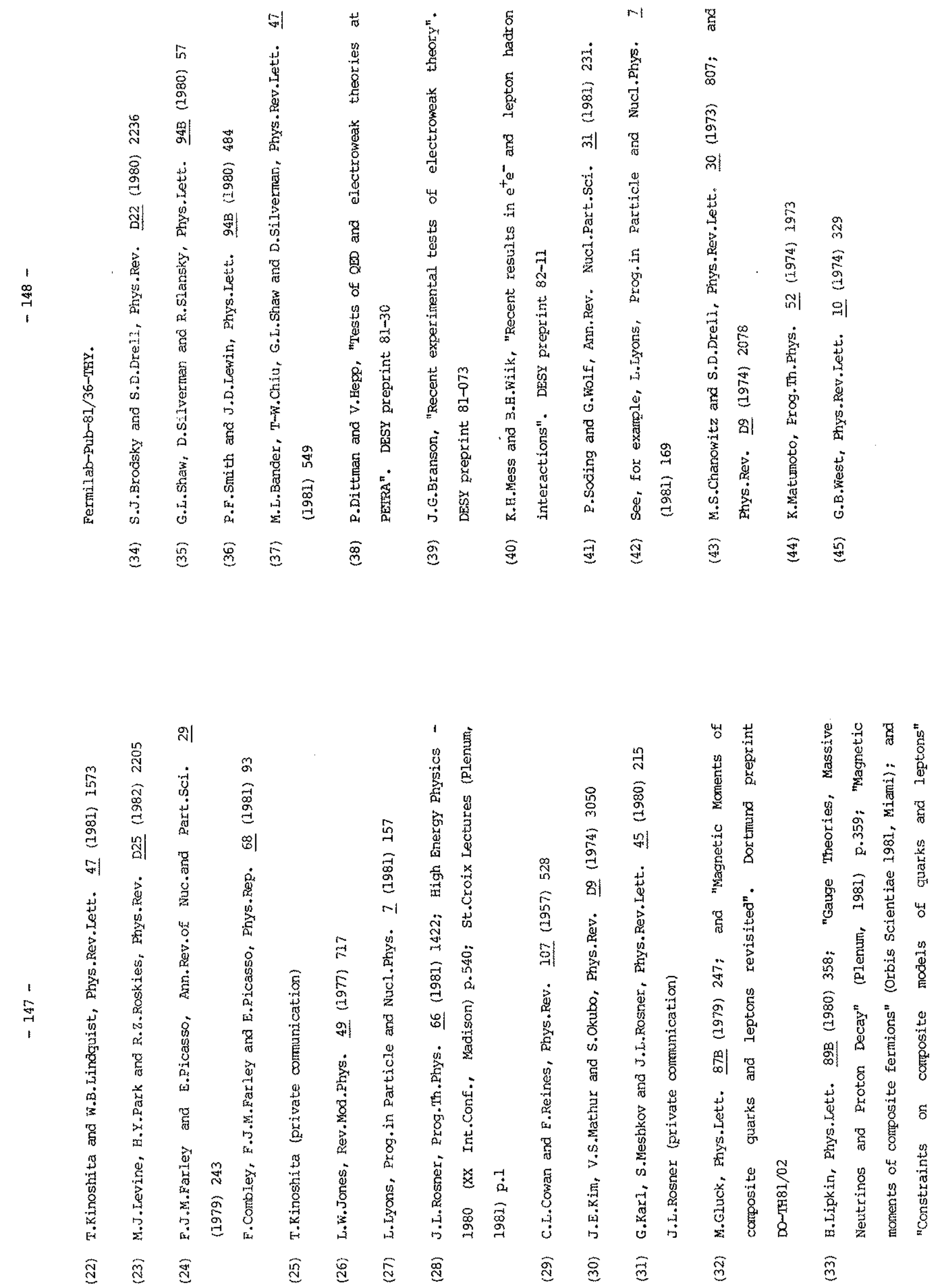


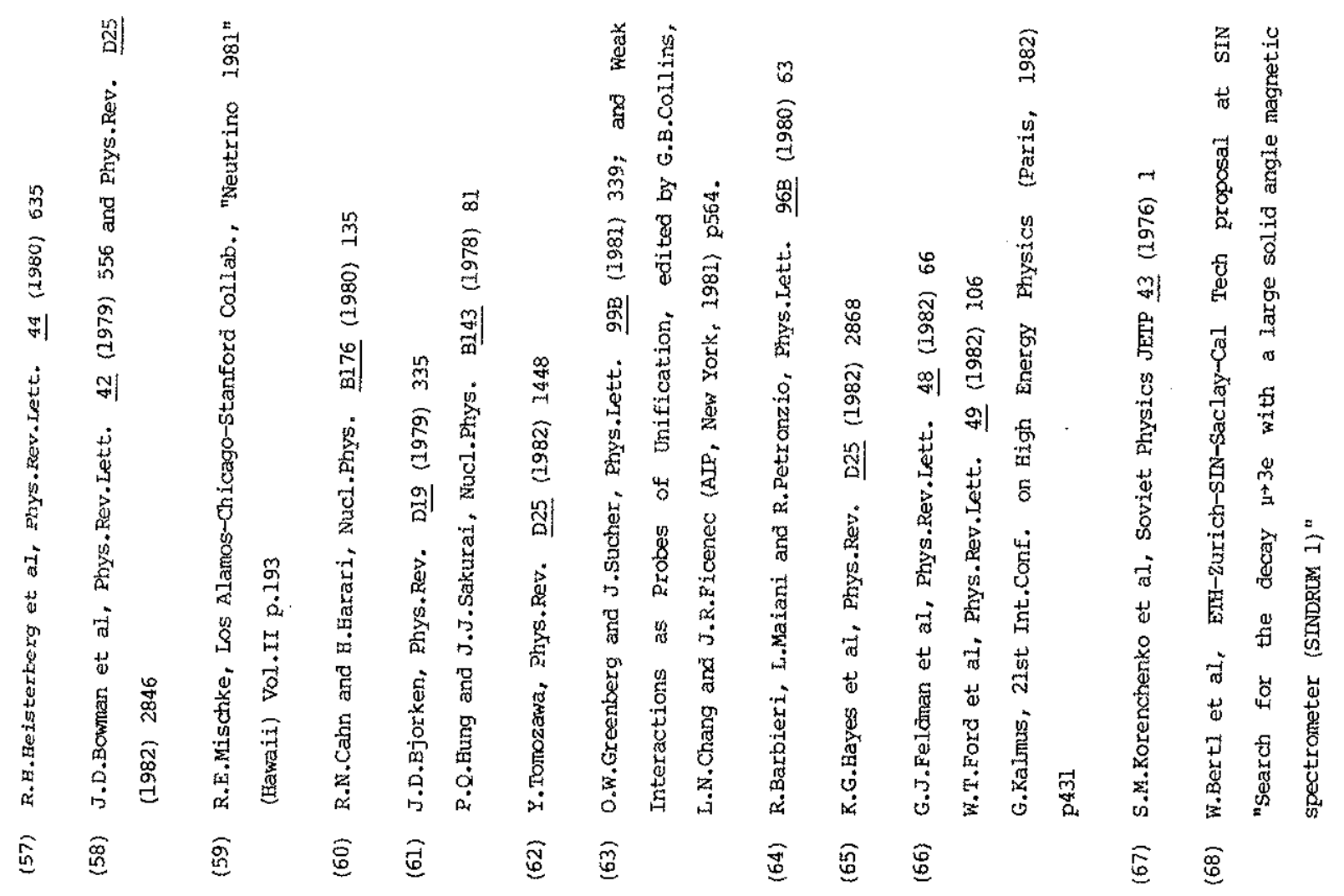

$\begin{array}{lll}\widehat{p} & \mathbb{E} & \widehat{D} \\ \stackrel{0}{g} & \stackrel{0}{g} & \stackrel{d}{d}\end{array}$

이 क्षा की

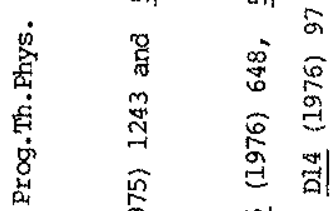

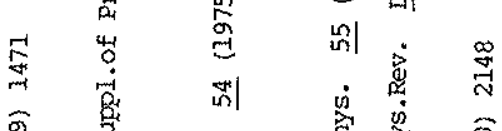

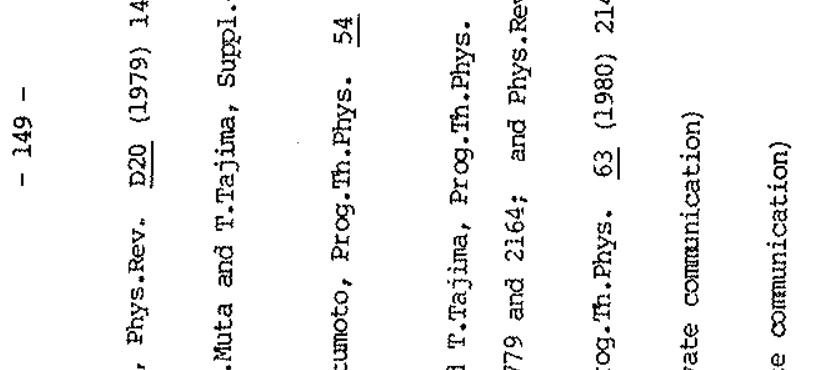

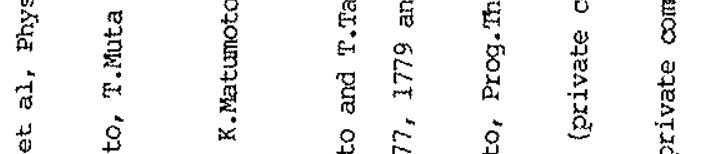

要是

要要总

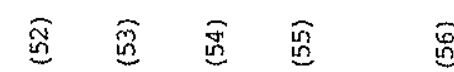




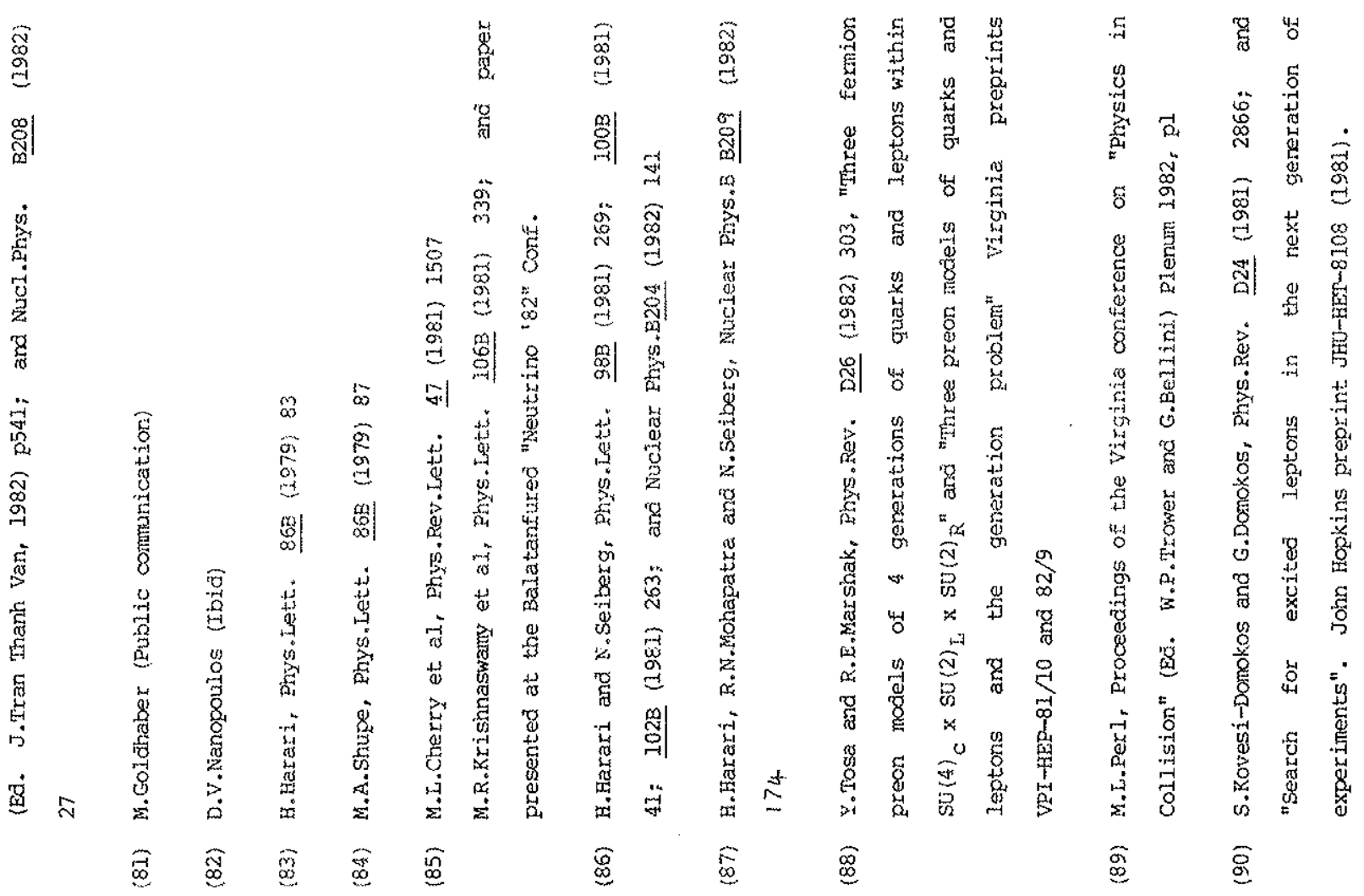

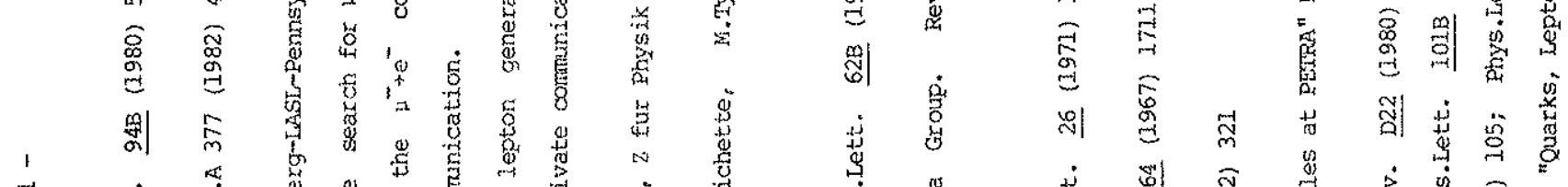

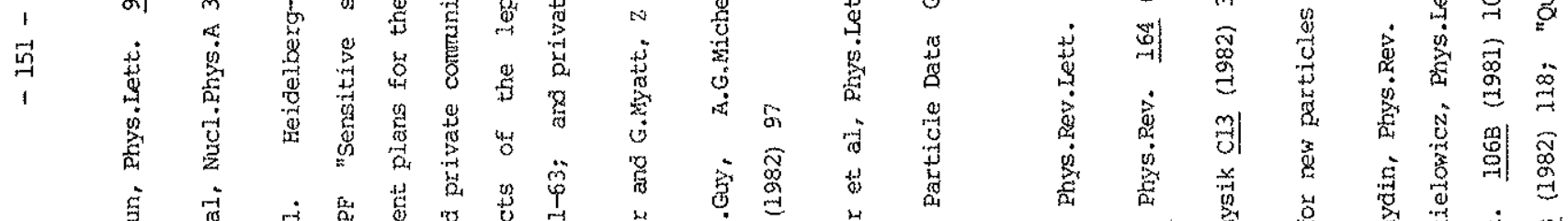

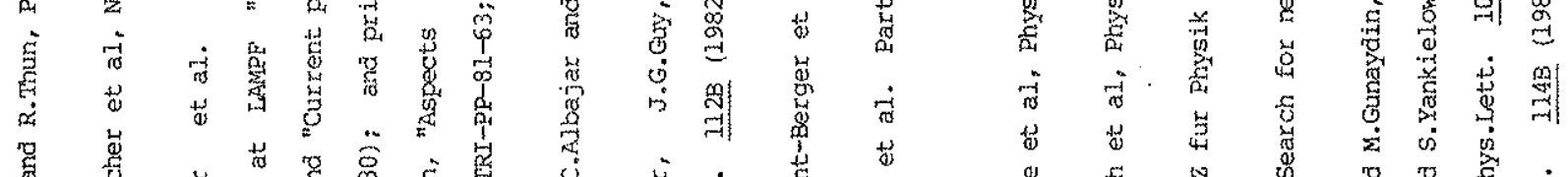

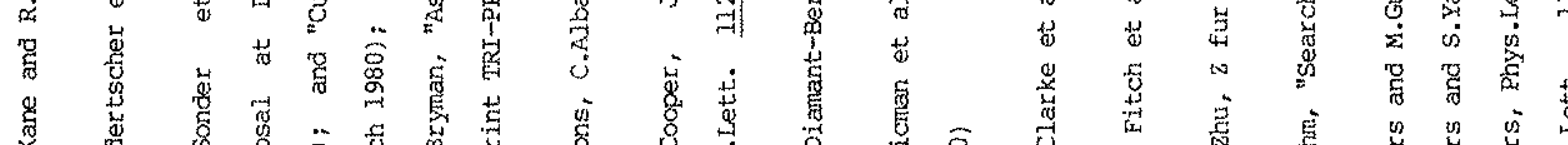

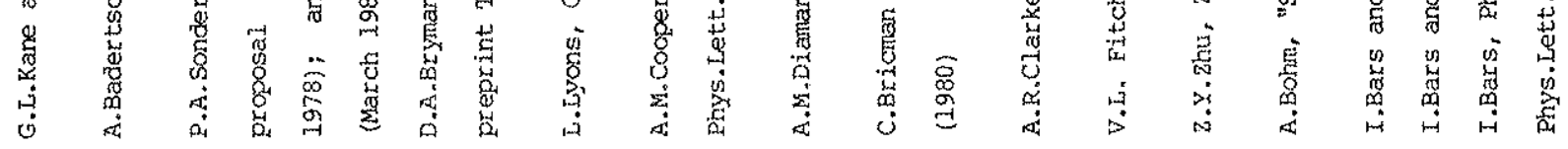

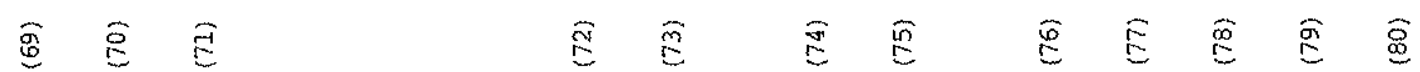



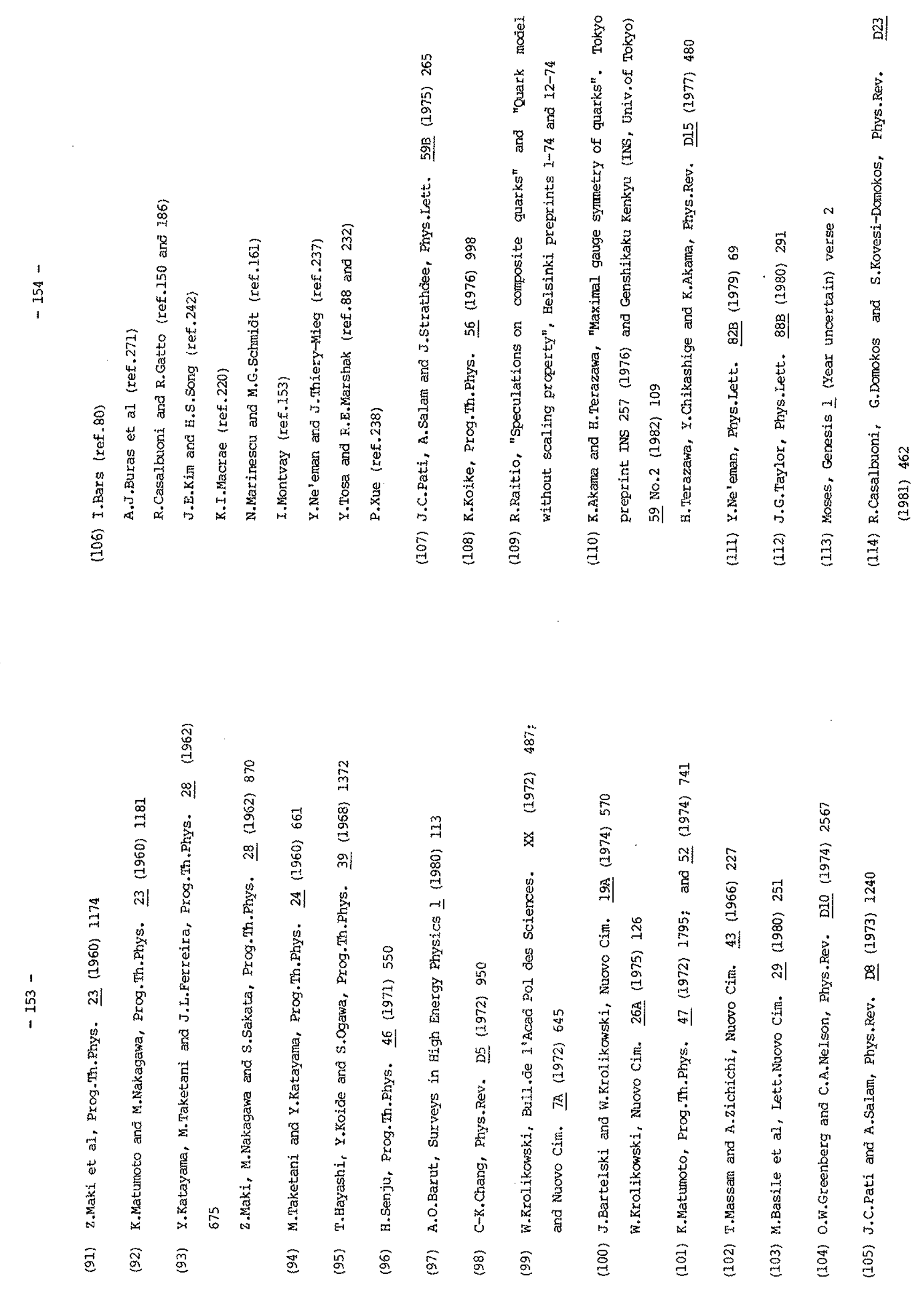

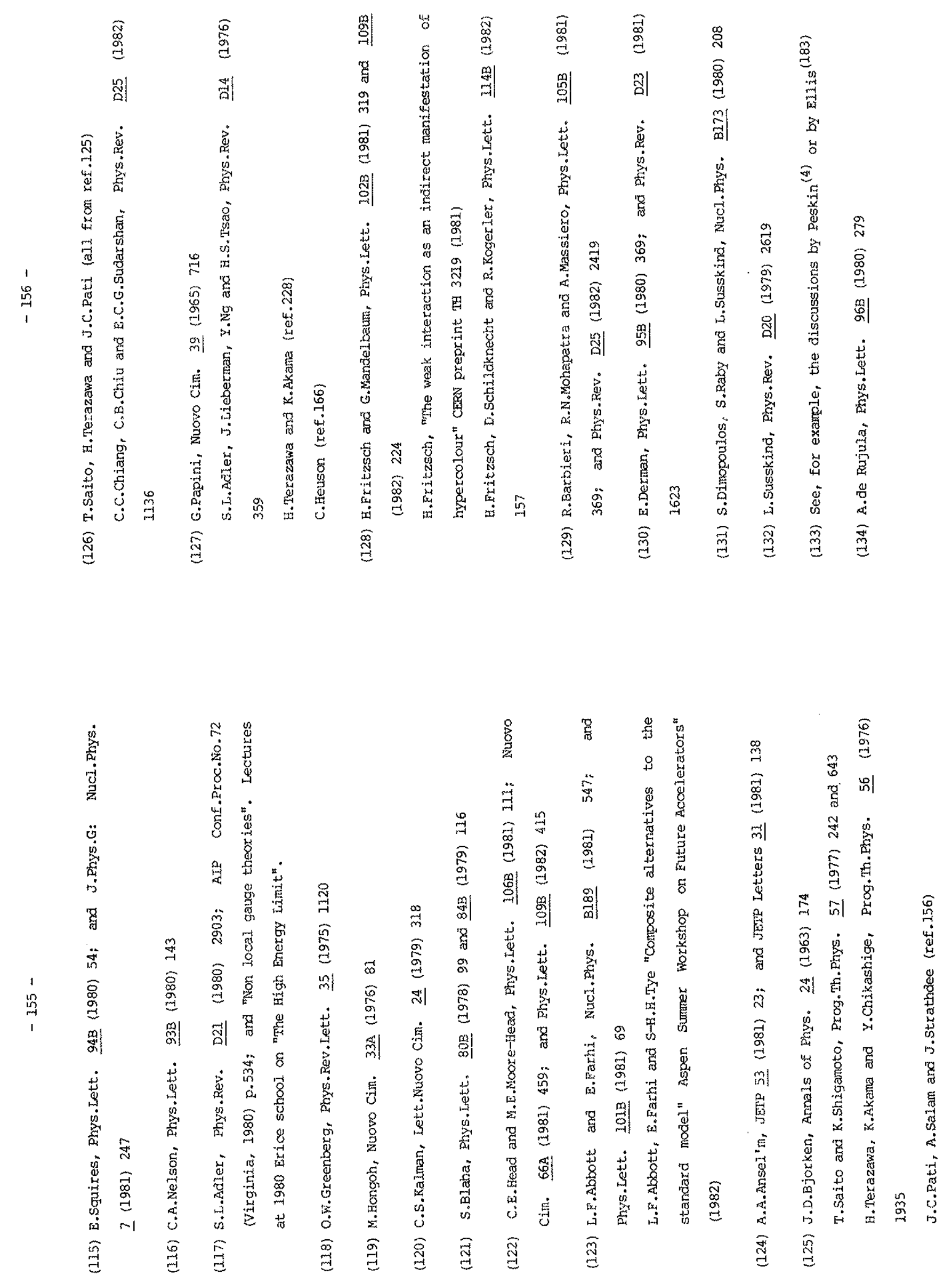

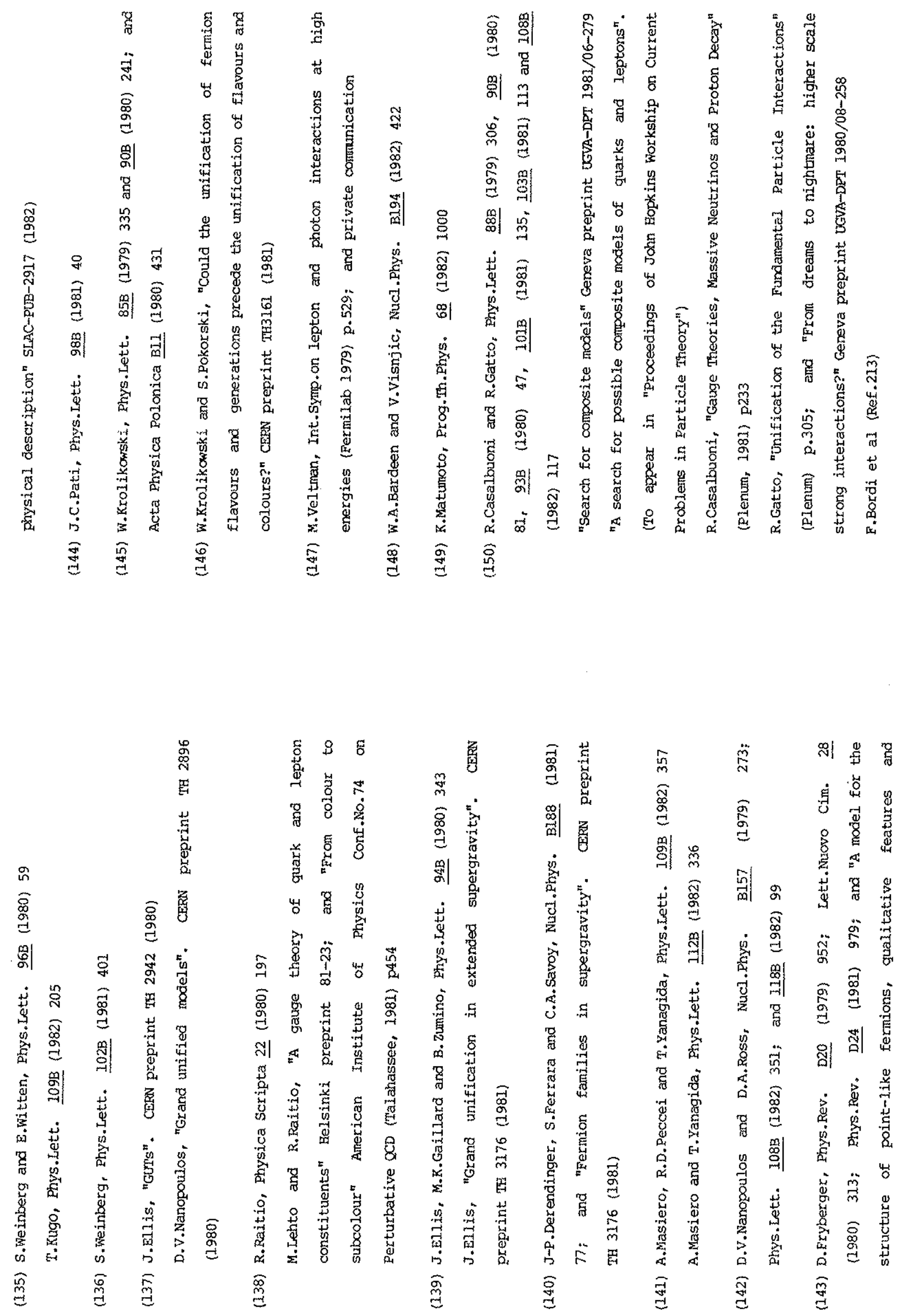

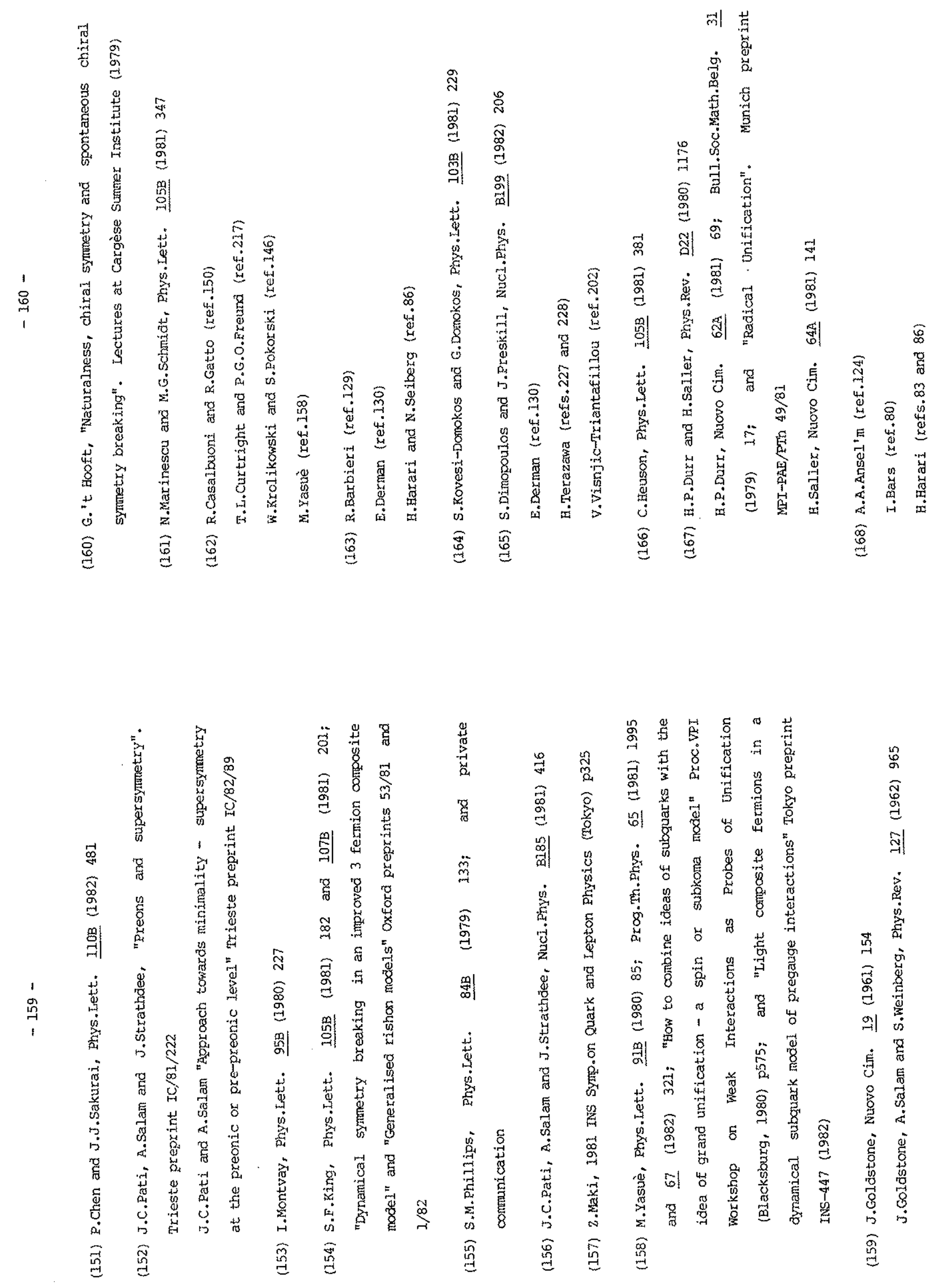

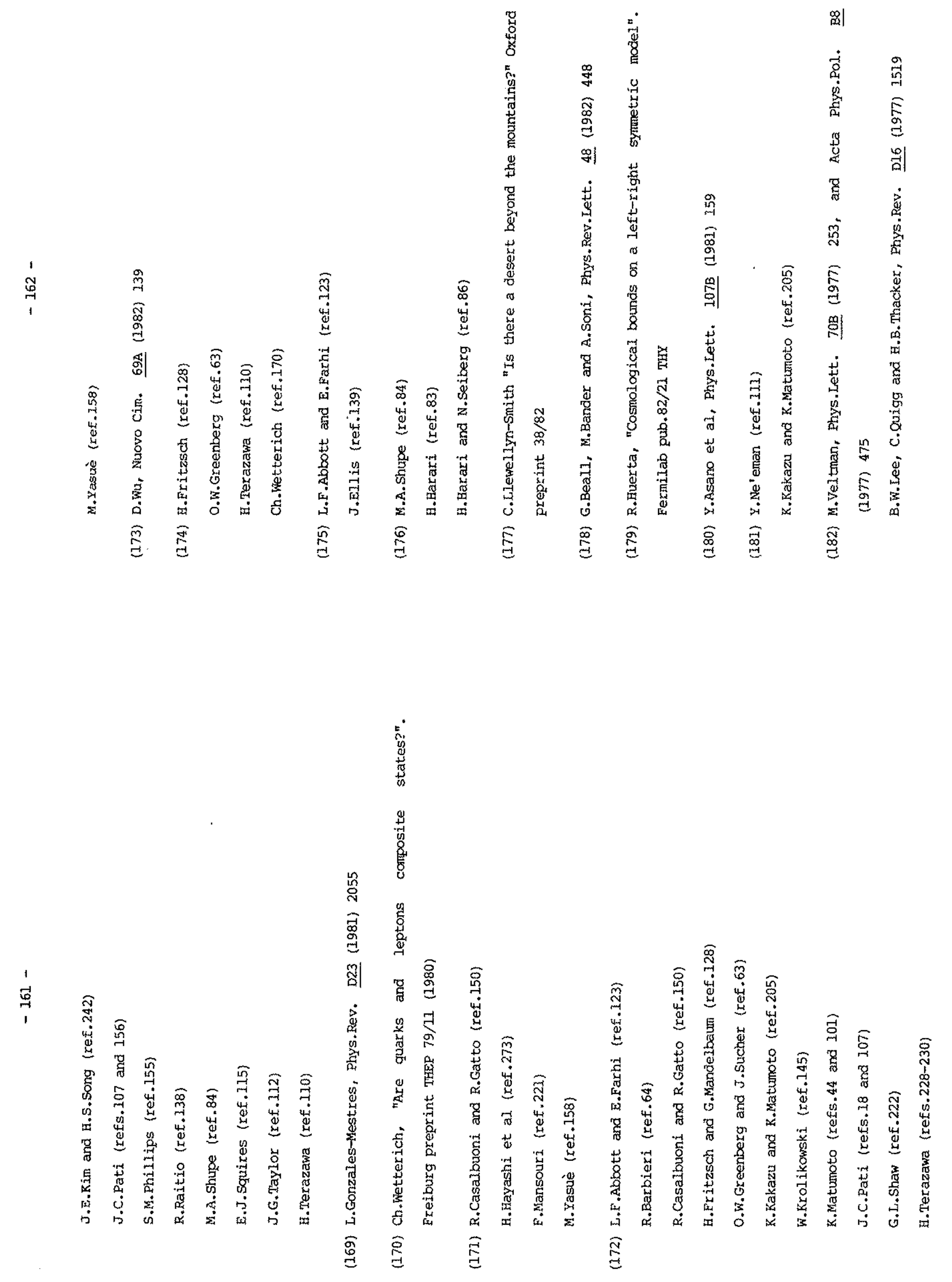
皇

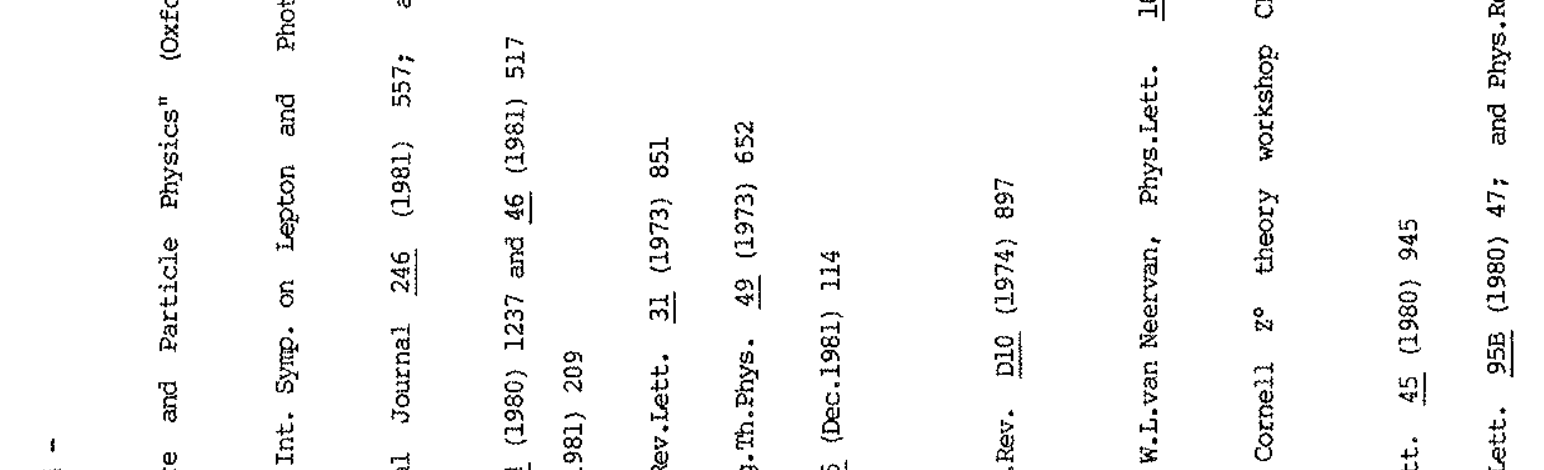

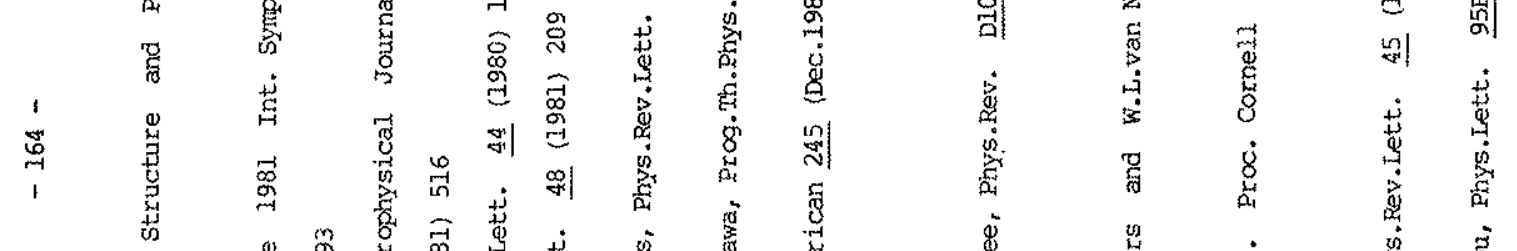

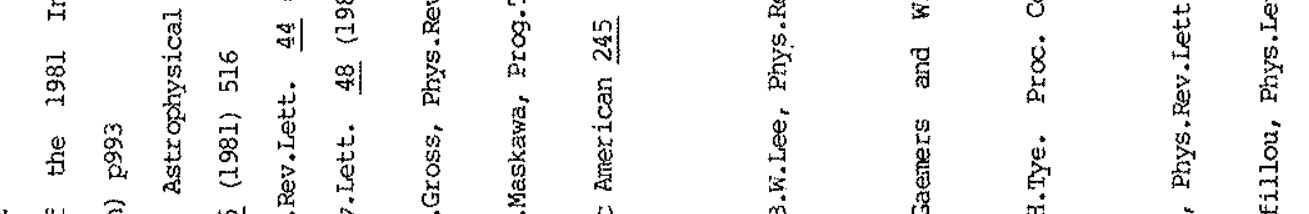

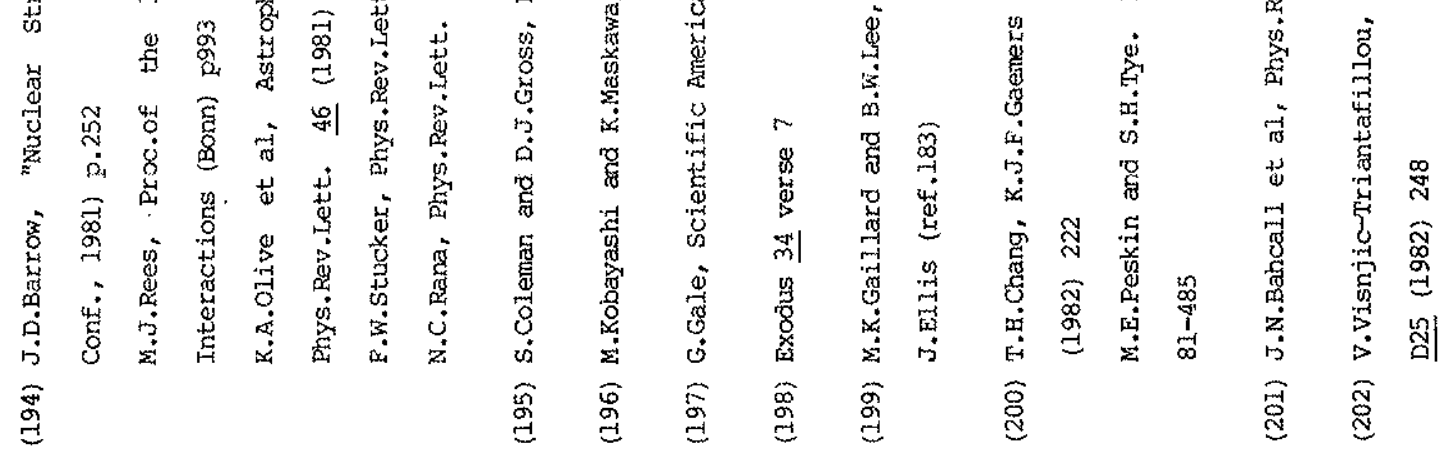

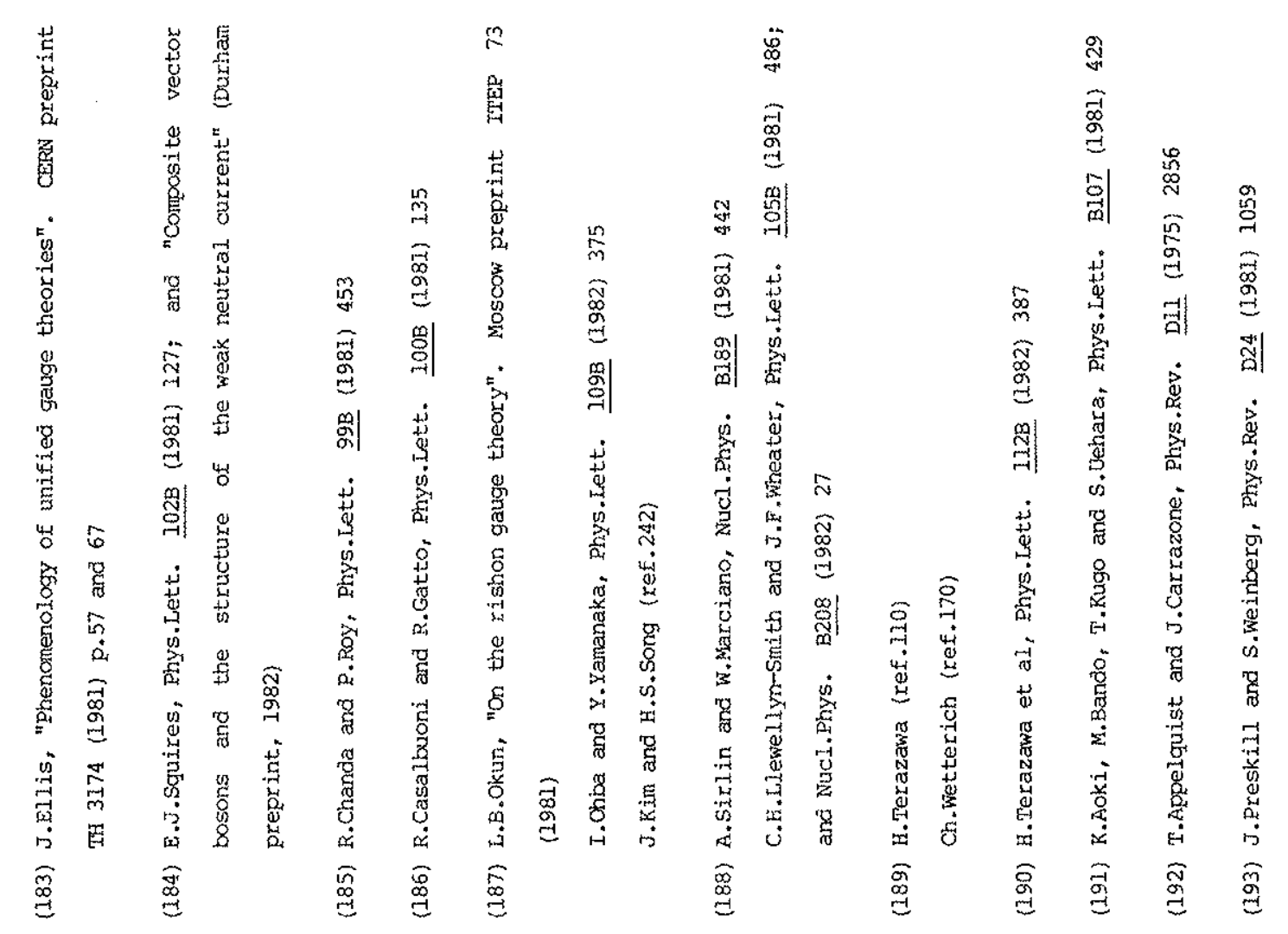




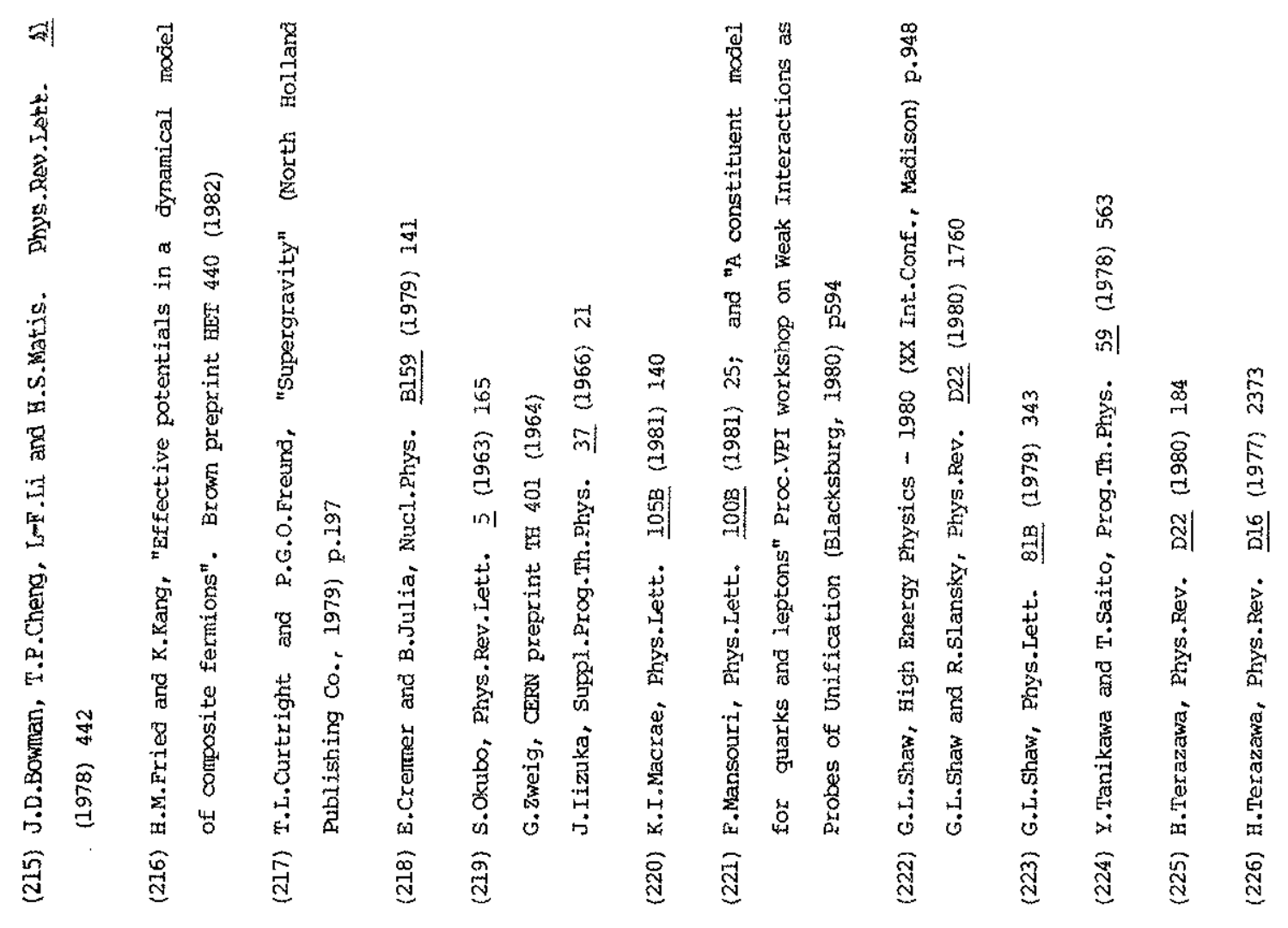

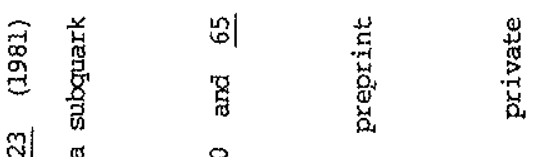

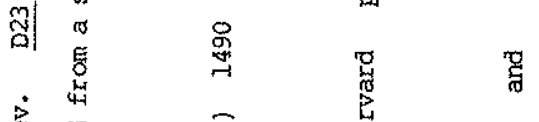

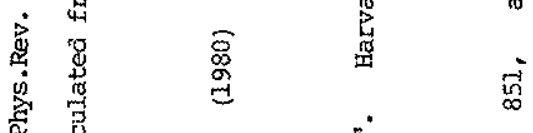

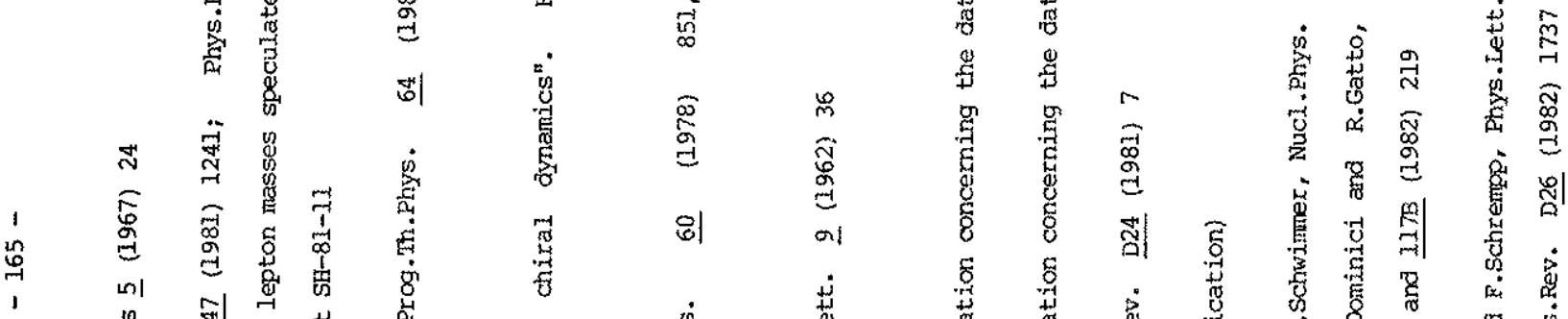

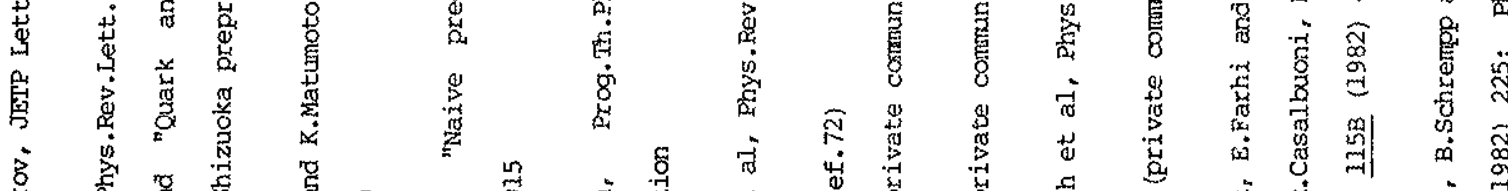

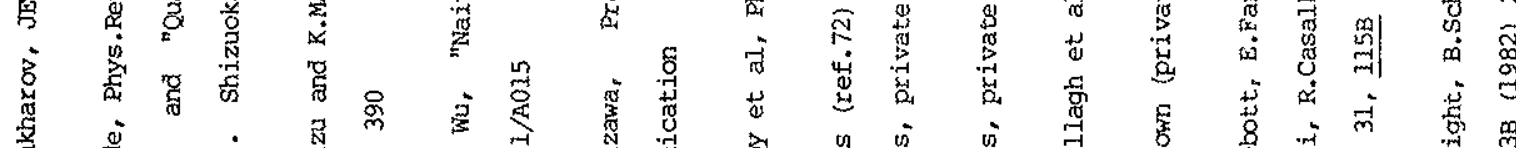

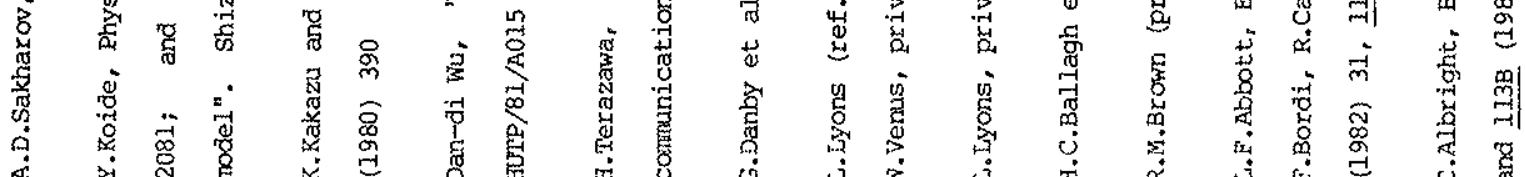

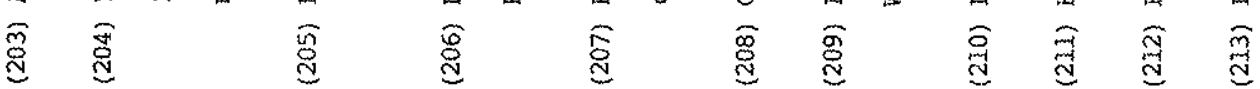

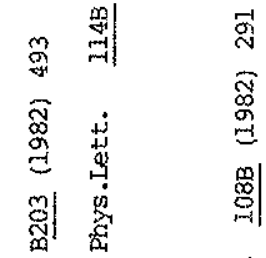

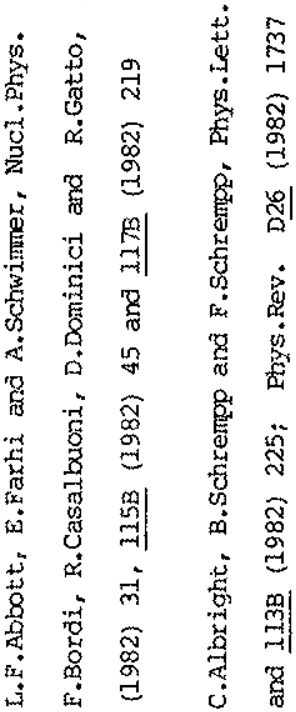

$\stackrel{\text { I }}{\text { 䎡 }}$ 


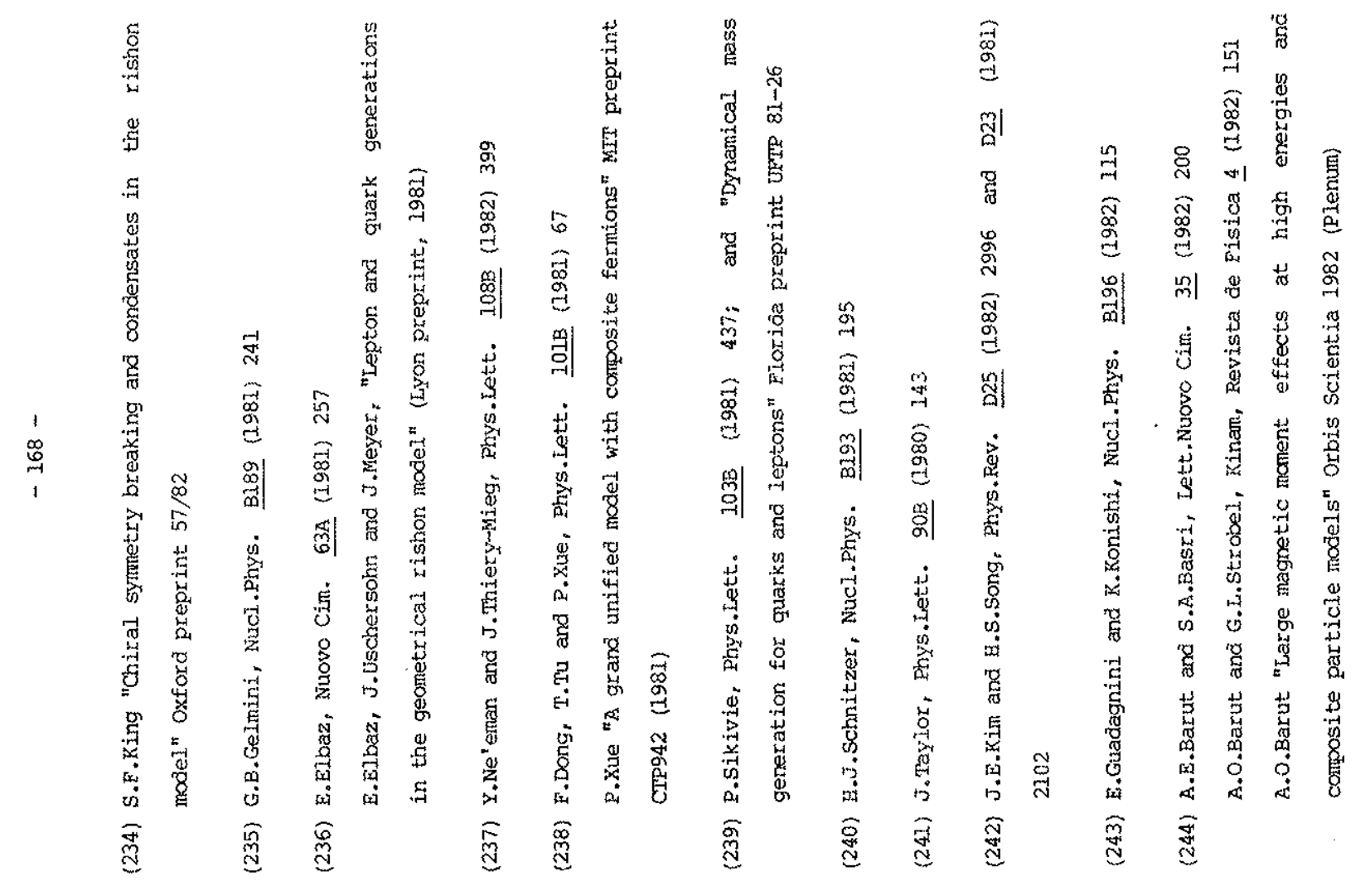

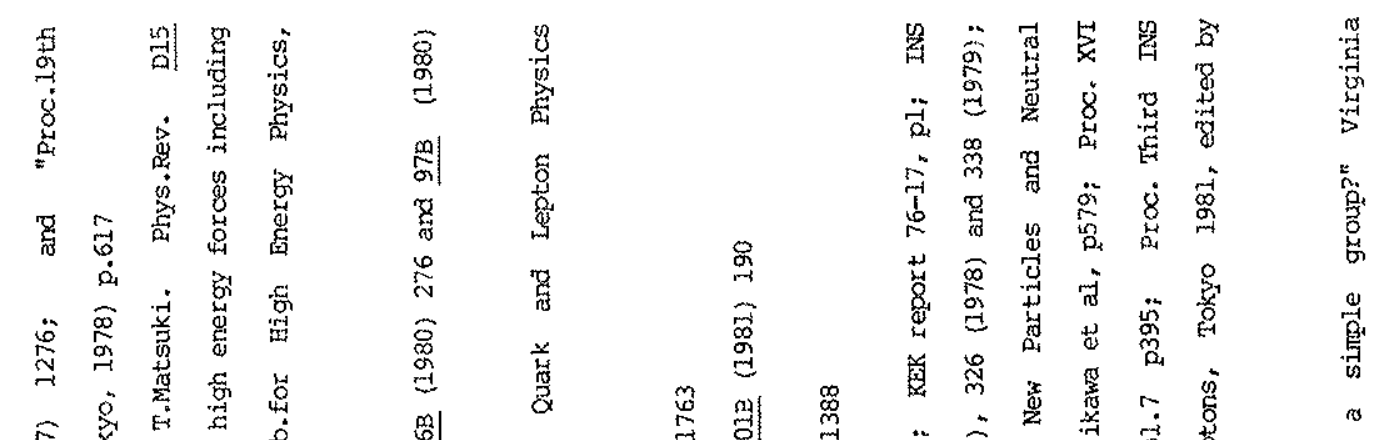

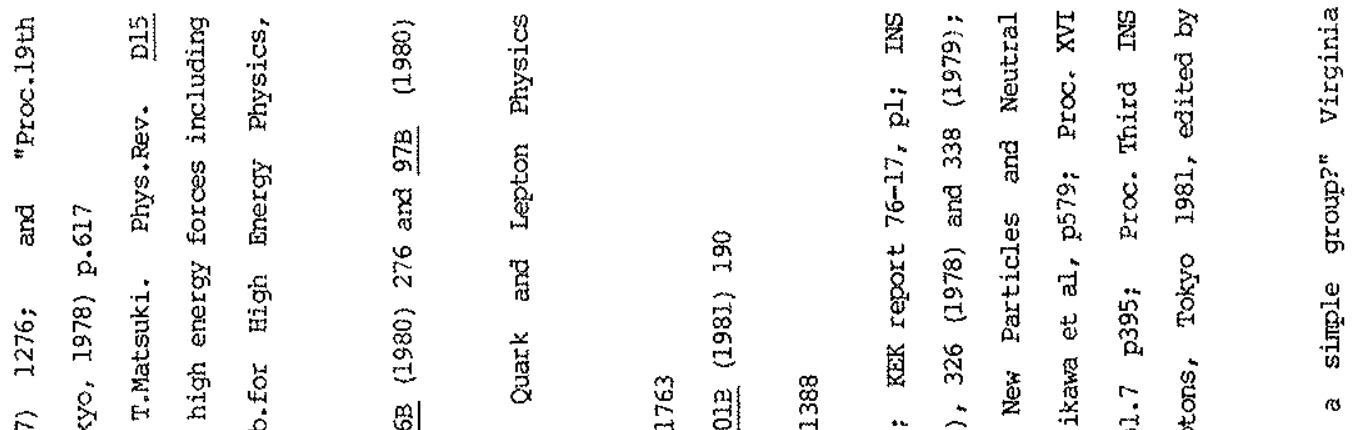

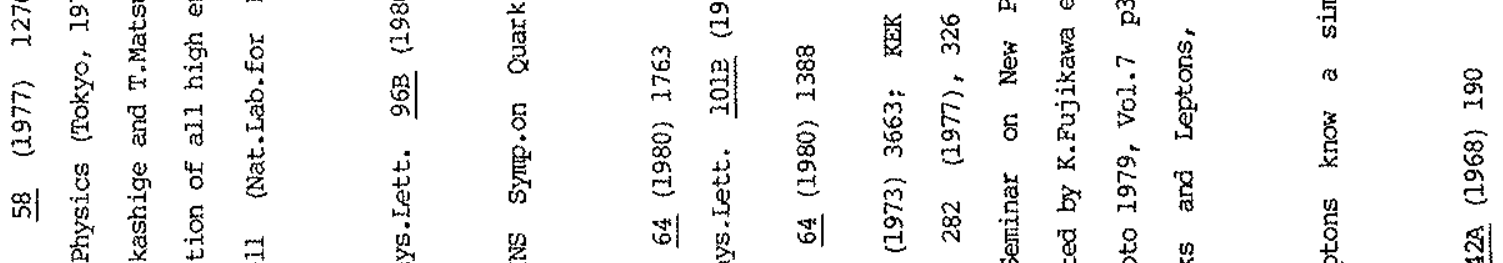

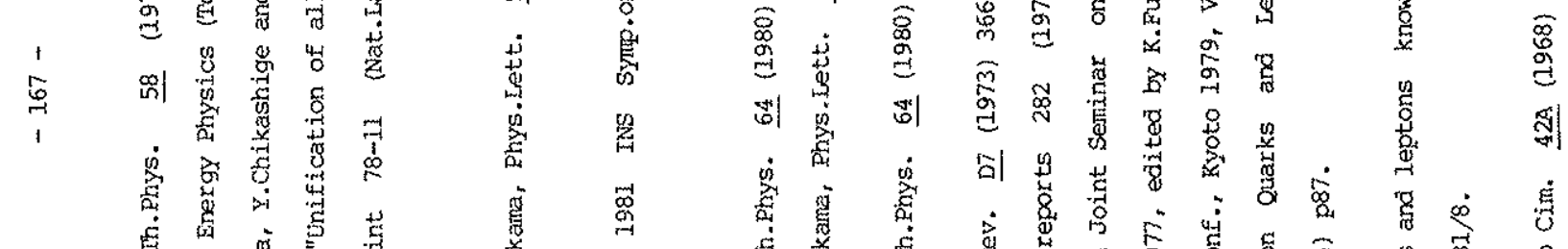

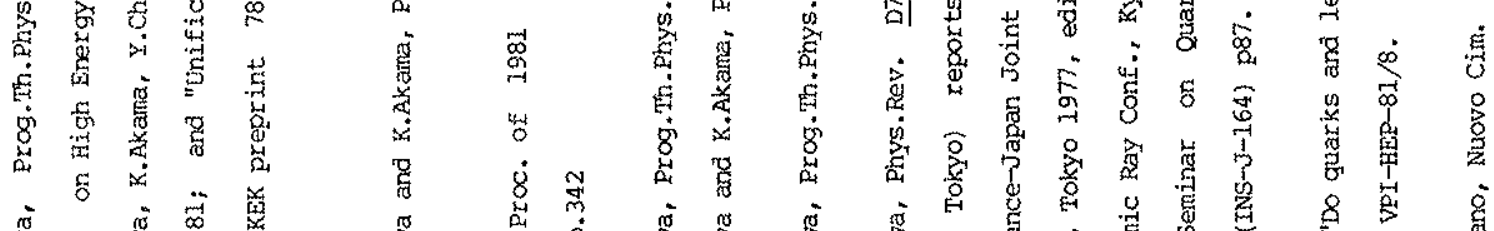

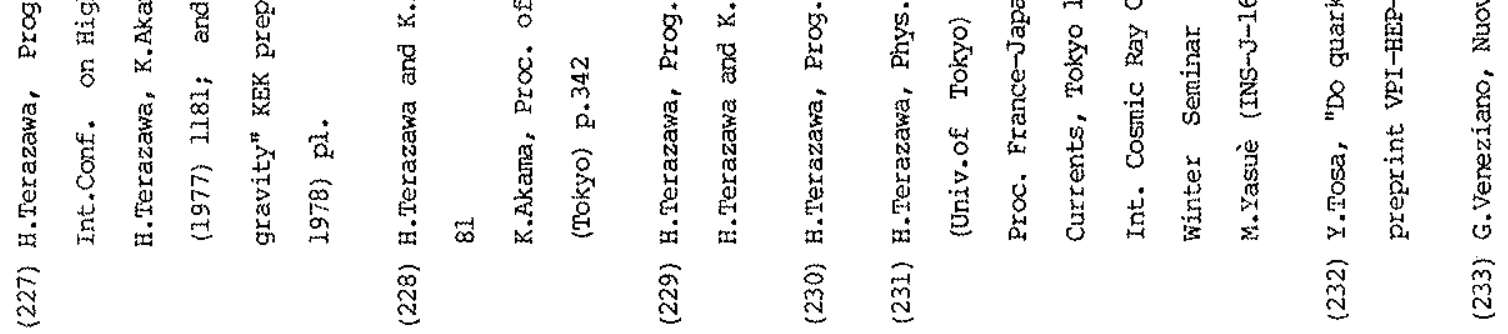




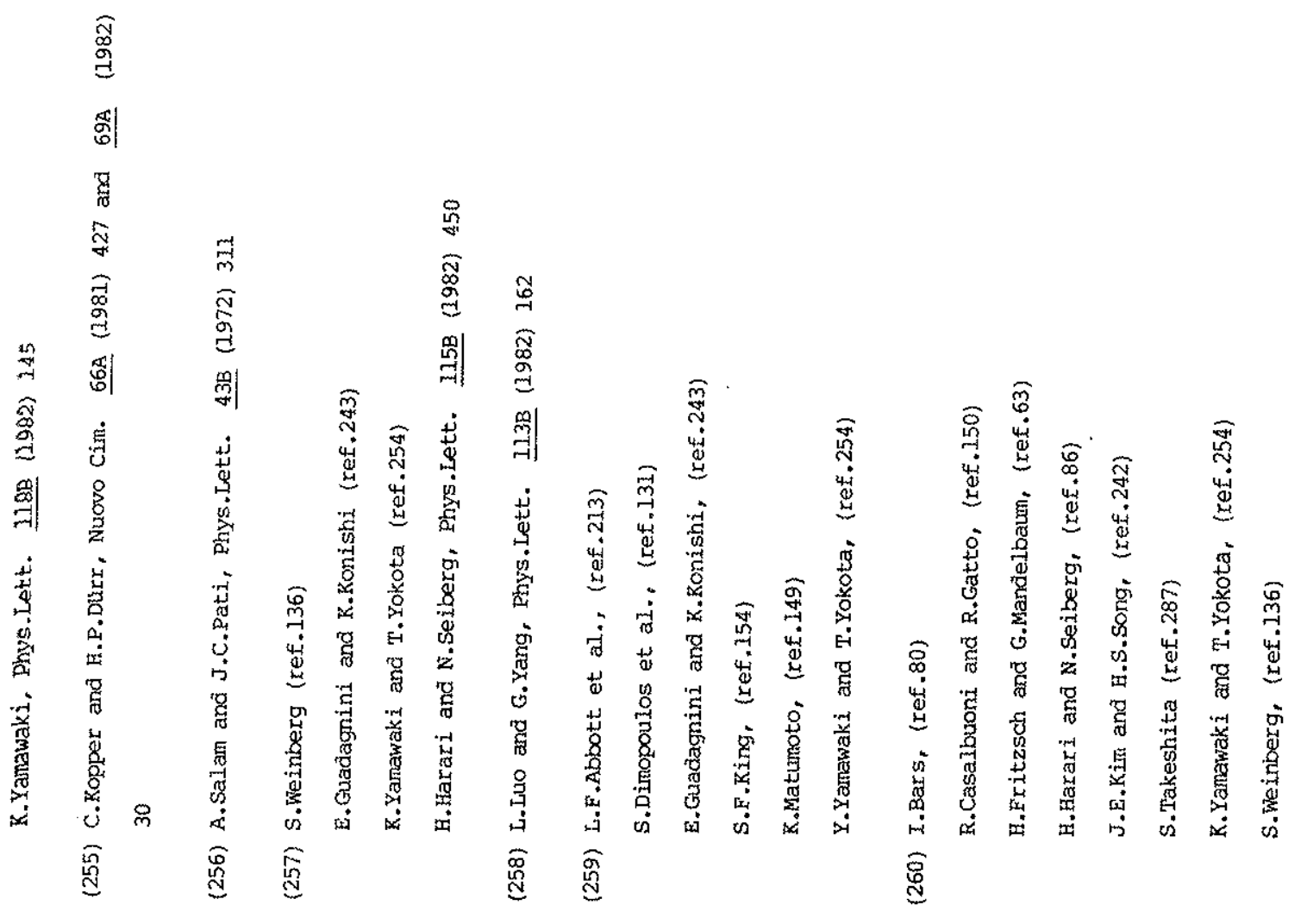

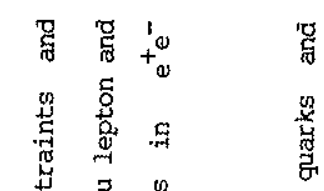

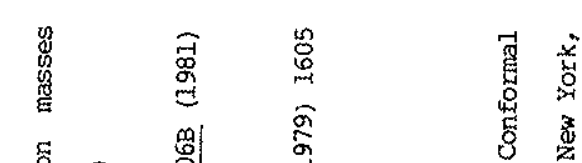

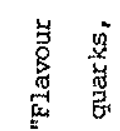

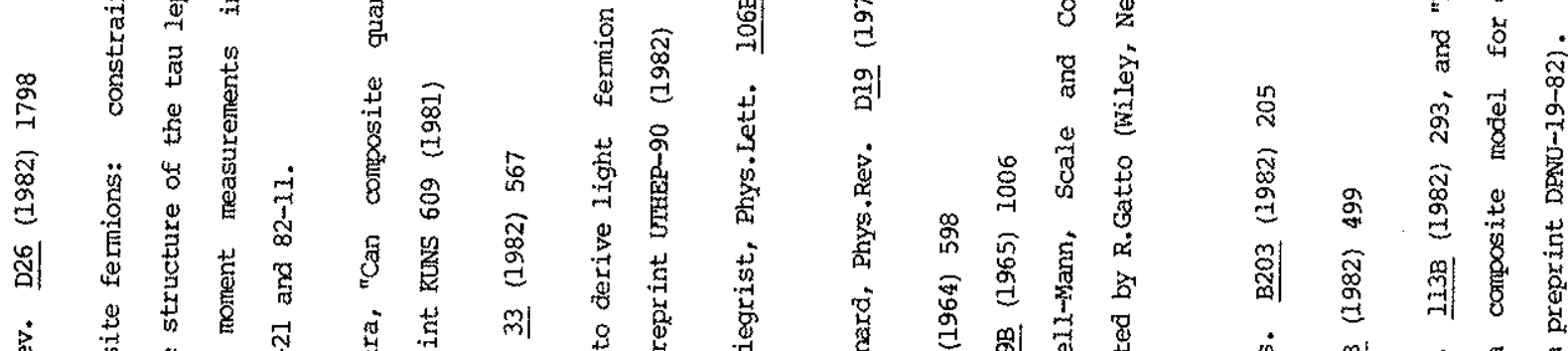

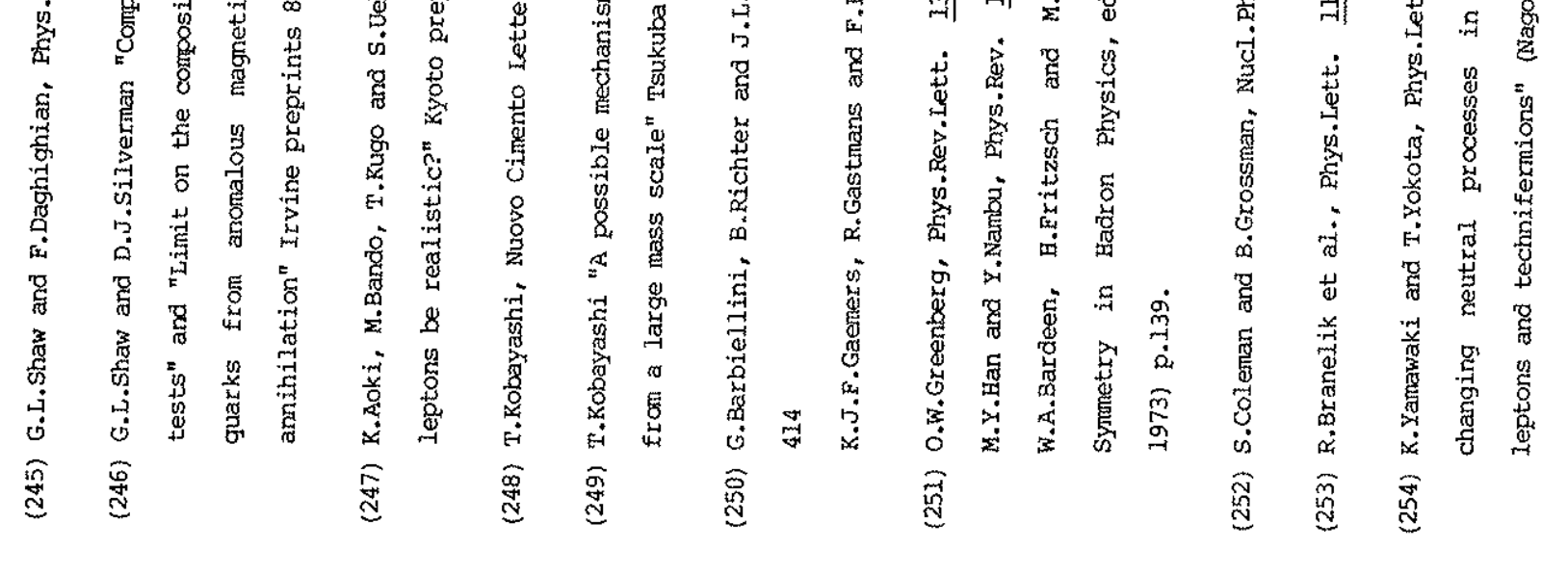




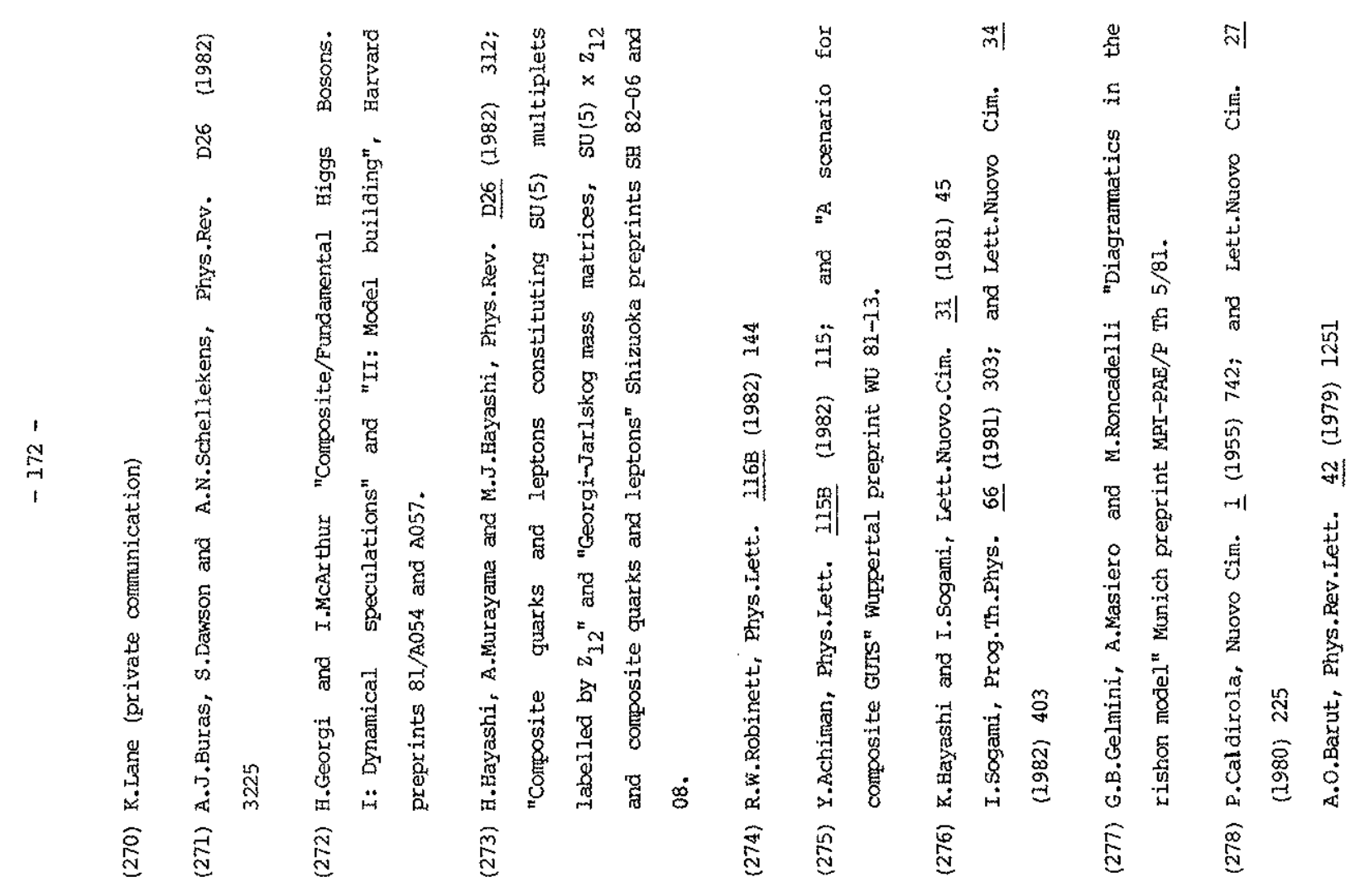

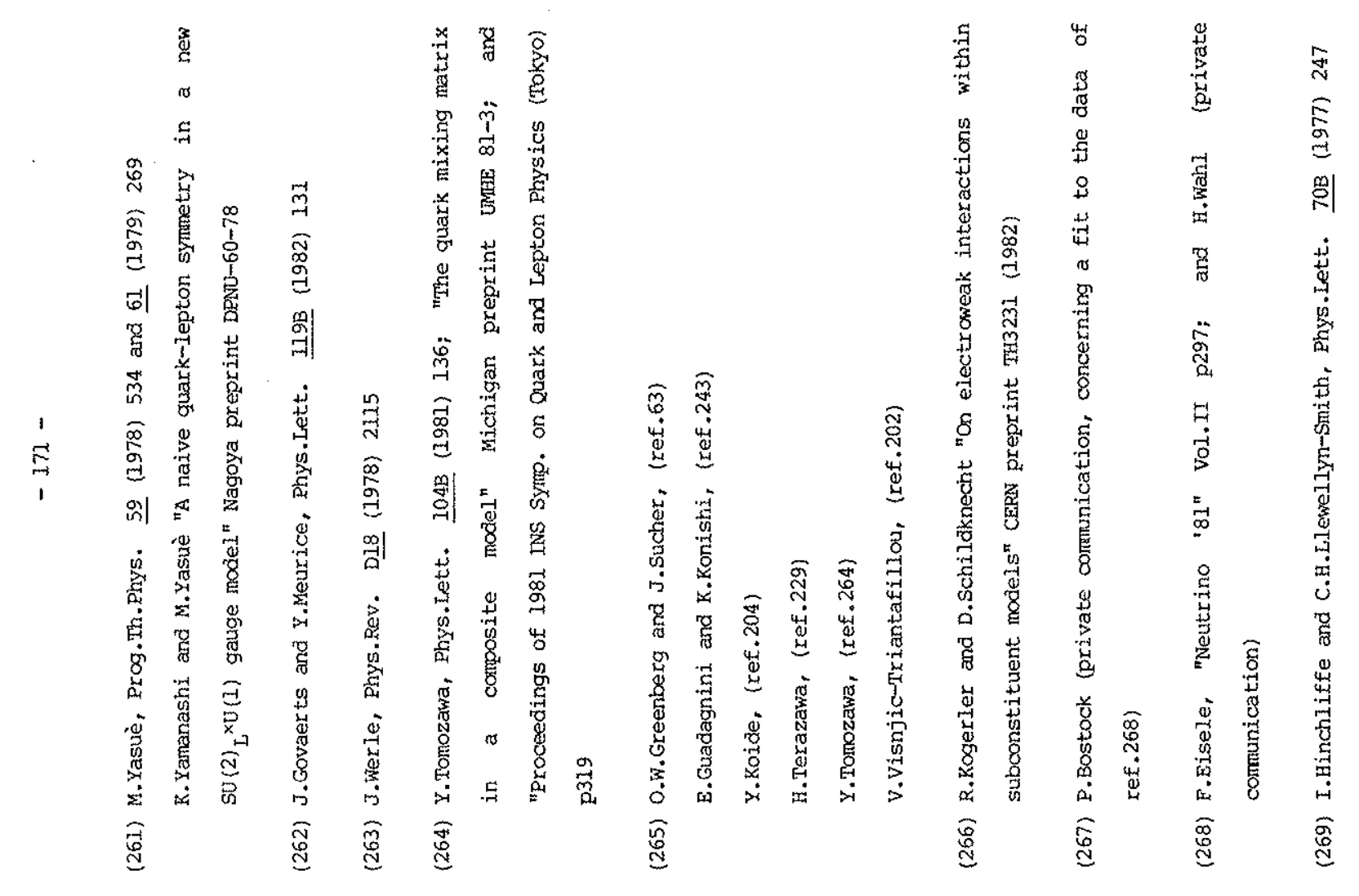




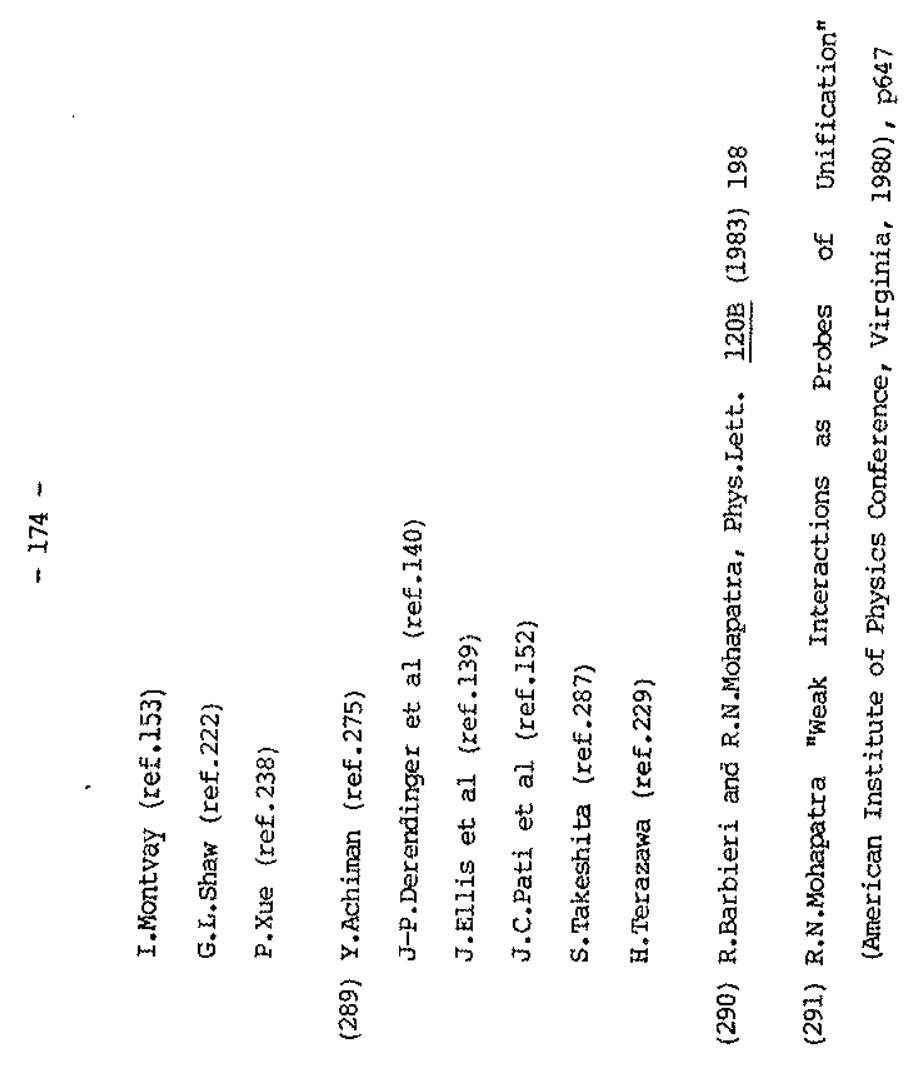

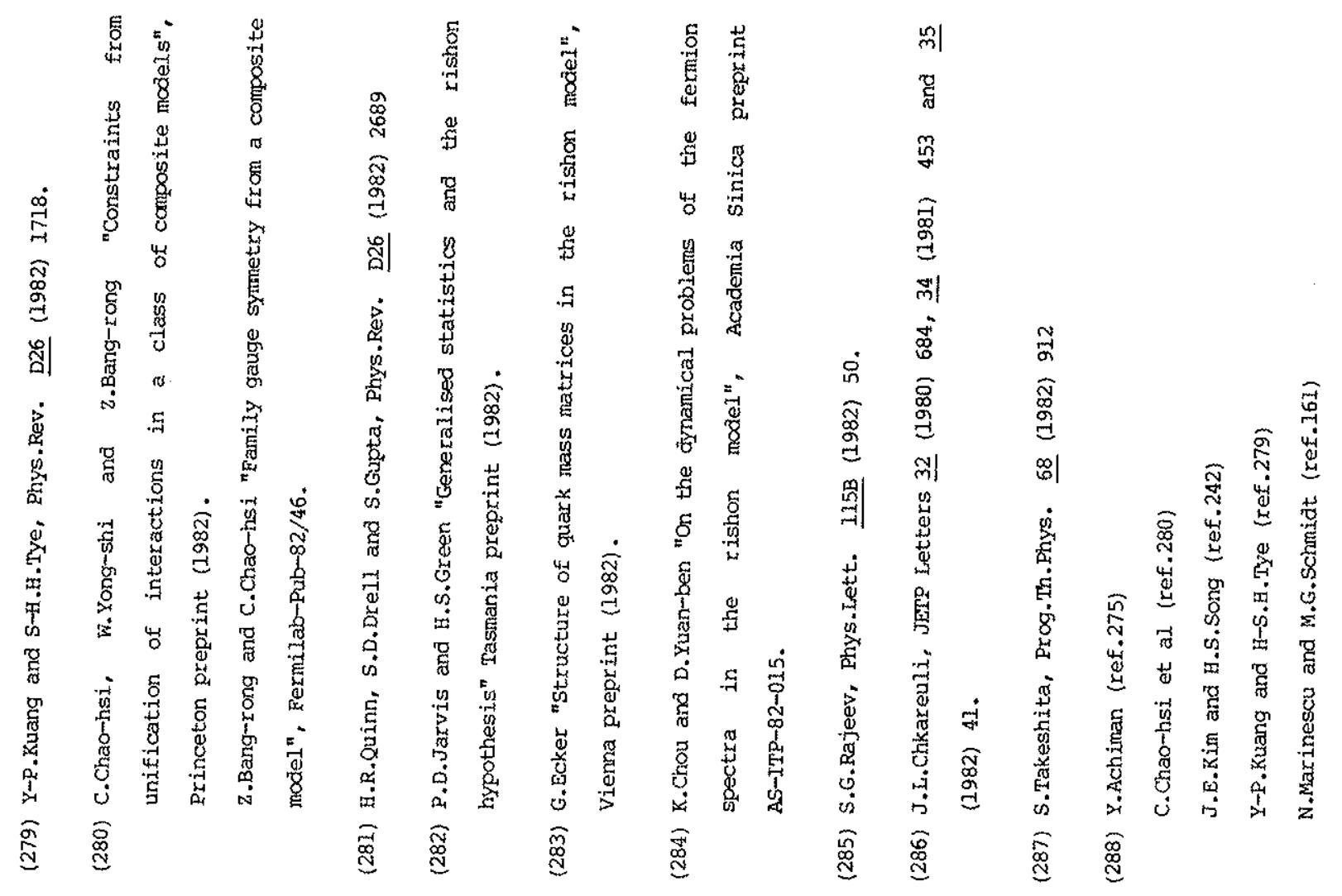



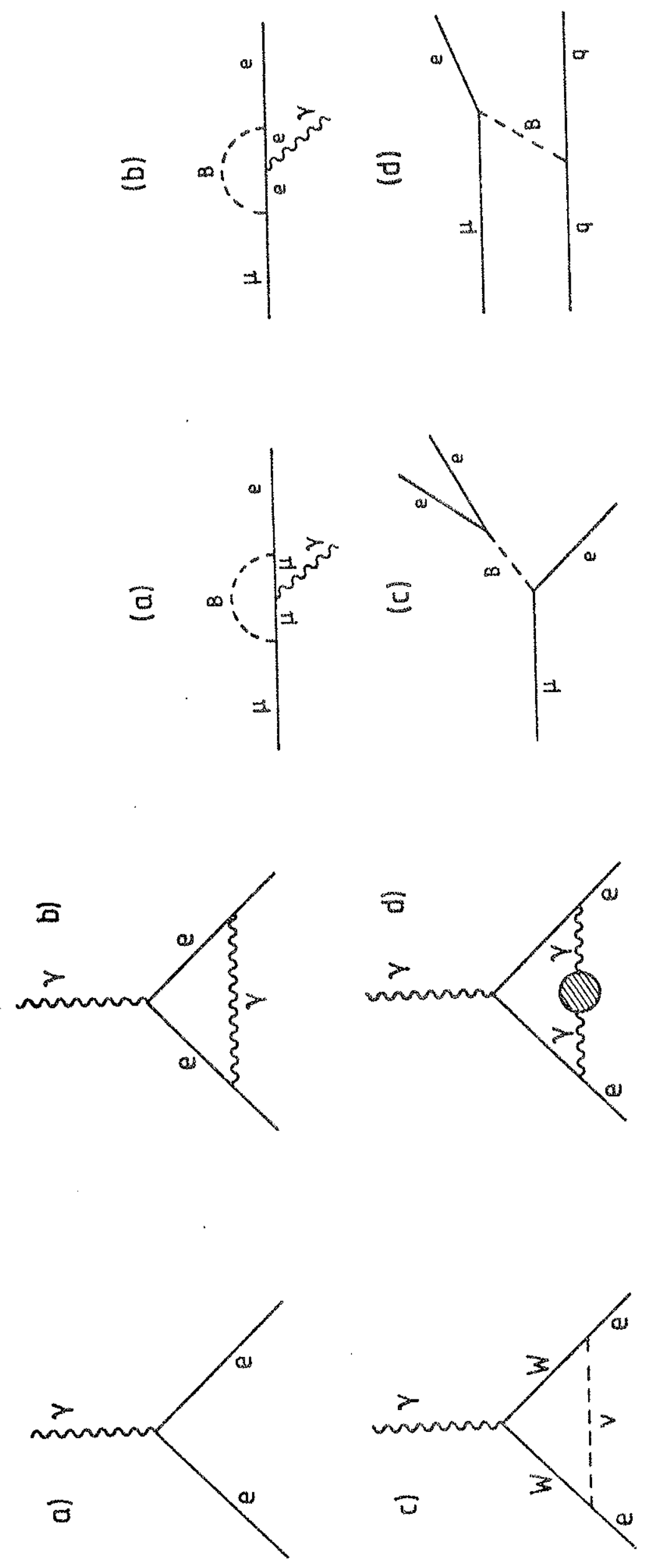

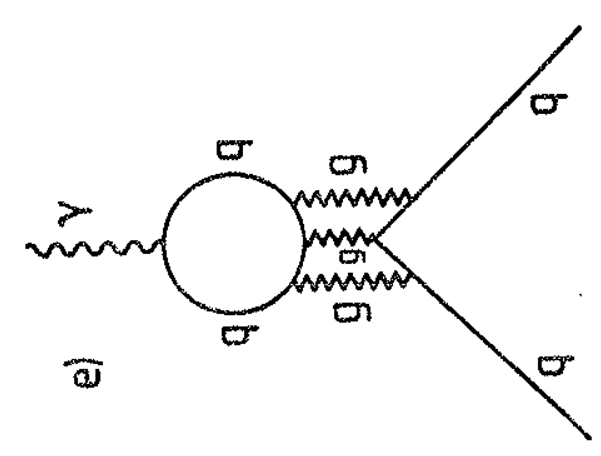



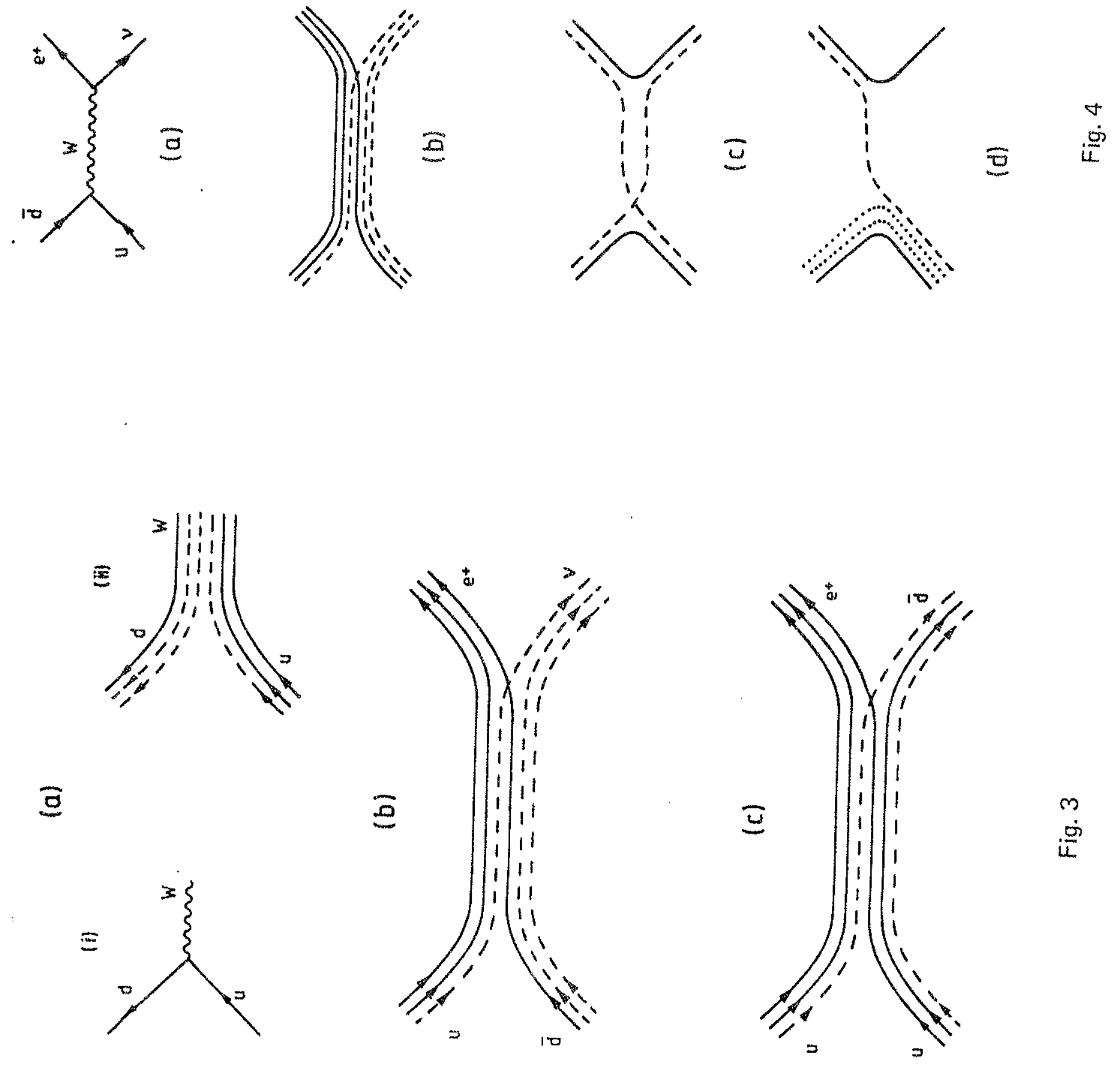


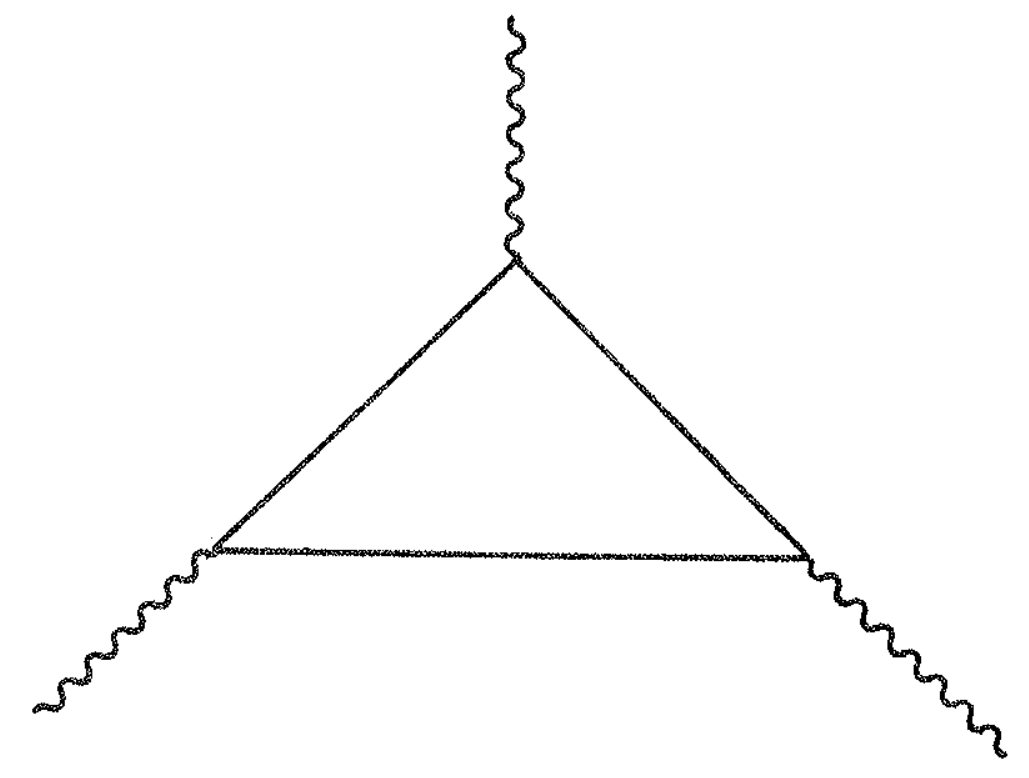

10

媳 